By

Rudhwan Issa Ahmed

\begin{abstract}
A thesis submitted to the Faculty of Graduate Studies and Postdoctoral Affairs
in partial fulfillment of the requirements for the degree of
\end{abstract}

MASTER OF APPLIED SCIENCE IN BIOMEDICAL ENGINEERING

(Ottawa - Carleton Institute for Biomedical Engineering (OCIBME))

Department of Systems and Computer Engineering

\author{
Carleton University \\ Ottawa, Canada, K15 5B6
}

December 2012

CCopyright 2012 Rudhwan Issa Ahmed 
Library and Archives

Canada

Published Heritage

Branch

395 Wellington Street

Ottawa ON K1A ON4

Canada
Bibliothèque et

Archives Canada

Direction du

Patrimoine de l'édition

395 , rue Wellington

Ottawa ON K1A ON4

Canada
Your file Votre référence

ISBN: 978-0-494-94248-2

Our file Notre référence

ISBN: $978-0-494-94248-2$
NOTICE:

The author has granted a nonexclusive license allowing Library and Archives Canada to reproduce, publish, archive, preserve, conserve, communicate to the public by telecommunication or on the Internet, loan, distrbute and sell theses worldwide, for commercial or noncommercial purposes, in microform, paper, electronic and/or any other formats.

The author retains copyright ownership and moral rights in this thesis. Neither the thesis nor substantial extracts from it may be printed or otherwise reproduced without the author's permission.
AVIS:

L'auteur a accordé une licence non exclusive permettant à la Bibliothèque et Archives Canada de reproduire, publier, archiver, sauvegarder, conserver, transmettre au public par télécommunication ou par l'Internet, prêter, distribuer et vendre des thèses partout dans le monde, à des fins commerciales ou autres, sur support microforme, papier, électronique et/ou autres formats.

L'auteur conserve la propriété du droit d'auteur et des droits moraux qui protege cette thèse. $\mathrm{Ni}$ la thèse ni des extraits substantiels de celle-ci ne doivent être imprimés ou autrement reproduits sans son autorisation.
In compliance with the Canadian Privacy Act some supporting forms may have been removed from this thesis.

While these forms may be included in the document page count, their removal does not represent any loss of content from the thesis.
Conformément à la loi canadienne sur la protection de la vie privée, quelques formulaires secondaires ont été enlevés de cette thèse.

Bien que ces formulaires aient inclus dans la pagination, il n'y aura aucun contenu manquant. 
The undersigned hereby recommend to the Faculty of Graduate Studies and Postdoctoral Affairs acceptance of the thesis

\title{
WIRELESS NETWORK SYSTEM BASED MULTI-NON-INVASIVE SENSORS FOR SMART HOME
}

\author{
Submitted by \\ Rudhwan Issa Ahmed \\ In partial fulfillment of the requirements for the degree of \\ MASTER OF APPLIED SCIENCE IN BIOMEDICAL ENGINEERING
}

Chair, Dr. Howard M. Schwartz, Department of Systems and Computer Engineering

Thesis Supervisor, Dr. Mohamed El-Tanany

Carleton University

December 2012 


\begin{abstract}
There are several techniques that have been implemented for smart homes usage; however, most of these techniques are limited to a few sensors. Many of these methods neither meet the needs of the user nor are cost-effective.
\end{abstract}

This thesis discusses the design, development, and implementation of a wireless network system, based on multi-non-invasive sensors for smart home environments. This system has the potential to be used as a means to accurately, and remotely, determine the activities of daily living by continuously monitoring relatively simple parameters that measure the interaction between users and their surrounding environment.

We designed and developed a prototype system to meet the specific needs of the elderly population. Unlike audio-video based health monitoring systems (which have associated problems such as the encroachment of privacy), the developed system's distinct features ensure privacy and are almost invisible to the occupants, thus increasing the acceptance levels of this system in household environments. The developed system not only achieved high levels of accuracy, but it is also portable, easy to use, cost-effective, and requires low data rates and less power compared to other wireless devices such as Wi-Fi, Bluetooth, wireless USB, Ultra wideband (UWB), or Infrared (IR) wireless.

Field testing of the prototype system was conducted at different locations inside and outside of the Minto Building (Centre for Advanced Studies in Engineering at Carleton University) as well as other locations, such as the washroom, kitchen, and living room of a prototype apartment. The main goal of the testing was to 
determine the range of the prototype system and the functionality of each sensor in different environments. After it was verified that the system operated well in all of the tested environments, data were then collected at the different locations for analysis and interpretation in order to identify the activities of daily living of an occupant. 


\section{ACKNOWLEDGMENTS}

It is with immense gratitude that I acknowledge the support and help of those who made this thesis both possible and an unforgettable experience for me.

First and foremost, I wish to thank God. I also have immense appreciation for my supervisor, Dr. Mohamed ElTanany. This work would have not been possible without the enthusiasm, inspiration, and, most of all, remarkable patience of Dr. El-Tanany. I would like to thank Dr. Rafik A. Goubran for giving me the opportunity to work on this research and for his expert guidance and support throughout this academic program.

Besides my supervisor and Dr. Rafik A. Goubran, I would like to thank the remaining members of my examining committee: Dr. Jiying Zhao from Ottawa University and Dr. Fei Richard Yu from Carleton University for their encouragement, insightful comments, and tough questions, which lead me to rethink my research in ways that I could not even have imagined.

Outside of my academic community, I would like to thank Anna Lee-Popham from Palindrome Translation and Editing (palindrome-fr-en.com) for her help in providing high-quality editing of the thesis.

I would also like to thank the Natural Sciences and Engineering Research Council of Canada (NSERC) and Carleton University for the graduate fellowship that made it financially possible for me to complete this thesis as well as for providing a stimulating and engaging environment in which to learn.

I would like to thank my friends Mamatjan Yasin, Wen-Lin Liu, and Laleh Adl, as well as all of those who supported me in any respect during the completion of the thesis. 
Lastly, I would like to thank my family for their supports, inspirations, and prayers. Thank you, may God bless you all. 


\section{TABLE OF CONTENTS}

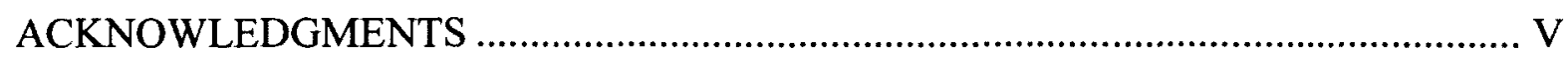

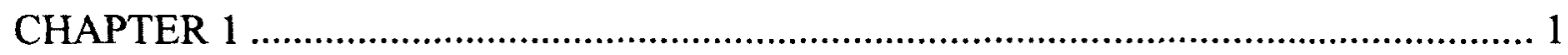

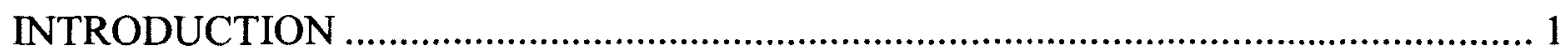

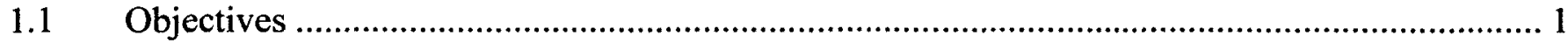

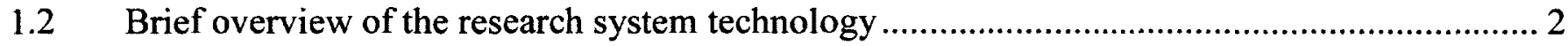

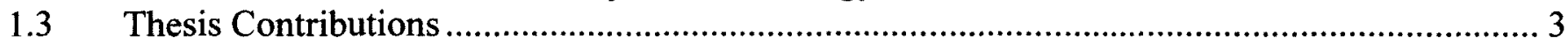

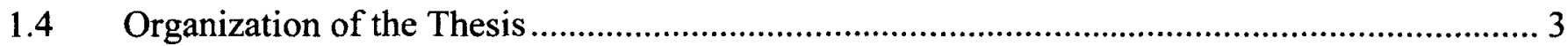

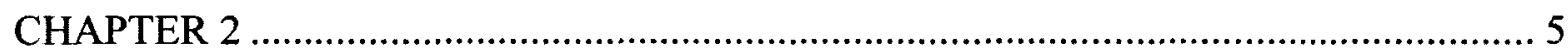

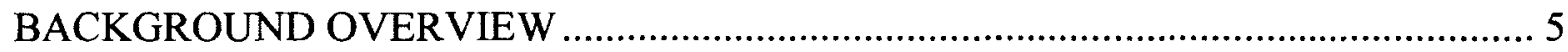

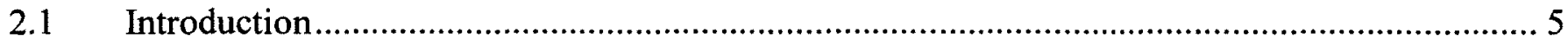

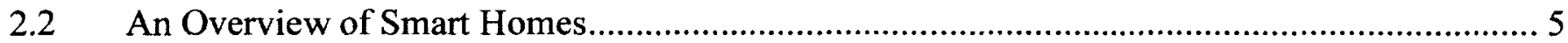

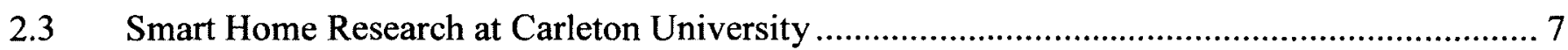

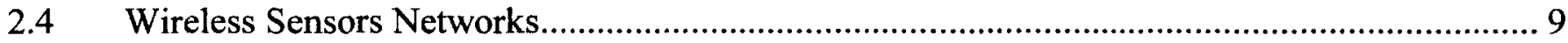

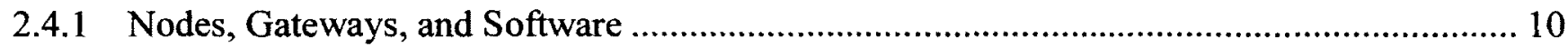

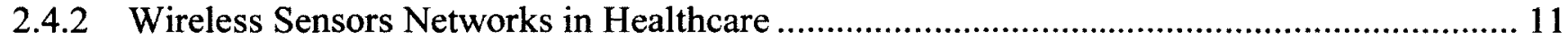

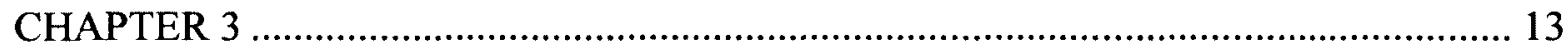

HARDWARE OVERVIEW AND SPECIFICATION .................................................. 13

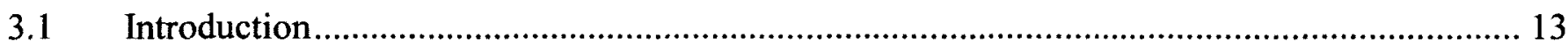

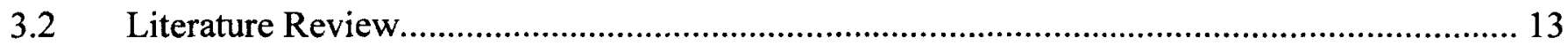

3.2.1 Wireless Sensor Networks for Home Health Care .............................................................. 14

3.2.2 XBee Wireless Sensor Network for Temperature Monitoring ............................................ 14

3.2.3 Design of Wireless Sensors Networking Node on ZigBee .................................................... 15

3.2.4 Development of a PIC-Based Wireless Sensor ................................................................ 15

3.2.5 Performance Study on ZigBee-Based Wireless Personal Area Networks for Health

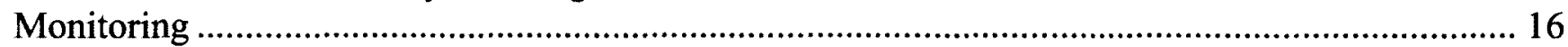

3.2.6 Temperature Data Logger Using IEEE 802.15.4/ZigBee Protocol ................................... 16

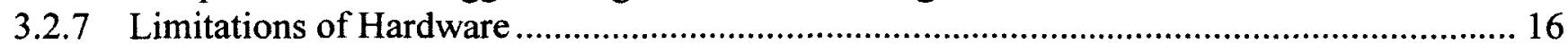

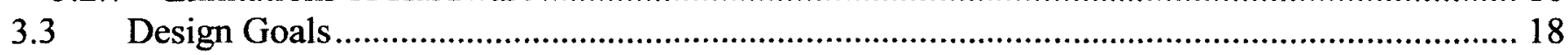

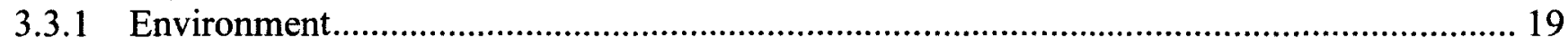

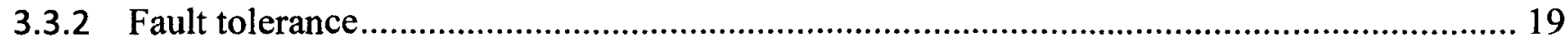

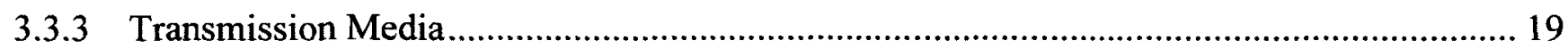

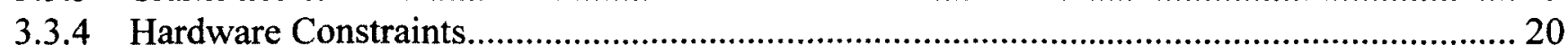




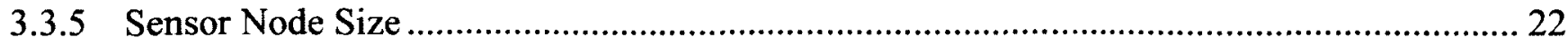

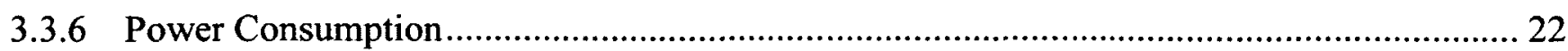

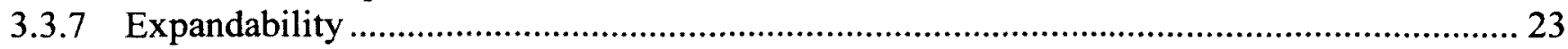

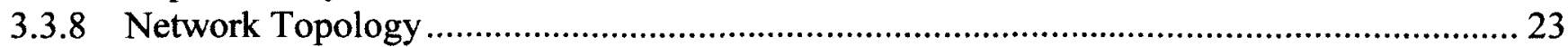

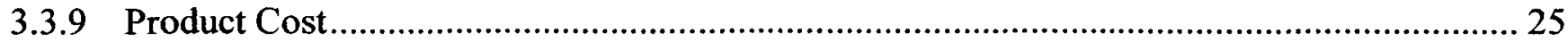

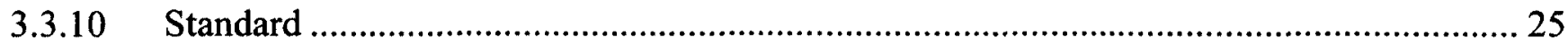

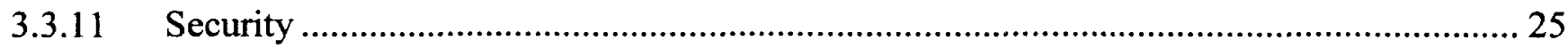

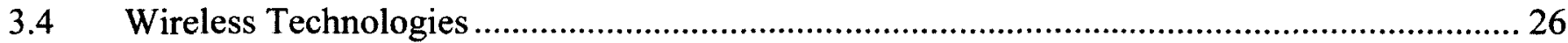

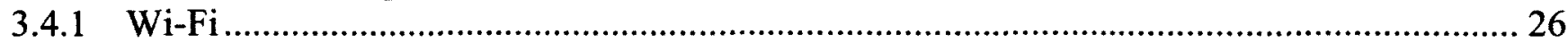

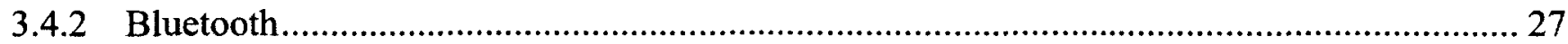

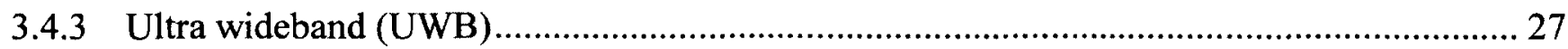

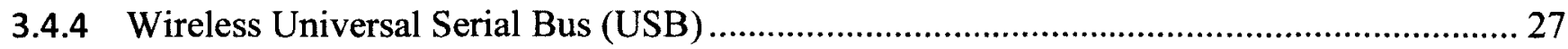

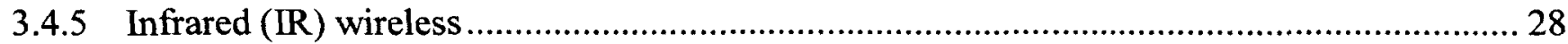

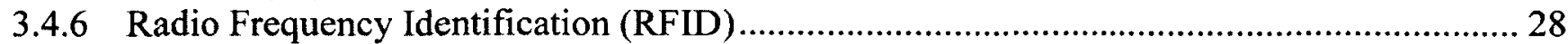

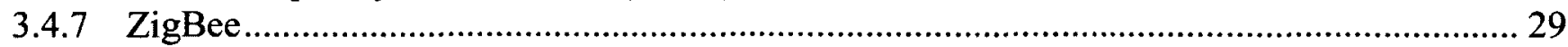

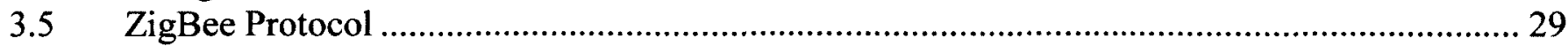

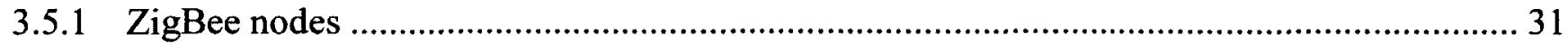

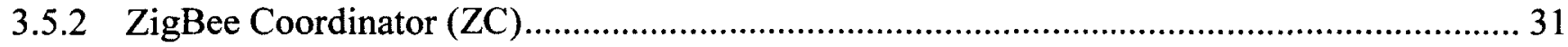

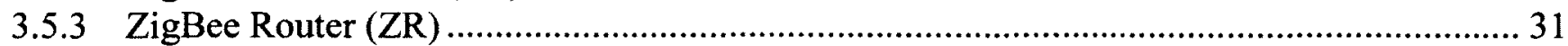

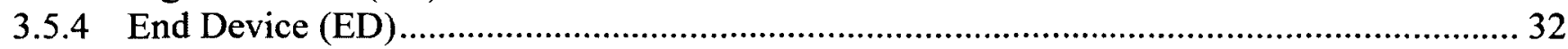

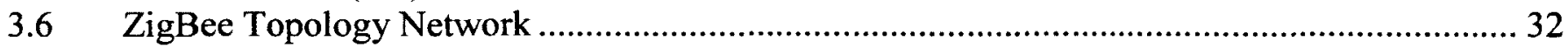

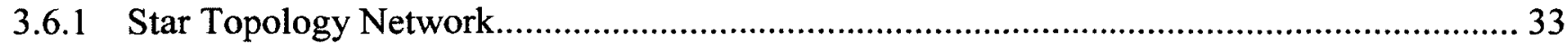

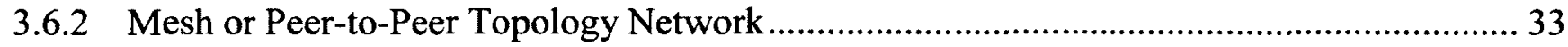

3.6.3 Cluster Tree Topology Network

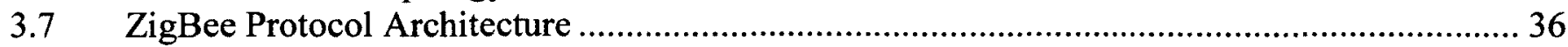

3.7.1 IEEE 802.15.4

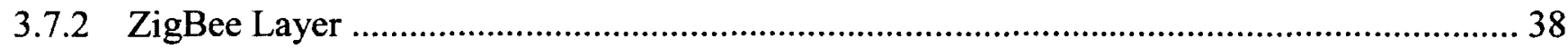

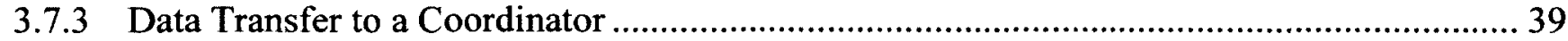

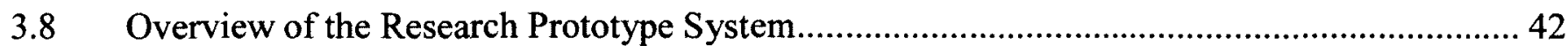

3.9 Components of the Research Prototype System ................................................................. 44

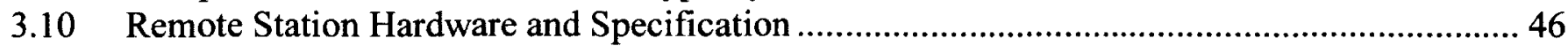

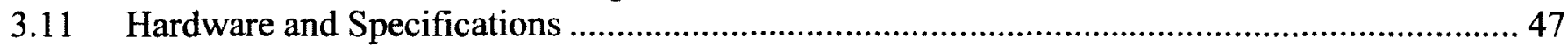

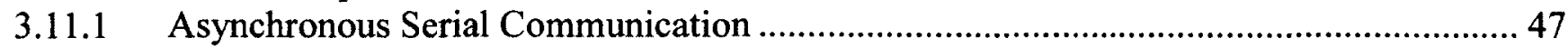

3.11.2 Universal Asynchronous Receiver and Transmitter (USART) Protocol .......................... 48

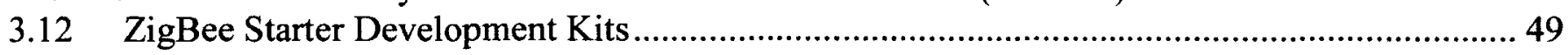

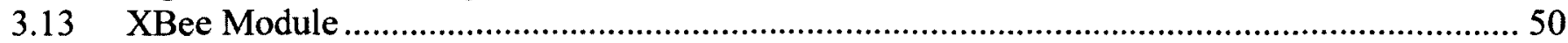

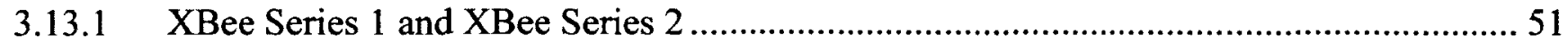

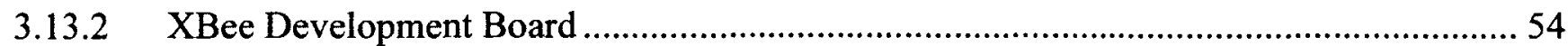

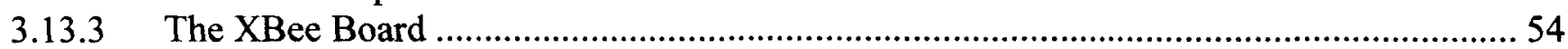




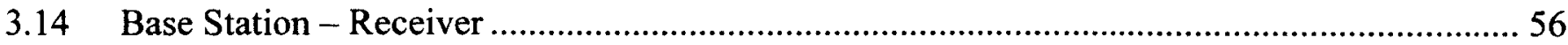

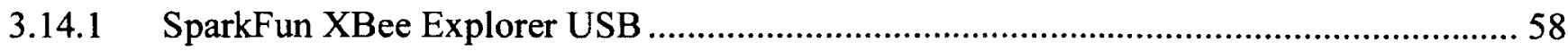

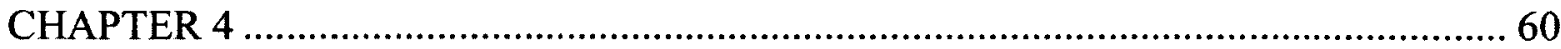

DESCRIPTION OF THE HARDWARE AND SOFTWARE …….................................6 60

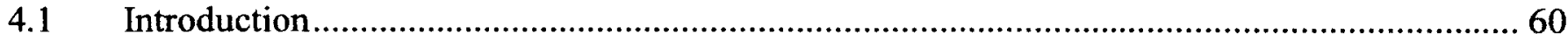

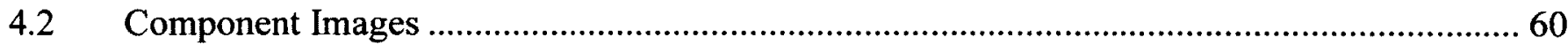

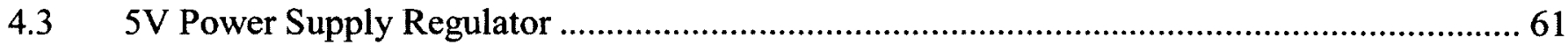

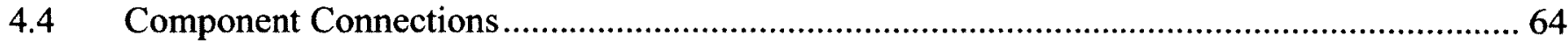

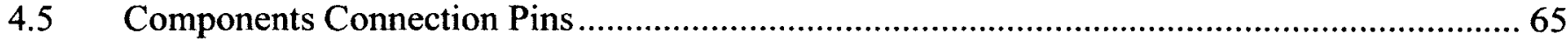

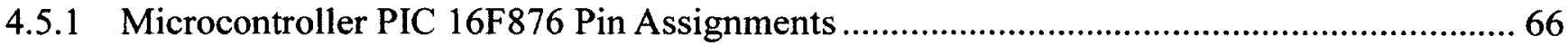

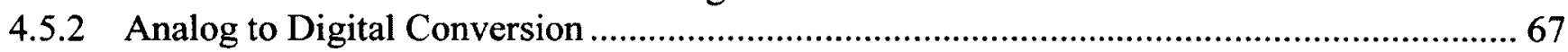

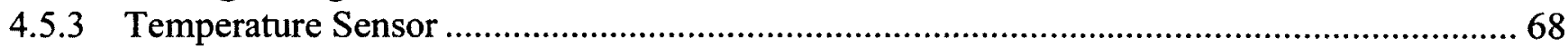

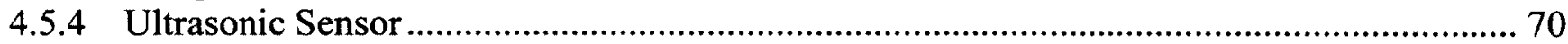

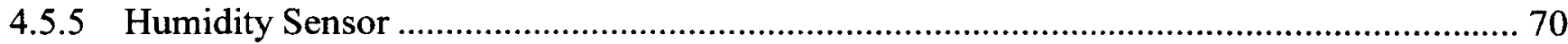

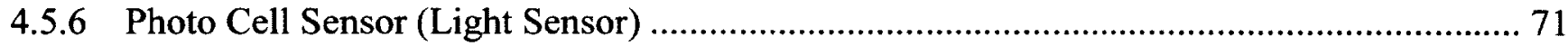

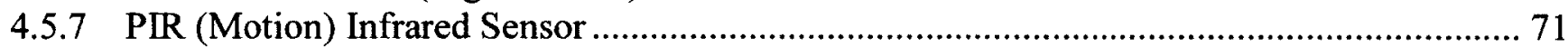

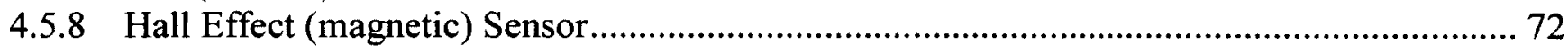

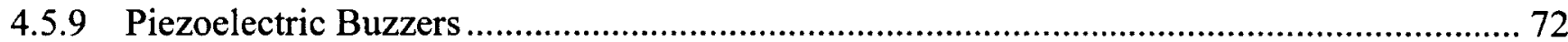

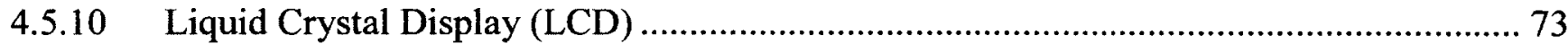

4.5.11 XBee Module Communication Protocol......................................................................... 73

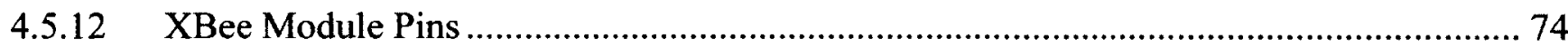

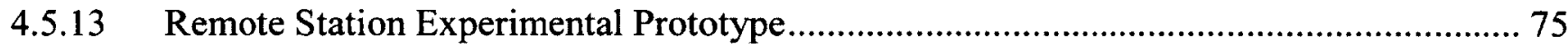

4.5.14 The main experimental prototype components......................................................... 76

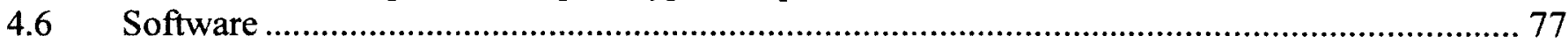

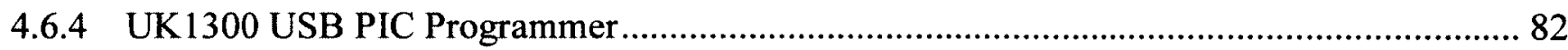

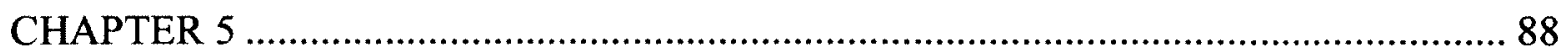

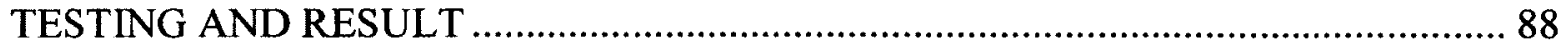

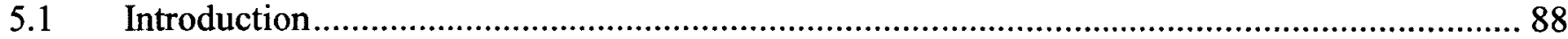

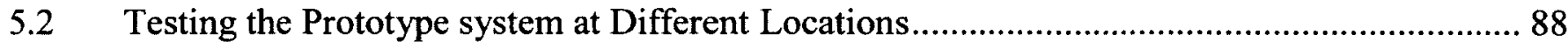

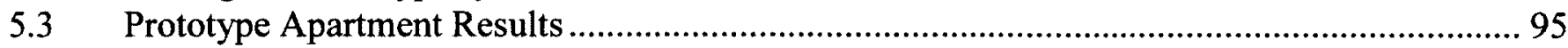

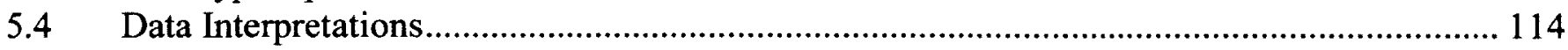

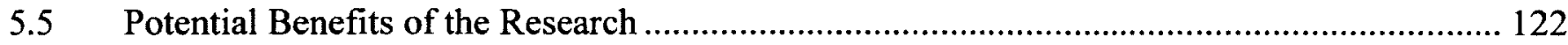

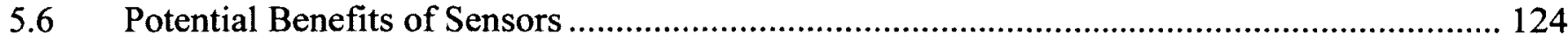

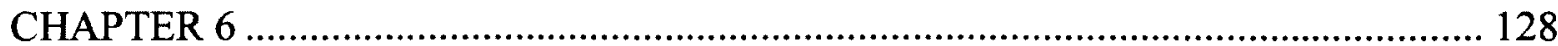




\section{CONCLUSION, LIMITATIONS, AND SUGGESTIONS FOR FUTURE RESEARCH128}

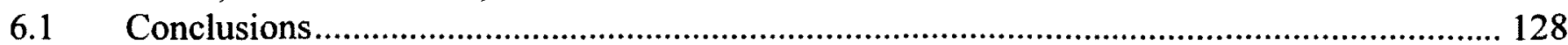

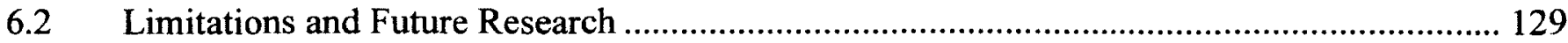

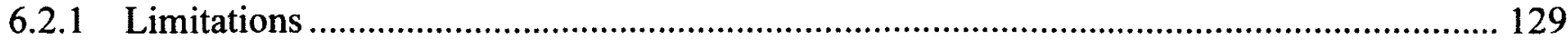

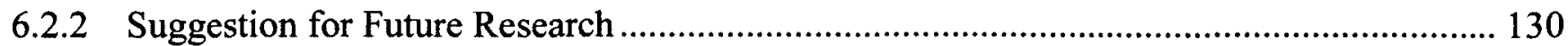

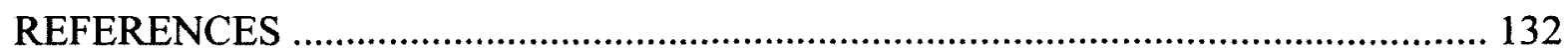

APPENDIX

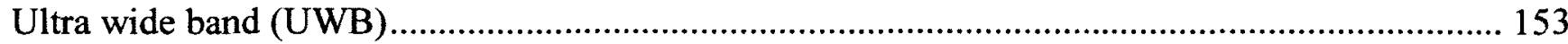

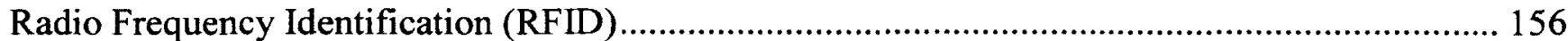

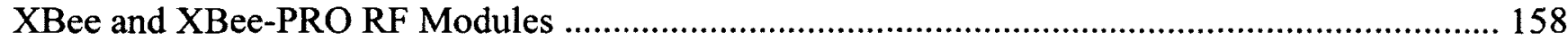

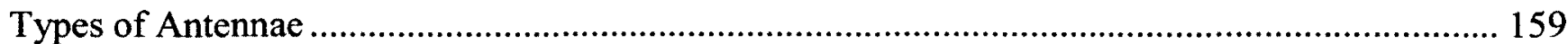

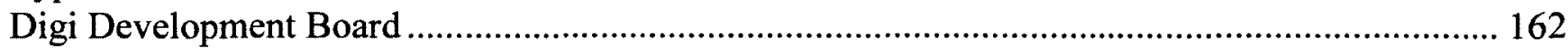

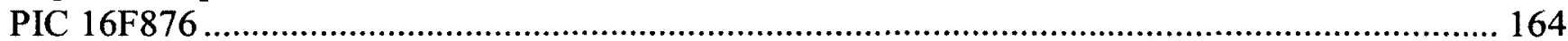

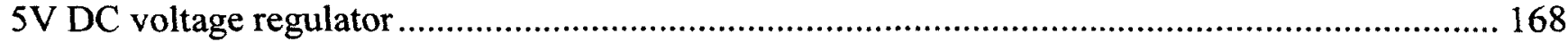

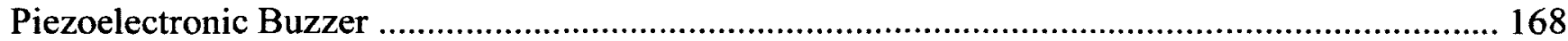

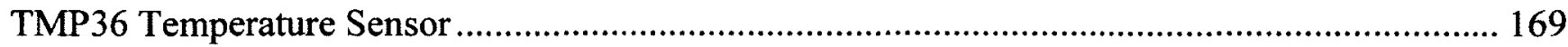

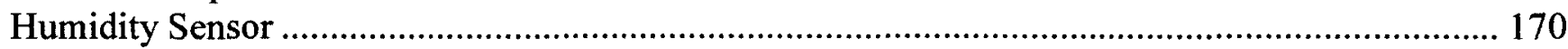

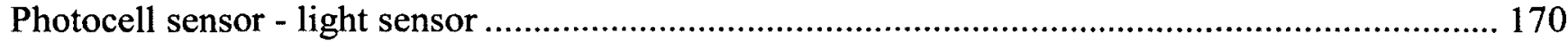

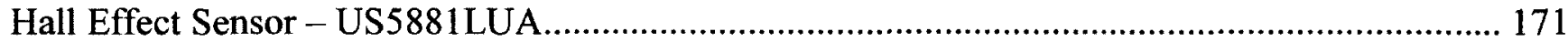

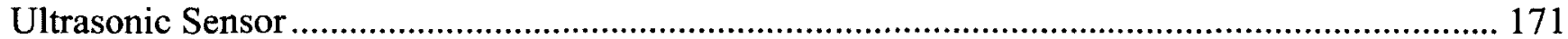




\section{LIST OF TABLES}

Table 3.1: Comparison of ZigBee with other wireless protocols

Table 3.2: The different frequency bands and corresponding characteristics of ZigBee 30

Table 3.3: Prototype components used for the study and their cost. 46

Table 3.4: Prototype components used for the research

Table 3.5: ZigBee starter Kits 50

Table 3.6: The specification of XBee series 1 and series 2 53

Table 3.7: XBee adapter board 56

Table 3.8: SparkFun XBee Explorer USB board 59

Table 4.1: Components and their voltage ranges. 62

Table 4.2: Structure of ports for microcontroller PIC $16 \mathrm{~F} 876$ 66

Table 4.3: Pin assignment for the XBee and XBee PRO modules [61] 74

Table 4.4: List of software used for the research .78

Table 4.5: UK1300 - USB PIC Programmer. 83

Table 5.1: Comparison of our research values with Google and yahoo websites weather report 95

Table 5.2: Location and sensor used to collect data in the apartment 100

Table 5.3: Transition states of sensors 101

Table 5.4: Sequence of events of Activities of daily living of an occupant

Table 7.1: Comparison of ZigBee with other wireless technologies [67] 157

Table 7.2: Features of XBee 158 


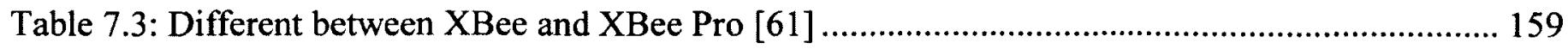

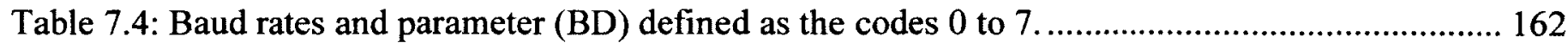

Table 7.5: USB and RS 232 XBee Development Board …............................................................... 163

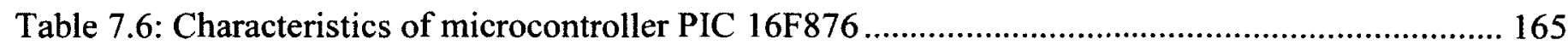

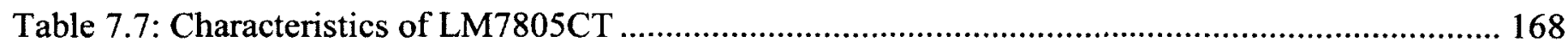

Table 7.8: Characteristics of the piezoelectronic buzzer ................................................................. 169

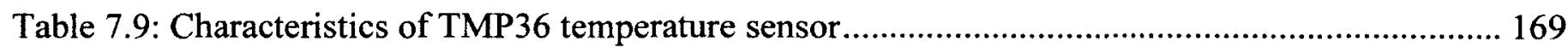

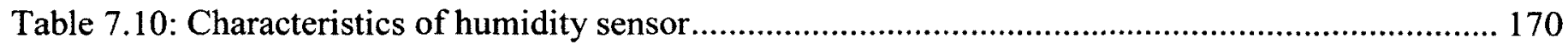

Table 7.11: Characteristics of the photocell sensor ............................................................................ 171

Table 7.12: Characteristics of the Hall Effect sensor ......................................................................... 171

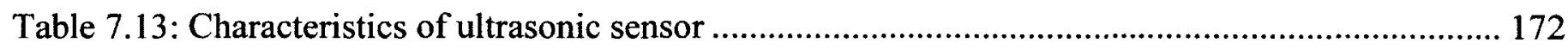




\section{LIST OF FIGURES}

Figure 2.1: Bed-based pressure sensing system architecture of the smart home monitoring system ......... 7

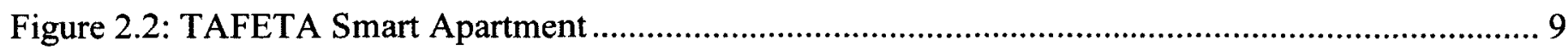

Figure 2.3: Sensor node, including processing board, radio frequency transceiver, and battery ............. 10

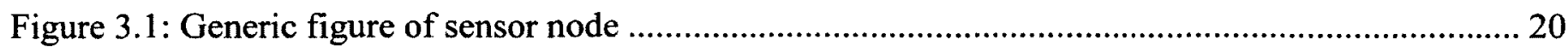

Figure 3.2: Three types of ZigBee nodes: coordinators, routers, and end devices ................................. 31

Figure 3.3: Three common ZigBee topologies: Star, Mesh, and Cluster Tree ....................................... 32

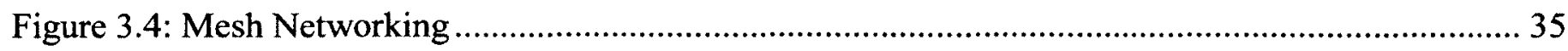

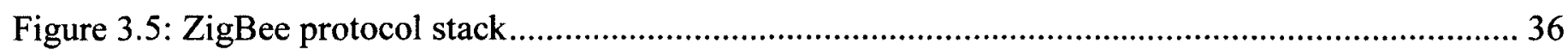

Figure 3.6: Data transfer to a coordinator in IEEE 802.15.4 .......................................................... 40

Figure 3.7: IEEE 802.15.4 functioning mode ………......................................................................... 40

Figure 3.8: Generic figure of base station and remote station of the prototype system ......................... 43

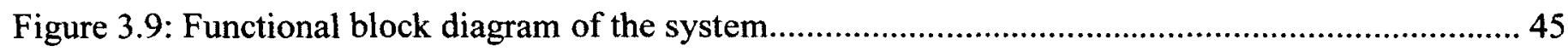

Figure 3.10: XBee Series 1 with RPSMA connector antenna ............................................................ 51

Figure 3.11: XBee Series 2 module with an integrated whip antenna ................................................... 53

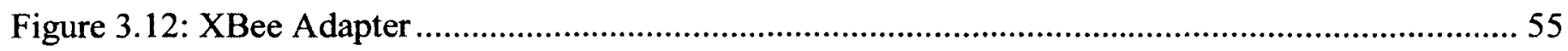

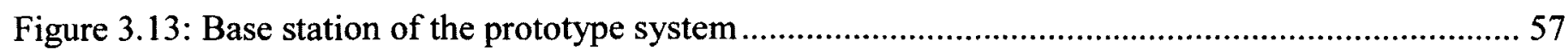

Figure 3.14: SparkFun XBee Explorer USB board ........................................................................... 58

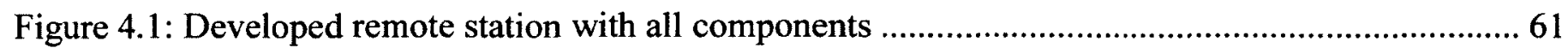

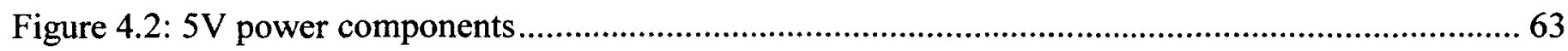


Figure 4.3: $5 \mathrm{~V}$ regulator circuit designed board when the switch is off and on

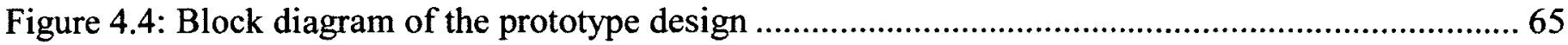

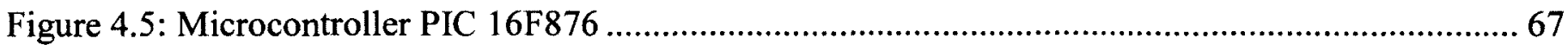

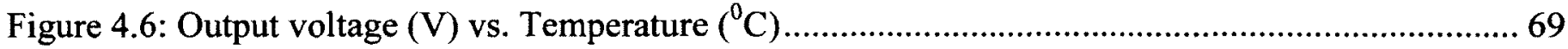

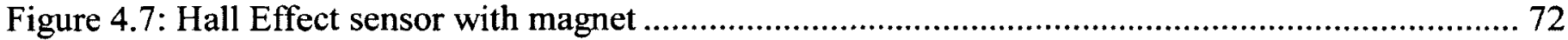

Figure 4.8: System data flow diagram in a UART-interfaced environment ................................. 73

Figure 4.9: Pin details of PIC 16F876 with system components ................................................ 75

Figure 4.10: Remote station including sensors, LCD, microcontroller, buzzer, and XBee module........ 76

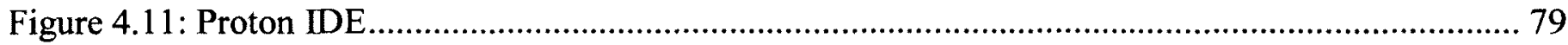

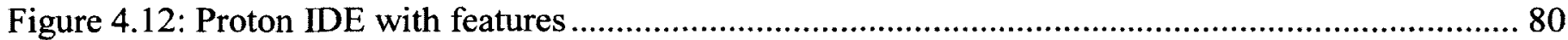

Figure 4.13: HEX file to be loaded into PICKit2 .............................................................. 81

Figure 4.14: PICKit2 is used to load the hex file into the microcontroller via microcontroller programmer hardware

Figure 4.15: PICKit2 is used to load the hex file into the microcontroller via microcontroller programmer hardware 82

Figure 4.16: UK1300 - USB PIC Programmer for programming microcontroller 83

Figure 4.17: USB PIC Programmer connected to computer to program microcontroller 84

Figure 4.18: X-CTU Windows graphical user interface (GUI) for configuration and testing 86

Figure 5.1: Prototype system testing locations at $4^{\text {th }}$ floor of the Minto Building 90

Figure 5.2: Range of XBee Series on 4th floor of the Minto Building 


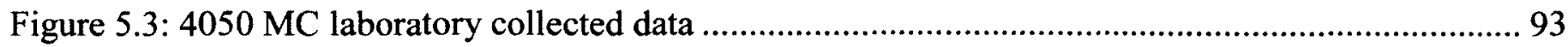

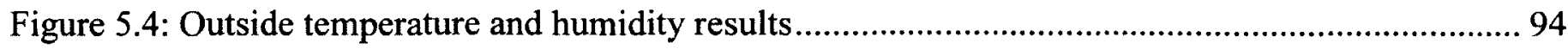

Figure 5.5: Ottawa weather report from Google and Yahoo websites ................................................... 95

Figure 5.6: The system hardware consisted of a remote station and a base station.................................. 97

Figure 5.7: The remote station connected to the electrical outlet ......................................................... 97

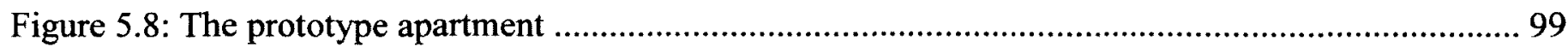

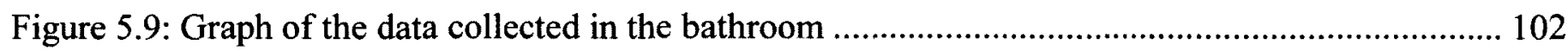

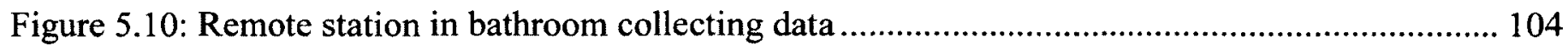

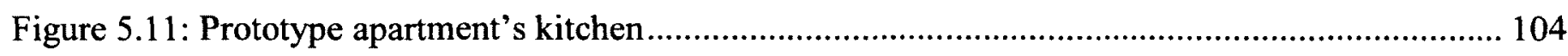

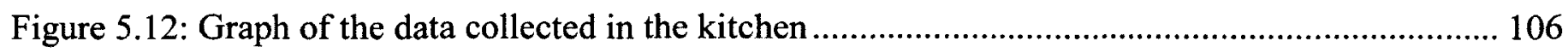

Figure 5.13: Remote station located in the kitchen collecting data ...................................................... 108

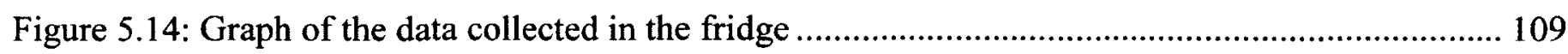

Figure 5.15: Remote station inside fridge collecting data ............................................................... 110

Figure 5.16: Graph of data collected from the living room of the apartment ...................................... 111

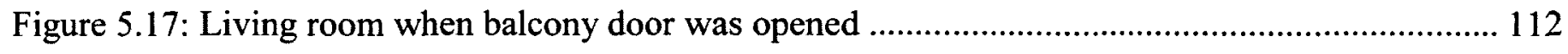

Figure 5.18: Graph of data collected from outside of the apartment .................................................... 114

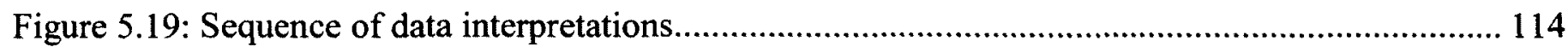

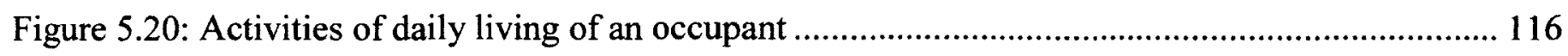

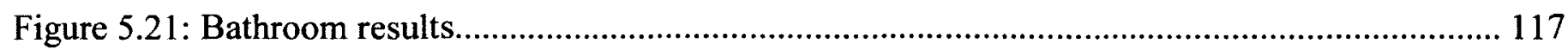

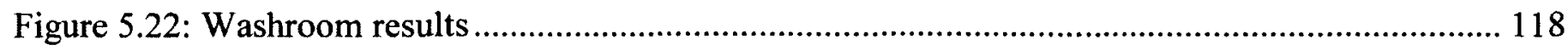


Figure 5.23: Kitchen results

Figure 5.24: Living Room results 120

Figure 5.25: Bed Room results 121

Figure 5.26: Energy consumption due to activities of daily living of an occupant 124

Figure 6.1: Different types of ConnectPort X gateway 131

Figure 7.1: XBee module with type U.FL connector. 161

Figure 7.2: Digi International Development Boards for XBee 162

Figure 7.3: Microcontroller [69]. 166

Figure 7.4: PIC 18F2520, PIC 16F628, PIC 16F876, and PIC 16F877 167

Figure 7.5: Electrolytic capacitors $(10 \mu \mathrm{F} / 50 \mathrm{~V})$ and ceramic capacitor $(100 \mu \mathrm{F} / 6.3 \mathrm{~V})$ 174

Figure 7.6: Battery with $9 \mathrm{~V}$ regulated DC wall-power adapter and $9 \mathrm{~V}$ battery clip 175

Figure 7.7: TMP36 Temperature 176

Figure 7.8: Testing Temperature Sensor using an ice cube 178

Figure 7.9: Detecting light using multi-meter. 179

Figure 7.10: Kitchen Data Figure (a) before cooking and Figure (b) during cooking. 194

Figure 7.11: Living room Data 195

Figure 7.12: Washroom data, Figure (a) shows data before toilet and Figure (b) after toilet 195

Figure 7.13: Bathroom data, Figure (a) data before shower and Figure (b) data during.. 196 


\section{LIST OF APPENDICES}

Appendix A: Wireless Technologies ..................................................................... 152

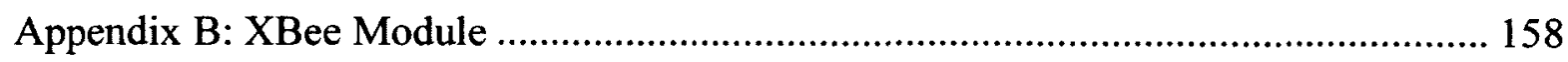

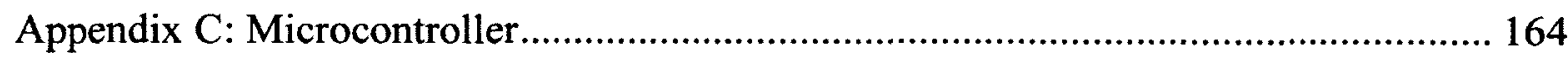

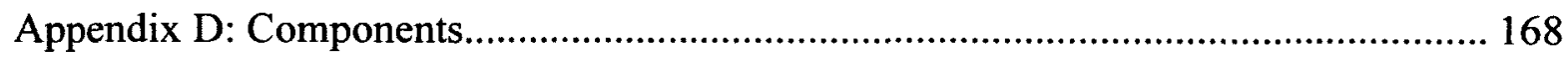

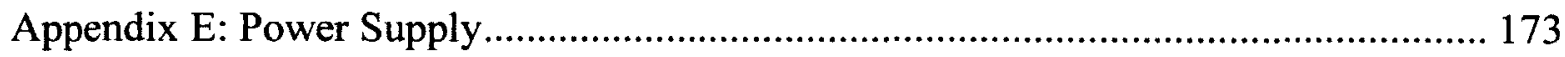

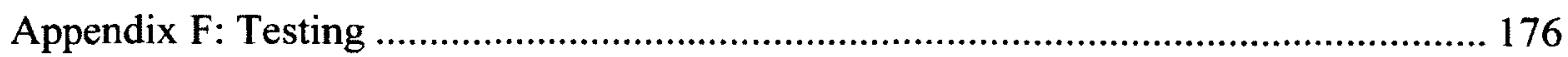

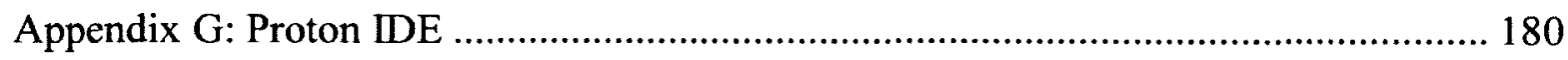

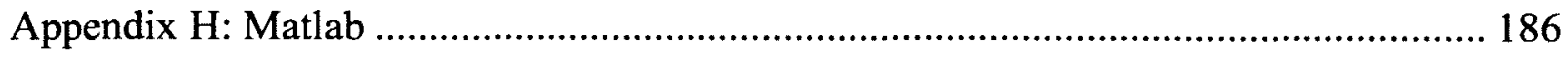

Appendix I: Prototype Apartment Sample Data ........................................................... 194 


\section{CHAPTER 1}

\section{INTRODUCTION}

This chapter describes the objectives, brief overview of the research system technology, contributions, and organization of this thesis.

\subsection{Objectives}

Smart home environments can provide viable alternate living conditions for some aging persons by monitoring the health conditions of the elderly population in real time. Small motors and sensors positioned around the home automatically perform a number of functions, such as opening and closing curtains, turning lights on and off, and raising and lowering sinks and cupboards [1].

The objective of this thesis is to design, develop, and implement a prototype system using smart home technology that utilizes several wireless sensors to remotely detect and monitor an elder's activities of daily living and health condition in their own homes in order to improve the quality of life of the elderly population.

Other purposes of this research are the field testing of the prototype system, utilization of the system to collect

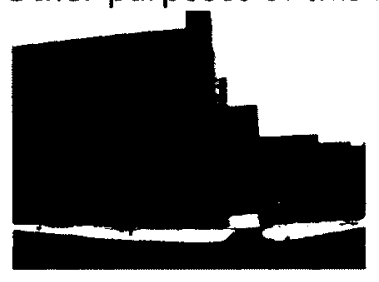
data at different locations and environments, and analysis of the collected data. The field testing of the prototype system was conducted at different locations inside and outside of the Minto Building (the figure to the right) as well as other locations, such as 
the washroom, kitchen, and living room of a prototype apartment. The main goal of this testing was to determine the range of the prototype system and the functionality of each sensor in different environments. After the system was substantiated as operating well in all of the tested environments, data were collected at the different locations and analysed and interpreted in order to identify the activities of daily living of the occupants.

To achieve its objectives, this study sought to:

- Examine current conditions and possible future developments of wireless sensor networks pertaining to the field of home care for the elderly population;

- Investigate the reliability of research to date regarding wireless sensor networks that have been developed in the field of monitoring the health care of occupants in their homes;

- Survey the advantages and disadvantages of the various wireless technologies;

- Design, develop, and implement the prototype system; and

- Collect, analyse, and interpret the data.

\subsection{Brief overview of the research system technology}

In this research, we designed, developed, and implemented a prototype system based on recent advances in low data rate, low-cost, and low-power wireless mesh networking technology. The data rate and capabilities of such technology have been increasing over the past few years, allowing for faster, easier to use, less expensive installation and more reliable wireless connectivity, anytime and anywhere. These advances enable wireless technology to be widely deployed in controlling and monitoring applications. Recent wireless systems consume 
lower power, have a longer lifetime, and use smaller batteries, and mesh networking provides higher reliability and a more extensive range of functions.

\subsection{Thesis Contributions}

This research provides at least four contributions to the field of wireless sensor networks for smart home monitoring environments. The developed prototype system:

(1) Includes distinct features, including: six non-invasive sensors, a microcontroller, a power supply, and XBee modules. Also relies on RF transceivers to establish a wireless communication.

(2) Collects multiple data simultaneously to identify both activities of daily living and usage of appliances at different locations and environments.

(3) Contains a number of safety features to ensure proper operation in the event of emergency. The emergency buzzers alert users of any unacceptable change in behaviour of appliances (such as the fridge) and or status of frames (such as windows or doors).

(4) Enables the analysis and interpretation of the collected data that can potentially identify patterns of behaviour of users and recognize the occurrence of any unforeseen abnormal conditions.

\subsection{Organization of the Thesis}

This thesis is organized into six chapters. Chapter 1 is the introduction. Chapter 2 provides a background about smart home technologies and wireless sensor networking (WSN) technologies and a review of the literature relevant to the thesis, the limitations of the hardware, and past and current smart home research at Carleton University. Chapter 3 concentrates on the design goal, the hardware overview and specifications, and ZigBee 
technology. Chapter 4 illustrates the hardware and software design and the development and implementation of the prototype system. Chapter 5 focuses on data collection, analysis, and interpretations. Finally, Chapter 6 provides the conclusions, limitations, and suggestions for future research. 


\section{CHAPTER 2}

\section{BACKGROUND OVERVIEW}

\subsection{Introduction}

The purpose of this chapter is to provide background information relevant to smart home environments and wireless technologies for the smart home.

The chapter is organized into five main sections. Section 2.2 illustrates a smart home environment. Section 2.3 provides information on past and current smart home research at Carleton University. Section 2.4 explains wireless sensors networks. Section 2.5 reviews the relevant literature and presents the limitations of the techniques usually employed in smart home environments.

\subsection{An Overview of Smart Homes}

A smart home environment is usually equipped with a wireless sensor networks, which is used to relay vital information to a central station. A common definition of a smart home is "a dwelling incorporating a communications network that connects the key electrical appliances and services, and allows them to be remotely controlled, monitored or accessed" [1] [2]; in this context, "remotely" means both within the dwelling and from outside of the dwelling. In other words, a smart home is a living environment that consists of sensors, actuators, networks, and middleware to automatically provide appropriate service to the home's occupants. In other words, a smart home is a house or living environment that contains the technology to allow devices and 
systems to be controlled automatically [3]. It has major usages for environment, security, home entertainment, domestic appliances, information and communication, and health [4], [5]. As its development and applications become increasingly relevant to both the engineering and healthcare communities, the field of smart home technology is growing rapidly [6], [7]. There are at least 20 different home labs set up for researching smart homes by research groups such as MIT, Siemens, Cisco, IBN, Xerox, and Microsoft. More than 30 appliances, at least 5 network protocols, and over 3 artificial intelligence techniques have been used to conduct the research [2]. From a data transmission point of view, smart home technology can be categorized into three areas [2], [8]: Power line, Busline, and Wireless communication.

- Power line: This type of system is made of devices that can be directly connected into the main power supply of the building. The mainstream protocols in Power Line Communication (PLC) technology [2], [8], [9] are X-10, INSTEON, PLC-BUS, LonWorks, and Home Plug.

- Busline: This system uses a separate 12-volt cable (twisted pair cabling) to transmit and receive data from the devices. The cable is similar to that used for phone and network service. The mainstream protocols in Busline communication (BLC) technology are EIB, Cebus, Lonwork, and Batibus [2], [8].

- Wireless communication (Radio frequency (RF) and Infrared (IR) systems): These systems are the networking alternatives that wirelessly exchange data between sender and receiver, but at a much lower data rate than LAN/WLAN. The representative protocols in this case are Bluetooth, ZigBee/IEEE 802.15.4, or Z-wave [2], [8]-[10].

\subsubsection{Radio Frequency (RF)}

Radio frequency (RF) transmission is more suitable for our application, due to the following reasons: 
- Mobility: There is no need for a cable to the location where the display is placed.

- Installation speed and simplicity: Installation of additional sensors to various locations can easily be achieved.

- Cost effectiveness: New installation is inexpensive.

- Topology: Wireless transmission provides extra flexibility in regards to the connection method.

\subsection{Smart Home Research at Carleton University}

Several smart home projects are currently in operation at Carleton University - Canada's Capital University ${ }^{1}$.

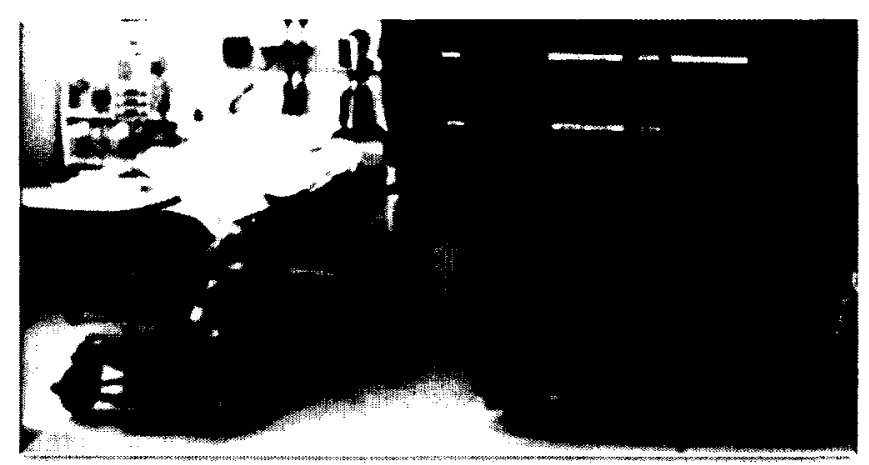

Figure 2.1: Bed-based pressure sensing system architecture of the smart home monitoring system ${ }^{2}$

One of the major concentrations of these projects is on developing sensor networks based on solutions for health care and patient monitoring. These projects incorporate various kinds of sensors technologies, such as

\footnotetext{
${ }^{1}$ Source: http://www.sce.carleton.ca/dept/index.shtml

${ }^{2}$ Figure 2.1 is adapted (in December 2012) from the Department of Systems and Computer Engineering at Carleton University, website: http://www.sce.carleton.ca/dept/index.shtml
} 
wearable sensors, ambient monitoring, or embedded sensors. Moreover, within the home, "smart" or "intelligent" devices include bed-based optical pressure sense system (shown in Figure 2.1) that track sleeping patterns to detect central Apnoea3 [11]-[15], a system to monitor physiological signals caused by chest movement [16], [17], and a device to measure bed-departure time caused by sit-to-stand pressure [18]-[20]. The smart home includes chest belt devices to measure breathing rate, heart rate, and skin conductance during exercise [21]-[23] systems to track food spoilage in fridges [24], [25]. Carleton University is also currently involved in joint projects with other universities, healthcare institutions, and industries; an example of such a project is the TETAFE research, discussed in section 2.3.1.

\subsubsection{Technology Assisted Friendly Environment for the Third Age (TAFETA)}

References [26]-[33] present current research involving Carleton University in a joint Ontario-based project entitled Technology Assisted Friendly Environment for the Third Age (TAFETA). The co-lead in this project is SCO Health Service, one of Canada's largest providers of continuing care, rehabilitation, palliative care, and primary care for elderly persons. The project's other members include other healthcare institutions, schools, and industry partners in Ontario, Canada. Its primary mission is to identify and develop technology that will provide a safe living environment that is responsive to the health needs of elderly persons. The objective of this research is to introduce TAFETA's current smart home research. It describes the various sensing technologies currently under investigation in the groups "Independent Living Apartment" laboratory, called the TAFETA Smart Apartment at the Elisabeth Bruyère Hospital (shown in Figure 2.2). At least 50 patients have used the apartment facilities to date.

3 Apnoea is the most prevalent type of breathing disorder (SDB). 


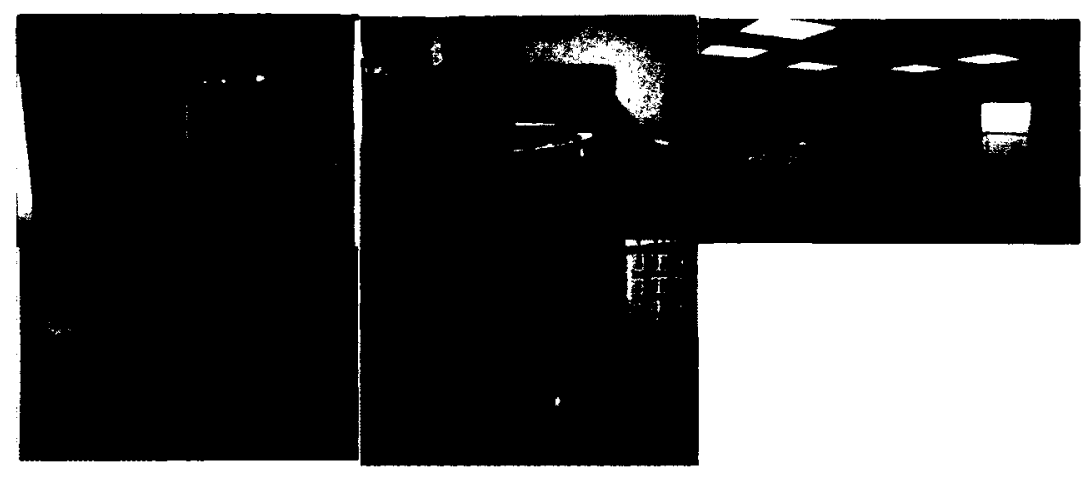

Figure 2.2: TAFETA Smart Apartment ${ }^{4}$

The TAFETA prototype apartment is equipped with several sensors, including magnetic switches to monitor entry into, and exit out of, rooms, thermistors to monitor proper temperature range, accelerometers to detect high impact, radio frequency identifications (RFID) to retrieve misplaced objects, infrared motion sensors to track the presence of motion by the occupant, microphone arrays to detect abnormal sound or calls for help, smart grab bars to aid occupant in the shower and washroom, pressure sensitive mats to monitor occupant's condition at rest, and electronic noses to track food spoilage in the fridge [24], [25].

\subsection{Wireless Sensors Networks}

The wireless sensor networks (WSNs) is one of the key technologies in the smart home design life cycle. The WSNs in the smart home environment can be thought of as a group of spatially dispersed and dedicated sensors for monitoring and recording the physical conditions of the environment and organizing the collected data at a central location [34]. The WSNs measures environmental conditions such as temperature, sound, vibration,

\footnotetext{
t The figure is adapted (in December 2012) from the TAFETA website: www.tafeta.ca
} 
pressure, humidity, wind speed and direction, pollution levels, motion, light intensity, and proximity to objects. The WSNs is made up of a set of independent sensor nodes, gateways, and software.

\subsubsection{Nodes, Gateways, and Software}

The sensor nodes are "self-contained" units, each consisting of a radio frequency (RF) transceiver usually with a single omni-directional antenna, one or many sensors, a low-speed on-board processor (microcontroller, CPUS, or DSP chip), and an energy source (usually a battery or solar cells). Figure 2.3 shows a sensor node. Sensor nodes communicate wirelessly and often self-organize after being deployed in an ad-hoc fashion [35].

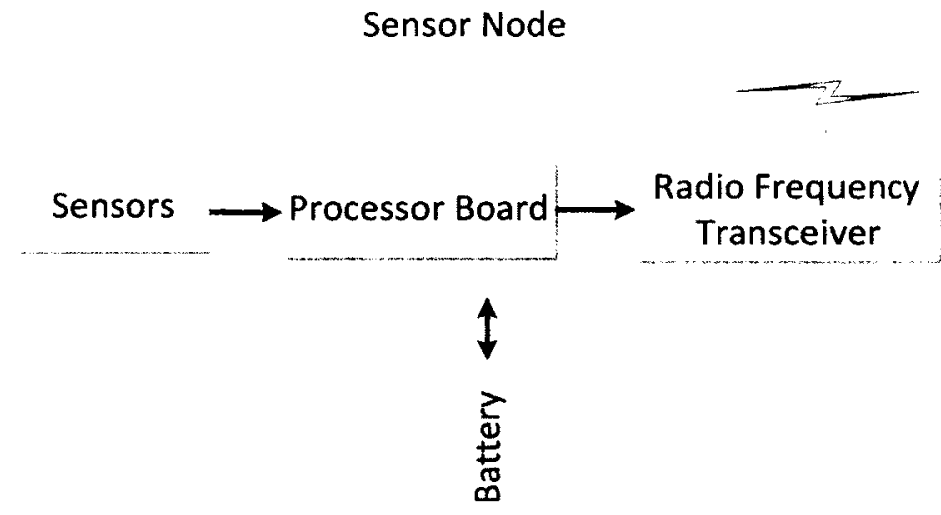

Figure 2.3: Sensor node, including processing board, radio frequency transceiver, and battery

Each node can perform self-diagnosis, which means it can relay data off other nodes within its range until the information reaches its final destination [36]. The range is extended by allowing data to hop from node to node, and reliability is increased by "self-healing," which is the ability to create alternate paths when one node fails or a connection is lost [37]. In addition, when data is transmitted from one node to another, a network-level 
acknowledgement is transmitted back across the established route to the source node. This acknowledgement packet indicates to the source node whether or not the data packet was received by the destination node. If a network acknowledgement is not received, the source node will re-transmit the data [38].

\subsubsection{Wireless Sensors Networks in Healthcare}

Wireless sensor networks are an emerging technology [39] consisting of small, low-power, and low-cost devices that integrate limited computation, sensing, and radio communication capabilities. The technology has the potential to have an enormous impact on many aspects of emergency medical care [40]. The typical applications of the wireless sensor biomedical networks (WSBNs) are numerous, including smart home healthcare monitoring, medical device networking for emergency call systems, and data collection for patient monitoring, as detailed below.

\subsubsection{Smart Home Healthcare}

WSN technology allows home healthcare patients to utilize a variety of monitoring devices, such as stethoscopes, glucose meters, and sphygmomanometers (blood pressure), in order to collect data. The data collected from these monitoring devices are securely sent to a medical lab over a cellular network to be analyzed. For example, reference [41] proposed wireless sensor biomedical networking to help older people or patients with chronic disorders by increasing their chances of survival ability; at the same time reduce the time of routine check-up and it's real-time monitoring also allows emergency situation to be handled immediately in the hospital. 


\subsubsection{Emergency Call Systems}

Emergency call systems are equipped with WSNs, which allows the residents to call for help if they seek any kind of emergency assistance.

\subsubsection{Patient Data}

In the medical field, WSN technology is used to collect patient's data for medical reasons or to gather additional information for research purposes. WSN technology is embedded in the patient's bedside to facilitate making patient's data widely available in real time for proprietary clinical applications and databases and to eliminate manual data entry. An example of the WSNs in the medical field can be found in a study of sleep patterns to detect apnoea in older adults [11]-[15], which measured chest movement to monitor physiological signals [16] [17] and sit-to-stand timing and symmetry to measure bed-departure timing [18]-[20]. 


\section{CHAPTER 3}

\section{HARDWARE OVERVIEW AND SPECIFICATION}

\subsection{Introduction}

This chapter is organized into thirteen sections: Section 3.2 to 3.4 discusses a literature review and describes hardware limitations as outlined in the literature; the design goal; and an explanation of the wireless technologies. Section 3.5 to 3.8 describes the ZigBee protocol, the topology network; ZigBee outlines the protocol architecture; and a system overview. Section 3.9 to 3.12 provides prototype system overviews, system components, remote station hardware and specifications, and hardware specifications. Lastly section 3.13 to 3.15 explains ZigBee starter development kits, XBee modules, and the base station of the design system.

\subsection{Literature Review}

This section is dedicated to providing an overview of the relevant literature. The relevant literature is divided into seven streams: wireless sensor networks for home healthcare, XBee wireless sensor networks for temperature monitoring, the design of wireless sensors networking node on ZigBee, the development of a PICBased wireless sensor, ZigBee-Based wireless personal area networks for health monitoring, and hardware limitations. 


\subsubsection{Wireless Sensor Networks for Home Health Care}

In reference [42], C.R. Baker et al. present the results of research project where several prototypes for homehealth care monitoring were designed. Two of which were dedicated to the design of a wireless sensor system to monitor sleeping position and general health of an infant. The prototypes detected the sleeping position of the infant in order to protect them from Sudden Infant Death Syndrome (SIDS). Due to their tiny nature, premature infants are susceptible to a variety of health problems. An integrated health monitoring device was also developed, and contained within a swaddling baby wrap with strategically placed sensors, to monitor three main factors: temperature, hydration, and pulse rate [42].

\subsubsection{XBee Wireless Sensor Network for Temperature Monitoring}

In reference [43], Boonsawat et al. explain an embedded wireless sensor network (WSN) prototype system for temperature monitoring in the management of air-conditioning systems at the Sirindhorn International Institute of Technology (SIIT) in Thammasat University, Pathum-Thani, Thailand. The ultimate goal of the project was to help save energy costs and reduce energy consumption in the building. The system was designed to provide a web user-interface to access the current and past temperature readings in different rooms of the building. The designed WSN system is comprised of a data gateway or a coordinator that wirelessly joins each WSN temperature-monitoring node located in each room. Each WSN node consists of a microcontroller on an Arduino ${ }^{5}$ board and an XBee wireless communication module based on the IEEE 802.15.4/ZigBee standards. The

\footnotetext{
${ }^{5}$ Arduino is an open-source electronics prototyping platform based on flexible, easy-to-use hardware and software, source: http://www.arduino.cc/
} 
coordinator also has an Ethernet interface and runs a simple data web server. Hence, the coordinator allows data collection over XBee and data access from web browsers.

\subsubsection{Design of Wireless Sensors Networking Node on ZigBee}

In reference [44], S. Veerasingam et al. propose a portable wireless data-acquisition system for temperature in real time process dynamics. Process variables such as temperature, pressure, flow, or level vary with time in certain applications and such variation is recorded so that a control action can take place at a defined set point. The study proposed an 8 bit embedded platform for a sensor with a network interface using the 802.15.4 ZigBee protocol that is specially designed for the sensor networks. This wireless data logger senses and monitors variations in local temperature and transmits the data within the range to an assigned embedded processorbased server. Received temperature is displayed on a local Liquid Crystal Display (LCD) on the assigned server and simultaneously on a central computing unit placed within range. The central base station receives the data, stores it in the file, and plots the variations.

\subsubsection{Development of a PIC-Based Wireless Sensor}

In reference [45],Yussoff Y. et al. demonstrate the performance of WSN models that were developed using temperature sensors, a microcontroller, and XBee technology. The project aimed to use the wireless sensor network to compare the actual temperature in different environments and collect data transferred by the sensor. The collected information from the temperature sensor was processed by the microcontroller before it was transmitted to the base station. The microcontroller was programmed to send the data periodically to the base station through the XBee module. Temperature results were displayed at the base station. 


\subsubsection{Performance Study on ZigBee-Based Wireless Personal Area Networks for Health Monitoring}

In reference [46], B. K-P. Koh and P-Y. Kong conducted research on the use of ZigBee wireless personal area networks (WPANs) for real-time health (heartbeat) monitoring to determine an increase in packet delays due to contentions and collisions in transmissions when multiple ZigBee WPANs are in close proximity to each other. They derived mobility patterns from the analysis of a real-time video trace and subsequently estimated the delay performance from the video trace by combining data collected from the ZigBee experiments. As such, for real-time heartbeat monitoring, a WPAN needs to ensure a 300 milliseconds packet delay so that a systole reading can be captured before the next one is generated. The results of the study showed that the requirement of a 300 millisecond packet delay is not met only $11 \%$ of the time. Also, when failure occurs, it will last for an average duration of 1.4 seconds.

\subsubsection{Temperature Data Logger Using IEEE 802.15.4/ZigBee Protocol}

In reference [47], Sehgal el al. propose an 8 bit embedded platform for a sensor with a network interface using the 802.15.4, ZigBee protocol to monitor the variations in local temperature and transmit the data within the range to an assigned embedded processor based server. Received temperature is simultaneously displayed on a local liquid crystal display (LCD), an assigned server, and a computer.

\subsubsection{Limitations of Hardware}

From the literature review, it can be noted that most of the techniques that have been implanted for the smart home environment have limitations. As a result of these limitations, it would be difficult to gather information 
needed for our research using the existing techniques. These limitations can be grouped into four categories: number of sensors, power usage, price, and performance.

- Number of sensors: Most of the existing techniques are limited to one or two sensors that are focused on one specific objective (e.g., monitoring either temperature, humidity, or motion).Sometimes, it is difficult to obtain reliable information with just one or two sensors. For example, temperature sensors can be suitable for providing information on appliances, but not on other areas, such as monitoring entry into, or exit out of, rooms or buildings.

- Power usage: Most of these techniques are off-the-shelf. They are equipped with 2 AA batteries. Depending on the application, for some activities the battery lifetime could last a few days. This can be problematic for us because our applications require long battery lifetimes to enable the continuous monitoring of Activities of daily living of occupants.

- Price: As indicated above, most of these devices are off-the-shelf and are too expensive for the context of our research.

- Performance: Most of these systems were developed to accommodate the customer. The devices are not flexible; making them "intelligent" in the context of a precise application, such as the monitoring of the elderly population, is a very difficult task. In addition, some sensors do not perform well in some situations. For example, motion sensors can be deceived by warm objects and motionless persons. Ultrasound can be misled by any object. Also, as mentioned above, at times, it is difficult to obtain reliable data with just one sensor.

- Need for "intelligent" approaches for elderly monitoring: Aging populations living alone in their home present at least two major associated risks: short-term risks, such as collapse, falling, or stroke, and 
long-term risks, such as loss of independence, malnutrition, or insufficient hygiene. Moreover, any abnormal events that happen to an aging person in a home can lead to more serious illnesses or even death. There is a need for technique that can monitor any change in behaviour of the Activities of daily living of these aging populations in their homes to prompt early and appropriate medical or welfare intervention. Furthermore, according to gerontologists, identifying changes in everyday behaviour, such as sleeping, food preparation, housekeeping, and entertainment, is often more valuable than biometric information for the early detection of emerging physical and mental health problems, particularly for the elderly [48], [49].

In reviewing the existing techniques, we have concluded that there is a need to design and develop techniques that meet the needs of the elderly population and achieve high levels of accuracy, while also being portable, easy to use, and cost-effective. The necessary system would be able to continuously monitor relatively simple parameters to measure the interaction between participants and their environment.

\subsection{Design Goals}

Unlike old-style networks, sensor design networks are influenced by many factors. The core factors that need to be understood as a first step to making an informed choice are: environment, fault tolerance, transmission media, hardware constraints, sensor node size, power consumption, expandability, network topology, product cost, and security and different methods and standards of wireless communication. It is important to examine these factors when designing sensors because they serve as guidelines for the design of a protocol or an algorithm for wireless sensor networks. In addition, these influencing factors enable the comparison of different 
schemes [50], [51]. To deal with the technical challenges of designing an efficient wireless network based on multi-sensors for the smart home, the following design goals need to be satisfied.

\subsubsection{Environment}

Sensor networks are expected to be able to operate within different environments, from hospitable to extremely hostile. They must be autonomous and operate in high volumetric densities unattended. The system must have fault tolerance to respond gracefully to unexpected hardware or software failure.

\subsubsection{Fault tolerance}

There are several levels of fault tolerance. The level of fault tolerance should meet the environment to which the system will be exposed. For example, remote stations deployed in a hospitable environment, such as in a house, monitoring temperature, humidity, or light may require low tolerance levels to continue to operate compared to those deployed in an extremely hostile environment, such as around machinery or in a battlefield, ocean bed, factory, or disaster or toxic area.

\subsubsection{Transmission Media}

Remote stations in the wireless sensor network communicate with each other through transmission media. These communication media can be established through infrared, radio, or optical radios. There are advantages and disadvantages to each of these media. The optical communication medium consumes low amounts of energy and provides high levels of security; however, it requires line-of-sight and is sensitive to atmospheric conditions. The infrared communication medium does not require an antenna to operate, but it has limited 
broadcasting capacity. The radio communication medium is the best choice of the three communication media even though it requires an antenna. One option for the radio communication medium is through industrial, scientific, and medical (ISM) bands, which are unlicensed bands and bands allocated for low power devices. To guarantee that the spectrum is used fairly, the unlicensed bands need to follow national usage requirements.

\subsubsection{Hardware Constraints}

The main functions of the sensor node network (remote station) in the field are to detect events, process data, and then transmit the data to the base station. The remote station is made up of the following four basic components (as shown in Figure 3.1): sensing unit, processing unit, transceiver, and power unit.

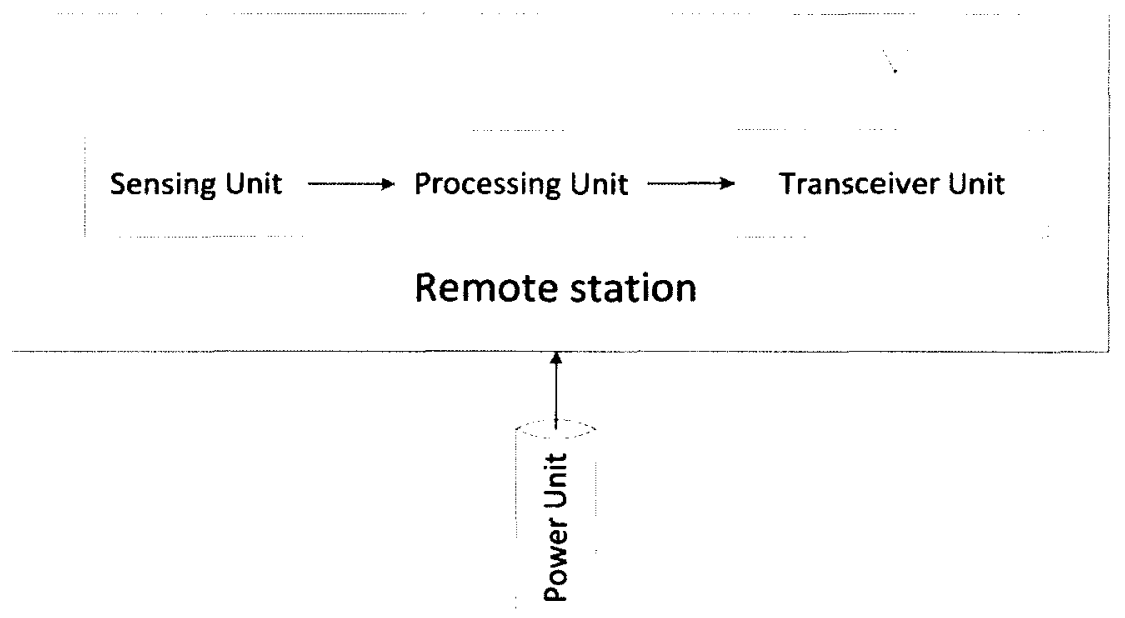

Figure 3.1: Generic figure of sensor node

- Sensing unit: The sensing unit is composed of sensors. Sensors are devices that detect or measure a physical condition (such as heat, light, sound, pressure, magnetism, or motion). The analog voltage or signals generated by corresponding sensors based on the observed phenomenon are converted to 
digital form by analog-to-digital conversion ( $A D C)$, processed, and then transmitted to the processing unit for further processing analysis. These tasks are the major sources of power consumption in the sensing unit.

- Processing unit: The processing unit can be a microcontroller or microprocessor. Its main responsibilities include responding to the sensing unit, processing data, and controlling the functionality of other components in the remote station.

- Transceiver: The transceiver unit is used to connect the remote station to the network. Within the transceiver, a source of power consumption for radio usage in the remote station evolves in five modes: transmit, receive, idle, command and sleep.

- Idle mode: The RF module (node) is in Idle mode when it is not receiving or transmitting data.

- Transmit mode: The RF module (node) is in Transmit mode when it is transmitting data.

- Receive mode: The RF module (node) is in Receive mode when it is receiving data.

- Sleep mode: The RF module (node) is in Sleep mode when it is not being used and enters lowpower consumption.

- Command mode: The RF module (node) is in Command mode when it is modifying or reading the RF module parameters.

Possible wasteful energy consumption in sensor node could be due to the following reasons:

- Idle: Idle attending to the channel waiting for possible traffic.

- Retransmitting of data: Due to the occurrence of a collision

- Overhearing: When a sensor sends a packet to the wrong recipient. 
- Over-emitting: When sensors receive data when they are not ready for them.

It is recommended to avoid using the idle mode when it is not transmitting or receiving data to reduce power usage in the unit.

- Power unit: Power consumptions can be divided into three categories: sensing, communicating, and data processing. A more detailed discussion of this unit can be found in the power consumption section.

\subsubsection{Sensor Node Size}

Since the sensor nodes are deployed in the hundreds or thousands, they are designed to be small with limited resources such as energy, computational power, and available storage. All of the components in the remote station may need to fit into a matchbox-sized module [52]. The required size may be even smaller than a cubic centimeter [53].

\subsubsection{Power Consumption}

Advances in microelectronic technology have made it possible to design sensor nodes of small physical size, on the order of one cubic centimeter [53]. Although the size has made the wireless sensor network usable in many different applications, at the same time the size affects certain resources such as energy, computational power, and available storage. Sensor nodes are equipped with a limited energy source: batteries. Their lifetime, therefore, depends on the lifetime of the batteries. Depending on the application, the lifetime of the sensor node can be only few days, which is unacceptable in areas of application that require constant monitoring. In addition, hundreds or thousands of wireless sensor networks are often deployed in harsh and hostile 
environments. In these contexts, after the deployment phase occurs, it is difficult to have access to the nodes to recharge or replace batteries.

In some applications (such as home automation or Smart energy) where line power is readily available in places where the sensors nodes are placed, the line power can be used so that sensor nodes can have an unlimited supply of power resources for the applications. In this respect, the nodes are partially a wireless system. Although the nodes are not portable, the data transmission occurs in a wireless manner.

\subsubsection{Expandability}

Depending on the application, the number of sensor nodes deployed in the field may be in the order of hundreds or thousands. New scheme systems must be able work with this numbers of stations. The design goal is to develop flexible and scalable architecture that can accommodate the requirements of new sensor nodes in the same infrastructure.

\subsubsection{Network Topology}

The topology network is important; it is the structure that allows standard wireless devices to have multiple simultaneous connections. It is also one of the ways that the different wireless standards are distinguished. In theory, a wirelessly enabled device can connect to any compatible wireless devices within transmission ranges. It is important to understand what the new device will be connecting to. Network topology is an important feature of device connectivity. Not only does it allow devices to have multiple connections, but it also allows devices to join and leave the network at any time. Network topology is one of the important methods to 
distinguish the diverse standards of wireless connections. In short, topology is a method of making and securing connections, managing power consumption, transmitting different types of data, and coexisting with other radios. Remote stations are deployed unattended in high volumetric densities and harsh environments. The stations are prone to frequent failure and make topology network maintenance a significantly challenging issue. Topology maintenance and changes can be viewed in three phases [50], [51]: pre-deployment and deployment, post-deployment, and re-deployment of additional nodes.

\subsubsection{Pre-deployment and Deployment}

In the pre-deployment and deployment phase, the systems for initial deployment must [50], [51]:

- Reduce the installation cost;

- Eliminate the need for any pre-organization and pre-planning;

- Increase the flexibility of the arrangement; and

- Promote self-organization and fault tolerance.

\subsubsection{Post-deployment}

The common issues that affect sensor node topologies after deployment that should be avoided are usually related to improper position, reachability due to jamming, noise, moving obstacles, energy availability, or malfunctioning of the nodes [50]-[52], [54]. These issues should be taken into account. It must be recognized that node failure could be cause by inefficient energy and that the node may be a target for deliberate jamming. In this way, sensor network topologies are prone to frequent changes after deployment [50], [51].

\subsubsection{Re-deployment of Additional Nodes}


Re-deployment of new nodes to replace malfunctioning nodes should be able to be easily achieved and these new nodes should also able to join the network or organize the network without any problem.

\subsubsection{Product Cost}

As we have indicated, the sensor nodes are deployed in the thousands. The price of the sensor nodes is determined by a single sensor node. The price of a single node should as cheap as possible; otherwise it is wise to use a commercial product.

\subsubsection{Standard}

Devices from a diverse range of suppliers need to interact with each other. It is, therefore, important to determine a standard to establish communication to each other's devices. This allows all of the devices that adopt the standard to work together or share elements of their design. Choosing standards is an important task because it has a reference a protocol stack that extends up through enough layers to provide the ability to design interoperable application.

\subsubsection{Security}

Sensor networks can be deployed into any environment, from harsh to hostile. They are not protected from physical attack; anyone can have access to the location where they are deployed. This is major security concern related to the wireless networks because anyone can gain access to sensitive information by listening to transmission messages and can even modify the message and retransmit it to its intended destination.

The following elements of network security are necessary to ensure a secure network [55]: 
- Secrecy or confidentiality: Secrecy provides privacy and protects information from being intercepted. Even if someone intercepts the information, the information would have no meaning.

- Authentication: Authentication methods verify the identity of a person or computer accessing the network.

- Integrity: Integrity maintains data consistency and prevents tampering with information.

- Nonrepudiation: Nonrepudiation provides proof of origin to the recipient.

\subsection{Wireless Technologies}

Besides ZigBee, several other technologies were investigated in terms of their features such as power consumption, distance, data rate, data delivery, cost, learning curve, size, installation, usability, portability, and reliability. The potential wireless technologies that were investigated included Wi-Fi, Bluetooth, Ultra wideband (UWB), Wireless Universal Serial Bus (USB), Infrared (IR) wireless, and XBee. These technologies are discussed very briefly below and their advantages and disadvantages are outlined in the Appendix $A$.

\subsubsection{Wi-Fi}

Wi-Fi is a wireless local area network (WLAN) that uses high frequency radio waves to transmit and receive data over distances of a few hundred feet. The technology is based on the Institute of Electrical and Electronics Engineers (IEEE) 802.11 standards. The main application for Wi-Fi is to provide wireless high-speed Internet and network connectivity. Many computer-associated devices, such as video game consoles, home computer networks, and mobile phones, are supported by Wi-Fi. 


\subsubsection{Bluetooth}

Bluetooth is one of a standard of wireless radio technologies that utilizes short distance radio waves to transmit signals over short distances. Bluetooth technology was originally regarded as a wireless alternative to RS-232 data cables. The technology is suitable for applications that are between different electronic devices, such as cars phones, mobiles, laptops, headsets, and various other devices.

\subsubsection{Ultra wideband (UWB)}

Ultra wideband (UWB) technology operates on the $3-10 \mathrm{GHz}$ frequency band on an unlicensed basis. With regulatory approval in major markets worldwide, this technology has gained broad industry use as evidenced by its selection for the WiMedia standard in addition to Wireless USB and high-speed Bluetooth. UWB is used in wireless networking to achieve high bandwidth connections with low power utilization by using a large portion of the radio spectrum. UWB technology has potential applications in consumer electronics and wireless personal area networks (PAN) ranging from digital camcorders and DVD players to a mobile PCs and a high-definition TVs (HDTV). However, the most recent applications target sensor data collection, precision locating, and tracking applications.

\subsubsection{Wireless Universal Serial Bus (USB)}

Wireless Universal Serial Bus (USB) is an industry standard for wireless networking based on Ultra wideband (UWB) signalling technology. Wireless (USB) is a short-range, high-bandwidth, low-power wireless radio communication protocol that employs a computer's USB ports for wireless local networking for applications such as USB products. 


\subsubsection{Infrared (IR) wireless}

Infrared (IR) is a wireless technology that is used for short-medium-range communications and control devices or systems that convey data through IR radiation. IR wireless technology is utilized for applications such as intrusion detectors; home-entertainment control units; robot control systems; medium-range, line-of-sight laser communications; and cordless microphones, headsets, modems, printers, and other peripherals. Although some systems operate in line-of-sight mode with a visually unobstructed straight line through space between the transmitter and receiver, other systems operate in diffuse mode, also known as scatter mode. These kinds of systems can function when the transmitter and receiver are not directly visible to each other. An example of such a system is a television remote-control box [56]. The remote-control box does not have to be pointed directly at the set, although the box must be in the same room as the set or just outside the room with the door open.

\subsubsection{Radio Frequency Identification (RFID)}

Radio frequency identification (RFID) is rapidly becoming the technology of choice for automatically identifying people or objects. It is small, inexpensive, and not based on line-of-sight operation. A RFID system typical consists of an RFID reader (or transceiver) and an RFID tag (or transponder). The RFID reader components consist of a RFID transmitter, one or more antennae, and an RFID receiver to allow encoded data to be transmitted and received from a tag or tags to be processed. A typical tag may contain a digital microchip attached to antennae to send, receive, and process decoded data. 


\subsubsection{ZigBee}

Based on our investigation, the most suitable wireless technology for this research is ZigBee. The ZigBee technology is widely used in smart home systems. This technology is designed for a low data rate, low power consumption, low-cost, wide coverage, and wireless mesh networking standard communications protocol for wireless smart home control and monitor system. Table 3.1 illustrates comparison of ZigBee to these potential wireless technologies. The comparison is mostly based on the following technical features: range, frequency band, bandwidth, network topology, power, and security.

\begin{tabular}{|c|c|c|c|c|}
\hline & ZigBee & Wi-Fi & Bluetooth & Z-wave \\
\hline IEEE Spec. & 802.15 .4 & $802.11 x$ & 802.15 .1 & N/A \\
\hline $\begin{array}{c}\text { Operating } \\
\text { Frequency Band }\end{array}$ & $\begin{array}{c}868 \mathrm{MHz}(\mathrm{EU}), \\
900-928 \mathrm{MHz} \text { (NA), } \\
2.4 \mathrm{GHz} \text { (Global) }\end{array}$ & 2.4 and $5 \mathrm{GHz}$ & $2.4 \mathrm{GHz}$ & $900 \mathrm{MHz}$ \\
\hline Bandwidth & 20,40 and $250 \mathrm{Kbits} / \mathrm{s}$ & $\begin{array}{c}11 \& 54 \mathrm{M} \\
\text { bits/sec }\end{array}$ & $1 \mathrm{M}$ bits $/ \mathrm{s}$ & $40 \mathrm{Kbits} / \mathrm{s}$ \\
\hline $\begin{array}{c}\text { Range } \\
\text { (meters) }\end{array}$ & $10-100 \mathrm{~m}$ & $50-100 \mathrm{~m}$ & $10 \mathrm{~m}$ & $30 \mathrm{~m}$ \\
\hline $\begin{array}{l}\text { Networking } \\
\text { Topology }\end{array}$ & $\begin{array}{c}\text { Ad-hoc, peer to peer, } \\
\text { star, or mesh }\end{array}$ & Point to hub & $\begin{array}{c}\text { Ad-hoc, very small } \\
\text { networks }\end{array}$ & Mesh \\
\hline Network size & 65536 & 32 & 7 & 232 \\
\hline $\begin{array}{c}\text { Power } \\
\text { Consumption }\end{array}$ & Very low & High & Medium & Very low \\
\hline Security & $\begin{array}{c}128 \text { AES plus } \\
\text { application layer } \\
\text { security }\end{array}$ & & $\begin{array}{l}64 \text { and } 128 \text { bit } \\
\text { encryption }\end{array}$ & \\
\hline Applications & $\begin{array}{l}\text { Control and } \\
\text { monitoring }\end{array}$ & Wireless LAN & $\begin{array}{c}\text { Device } \\
\text { Connectivity }\end{array}$ & $\begin{array}{l}\text { Control and } \\
\text { monitoring }\end{array}$ \\
\hline
\end{tabular}

Table 3.1: Comparison of ZigBee with other wireless protocols

\subsection{ZigBee Protocol}

ZigBee is a wireless communication standard based on IEEE 802.15.4 low-rate wireless personnel area network

(LR-WPAN). The standard was established to meet the needs of low-cost, low-data rate, low-power, and short- 
range unattended devices that accommodate the global standard. The potential applications of the ZigBee include home automations, smart energy, telecommunication, and healthcare. ZigBee is specified to operate in the industrial, scientific, and medical (ISM) radio bands: $868 \mathrm{MHz}$ with one channel and over-to-air date rate of $20 \mathrm{kbps}$ in Europe, $915 \mathrm{MHz}$ with 10 channels and over-to-air date rate of $40 \mathrm{kbps}$ in North America, and $2.4 \mathrm{GHz}$ with 16 channels and over-to-air date rate of $250 \mathrm{kbps}$ for worldwide coverage. ZigBee allows multi-point networks. The different frequency bands and their characteristics are shown in Table 3.2. ZigBee protocol features include [57]:

- Support for multiple network topologies, such as point-to-point, point-to-multipoint, and mesh networks;

- Low duty cycle - provides long battery life;

- Low latency;

- Direct Sequence Spread Spectrum (DSSS);

- Up to 65,000 nodes per network;

- 128-bit AES encryption for secure data connections; and Collision avoidance, retries, and acknowledgements.

\begin{tabular}{|c|c|c|c|c|c|}
\hline Band & Data rate & Modulator & Channels & Coverage & License required \\
\hline $868 \mathrm{MHz}$ & $20 \mathrm{kbps}$ & BPSK & 0 & Europe & No \\
\hline $915 \mathrm{MHz}$ & $40 \mathrm{kbps}$ & BPSK & $1-10$ & North America & No \\
\hline $2.4 \mathrm{GHz}$ & $250 \mathrm{kbps}$ & O-QPSK & $11-16$ & Global & No \\
\hline
\end{tabular}

Table 3.2: The different frequency bands and corresponding characteristics of ZigBee 


\subsubsection{ZigBee nodes}

There are three types of ZigBee nodes: ZigBee coordinator (ZC), ZigBee router (ZR), and ZigBee end device (ZED).

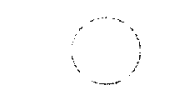

End Device

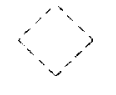

Router

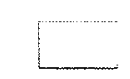

Coordinator

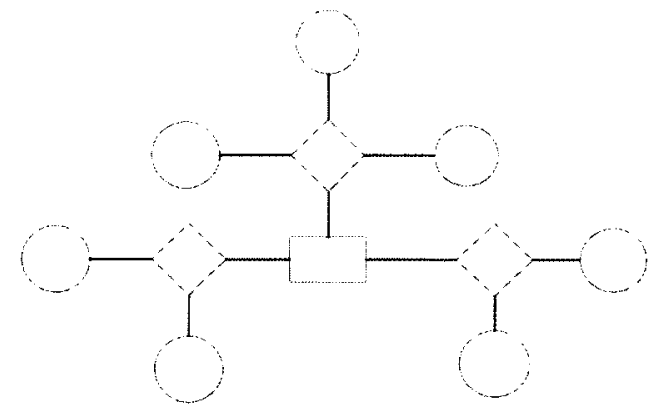

Figure 3.2: Three types of ZigBee nodes: coordinators, routers, and end devices

\subsubsection{ZigBee Coordinator (ZC)}

The ZigBee network consists of one ZigBee coordinator and one or more end device to form the personnel area network (PAN). The end device has a unique PAN identity (known as PAN ID) to avoid miscommunication between PANs. The coordinator PAN ID is established using the ID (PAN ID) and router commands. The end device associates to a coordinator without knowing the address, PAN ID, or channel of the coordinator. It is the responsibility of the ZigBee coordinator to monitor and control the overall network.

\subsubsection{ZigBee Router (ZR)}

The ZigBee router establishes a communication link between the coordinator and the end device. Establishing this communication link is very important in situations that require the relay of vital information from several remote stations (end devices) to central unit (ZigBee coordinator). The ZigBee router not only extends network 
area coverage but also redirects data around obstacles. It also provides up alternative path for data transfer in case of network congestion or device failure.

\subsubsection{End Device (ED)}

The end device must be connected to the coordinator or router in the network. The end device has limited resources to perform its function. The end device can transmit or receive messages, but cannot relay messages from other ZigBee networks. It is simple and consumes less power.

\subsection{ZigBee Topology Network}

The three common network topologies supported by ZigBee are star, peer-to-peer (mesh), and cluster tree. The structures of these topologies are shown in Figure 3.3.

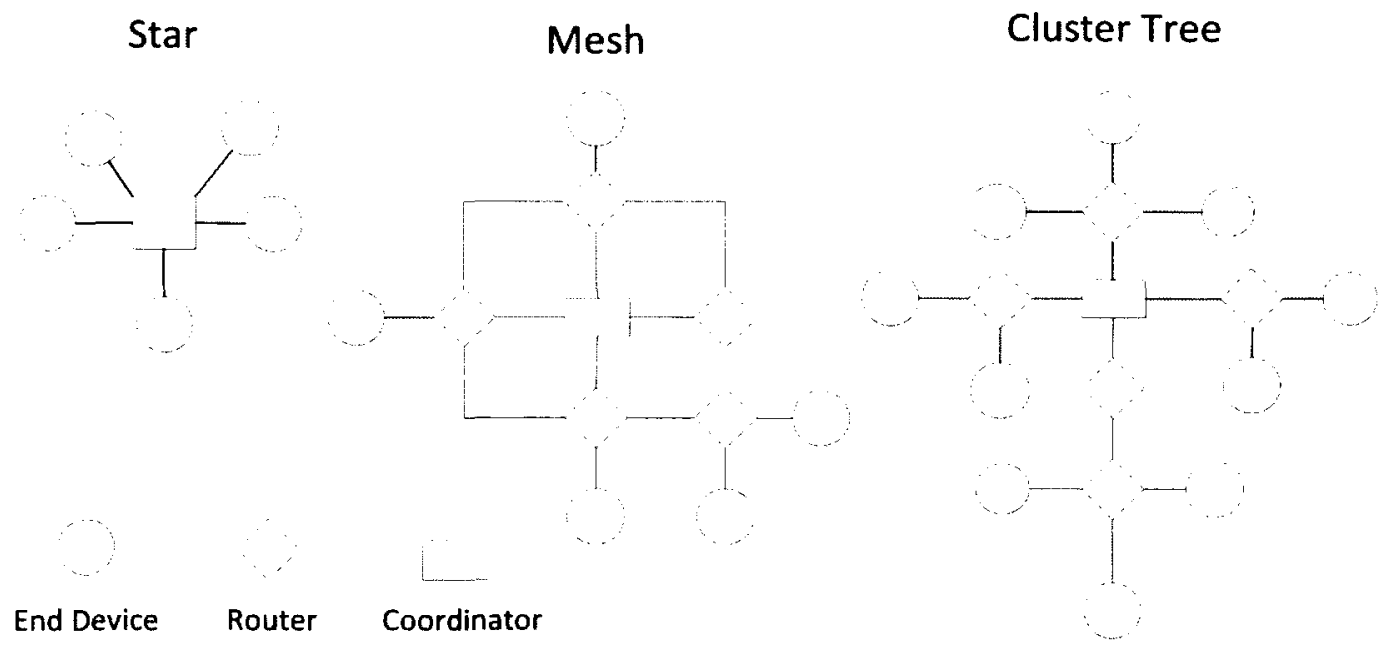

Figure 3.3: Three common ZigBee topologies: Star, Mesh, and Cluster Tree 


\subsubsection{Star Topology Network}

The star topology network consists of one coordinator connected to one or many end devices (ED). The end devices have limited resource to actively transmit data, receive acknowledgement, and return to sleep. They do not relay information from other devices to the coordinator. By spending much time sleeping, the network lowers their duty cycle and extends battery life.

Data in star topology can be transferred in two ways only:

- Data transfer to a coordinator from a device and

- Data transfer from a coordinator to a device.

\subsubsection{Mesh or Peer-to-Peer Topology Network}

Mesh networking is a powerful way to route data. The peer-to-peer topology network consists of all of the routers. In peer-to-peer, each device can communicate directly with any other device if the devices are placed within the range necessary to establish a successful communication link. Range is extended by allowing data to hop node to node and reliability is increased by "self-healing," the ability to create alternate paths when one node fails or a connection is lost [37].

A mesh network has the following characteristics:

- Every node is capable of connecting directly to all of its neighbouring nodes;

- Every node is capable of routing traffic to and from all of its neighbouring nodes;

- The network is self-forming. This means that new nodes are automatically added to the network without the need for manual configuration; and 
- The network is self-healing. This means that the network automatically adjusts the routes to and from nodes.

Data in peer-to peer topology can be transferred in three ways:

- Data transfer to a coordinator from a device;

- Data transfer from a coordinator to a device; and

- Data transfer between two peer devices.

\subsubsection{Mesh Example}

Figure 3.4 (Figure 3.5A) illustrates a typical mesh network, and Figure 3.4 (Figure 3.5B) shows how the mesh network dynamically updates itself when a connection is lost. Node 5 creates an alternative pathway through Node 4 to send collected data to the gateway when the path to the gateway through Node 3 fails for any reason. Node 5 waits for acknowledgement from Node 3. If there is no acknowledgement from Node 3, Node 5 will still attempt to deliver the package to the gateway; however, through the next best path.

In our case, we assumed that the best alternative hop to the gateway is Node 4 . Node 5 will dynamically adapt itself by creating an alternative path through Node 4 to send collected data to the gateway. 


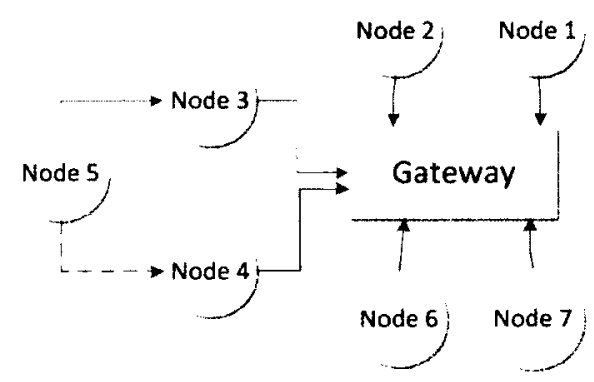

Figure 3.5 A Mesh Network

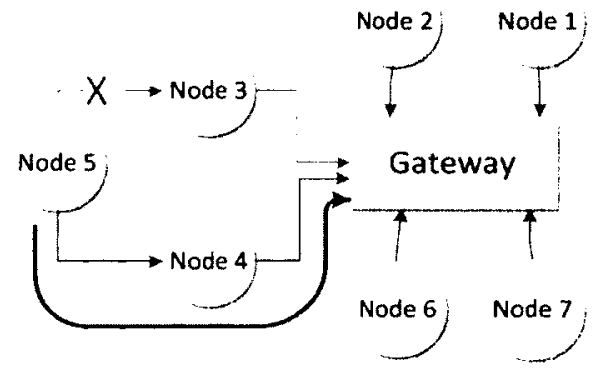

Figure 3.5 B Alternative Route for Node

Figure 3.4: Mesh Networking

The collected data is wirelessly transmitted to the gateway. The gateway collects, processes, analyzes, and presents the measurement data using software. The software running on the gateway performs appropriate computation and displays the information on the user screen.

\subsubsection{Cluster Tree Topology Network}

The cluster tree is a combination of both star and mesh topology. The topology allows for a heterogeneous network in which ZigBee routes and actively and continuously transmits and revives the data that requires a robust power supply, while other nodes respond to stimulus and spend most of their time sleeping. The network is reliable and scalable to extend the range of the network beyond that of a star and mesh. Data in cluster tree topology can be transferred in three ways:

- Data transferred to a coordinator from a device;

- Data transferred from a coordinator to a device; and

- Data transferred between two peer devices. 


\subsection{ZigBee Protocol Architecture}

ZigBee protocol architecture consists of IEEE 802.15.4 and a ZigBee layer, as shown in Figure 3.5.

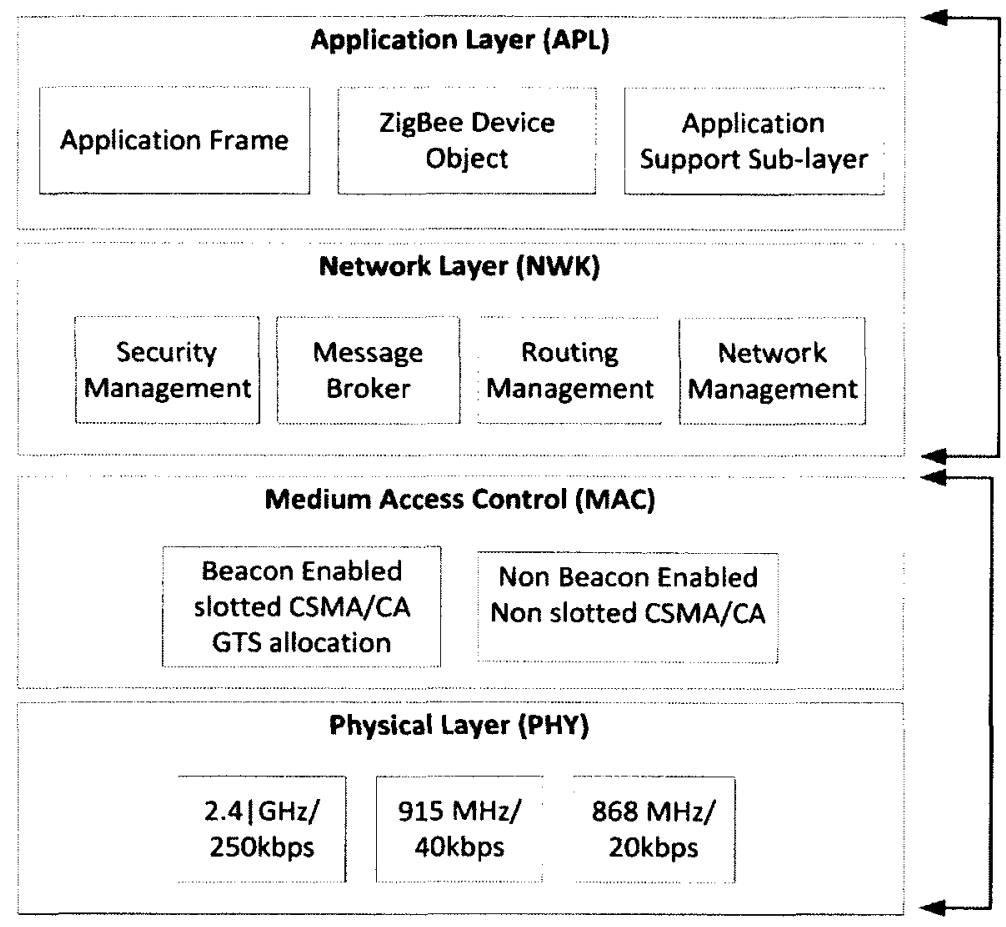

ZigBee Alliance

IEEE 802.15.4

Figure 3.5: ZigBee protocol stack

\subsubsection{IEEE 802.15.4}

IEEE 802.15.4 consists of a physical (PHY) layer and a Medium Access Control (MAC) layer.

\subsubsection{Physical (PHY) layer}

PHY layer is the lowest layer of the IEEE 802.15.4 protocol. It is the closest layer to the hardware. The layer directly controls and communicates with the radio transceiver. The description of the PHY layer outlines a low- 
power spread spectrum radio operating at frequency bands such as $2.4 \mathrm{GHz}, 915 \mathrm{MHz}$, and $868 \mathrm{MHz}$. The layer is responsible for the following tasks:

- Activation and deactivation of the radio transceiver: The radio transceiver can operate in four different modes: transmitting, receiving, sleeping, and idle.

- Energy detection (ED): This layer detects and estimates signal energy (power) level within the bandwidth of an IEEE 812.15.4 channel and determines whether the channel (desired channel) is busy or available (idle) before signal transmission.

- Link quality indication (LQI): This layer assesses the quality of signal on a link.

- Clear channel assessment (CCA): This operation is responsible for reporting medium activity states (busy or idle). It ensures that the channel is not selected by any other device for a signal to be transmitted. The three following tasks are performed by the CCA:

- Energy detection mode: This mode determines whether the received energy is higher than a given threshold (e.g. energy detection threshold). If the received energy is high it is an indication of the channel being busy.

- Carrier sense mode: This mode is an alternative to the busy or idle mode of a frequency channel.

- Carrier sense with energy detection mode: This mode is the combination of the above methods.

- Channel frequency selection: The PHY layer selects the desired channel upon the reception of a request from a higher layer.

\subsubsection{Medium Access Control (MAC) Layer}


The MAC layer provides an interface between the PHY layer and the network layer. The description of the MAC layer explains how multiple 802.15.4 radios operating in the same area can share the airwaves. The MAC layer description also explains different network topologies (e.g. star, peer-to-peer, and Tree cluster). Its responsibilities include the following tasks: generating beacons to enable the network and synchronizing the device in a beacon-enabled network. It is also offers the option for a device to join or leave a network, this option is known as association and disassociation.

\subsubsection{ZigBee Layer}

The ZigBee description explains application profiles that allow devices from different manufacturers to communicate with each other. ZigBee consists of the network layer and the application layer.

\subsubsection{Network (NWK) Layer}

The NWK layer is the interface between the MAC layer and the application frame. It is responsible for managing the network formation and routing. Routing is the process of selecting paths through which the data will be relayed to its destination.

\subsubsection{Application (APP) Layer}

The application layer is the highest level layer in the ZigBee network and application objects. The application object is developed to customize devices for various applications. It also controls and manages the protocol layer in a ZigBee device. The application layer consists of three parts: the application sub layer (APS), the application framework (AF), and the endpoints. 
- The application sub-layer: This layer interfaces the ZigBee application layer to the ZigBee networking layer. It also provides a common set of data transport services to all of the endpoints. The endpoints are what most people associate with ZigBee.

- The application frame (AF): This is the multiplexes and container that host application objects on a ZigBee device (endpoints). All endpoints are associated with application frames. The application frame gathers data that comes into application layer and sends them into their right destinations, the endpoint associated with the frame.

- The application object (endpoints): Endpoints are the numbers (up to 240 endpoints) of container spaces that stores all of application objects. The application object is basically a device profile with whatever extra functionality the user decides to add.

- ZigBee device object: This is a protocol within the ZigBee protocol stack that is responsible for overall device management and security keys and policies.

\subsubsection{Data Transfer to a Coordinator}

There are two methods that data can transfer to a coordinator in IEEE 802.15.4: beacon-enabled and nonbeacon-enabled. Figure 3.6 is shown the data transfer to coordinator in IEEE 802.15.4. The two approaches of transferring these data to the coordinator are shown in Figure 3.7. 


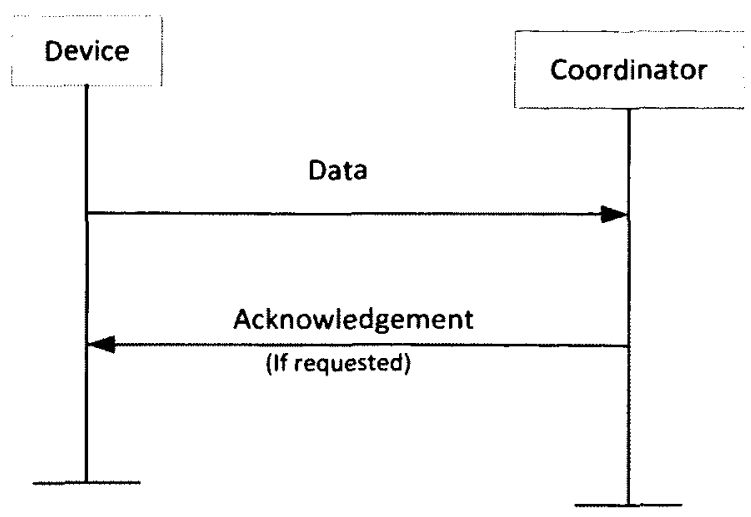

Figure 3.6: Data transfer to a coordinator in IEEE 802.15.4

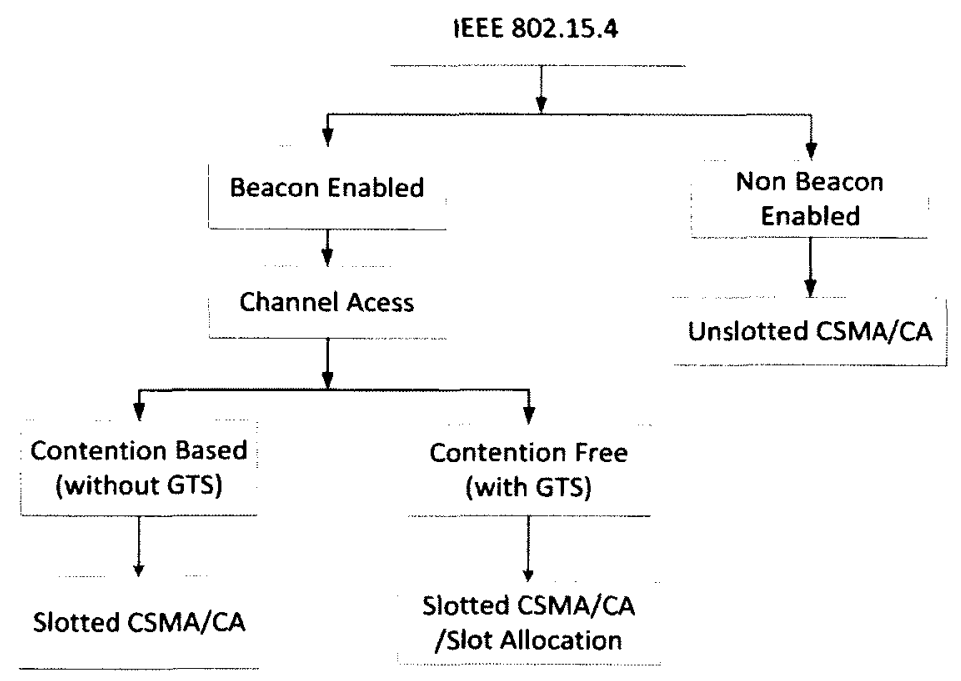

Figure 3.7: IEEE 802.15.4 functioning mode

\subsubsection{Beacon-enabled}

If the PAN coordinator selects the beacon-enabled mode to transmit the signal, there are two ways of transmitting the data: contention-based or contention-free. 
In contention-based, IEEE 802.15.4 (PAN coordinator) has implemented Carrier Sense Multiple Access with Collision Avoidance (CSMA-CA) method to allow all devices in the network to transmit the signal at the same frequency channel. There are two types of the CSMA-CA mechanisms in describe IEEE 802.15.4: CSMA-CA with slotted and CSMA-CA without slotted. In both circumstances, the algorithm is applied using units of time called back-off periods. When any device decides to transmit a signal, it must first perform clear channel assessment (CCA) to ensure that the desired channel is not in use by any other device.

In contention-free, IEEE 802.15.4 (PAN coordinator) allocates a time slot to a specific device. Without using CSMA-CA mechanism, the device with an allocated guaranteed time slot (GTS) with initially transmits the signal during GTS.

In a beacon-enabled network, the device continuously performs actively and requires a robust battery supply. Beacon-enabled also allows for heterogeneous networks. Some devices are active at all times, while others respond to stimulus and spend most of their time sleeping.

\subsubsection{Non Beacon-enabled}

If the PAN coordinator selects the non-beacon enabled mode, there are no channels to access and non-beaconenabled the device to use to transmit the data to the PAN coordinator. The device does not use slotted CSMACA mechanism to provide medium access control. One of the advantages of the non-beacon enabled network is that the ZigBee device spends much time sleeping, lowering their duty cycle (activity) and extending the battery life, as a result. The devices are active when the beacon transmits data, receives acknowledgement, and returns to sleep mode. 


\subsubsection{Data Verification Method}

Data verification method in IEEE 802.15.4 uses a 16-bit frame check sequence (FCS) based on the international Telecommunication Union (ITU) cyclic redundancy check (CRC) to detect possible errors in the data packet [58].

\subsection{Overview of the Research Prototype System}

In our research, we designed, developed, and implemented a wireless system consisting of several sensors to be used to monitor various Activities of daily living of a user by analyzing different changes in the environmental conditions in a smart home, collect information using microcontroller PIC, and wirelessly transmit the information to a base station using XBee modules. The collected information can be analyzed and displayed to a user's PC screen and simultaneously to a local liquid crystal display (LCD). A buzzer is also included in the prototype system to alert the user of any unacceptable changes in behaviour. The prototype system is costeffective, has low power consumption, and provides for wireless mesh networking. The prototype system can collect multiple data at the same time. The prototype system is divided into two parts: a remote station and a base station, as shown in the generic diagram of the prototype system in Figure 3.8 . 


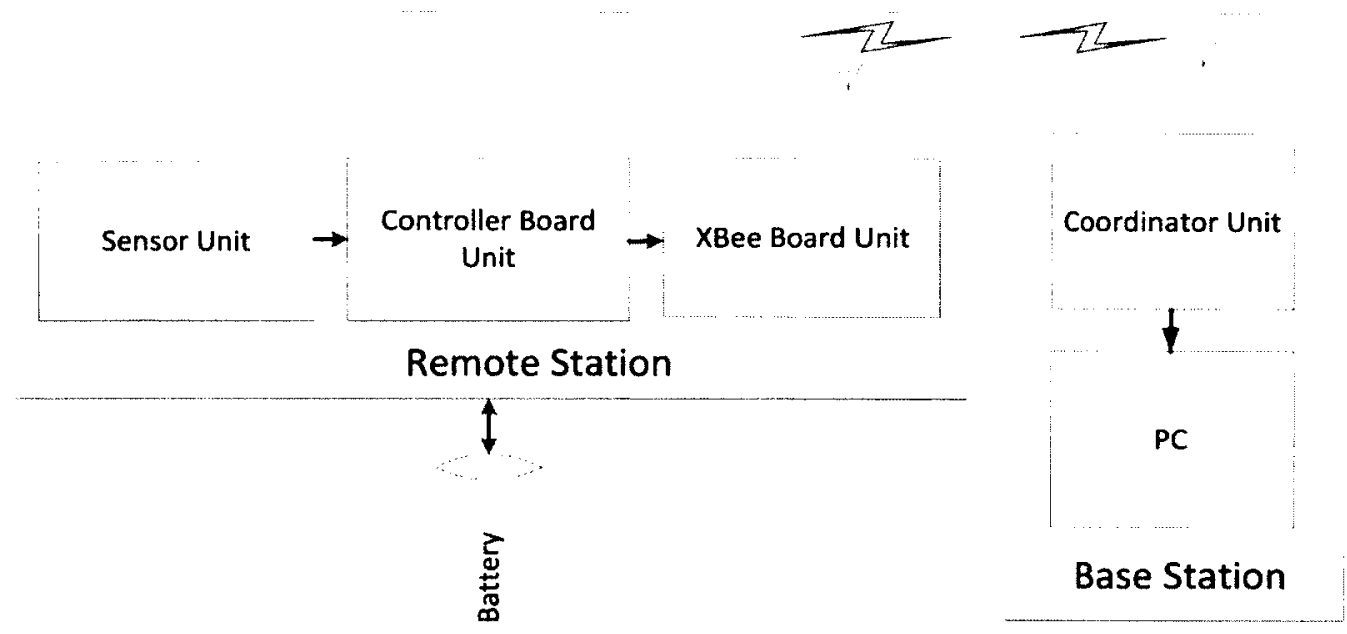

Figure 3.8: Generic figure of base station and remote station of the prototype system

- The remote station is composed of:

- A sensor unit,

- A controller board unit,

- An XBee module board unit, and

- Power by an energy source (usually a battery or solar energy)

- The base station consists of a coordinator unit connected to a PC.

The prototype system is made up of a set of independent remote stations, the base station as a gateway, and software. The remote station is a self-contained system consisting of several units and powered by an energy source, such as a battery or solar cell. The sensor unit consists of several sensors connected to the controller 
board unit. The controller board unit consists of a microcontroller PIC 16F876. The main functionality of the microcontroller in the research is to:

- Obtain raw samples of sensor inputs either in analogue or digital form;

- Convert these analog samples to digital binary; and

- Get ASCII code and compile information to be displayed to either on X - CTU terminal or LCD display.

An XBee board unit is an XBee module. Its main functionality is to wirelessly transmit acquired data to the gateway. The gateway is connected to the PC, which is used for collecting, processing, analyzing, and presenting the measurement data using X-CTU software. The software running on the gateway performs the appropriate computation and displays the information on the user screen.

\subsection{Components of the Research Prototype System}

In this section, we provide the description of each component of the prototype system in more detail. Figure 3.9 illustrates each component in the prototype system. As mentioned above, the prototype system is divided into two parts: a remote station and a base station. The remote station consists of several components, including sensors, a buzzer, a microcontroller, an XBee module, and a power source. 


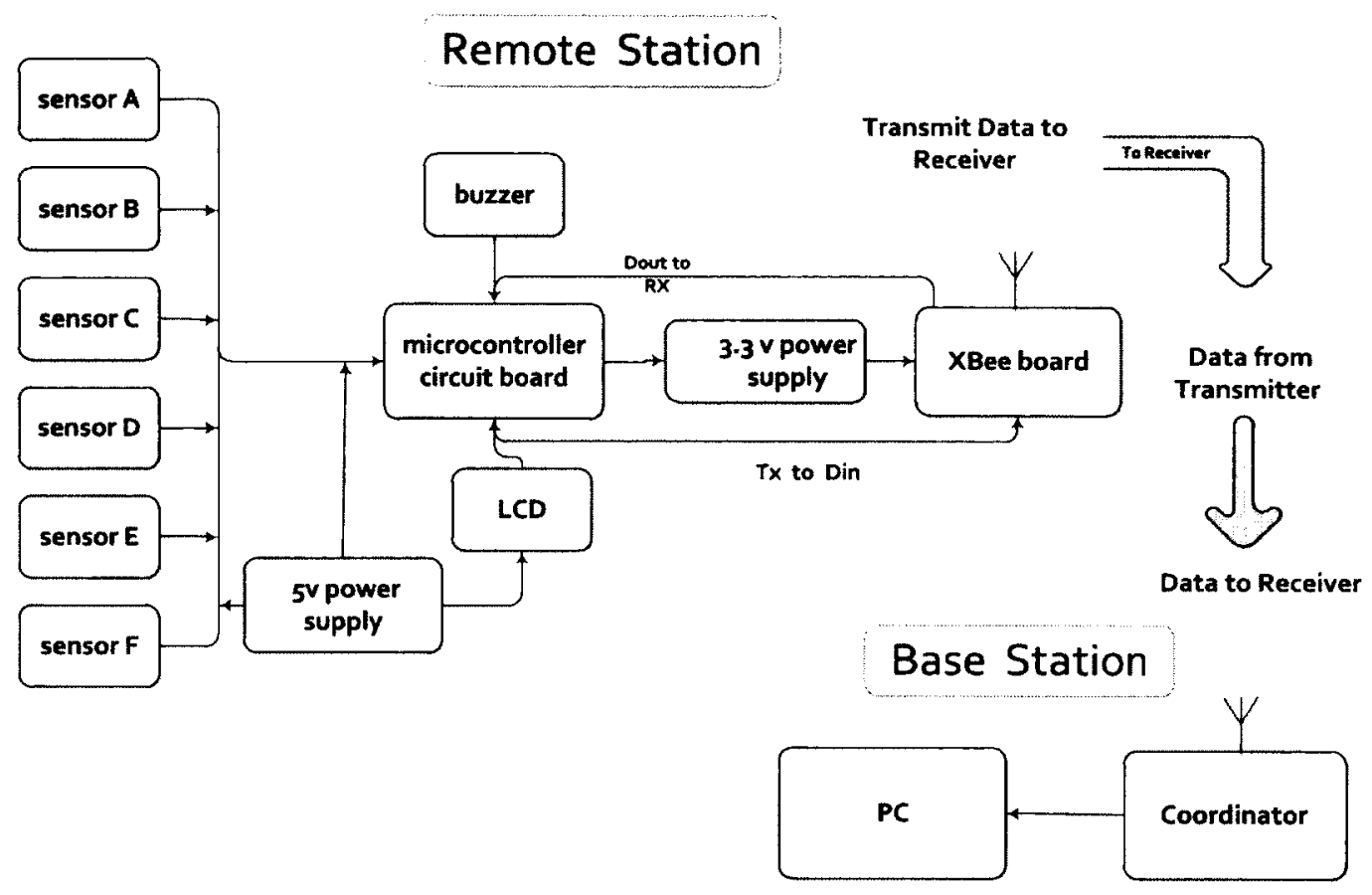

Figure 3.9: Functional block diagram of the system

The base station consists of a coordinator unit. The description of the prototype system is categorized into the following five parts:

(1) Sensors and buzzer;

(2) Microcontroller;

(3) XBee module;

(4) Power supply; and

(5) Coordinator. 


\subsection{Remote Station Hardware and Specification}

The remote station is composed of several sensors, a microcontroller board, an XBee Board, and a power source board. Six non-invasive sensors are composed of temperature, humidity, photocell, ultrasonic, pyroelectric ("passive") Infrared (PIR), Hall Effect, a $5 \mathrm{~V}$ regulator, and a piezoelectronic buzzer. The microcontroller board contains a microcontroller (PIC 16F876) and XBee Board is composed of an XBee module. Table 3.3 provides a complete list of the components we used for the purpose of this research and their cost.

\begin{tabular}{|c|c|c|c|c|}
\hline Name & Component Image & Description & Manubcturer & $\begin{array}{c}\text { Cost } \\
\text { (US Dollar) } \\
\end{array}$ \\
\hline XEee Module & & $\begin{array}{l}\text { XBee } 1 \mathrm{~mW} \text { Wire } \\
\text { Antenna-5eries } 1\end{array}$ & Diei international & 22.95 \\
\hline XBee Adapter & & $\begin{array}{c}\text { XBee Adapter kit - } \\
\text { vi.1 }\end{array}$ & Adufruit industries & 10.00 \\
\hline PKC1G:87G-2OV'SP & & $\begin{array}{l}\text { Microcontrollers } \\
\text { (MCU) } 14 \mathrm{~KB} 368 \\
\text { RAM } 221 / 0\end{array}$ & Microchip Tectinobgy & 7.84 \\
\hline 100 & & $\begin{array}{l}16 \times 2 \text { LCD Character } \\
\text { Display Modules }\end{array}$ & Lumex & 6.97 \\
\hline $\begin{array}{l}\text { Temperature } \\
\text { Sensor }\end{array}$ & & $\begin{array}{c}\text { TMP36- } \\
\text { Temperature Sensor }\end{array}$ & Analog Device Inx. & 1.50 \\
\hline Ultrasonic sensor & & $\begin{array}{c}\text { Maxbotix Lhrasonic } \\
\text { Rangefinda - L } L \text { - } \\
\text { EZ1 - EZ-1 }\end{array}$ & MaxBosix me. & 25.00 \\
\hline Humidity sensor & IIII & $\begin{array}{l}\text { HUMIOHTY SENSOR } \\
\text { 5V 5\% 3-PIN-CHS- } \\
\text { MSS-S Ersors } \\
\text { Trarsducers }\end{array}$ & TOK & 22.62 \\
\hline Light sensor & & $\begin{array}{c}\text { Photo cell } \\
\text { ICAS phota es stor! }\end{array}$ & Advanced Photonix he. & 1.00 \\
\hline Hall-Effect sensor & & $\begin{array}{l}\text { Sensor, Bipolar Hall } \\
\text { Effect, Radio Le ad IC } \\
\text { Padkege }\end{array}$ & Honerwell & 0.99 \\
\hline $\begin{array}{l}\text { P1R (motion) } \\
\text { sersor }\end{array}$ & & $\begin{array}{c}\text { Proelectrie } \\
\text { ("Passive")!nfraRed } \\
\text { sensors }\end{array}$ & Adsfuit shop & 10.00 \\
\hline Buzzer & & $\begin{array}{c}\text { Piezoelectronic } \\
\text { Buzer }\end{array}$ & TOK & 1.50 \\
\hline \multicolumn{4}{|c|}{ Total Cost } & 110.37 \\
\hline
\end{tabular}

Table 3.3: Prototype components used for the study and their cost. 


\subsection{Hardware and Specifications}

The features which are elaborated in the following sections include sensors and buzzers, a microcontroller board, an XBee module, and a $5 \mathrm{~V}$ power supply to the coordinator at the base station. Table 3.4 illustrates a list of the features of the components of the prototype system.

\begin{tabular}{|c|c|c|c|c|c|}
\hline \multirow[t]{2}{*}{ Components } & \multicolumn{5}{|c|}{ Features } \\
\hline & Type & Objective & Range & $\begin{array}{l}\text { Voltage } \\
\text { (V) }\end{array}$ & Manufacturer \\
\hline Microcontroller & PIC $16 F 876$ & $\begin{array}{l}\text { Capture } \\
\text { values }\end{array}$ & $-40^{\circ} \mathrm{C} \rightarrow 85^{\circ} \mathrm{C}$ & $2-5.5$ & Microchip \\
\hline $\begin{array}{c}\text { Temperature } \\
\text { Sensor }\end{array}$ & TMP36 & $\begin{array}{c}\text { Monitor } \\
\text { temperature }\end{array}$ & $-40^{\circ} \mathrm{C} \rightarrow 150^{\circ} \mathrm{C}$ & $2.7-5.5$ & Analog Devices \\
\hline Humidity Sensor & CHS- series & $\begin{array}{l}\text { Monitor } \\
\text { humidity }\end{array}$ & $5 \% \rightarrow 95 \%$ & 5 & TDK \\
\hline Light Sensor & PDV-P8001 & Detect light & --- & $\begin{array}{c}2.5- \\
\text { higher }\end{array}$ & $\begin{array}{c}\text { Advanced } \\
\text { Photonic, Inc. }\end{array}$ \\
\hline Ultrasonic Sensor & $\begin{array}{c}\text { LV-maxBotix- } \\
\text { EZO }\end{array}$ & $\begin{array}{l}\text { Detect } \\
\text { distance }\end{array}$ & $6 \rightarrow 254$ inch & $2.5-5.5$ & MaxBotix \\
\hline Motion Sensor & $\# 555-28027$ & $\begin{array}{l}\text { Detect } \\
\text { motion }\end{array}$ & $\begin{array}{c}20 \mathrm{ft} \\
\left(110^{\circ} \times 70^{\circ}\right)\end{array}$ & $3.3-5$ & Adafruit \\
\hline Magnetic Sensor & US5881LUA & $\begin{array}{c}\text { Detect } \\
\text { open/close } \\
\text { door }\end{array}$ & -- & $3.5-24$ & Melexis \\
\hline
\end{tabular}

Table 3.4: Prototype components used for the research

\subsubsection{Asynchronous Serial Communication}

It is important that different devices communicate with each other whether they are present on the same board or the same device or are on separate individual devices; thus, this communication has to be hardware selfdetermining. It should not matter what the devices or components are made of; however, there has to be a protocol for communication. There are large numbers of communication protocols available for devices to 
communicate with each other. The preferable communication system must synchronize its data transmission and receive a clock signal. In certain situations, such as in radio controlled wireless applications, it is difficult or sometimes impossible to establish a separate channel for data and a clock. In these situations, single wire transmission is more effective [59]. USART is the type of serial communication protocol that can establish this kind of transmission.

\subsubsection{Universal Asynchronous Receiver and Transmitter (USART) Protocol}

This protocol has two terminals: one terminal for receiving data and another for transmitting data. The data is sent and received with no need for clock synchronization. Therefore, it is called asynchronous. The USART protocol is very simple; it requires data composed of either 8 or 9 bits. Furthermore, The USART protocol can be configured as a full duplex asynchronous system that can communicate with peripheral devices, such as cathode-ray tube (CRT) terminals and personal computers, or it can be configured as a half-duplex synchronous system that can communicate with peripheral devices, such as analog-to-digital (A/D), digital-to-analog (D/A) integrated circuits, or serial EEPROM. USART is very commonly used in microcontrollers, because of which most of the microcontrollers provide a built-in hardware module such that this can be enabled. The hardware module has built-in circuitry to handle most of these USART tasks automatically [59], [60]. Microcontroller PIC 16 F876 has two external pins (RC6 and RC7) that are necessary to implement the USART protocol, RC6 for the data transmitter (Tx), and $R C 7$ for the data receiver $(R x)$. 


\subsection{ZigBee Starter Development Kits}

The ZigBee alliance is composed of several associated companies. As of 2007, there were over 200 semiconductor companies that have developed their own ZigBee protocol compatible hardware platforms and corresponding software stack packages, including MICAz from Crossbow, JN5139 from Jennic, CC2430 from Texas Instrument (Chipcon), and XBee from Digi International. In terms of cost, unrestricted development environment, flexibility, and management of advanced nodes with many peripherals, the type of ZigBee that we believe is suitable for this thesis at this stage is XBee, as shown in Table 3.5. 


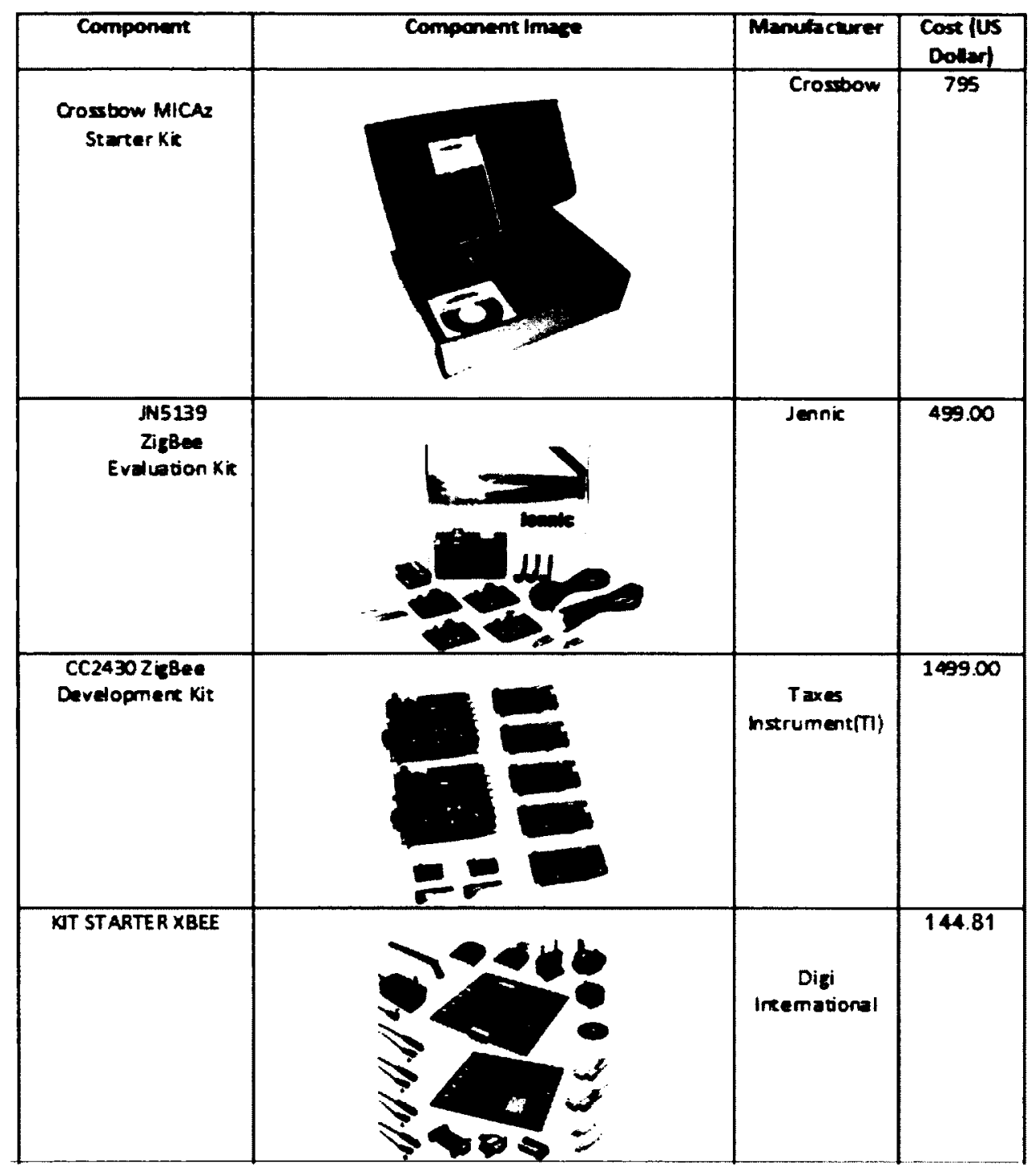

Table 3.5: ZigBee starter Kits

\subsection{XBee Module}

The brand of ZigBee module that we used for the research is called XBee. An XBee module is a wireless communication module developed by Digim International to meet IEEE 802.15 .4 standards that supports the 
unique needs of low-cost and low-power wireless sensor networks. The module is relatively small, reliable, and easy to use; requires minimal power; and provides consistently good quality and performance on the delivery of data between devices. The XBee module consists of a microcontroller and a radio frequency integrated circuit (RF IC). The module supports a wide range of communications protocols, such as 802.15.4 and Wi-Fi. The XBee module operates within the Industrial, Scientific, and Medical $(\mathrm{ISM})^{6}$ radio band frequency of $868 \mathrm{MHz}$ in Europe, $915 \mathrm{MHz}$ in the North America and Australia, and $2.4 \mathrm{GHz}$ worldwide.

\subsubsection{XBee Series 1 and XBee Series 2}

In our study, we began working with the XBee Series 1 module and then we switched to the XBee Series 2 module. XBee Series 1 and XBee Series 2 are not compatible. They use different chipsets, firmware, and protocol. They cannot be used together at the same time. In addition, XBee Series 1 is designed for point-topoint or point-to-multipoint tasks, while XBee Series 2 is suitable for mesh networks. Figure 3.10 shows an XBee Series 1 with RPSMA connector antenna.
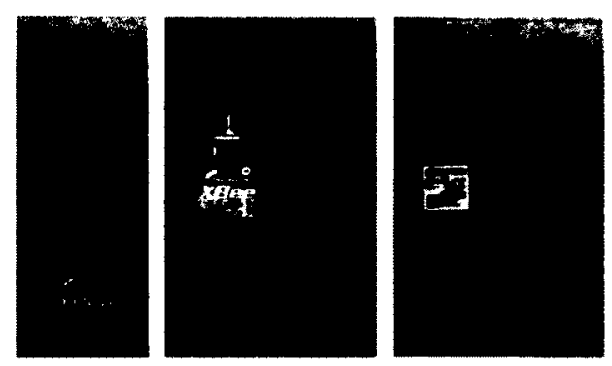

Figure 3.10: XBee Series 1 with RPSMA connector antenna

\footnotetext{
${ }^{6}$ ISM Band is the frequency band that is set aside to be used for scientific, medical, and industrial purposes.
} 
XBee Series 1 consumes less power compared to other versions of XBee. The typical range for the basic module is up to 100 feet ( 30 meters) in a normal home or office and 300 feet (100 meters) outside under Line-of-sight conditions.

On the other hand, XBee Series 2 has higher power consumption compared to the XBee Series 1 module. We switched from XBee Series 1 to Series $2^{7}$ because Series 2 has a higher range compared to Series 1 , and also Series 2 is suitable for mesh networking. Series 2 is capable of transmitting data to a maximum range of 133 feet (40 meters) indoors and 400 feet (120 meters) outdoors under Line-of-sight conditions. Series 2 can be separated into two sub-series: Znet 2.5 and ZB. The difference between these two series is that Znet can update firmware over-the-air while ZB Series 2 and Series 1 can only upgrade firmware with X-CTU via a serial communication port [61]. The specification of XBee Series 1 and Series 2 are illustrated in Table 3.6.

The XBee Series 2 modules that we used for the study are equipped with a $1 / 4$ wave monopole integrated whip antenna shown in Figure 3.11. The Series 2 module is based on the Ember ${ }^{8}$ chipset and is designed to be used in applications that require repeaters or mesh networking. Appendix $C$ provides more information on $X B e e$ modules.

\footnotetext{
${ }^{7}$ Series 2 consumes power approximately around $2 \mathrm{~mW}(+3 \mathrm{dBm})$.

${ }^{8}$ Ember is the ZigBee chip family that contains the highest wireless networking performance and application code space within the lowest power-consuming chip set.
} 


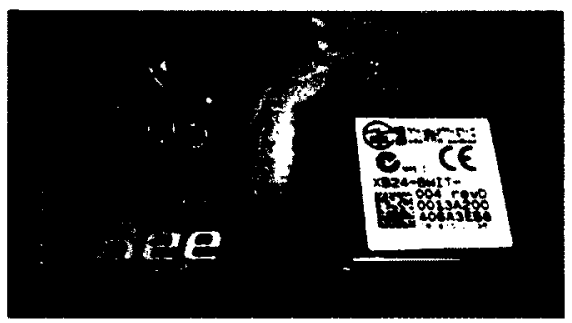

Figure 3.11: XBee Series 2 module with an integrated whip antenna

\begin{tabular}{|c|c|c|}
\hline & XBee Series 1 & XBee Series 2 \\
\hline Indoor/Urban range & up to $100 \mathrm{ft} .(30 \mathrm{~m})$ & up to $133 \mathrm{ft} .(40 \mathrm{~m})$ \\
\hline Outdoor $R F$ line-of-sight range & up to $300 \mathrm{ft} .(100 \mathrm{~m})$ & up to $400 \mathrm{ft} .(120 \mathrm{~m})$ \\
\hline Transmit Power Output & $1 \mathrm{~mW}(0 \mathrm{dbm})$ & $2 m W(+3 d b m)$ \\
\hline RF Data Rate & $250 \mathrm{Kbps}$ & $250 \mathrm{Kbps}$ \\
\hline Receiver Sensitivity & $-92 \mathrm{dbm}(1 \%$ PER $)$ & $-98 \mathrm{dbm}(1 \%$ PER $)$ \\
\hline Supphy Voltage & $2.8 \cdot 3.4 \mathrm{~V}$ & $2.8 \cdot 3.6 \mathrm{~V}$ \\
\hline Transmit Current (typical) & $45 \mathrm{~mA}(@ 3.3 \mathrm{~V})$ & $40 \mathrm{~mA}(@ 3.3 \mathrm{~V})$ \\
\hline lolle/Receive Current (typical) & $50 \mathrm{~mA}(3.3 \mathrm{~V})$ & $40 \mathrm{~mA}(@ 3.3 \mathrm{~V})$ \\
\hline Power-down Current & $10 u A$ & 1 UA \\
\hline Frequency & ISM $2.4 \mathrm{GHz}$ & ISM $2.4 \mathrm{GHZ}$ \\
\hline Dimensions & $0.0960^{\prime \prime} \times 1.087 "$ & $0.0960^{\prime \prime} \times 1.087^{\prime \prime}$ \\
\hline Operating Temperature & -40 to $85 \mathrm{C}$ & -40 to $85 \mathrm{C}$ \\
\hline Antenna Options & Chip, Integrated Whip, U.FL & $\begin{array}{c}\text { Chip, Integrated Whip, U.FL, } \\
\text { RPSMA }\end{array}$ \\
\hline Network Topologies & Point to point, Star & Point to point, Star, Mesh \\
\hline Number of Channels & 16 Direct Sequence Channels & 16 Direct Sequence Channels \\
\hline Filtration Options & $\begin{array}{l}\text { PAN ID, Channel \& } \\
\text { Source/Destination }\end{array}$ & $\begin{array}{l}\text { PAN ID, Channel \& } \\
\text { Source/Destination }\end{array}$ \\
\hline
\end{tabular}

Table 3.6: The specification of XBee series 1 and series 2 


\subsubsection{XBee Development Board}

The XBee module cannot function by itself; it needs to be connected to a circuit board to transmit or receive data. There are several XBee development boards that were considered in this research, such as SparkFun XBee Explorer USB board from SparkFun, ${ }^{9}$ XBee adapter from Adafruit, and Digi development board from Digi International. However, after investigation, we choose the SparkFun XBee Explorer USB board for the base station and an XBee adapter for the remote station. Appendix $C$ provides more information on the Digi International board.

\subsubsection{The XBee Board}

The XBee wireless modem adapter seen in Figure 3.12 is the board that we used for the remote station. It is an excellent low-cost adapter board, costing only $\$ 10$ as shown in Table 3.7. The module is the easiest way to create a wireless point-to-point or mesh network. It has error correction and is configured with AT commands. There are multiple kinds of the unit and it can even be used to create a wireless serial link out of the box. The connection to a computer via USB can be done using an FTDI cable, which means that configuring or upgrading the adapter can be as simple as plugging in a cable. RESET and RX pins are connected into the XBee, passing through a level converter chip that brings the levels to $3.3 \mathrm{~V}$; however, voltage between 2.7 to $5.5 \mathrm{~V}$ can be suitable to communicate with the XBee.

As shown in Figure 3.12, the adapter is very simple, consisting of the following components on board:

\footnotetext{
${ }^{9}$ Sparkfun is a well-known online electronic vender that sells bits and pieces to make electronics projects possible, website: www.sparkfun.com
} 
- $3.3 \mathrm{~V}$ regulator to supply XBee with power, up to $250 \mathrm{~mA}$;

- $\quad 5 \mathrm{~V}$ regulator to be used to connect to circuitry, such as the one we are using, without risk of damage;

- Two LEDs, one for activity (RSSI) and the other for power (associate); and

- 10-pin $2 \mathrm{~mm}$ sockets, included to protect the modem and allow for easy exchange, upgrading, or recycling.

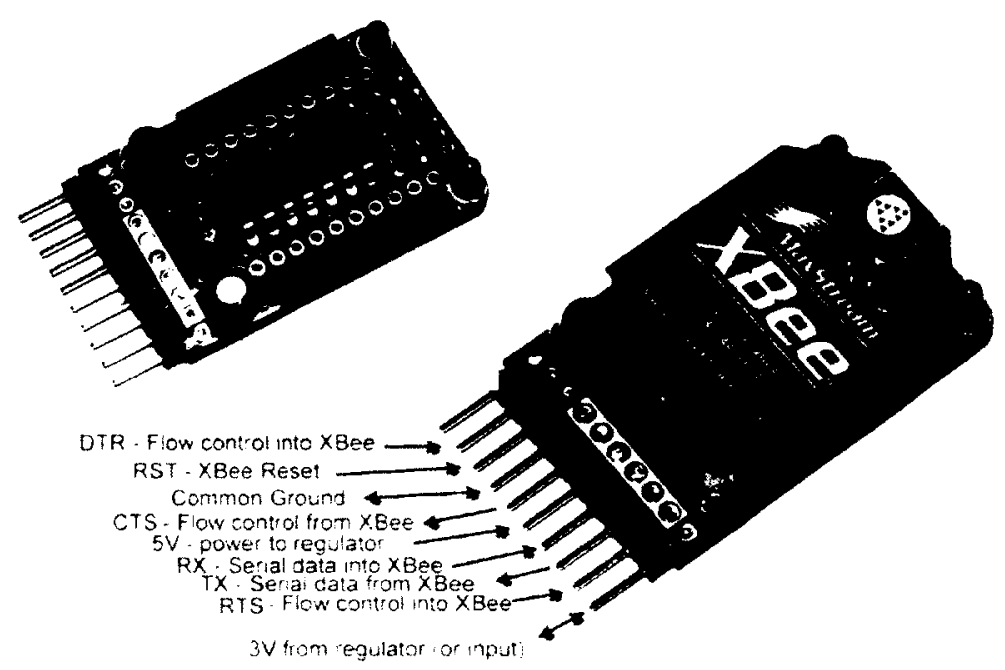

Figure 3.12: XBee Adapter ${ }^{10}$

\footnotetext{
${ }^{10}$ XBee Adapter from Adafruit, Adafruit website: $\underline{h t t p: / / w w w . a d a f r u i t . c o m ~}$
} 


\begin{tabular}{|c|c|c|c|}
\hline Component & Component Image & Manufacturer & Cost (US Doller) \\
\hline $\begin{array}{c}\text { Xeee Adapter kit - } \\
\text { v1.1 }\end{array}$ & & $\begin{array}{l}\text { Adafruit } \\
\text { Industries }\end{array}$ & 10.00 \\
\hline $\begin{array}{c}\text { FTDI Cable SV VCC. } \\
3.3 \mathrm{~V} \text { 1/0 }\end{array}$ & & $\begin{array}{l}\text { Adafruit } \\
\text { Industries }\end{array}$ & 20.00 \\
\hline \multicolumn{3}{|c|}{ Total } & 30.00 \\
\hline
\end{tabular}

Table 3.7: XBee adapter board

\subsection{Base Station - Receiver}

The base station consists of the coordinator unit and the PC. The coordinator unit is composed of an XBee module plugged into an XBee explorer USB board from SparkFun Electronics and connected to the PC through a USB Mini-B cable connector, as shown in Figure 3.13.

The main functionalities of the base station are the following:

- To collect, process, analyze, and present the measurement data using X-CTU software. The software running on the gateway performs the appropriate computation and displays the information on the user screen; the software is used for configuring the XBee modules as a coordinator for the base station or routers for the remote station. Further discussion of the X-CTU can be found in section 4.6.4. 
- To configure the XBee modules as a coordinator for the base station or routers and end devices for the remote station.

- The coordinator is used to establish the ZigBee network. Only one ZigBee coordinator and one or more end device are needed to form the personnel area network (PAN). The coordinator is configured to synchronize the communication network so that all wireless data received by the antenna are echoed through the USB port to the computer. The end device in the PAN has a unique PAN identity (known as PAN ID) to avoid miscommunication between PANs.

- The coordinator chooses the suitable channel with the appropriate PAN ID (16 and 64 bits) to establish the ZigBee network. The coordinator requests that the end device link to the network without knowing the address, PAN ID, or channel of the coordinator.

- The ZigBee coordinator is responsible for monitoring and controlling the overall network.

- The collected data are displayed on the X-CTU window, as shown in Figure 3.13.

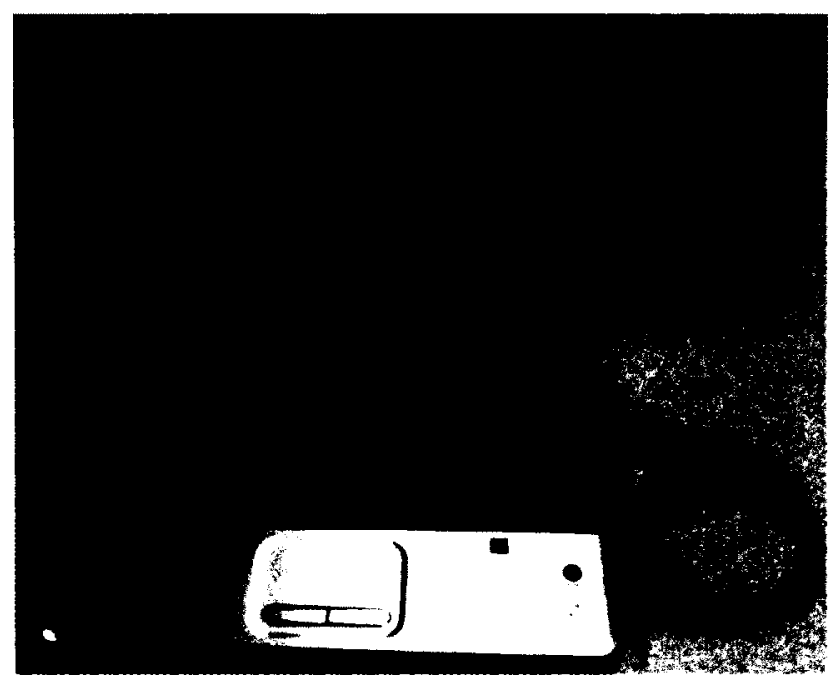

Figure 3.13: Base station of the prototype system 


\subsubsection{SparkFun XBee Explorer USB}

The XBee explorer USB board was selected for the base station. The board was chosen for the following reasons:

(1) It is reasonably small and cost effective;

(2) The board works well with almost all XBee modules, including Series 1, Series 2, Series 2.5, and XBee Pro;

(3) It is very simple to establish a connection with this board. All that is necessary is to plug the module into the XBee Explorer and attach it to a mini USB cable (as shown in Figure 3.14), which allows direct access to the serial and program pins on the XBee module.
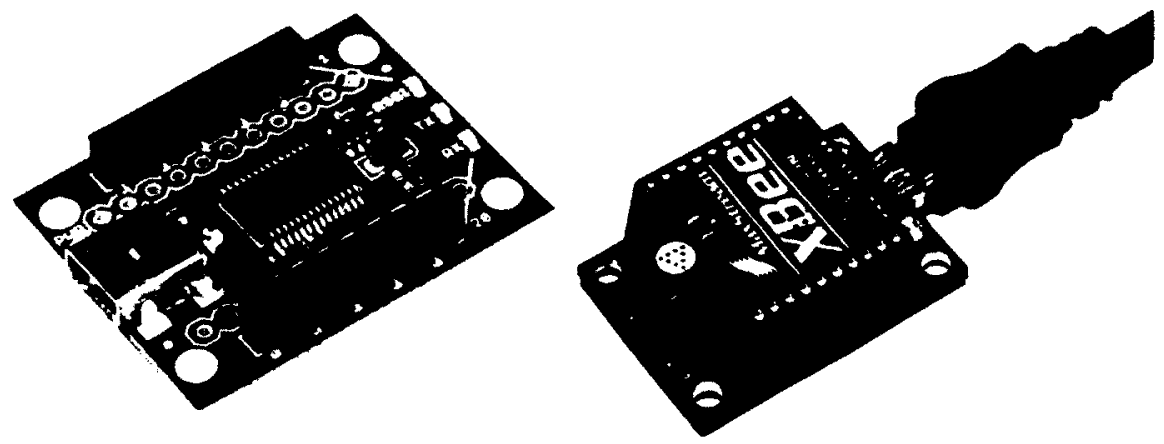

Figure 3.14: SparkFun XBee Explorer USB board ${ }^{11}$

Table 3.8 summarizes the cost of the XBee Explorer USB board.

\footnotetext{
${ }^{11}$ SparkFun XBee Explorer USB board from SparkFun, website: http://www.sparkfun.com/
} 


\begin{tabular}{|c|c|c|c|}
\hline Component & Component Image & Manufacturer & Cost (US Dollar) \\
\hline XBee Explorer USB & & $\begin{array}{l}\text { Sparkfun } \\
\text { Electronics }\end{array}$ & 24.95 \\
\hline USB Mini-B Cable & & $\begin{array}{l}\text { Sparkfun } \\
\text { Electronics }\end{array}$ & 3.95 \\
\hline \multicolumn{3}{|c|}{ Total } & 28.90 \\
\hline
\end{tabular}

Table 3.8: SparkFun XBee Explorer USB board 


\section{CHAPTER 4}

\section{DESCRIPTION OF THE HARDWARE AND SOFTWARE}

\subsection{Introduction}

The purpose of this chapter is to provide a detailed description of the hardware and software design of our prototype system. The chapter is divided into four sections: Section 4.2 shows the component images; section 4.3 provides information on the power supply for the prototype system. Section 4.4 contains an explanation of the connection of the components, and, finally, section 4.6 contains information on the software that we used to design and develop the prototype system.

\subsection{Component Images}

Figure 4.1 shows the actual images of the components we used for the research with arrows pointing to the direction of their connection. The figure consists of the following components:

Sensors: Temperature, Humidity, Light, Ultrasonic, Motion, and Hall Effect

Microcontroller: PIC $16 F 876$

XBee: Series 2

XBee Board

5V regulators: LM 7805

LCD and Buzzer 


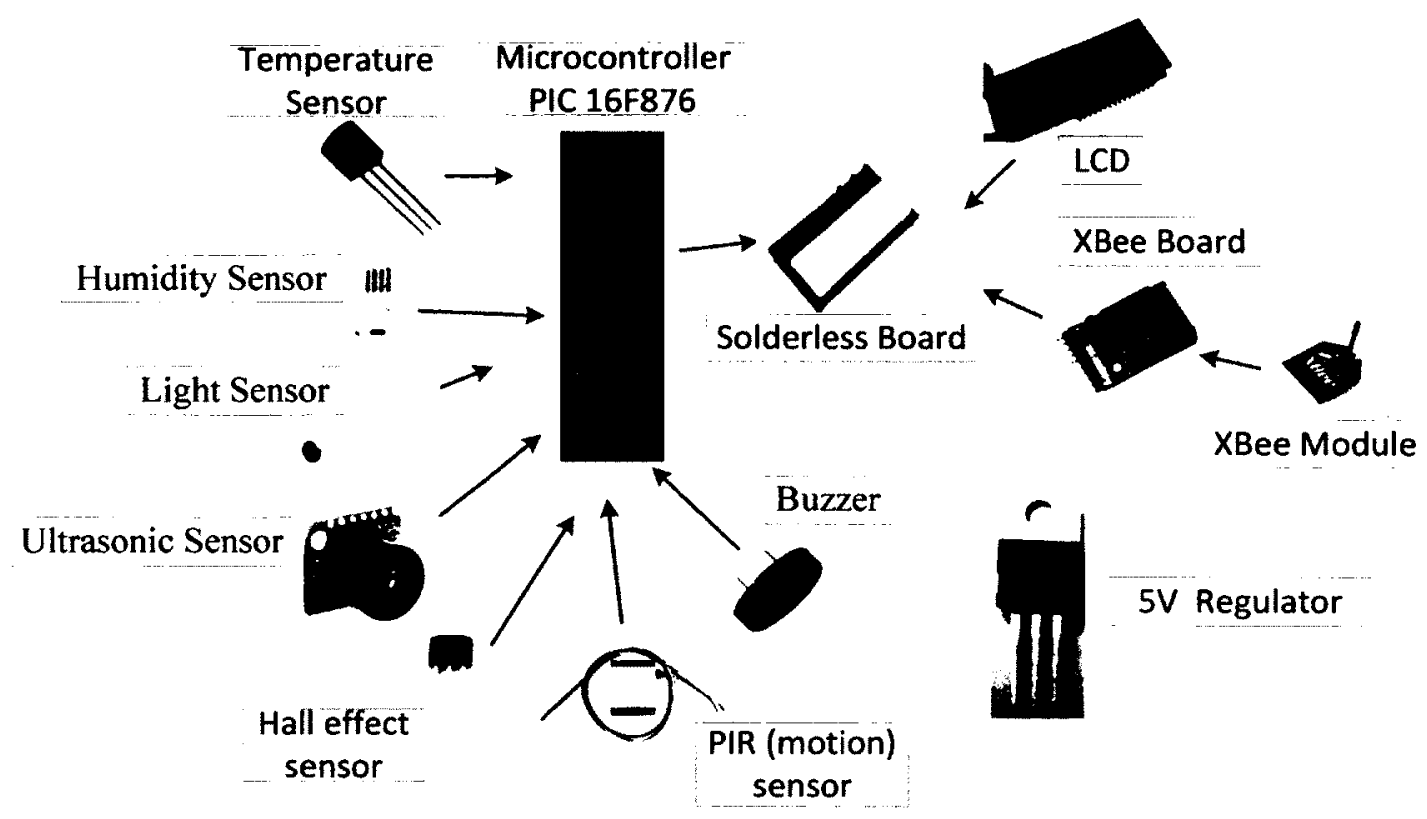

Figure 4.1: Developed remote station with all components

\subsection{V Power Supply Regulator}

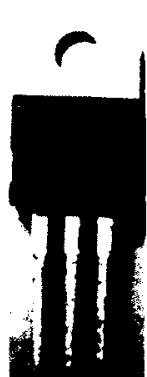

The first task is to design, develop, and implement a voltage regulator board to generate power to the microcontroller and other components. With the exception of the XBee module, which consumes only 3.3 V DC (Direct Current), the microcontroller PIC $16 F 876$ and other components require 5V DC power supply for their operation. The most common 5V power supply is LM7805. LM7805 has a power supply of $1 \mathrm{AMP}$ and a tolerance of $+/-5 \%$. In addition, a major concern for the accuracy of the output voltage is the $A C$ noise coming into the input pin. This noise must be reduced. The easiest way to help flatten out ripple is by using filtering capacitors. Electrolytic capacitors and ceramic capacitors were the two types of capacitors chosen for this task. These two types of capacitors and other components in Table 4.1 and Figure 4.2 were used to design the SV DC power supply for the prototype system. 


\begin{tabular}{|c|c|}
\hline Component & Voltage (V) \\
\hline XBee Series 1 & $2.8-3.4$ \\
\hline XBee Series 2 & $2.8-3.6$ \\
\hline $\begin{array}{c}\text { Microcontroller (PIC } \\
\text { 16F876) }\end{array}$ & $2-5.5$ \\
\hline liquid crystal display (LCD) & 5 \\
\hline Temperature Sensor & $2.7-5.5$ \\
\hline Humidity Sensor & 5 \\
\hline Light Sensor & up to 100 \\
\hline Ultrasonic Sensor & $2.5-5.5$ \\
\hline PIR (Motion) sensors & $3.3-5$ \\
\hline Hall - Effect Sensor & $3.5-24$ \\
\hline Piezo Buzzer & $3-30$ \\
\hline
\end{tabular}

Table 4.1: Components and their voltage ranges

- Switch: Use to turn system ON/OFF contains three pins that can easily be snapped into a breadboard to turn the system on or off. The center pin is connected to the power. Sliding forward turns the system off and sliding backward turns the system on.

- DC jack: Terminal for power source to the system contains pins that snap into a breadboard.

- 1N4001 protection diode: Allows the current to flow in one direction, thus blocking the current from flowing in the opposite direction.

- Two electrolytic capacitors and two 0.1uF ceramic capacitors: Are used to stabilize the supply output and input.

- Two LEDs and matching resistors: The matching resistors are used to oppose an electric current by making a voltage drop between its terminals in proportion to the current.

From Ohm's law (Voltage $(V)=$ current $(I) \times$ Resistor $(R) \rightarrow V=I R$ ) with a $5 V$ power supply and a current of 0.005 , the matching resistor is $5 / 0.005=1000 \mathrm{Ohm}$. 


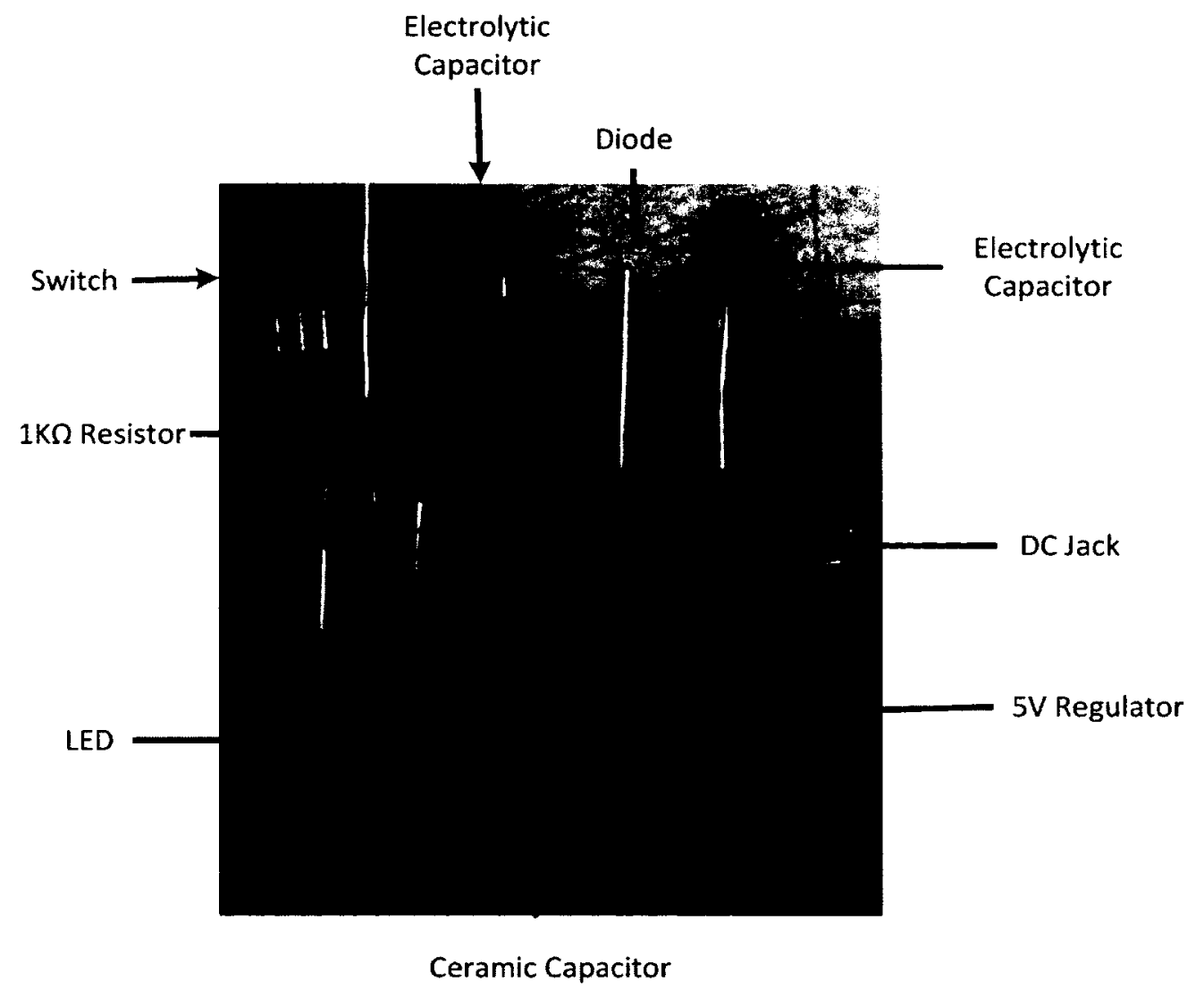

Figure 4.2: $5 \mathrm{~V}$ power components

Figure 4.3 illustrates the 5V DC power supply that was designed for the study. The figure on the right is when the power switch was turned on, and the figure on the left is when the power switch was turned off. We used a voltmeter to test the power output. Appendix D provides more information related to designing the $5 \mathrm{~V}$ power supply for the system. 
5V Regulator Circuit OFF

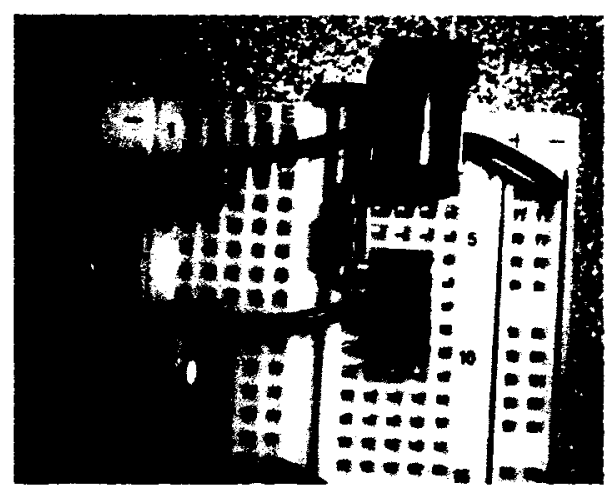

5 V Regulator Circuit ON

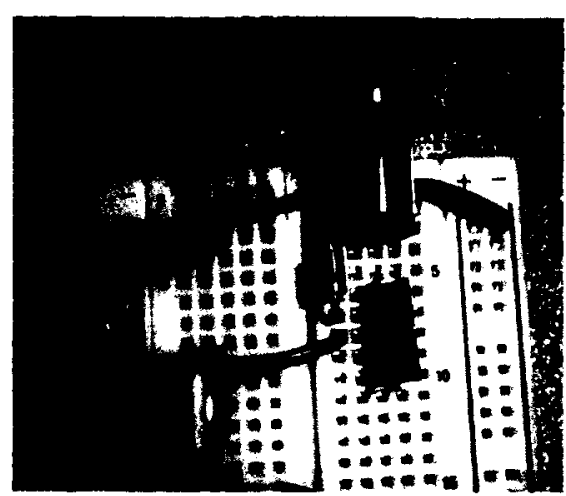

Figure 4.3: $5 \mathrm{~V}$ regulator circuit designed board when the switch is off and on

\subsection{Component Connections}

The components and the orders of their interconnections are shown in Figure 4.4. Once the 5V power supply is functioning properly, the next task is to design and develop the microcontroller board. The microcontroller is designed and developed for its functionality by using the LED, buzzer, and LCD, which are programmed to test for the following: LED to test if it's blinking, buzzer to check it's buzzing, and LCD to test if we could display output from it. Once the microcontroller is confirmed to be working well, the sensors are connected one by one and the display of their output on the LCD begins. Then, we connected the XBee module to transmit the collected data to the base station. The components and order of their connections in this system can be described in the four following steps:

(1) Step 1 - microcontroller, PIC 16 F876

(2) Step 2 - sensors, LCD, and piezoelectronic buzzer

(3) Step 3 - XBee module 
(4) Step 4 - coordinator

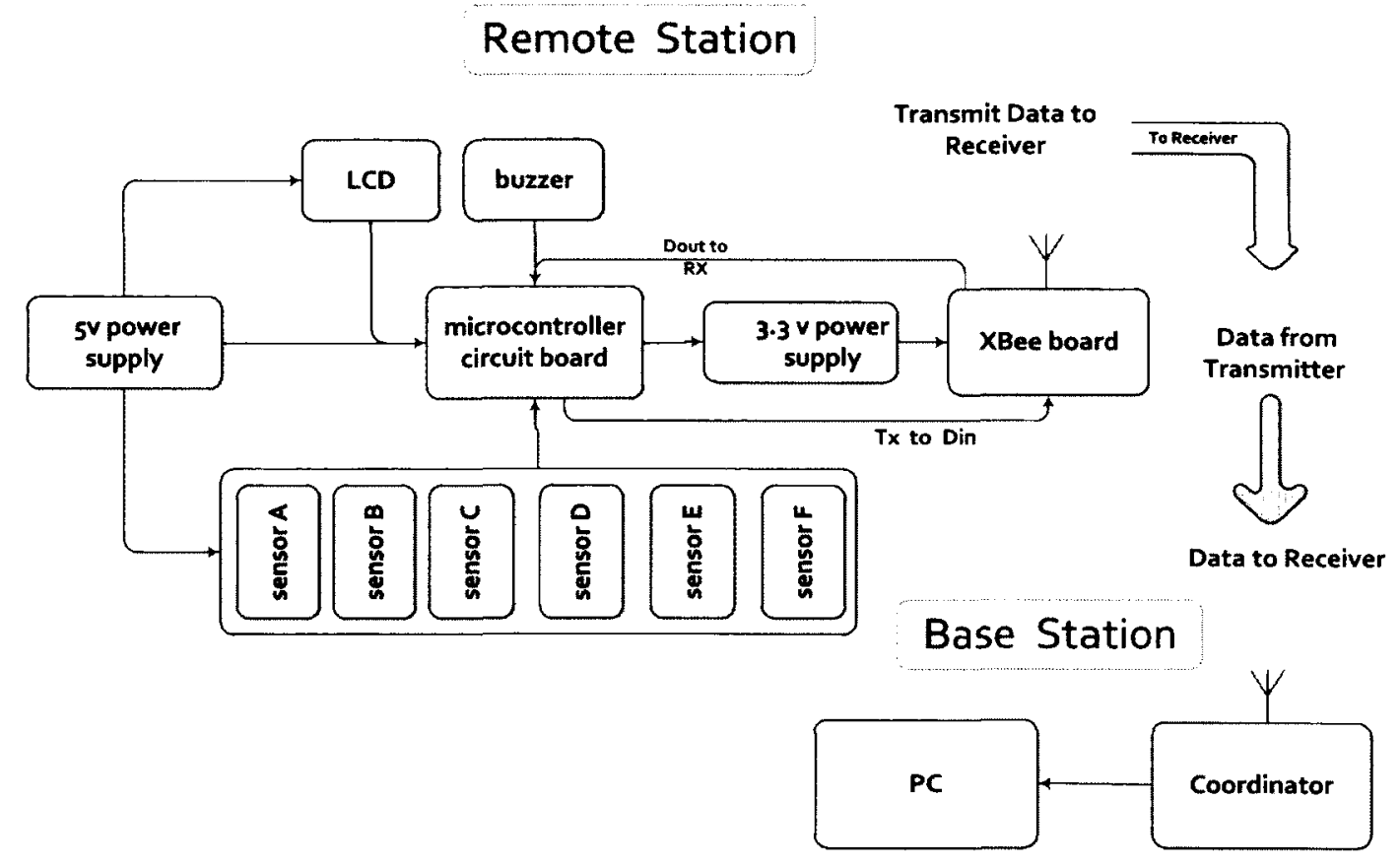

Figure 4.4: Block diagram of the prototype design

\subsection{Components Connection Pins}

Microcontroller PIC 16F876 is the heart of the remote station. All of the components on the remote station system must be connected to the microcontroller to be programmed to transmit data to the base station via the XBee modules. These components consist of the XBee modules, liquid crystal display (LCD), Hall Effect Sensor, and buzzer and monitor temperature, humidity, photocell, ultrasonic, and Pyroelectric ("passive") Infrared (PIR). 


\subsubsection{Microcontroller PIC 16F876 Pin Assignments}

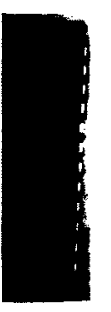

Microcontroller PIC 16F876 has three input and output (1/O) ports: PORTA, PORTB, and PORTC. These ports are bidirectional, which means the ports can be set as either inputs or outputs. These ports provide the means of communication with the microcontroller. PORTA has six pins, RAO (least significant bit) to RA5 (most significant bit), as shown in the Figure 4.5 and Table 4.2. PORTB and PORTC have eight pins each, RBO to RB7 and RCO to RC7, respectively. Both PORTB and PORTC are 8-bit ports. In this thesis, PORTB is devoted to LCD usage. The LCD is used to display data locally. RC 6 and RC 7 of PORT C are used as the UART PORT to transmit and receive the collected data. Table 4.2 shows the pins of the microcontroller assigned for the function of the components.

\begin{tabular}{|c|c|c|c|c|c|}
\hline IC Pin & $\begin{array}{c}\text { PORT A } \\
\text { Bit }\end{array}$ & IC Pin & PORT B Bit & IC Pin & PORT C Bit \\
\hline Pin 2 & RA0 & Pin 21 & RB0 & Pin 11 & RC0 \\
\hline Pin 3 & RA1 & Pin 22 & RB1 & Pin 12 & RC1 \\
\hline Pin 4 & RA2 & Pin 23 & RB2 & Pin 13 & RC2 \\
\hline Pin 5 & RA3 & Pin 24 & RB3 & Pin 14 & RC3 \\
\hline Pin 6 & RA4 & Pin 25 & RB4 & Pin 15 & RC4 \\
\hline Pin 7 & RA5 & Pin 26 & RB5 & Pin 16 & RC5 \\
\hline & & Pin 27 & RB6 & Pin 17 & RC6 \\
\hline & & Pin 28 & RB7 & Pin 18 & RC7 \\
\hline
\end{tabular}

Table 4.2: Structure of ports for microcontroller PIC $16 F 876$ 


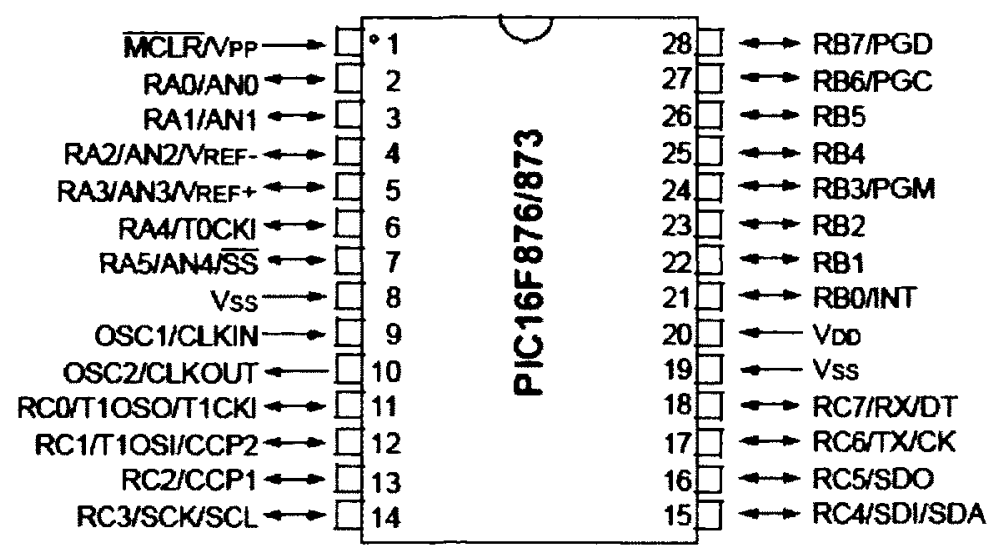

Figure 4.5: Microcontroller PIC 16 F876

Appendix B provides more information on the microcontrollers and other components that we considered for the purpose of this research.

\subsubsection{Analog to Digital Conversion}

Most sensors provide analog voltage readings that must be converted to a digital form using an $A / D$ converter.

The digital equivalent to the analog voltage can be determined from Equation 1.

$$
\text { output voltage }{ }^{12}=\left(\frac{\text { raw reading }}{2^{\text {ADC bit }}-1}\right) \times \text { refence } \operatorname{voltage}(v)
$$

Where:

Raw reading is an incoming raw reading that will contain any reading value from 0 to $2^{A D C}$ bit -1 .

Reference voltage is $5 \mathrm{~V}$ power supply; therefore, by default, the reference is set to $5 \mathrm{~V}$.

${ }^{12}$ Output voltage is converted into volt by $(5 / 1023) *$ raw reading. Raw reading contains a value from 0 to 1023. 
ADC Bit is the microcontroller PIC resolution. The microcontroller PIC recommends a 10-bit resolution of analog to digital conversion. This indicates that when measuring an arriving voltage, the PIC microcontroller matches up that voltage to a reference voltage and gives the associated representation number from 0 to 1024-1 $\left(2^{10 \text { bit resolution }}-1\right)$. Therefore, the returned raw reading value is converted into volts by $(5 / 1023)^{*}$ raw reading. 0 indicates the lowest measurement voltage and 1023 represents the highest measurable voltage. The output will be 0 when the analog input is $0 \mathrm{~V}$, and the output will be 1023 when the analog input is $5 \mathrm{~V}$, and each step indicates an increment of $5 \mathrm{~V} / 1023=0.00488$.

$$
\text { output voltage }=\left(\frac{\text { raw reading }}{1023}\right) \times 5 \mathrm{~V}
$$

To get voltage in $\mathrm{mV}$, we multiplied equation (2) by 1000 to get equation (3):

$$
\text { output voltage }=(\text { raw reading }) \times 4.88
$$

Equation (3) is used to obtain the digital values of the thesis components.

\subsubsection{Temperature Sensor}

The TMP36 temperature sensor output voltage is ((output voltage) $\mathrm{mV}-500)$ per 10 degrees centigrade $(10 \mathrm{mV} /$ centigrade). From equation (3), the temperature can be determined as follows:

$$
\text { temperature }\left(\mathrm{C}^{0}\right)=\left[\frac{\text { raw reading } 1 \times 4.88-500}{10}\right]
$$

Equation (4) can be reduced to equation (5):

$$
\text { temperature }\left(\mathrm{C}^{0}\right)=[((\text { raw reading } 1) \times 0.488)-50]
$$


For example, if raw reading1 is equal to number 185 , the corresponding temperature it will be

$$
\begin{aligned}
\text { temperature }\left(C^{0}\right) & =[((185) \times 0.488)-50] \\
\text { temperature }\left(C^{0}\right) & =[(90.28)-50] \\
\text { temperature }\left(C^{0}\right) & =40.28
\end{aligned}
$$

Figure 4.6 shows the voltage output in volts responding to the temperature in centigrade.

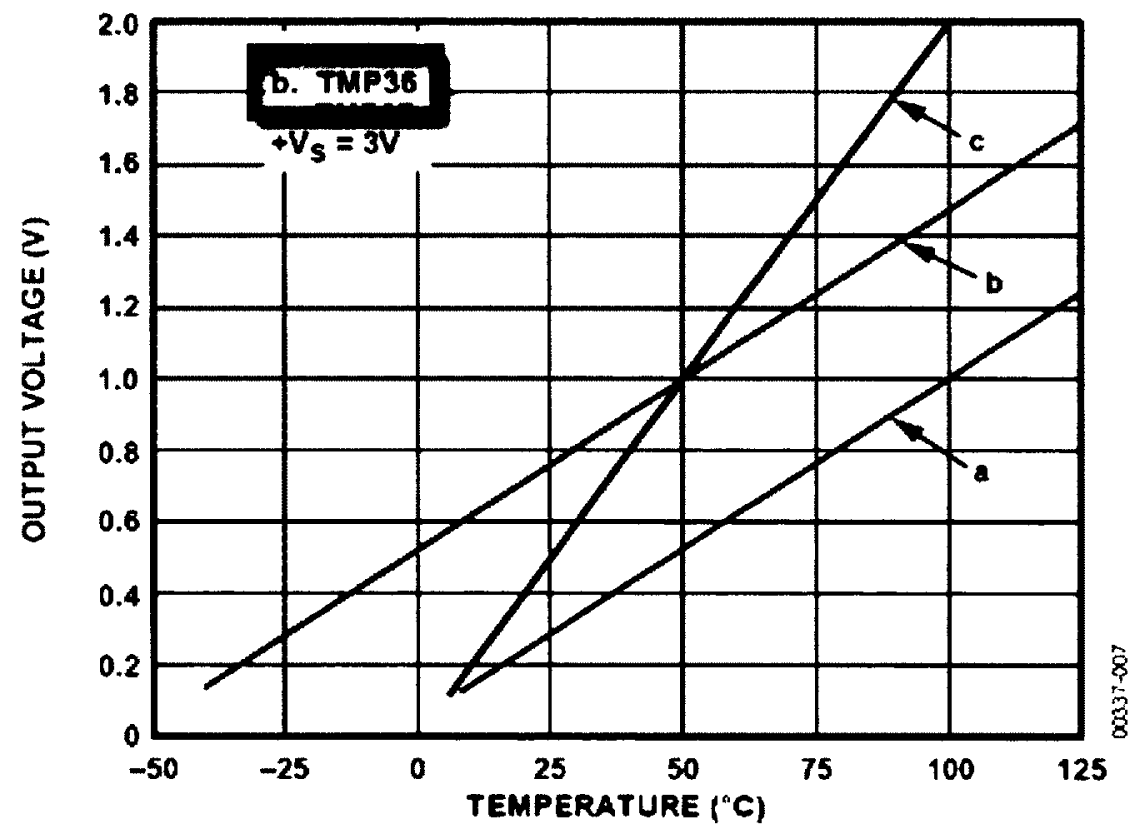

Figure 4.6: Output voltage (V) vs. Temperature $\left({ }^{0} \mathrm{C}\right)^{13}$

The TMP36 Temperature sensor is a three pin IC sensor. The sensor should be connected as follows: the left pin is connected to power (5V), the right pin is connected to a ground, and the middle pin (an analogy voltage that is directly proportional (linear) to the temperature) is connected to PIC16F876 at RAO of PORTA.

\footnotetext{
${ }^{13}$ Source of this figure: Adafruit, ladyada, http://www.ladyada.net/learn/sensors/tmp36.html
} 


\subsubsection{Ultrasonic Sensor}

Ultrasonic Sensor provides an output analog voltage with a scaling factor of $(\mathrm{Vcc} / 512)$ per inch,

with a power supply of $5 \mathrm{~V}$ yields $\sim 9.8 \mathrm{mV} / \mathrm{in}$. and $3.3 \mathrm{~V}$ yields $\sim 6.4 \mathrm{mV} / \mathrm{in}$.

From equation 2, the ultrasonic sensor reading can be determined as the following:

$$
\text { raw reading } 2=\left[\left(\frac{1023}{5 \mathrm{~V}}\right) \times 9.8 \mathrm{mV} / \text { distance }(\text { inch })\right]
$$

Equation (6) can be reduced to equation (7):

$$
\operatorname{distance}(\text { inch })=\left[\left(\frac{(\text { raw reading } 2)}{2.00508}\right)\right]
$$

The sensor has three pins: pin 3 (right) is connected to $5 \mathrm{~V}$ power. Pin 2 (middle) is connected to the ground; and pin 1 (left) is connected to the RA 1 of PORTA of the microcontroller.

\subsubsection{Humidity Sensor}

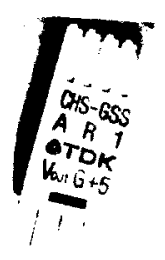

The relative humidity $(\mathrm{RH})$ can be read directly with a voltmeter; an output DC of $1 \mathrm{~V}$ can produce $100(\%) \mathrm{RH}$. The sensor is capable of measuring humidity in the range of $5 \%$ to $95 \%$ relative humidity. Relative humidity can be determined from equation (2) by multiplying equation (2) by 100 to get equation (8):

$$
\text { humidity }(\%)=\left[\left(\frac{(\text { raw reading } 3) \times 5 V}{1023}\right) \times 100\right]
$$

Equation (8) can be reduced to equation (9):

$$
\text { humidity }(\%)=(\text { raw reading } 3) \times 0.488
$$


The sensor was connected to RA 2 of PORT A of the microcontroller.

\subsubsection{Photo Cell Sensor (Light Sensor)}

$\overrightarrow{7}$

The photocell sensor has two pins. The pins are symmetric. One of the pins is connected to the $5 \mathrm{~V}$ power supply, and the other pin is connected to RA3 of PORTA of microcontroller PIC 16 F876.

The photocell's sensor can be determined from equation 2 above as follows:

$$
\text { light }=\left(\frac{\text { raw reading } 4}{1023}\right) \times 5 \mathrm{~V}
$$

Equation (10) can be reduced to equation (11):

$$
\text { light }=(\text { raw reading } 3) \times 0.00488
$$

\subsubsection{PIR (Motion) Infrared Sensor}

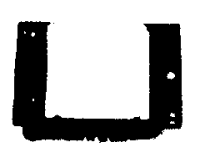

PIR (Motion) Infrared Sensor output provides a binary reading. The sensor has three pins. Pin 3

(right) is connected to $5 \mathrm{~V}$ power. Pin 2 (middle) is connected to the ground, and pin 1 (left) is connected to the RC 4 of PORTC of microcontroller. The motion is detected when the RC 4 of PORT C output is ' 1 ' and motion is not detected when the RC 4 of PORT C output is ' 0 '. 


\subsubsection{Hall Effect (magnetic) Sensor}

The Hall Effect (magnetic) Sensor has three pins, pin 3 (right) is connected to $5 \mathrm{~V}$ power, pin 2 (middle) is connected to the ground; and pin 1 (left) is connected to the RC3 of PORT C. The sensor operates with a magnet, as shown in Figure 4.6. When the south pole of the magnet is near the front of the sensor, pin 3 will go down to OV. In our research, this will indicate that the door is closed. Otherwise, it will stay at whatever the pullup resistor is connected to and display the door being unlocked on the screen. In the north pole of the magnet, nothing occurs if a magnet is placed nearby (unipolar).

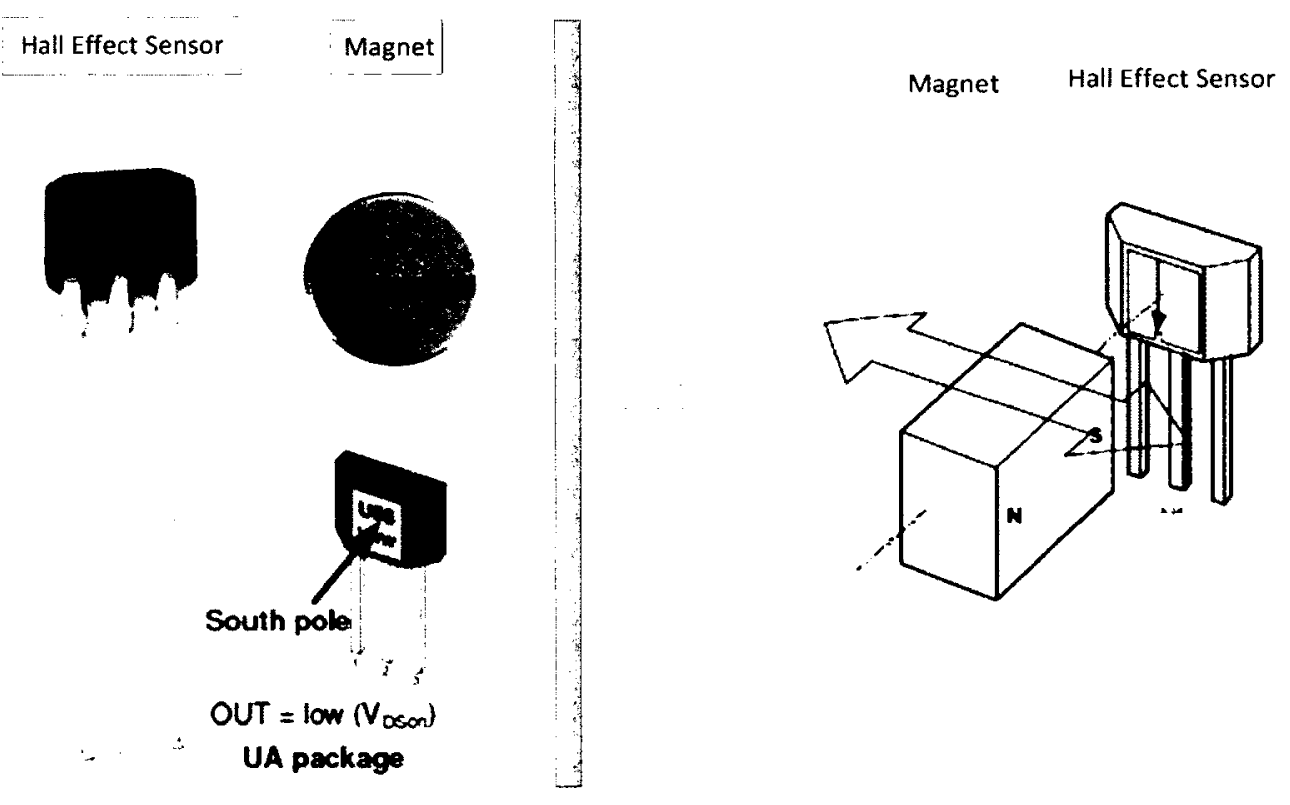

Figure 4.7: Hall Effect sensor with magnet

\subsubsection{Piezoelectric Buzzers}

Piezoelectric buzzers are used to alert any unacceptable changes in behaviour. It consists of two pins.

The pins are connected to RB2 of PORT B and ground of the microcontroller. 


\subsubsection{Liquid Crystal Display (LCD)}

A $16 \times 2$ backlight liquid crystal display (LCD) is the type of LCD that we used for this thesis. The LCD can display letters, numbers, and a few symbols. All character and letter fonts are developed into the hardware of the LCD device. The LCD is connected to a $5 \mathrm{~V}$ power supply; the ground; and RBO, RB1, and RB4 of PORT B. The goal of the LCD is to display the data results locally.

\subsubsection{XBee Module Communication Protocol}

The XBee module communicates with other devices through Universal Asynchronous Receiver and Transmitter (UART) protocol. The UART communication pins of the XBee module are connected directly to the microcontroller's UART, as illustrated in Figure 4.8. The XBee module needs two wires, one for transmitting the data and the other for receiving the data. In our case, these two wires are directly attached to RC6 and RC7 of PORT $C$ of the microcontroller to establish communication with other devices. For serial data, data enters the module UART through the DIN (pin 3) and exits the module UART through the DOUT (pin 2) as an asynchronous serial signal.

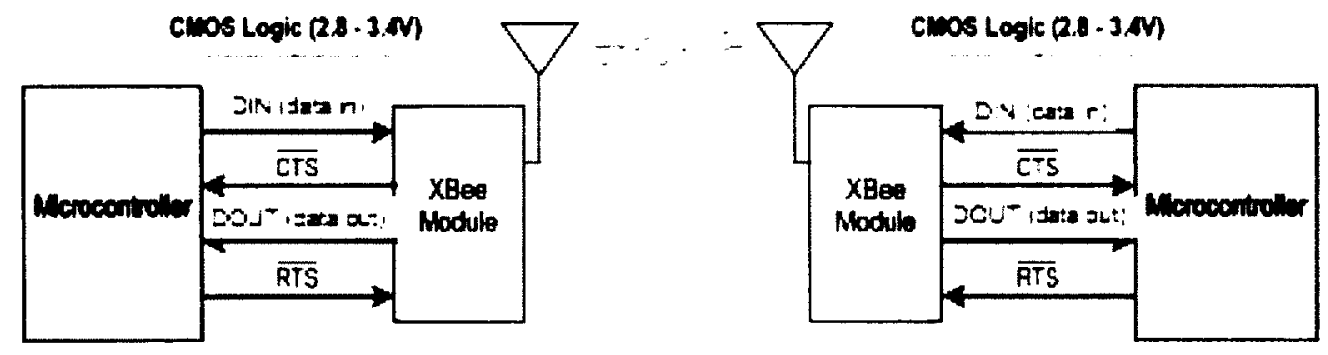

Figure 4.8: System data flow diagram in a UART-interfaced environment ${ }^{14}$

\footnotetext{
${ }^{14}$ Source Digi International: http://ftp1.digi.com/support/documentation/90000866 C.pdf
} 


\subsubsection{XBee Module Pins}

Table 4.3 shows pin assignments for the XBee and XBee-Pro modules with brief descriptions of all 20 pins in the modules; however, the pin numbers of significance for the study are the following five pins: pin $1, \operatorname{pin} 2$, pin 3 , pin 5, and pin 10.

\begin{tabular}{|c|c|c|c|}
\hline Pin & Name & Direction & Description \\
\hline$T$ & VCC & - & Power supply \\
\hline 2 & DOLT & Output & LART data out \\
\hline 3 & DIN: $\overline{\text { CONFIG }}$ & Input & UART data in \\
\hline 4 & DO8 & Output & Digital output 8 \\
\hline 5 & $\overline{\text { RESET }}$ & Input & Module reset (at least $200 \mathrm{nS}$ ) \\
\hline 6 & PWMI0 : RSSI & Output & PWM output $0: R X$ signal strength indicator \\
\hline 7 & PWM1 & Output & PWM output I \\
\hline 8 & (reserved) & & Do not connect \\
\hline 9 & 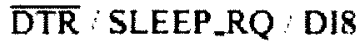 & Input & Pin sleep control line or digital input 8 \\
\hline 10 & GND & - & Ground \\
\hline 11 & $\mathrm{AD4}: \mathrm{DIO} 4$ & Either & Analog input 4 or digital 104 \\
\hline 12 & $\overline{\text { CTS }}$ DIO? & Either & Clear to send flow control or digital 107 \\
\hline 13 & ON $\overline{\text { SLEEP }}$ & Output & Module status indicator \\
\hline 14 & VREF & Input & Voltage reference for $A D$ inputs \\
\hline 15 & Associate AD5: DIOS & Either & Associated indicator, analog input 5 or digital I:O 5 \\
\hline 16 & $\overline{\mathrm{RTS}}$ AD6 : D1O6 & Either & RTS flow control, analog input 6 or digital 106 \\
\hline 17 & AD3 : DIO3 & Either & Analog input 3 or digital $1 \mathrm{O}_{3}$ \\
\hline 18 & AD2 $: \mathrm{DIO}_{2}$ & Either & Analog input 2 or digital 102 \\
\hline 19 & ADI:DIOI & Either & Analog input 1 or digital $\mathrm{I}: \mathrm{O}$ \\
\hline 20 & ADO DIOO & Either & Analog input 0 or digital $1: 0$ \\
\hline
\end{tabular}

Table 4.3: Pin assignment for the XBee and XBee PRO modules [61]

- Pin number $1, V c c$, is a power-supply pin; its practical use is to provide the XBee module with the power it needs to transmit or receive data

- Pin number 2, DOut, is a pin for "UART Data Out"; its purpose in the design of the module is to transmit data to the receiver

- Pin number 3, DIn, is a pin for "UART Data In"; its task is to receive data 
- Pin number 5 is an XBee reset pin

- Pin number $10, \mathrm{GND}$, is ground pin

\subsubsection{Remote Station Experimental Prototype}

The overall system interconnections are illustrated in Figure 4.9.
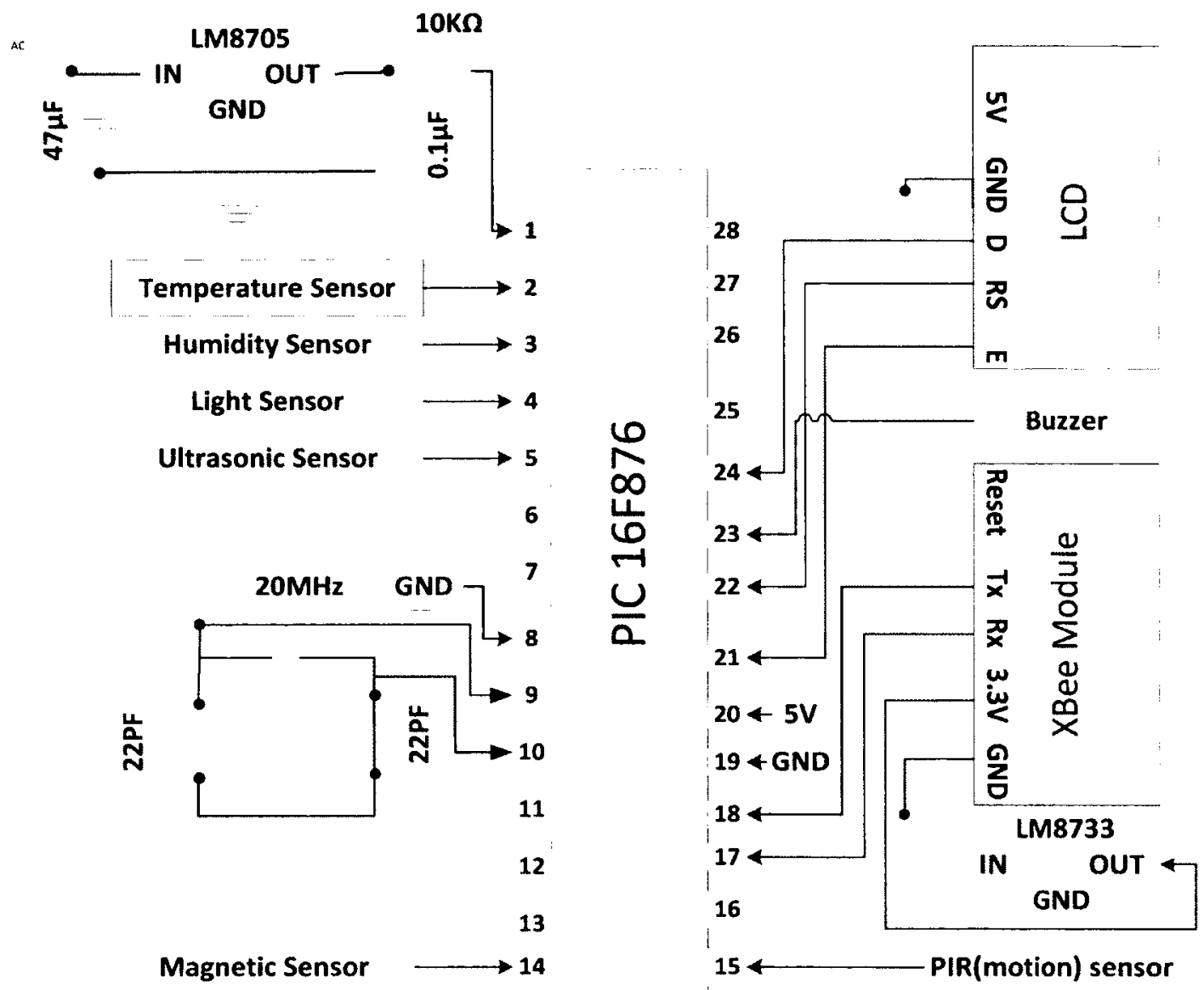

Figure 4.9: Pin details of PIC 16 F876 with system components 
Figure 4.10 is the actual prototype for the remote station we designed for the study. The remote station is equipped with its own $5 \mathrm{~V}$ regulated power supply, which can supply up to $1 \mathrm{~A}$ of load. The power supply to the remote system is given through $9 \mathrm{~V}$ regulated $\mathrm{DC}$ wall-power adapter or $9 \mathrm{~V}$ battery clip.

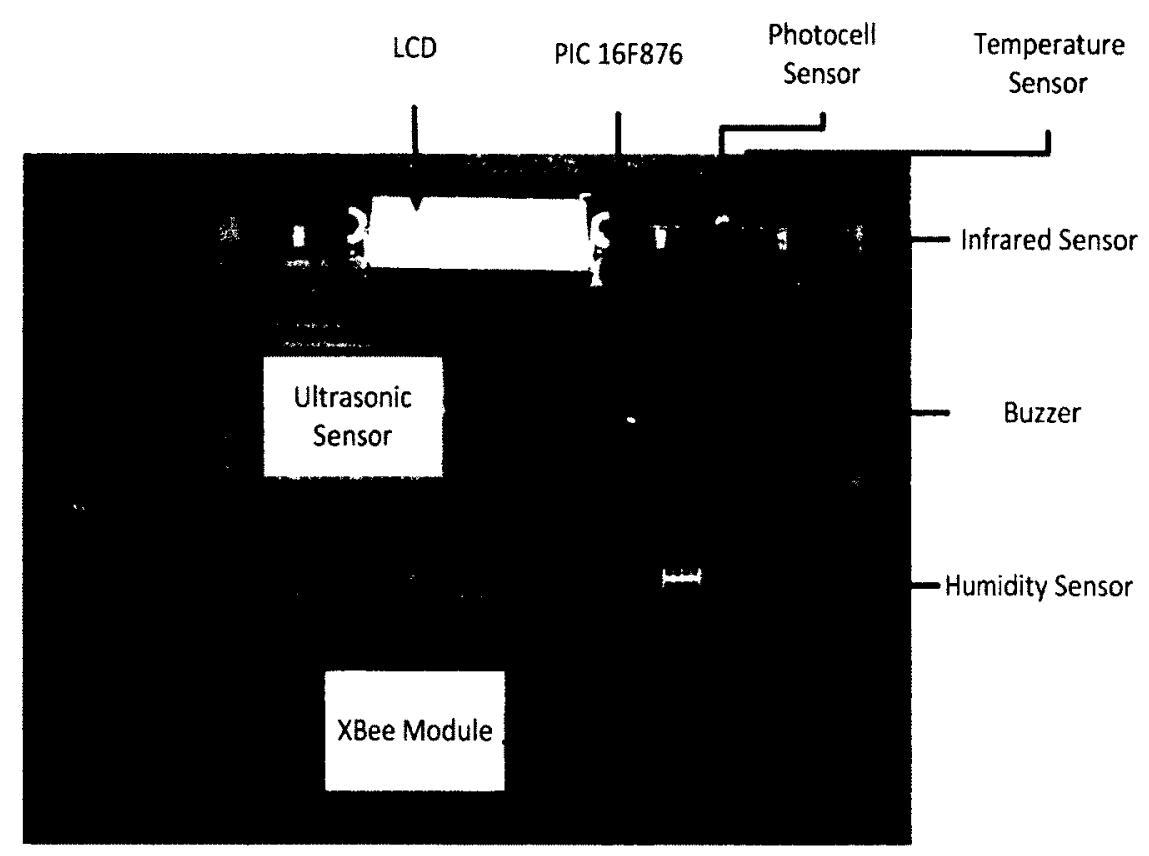

Figure 4.10: Remote station including sensors, LCD, microcontroller, buzzer, and XBee module

\subsubsection{The main experimental prototype components}

The main experimental prototype components include:

- On board 5V regulated supply;

- Microcontroller PIC 16F876;

- A $16 \times 2$ backlight liquid crystal display (LCD);

- XBee modules; 
- TMP36 temperature sensor;

- CHS - series humidity sensor;

- PDV - P8001 photocell sensor (light sensor);

- US5881LUA hall effect sensor;

- \#555 -28027 Infrared (IR) sensor;

- Buzzer;

- $20 \mathrm{MHz}$ oscillator;

- Capacitors;

- Resistors; and

- LED indicators.

\subsection{Software}

\subsubsection{Introduction}

The purpose of this subsection is to provide information on Proton IDE, PICKit2, X-CTU, and MATLAB, the software utilized for this thesis. The software were utilized for writing code, compiling and programming the microcontrollers, displaying collected data on the PC screen, and analyzing the data. The section is divided into four subsections. Section 4.6.2 provides step-by-step information on writing and compiling code. Section 4.6.3 provides information on the PICKit2 software used to load programming code into the microcontroller. Section 4.6.4 provides information on the programmer board used to load proton IDE onto the microcontroller. Section 4.6.5 provides information on the X-CTU window. This window is used to display our collected data on the PC screen. Finally, section 4.6.6 provides information on MATLAB. MATLAB was used to analyze our collected data. 
Table 4.4 below illustrates a brief description of these software packages.

\begin{tabular}{|c|c|c|c|}
\hline \multirow{2}{*}{ Software } & \multicolumn{3}{|c|}{ Feature } \\
\cline { 2 - 4 } & Version & Objective & Manufacturer \\
\hline X-CTU & -- & $\begin{array}{c}\text { Program XBee module and } \\
\text { collect data. }\end{array}$ & Digi International \\
\hline Proton IDE & Version 3.1 & Compile the program code. & Labcenter Electronics \\
\hline PICKit2 & $\begin{array}{c}\text { Version } \\
2.61\end{array}$ & $\begin{array}{c}\text { Download proton software } \\
\text { into microcontroller PIC } \\
\text { 16F876. }\end{array}$ & Microchip \\
\hline Matlab & $2007 a$ & Analyse collected data. & The MathWorks \\
\hline
\end{tabular}

Table 4.4: List of software used for the research

\subsubsection{Proton IDE: Compiler}

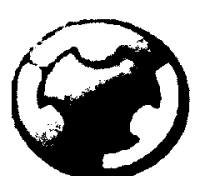

There are several methods of writing programming code onto a PIC microcontroller. These methods can be done through programming languages such as BASIC, C, or Assembly Language. In our study, we chose Proton Basic Integration Development Environment (IDE) because of its features and integration option. The Proton (IDE) is a very popular programming language for microcontrollers. Proton IDE is developed and distributed by Crownhill Associates LTD. The programming language was created as a result of collaboration among three British companies, Crownhill Associates, Mecanique, and Labcenter Electronics. The common goal was to develop a best breed of product that could:

- Provide an affordable, comfortable, and powerful development environment for the most popular microcontrollers: the Microchip PIC ${ }^{\otimes C U}$;

- Suit all levels of users from outright beginner to professionals;

- Allow for the development of programming code in a state-of-the-art development environment; and 
- Compile the program code and view the resulting assembly language commented with the program code.

After installing the Proton (IDE) software, the window shown in Figures 4.11 and 4.12 was used to write and compile our programming code and save it onto the PC hard drive so that it could be loaded into the microcontroller via software called PICKit2.

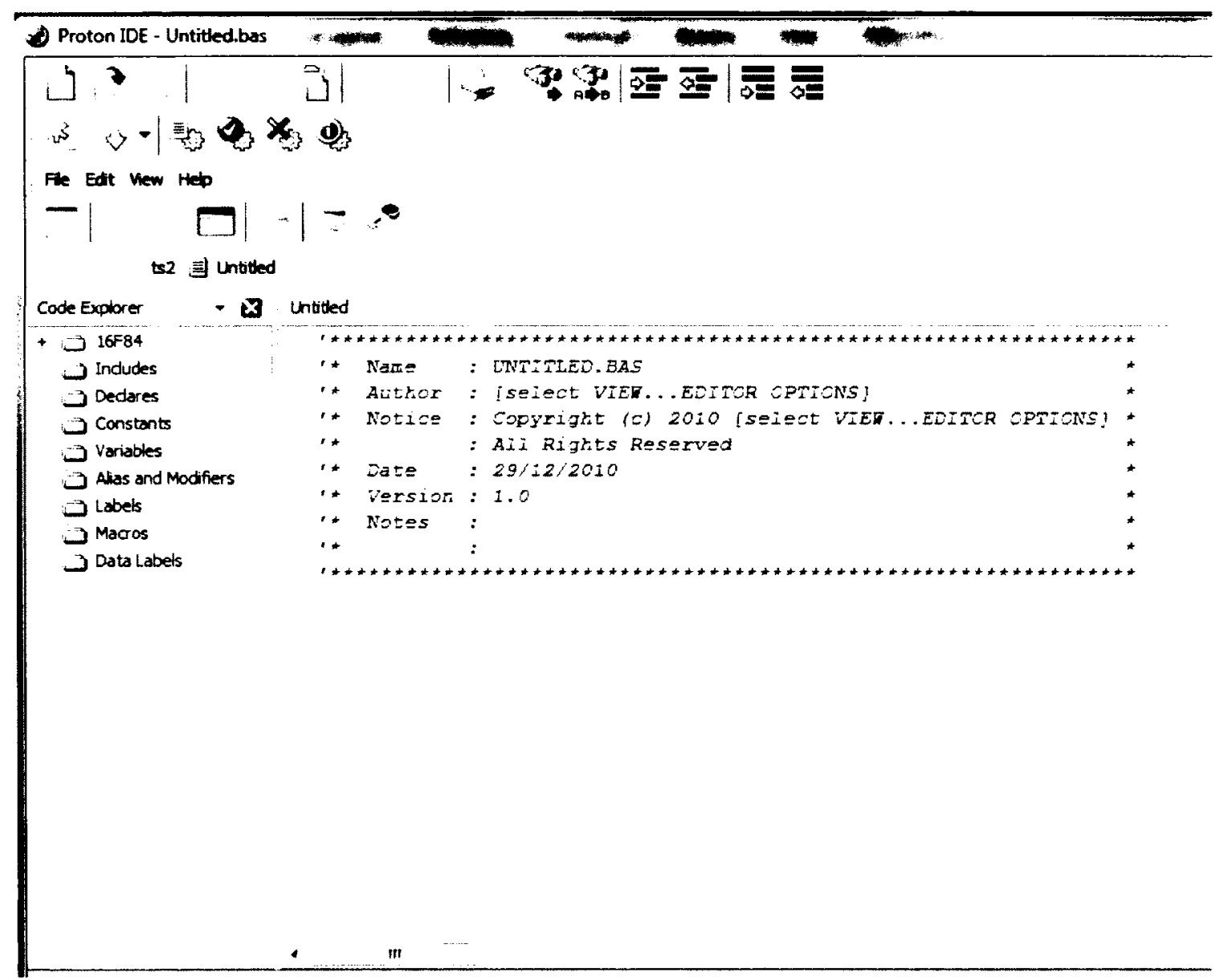

Figure 4.11: Proton IDE 


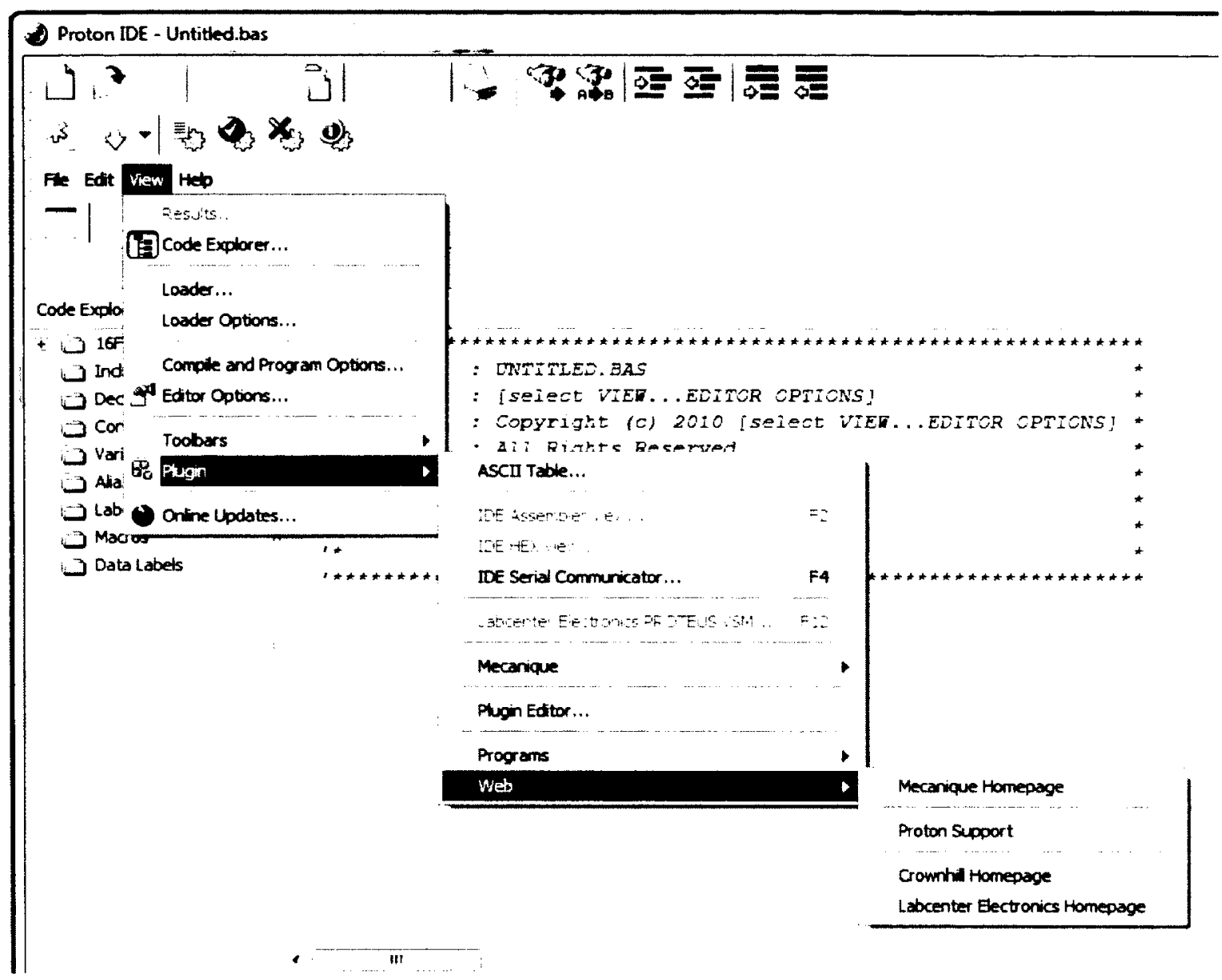

Figure 4.12: Proton IDE with features

\subsubsection{PICKit2: Programming Microcontroller}

After all of the logical errors were resolved, the programmable tool PICKit2 (shown in Figure 4.13) was used to download the proton IDE programming code into the microcontroller. The hex file was loaded into the microcontroller via the microcontroller programmer hardware as shown in Figure 4.13, Figure 4.14, and Figure 4.15. 


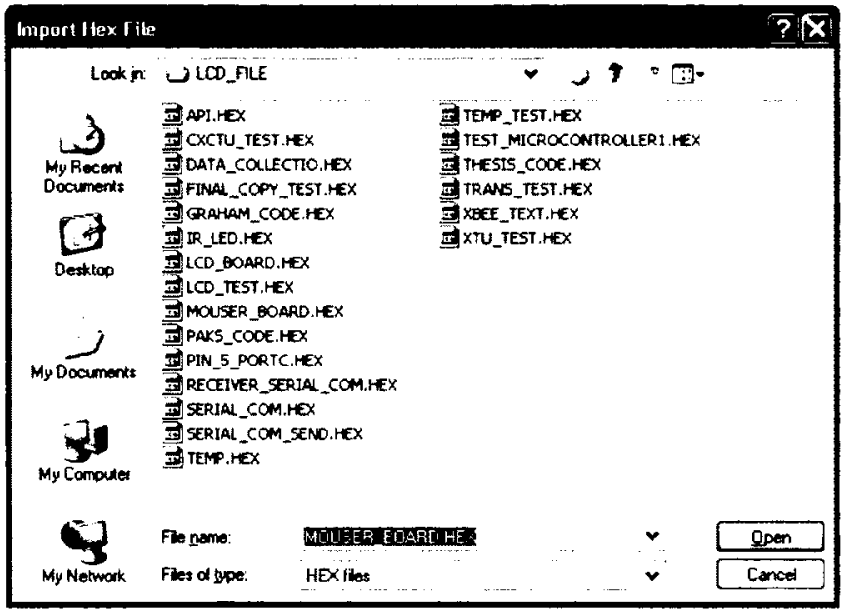

Figure 4.13: HEX file to be loaded into PICKit2

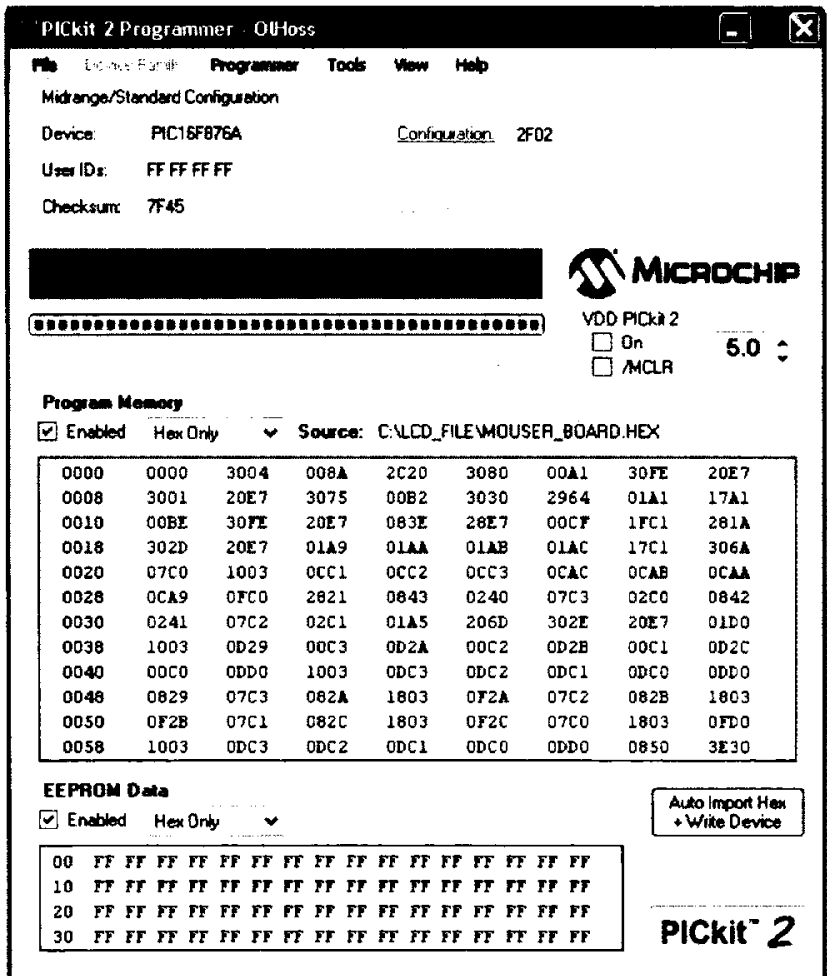

Figure 4.14: PICKit2 is used to load the hex file into the microcontroller via microcontroller programmer hardware 


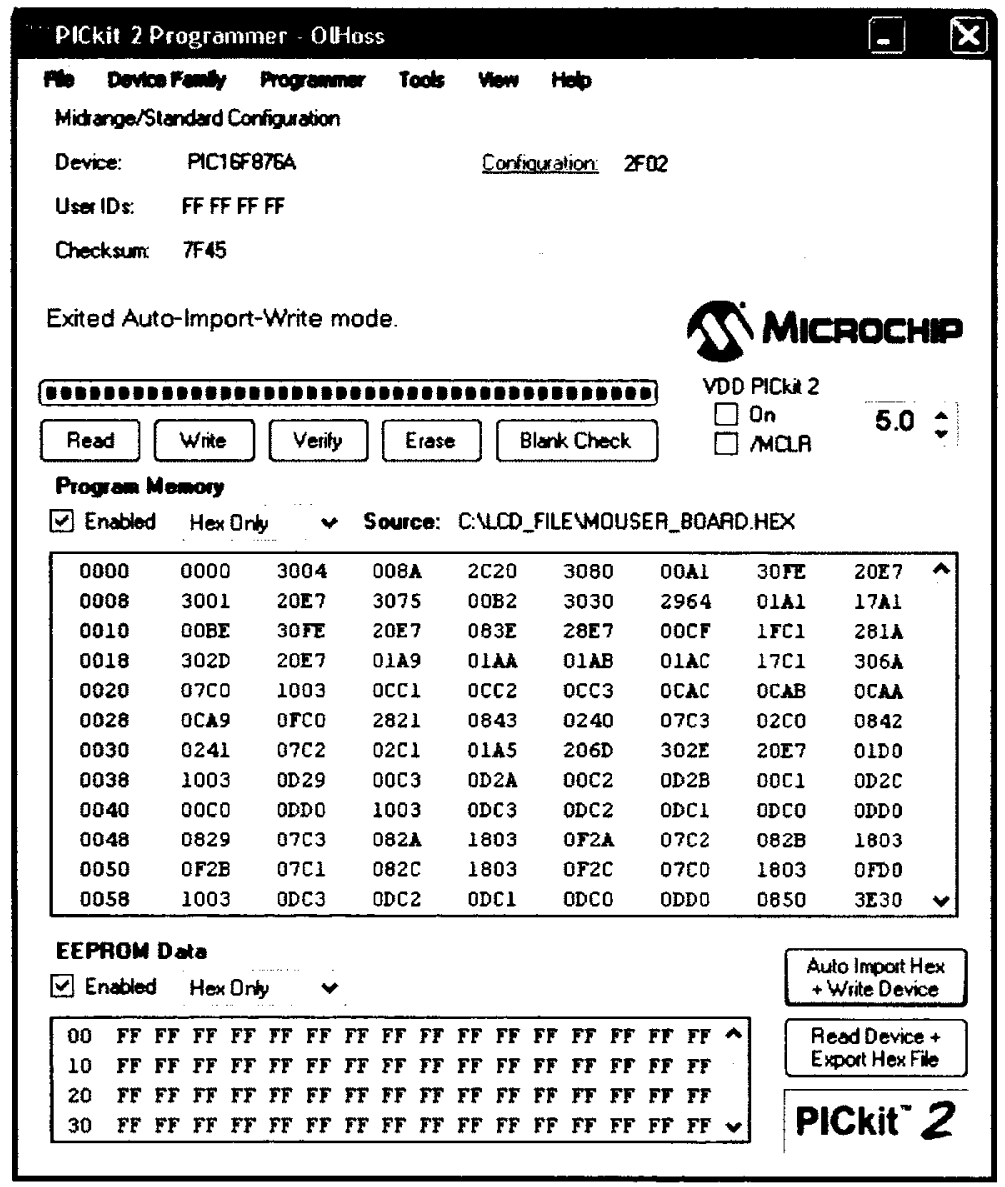

Figure 4.15: PICKit2 is used to load the hex file into the microcontroller via microcontroller programmer

hardware

\subsubsection{UK1300 USB PIC Programmer}

The UK1300 USB PIC Programmer shown in Figure 4.14 is the type of a programmer we used to download proton IDE program into the microcontroller. The programmer board includes $\mathrm{ZIF}^{15}$ (zero-force-insertion)

\footnotetext{
${ }^{15} \mathrm{ZIF}$ is an acronym for zero insertion force, a concept used in the design of IC sockets, invented to avoid problems caused by applying force upon insertion and extraction.
} 
adapter sockets. The socket board allowed us to remove and insert the PIC 16F876 easily and quickly. The programmer was selected for the thesis because it:

- Supports practically all current pic microcontroller, 8 to 40 PIC pins;

- Does not require a power supply. Power is supplied directly from the USB Port;

- Is $100 \%$ compatible with PICKit 2 interfaces; and

- Saves a considerable amount of time and protects microcontroller pins from damage.

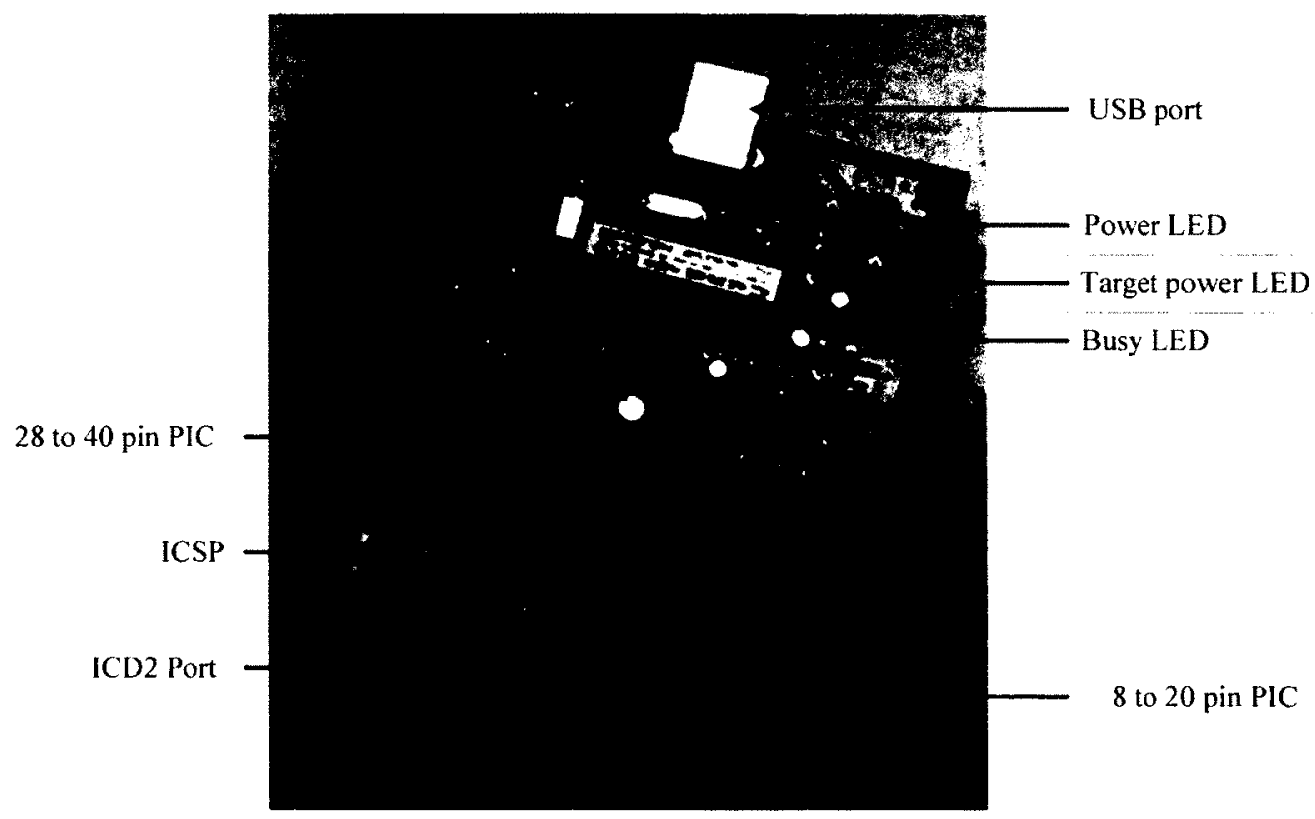

Figure 4.16: UK1300 - USB PIC Programmer for programming microcontroller

\begin{tabular}{|c|c|}
\hline Programmer & UK1300-USB PIC Programmer \\
\hline Support & 8 to 40 PIC pins \\
\hline Manufacturer & Cana-Kit Corporation \\
\hline
\end{tabular}

Table 4.5: UK1300 - USB PIC Programmer 
The device was connected to the computer to program our microcontroller, as shown in Figure 4.15.

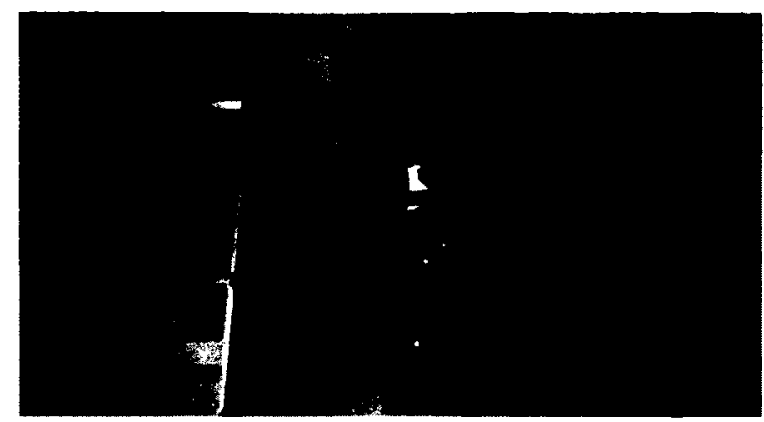

Figure 4.17: USB PIC Programmer connected to computer to program microcontroller

\subsubsection{X-CTU}

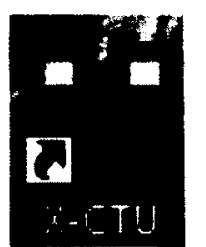

X-CTU is a Windows-based application for XBee software for configuration and test utility. The software is provided by Digi and designed to interact with the firmware files found on Digi's RF products and to provide a simple-to-use graphical user interface [62]. We used the software to configure the XBee radio and also to collect the data to be analysed. Figure 4.16 depicts X-CTU window. The "Terminal" tab is where collected data is displayed. "Select Com Port" is where the information on the type of XBee module connected to the Com Port is displayed. There is no XBee module connected in the Figure 4.16 to be displayed into the Com port. The "Modern Configuration" button is where the XBee module is configured. Firmware needs to be loaded to all ZigBee modules to form the network. X-CTU software is used configured ZigBee modules. X-CTU software program and installation instruction are available at http://ftp1.digi.com/support/documentation/90001003_A.pdf. In addition to X-CTU software, the appropriate driver for the XBee adapter board is also available at http://www.digi.com/support/productdetail?pid=3352. 
Here are the basic steps that must be followed to load firmware properly into the ZigBee module.

- Download and install the X-CTU software, and make sure the right version of the X-CTU software is installed.

- Connect the XBee board to computer Com Port via the USB adapter. This procedure will prompt the connected Com Port to appear on the X-CTU screen.

- Click the "New version" button to download the new version of the X-CTU software.

- After the new version is installed, click "Configure tab." This will load settings for the install module. In "Function Set" select the type of modern from the list.

- Once the firmware update is completed, click on the "Read" button to change a few parameters.

- To set ZigBee device as "Coordinator," the following parameters need to be changed:

- PAN ID = 234 (actual any PAIN ID number between 0x0 and 0xFFFFFFFFFFFFFFFF)

- Destination Address Low $=$ FFFF

- $\quad$ Node Identifier $=$ COORDINATOR

- Packetization Timeout $=25$

- The coordinator was programmed as a coordinator to synchronize the communication networks so that all of the wireless messages received by the antenna were echoed through the USB port to the computer.

- To set ZigBee device as "router," the following parameters need to be changed:

- PAN ID $=234$ (PAIN ID selected should be the same as in Coordinator)

- Destination Address Low $=$ FFFF

- $\quad$ Node Identifier $=\mathbf{R} 1 \ldots$..Rn 
- $\quad$ Packetization Timeout $=25$

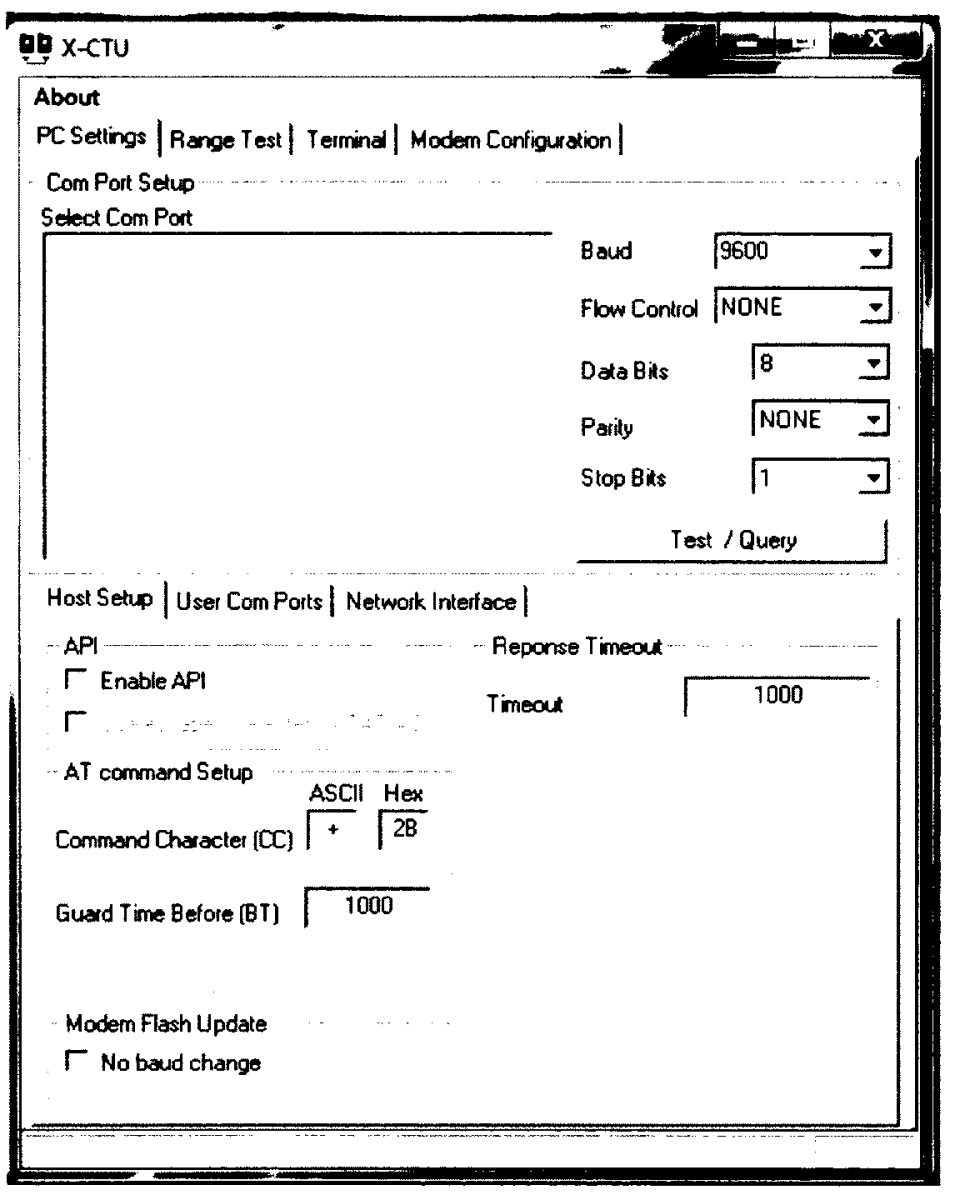

Figure 4.18: X-CTU Windows graphical user interface (GUI) for configuration and testing

$\mathrm{X}$-CTU is designed to operate strictly for the Windows platform. Windows $98,2000, M E, X P$, and Windows 7 are versions of Windows that are compatible with X-CTU. Window 95, NT, UNIX, or Linux are not compatible with XCTU. However, there is other software that can be used for the following operating systems, such as Coolterm, HyperTerminal, TeraTerm, Zterm, or Screen. 
- CoolTerm: Is an open source serial terminal program created by Roger Meier ${ }^{16}$ that runs well on both Windows and Macintosh.

- HyperTerminal: Is a serial terminal program that can be found in Windows XP and other older versions of Windows operating systems.

- Tera Term: Is a free open source window that has many functions, including a serial communication terminal.

- Zterm: Is a Macintosh serial terminal program.

- Screen: Is suitable for UNIX and Macintosh serial terminal programs.

${ }^{16}$ Roger Meier is a person who began writing his own program as a hobby when he could not find any decent firewall or shareware for certain tasks he wanted to use on a computer. 


\section{CHAPTER 5}

\section{TESTING AND RESULT}

\subsection{Introduction}

This chapter is organized into three sections: Section 5.1 provides an introduction to the chapter. Section $\mathbf{5 . 2}$ presents information on the testing of the prototype system at different locations. Finally, section 5.3 focuses on the results of collected data in the prototype apartment.

\subsection{Testing the Prototype system at Different Locations}

This section explains the testing process of the indoor and outdoor range of the prototype system, which was done to determine the accuracy and functionality of the system indifferent environments. Appendix $\mathrm{E}$ provides information related to the testing of each sensor for their functionality prior to their usage for the research.

The section is divided into five subsections: section 5.2.1 provides a brief description of the Portable Radio Communication Lab. Section 5.2.2 discusses the indoor testing of the system. Section 5.2.3 reflects on the data collected at the Portable Radio Communication Lab (4050MC). Section 5.2.4 focuses on the outdoor testing of the system for accuracy and functionality in different environment. Finally, section 5.2 .5 focuses on the comparison of the outside collected weather data of the prototype system to an Ottawa weather report from Google and Yahoo websites 


\subsubsection{Laboratory Portable Radio Communication}

The experiment was conducted at the Portable Radio Communication Lab (4050MC) in the Minto Building. The Lab is located on the $4^{\text {th }}$ floor of the Minto Building, $4050 \mathrm{MC}$. The lab is about 40 feet by 40 feet and equipped with all of the necessary tools for the research.

\subsubsection{Indoor Test Case}

Test purpose: To test the range of the prototype system. The field testing for the range of the prototype system was tested at different locations of the Minto Building (MC), both inside and outside of the building. Figure 5.1 illustrates indoor testing range of the system at different locations of the $4^{\text {th }}$ floor of the MC Building.

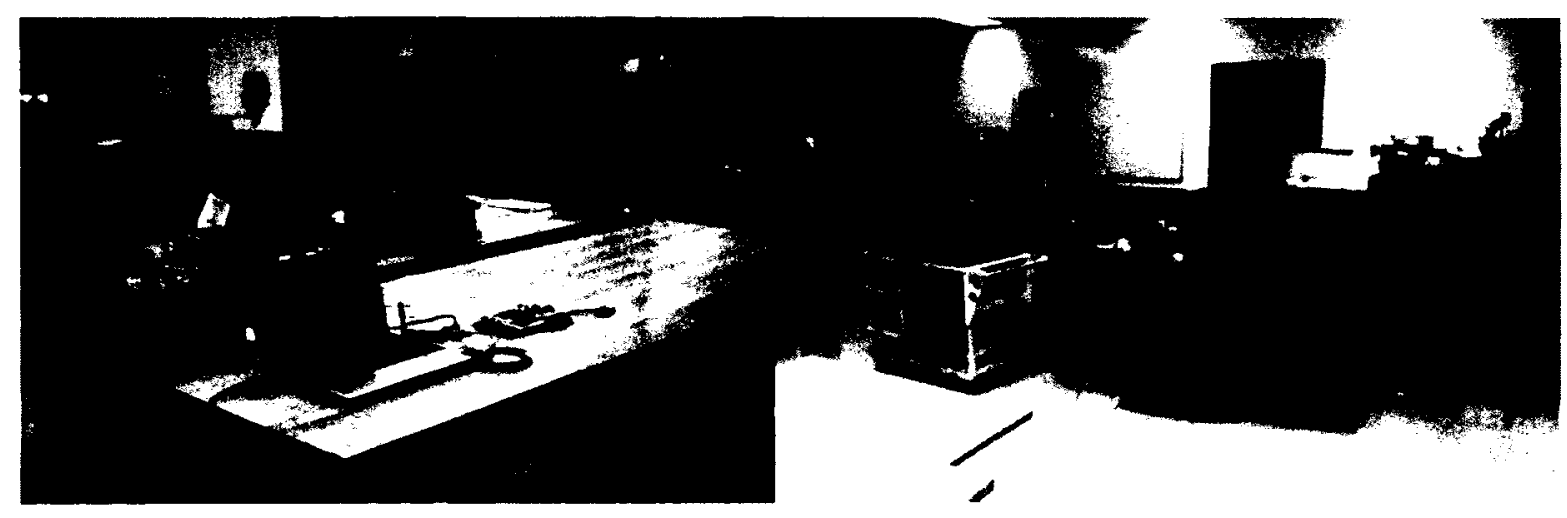




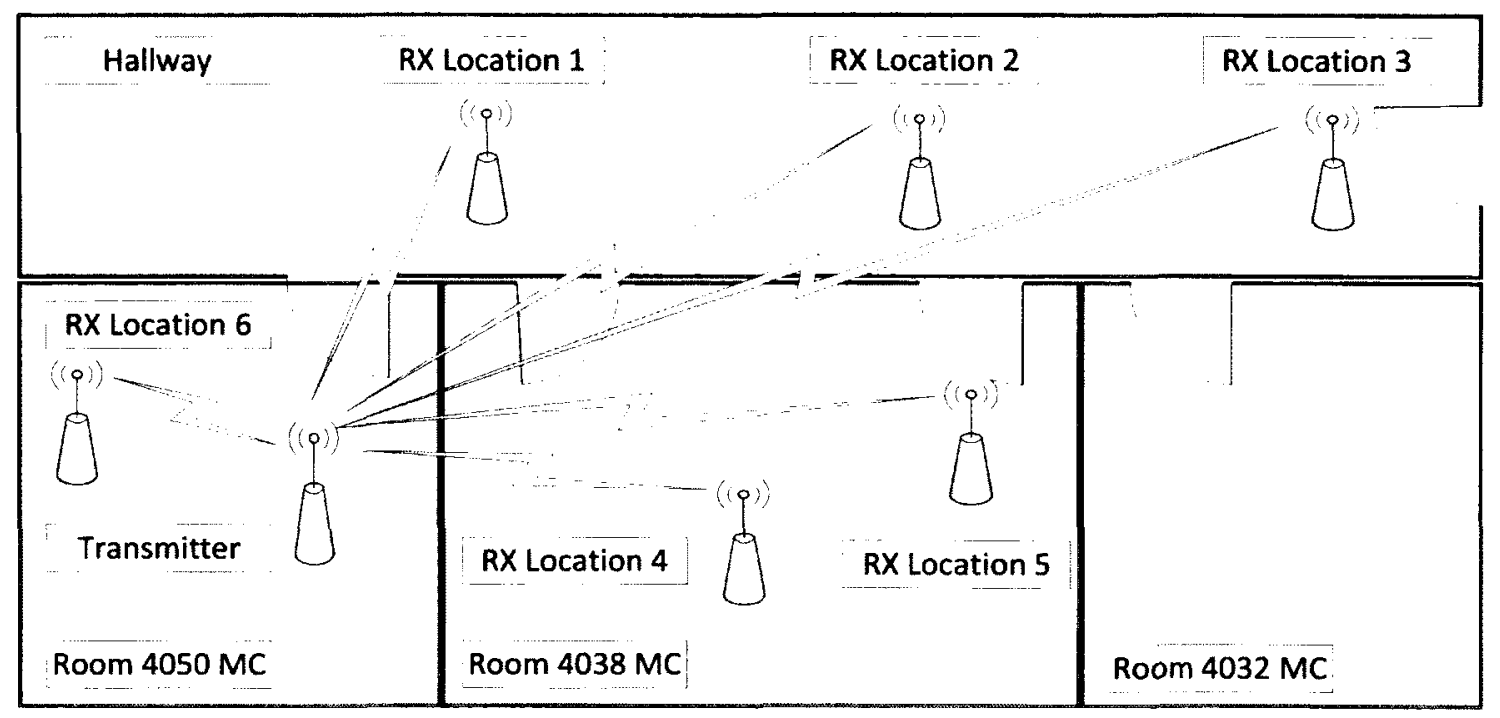

Figure 5.1: Prototype system testing locations at $4^{\text {th }}$ floor of the Minto Building

Expected result: The prototype system was expected to transmit data every two seconds within the XBee transmitter range. We also anticipated that fading, reflection, or interference would degrade the indoor performance of the prototype system. We also expected the throughput would decrease considerably when the distance between the transmitter and receiver reached a particular distance.

Test procedure: The transmitter (base) and receiver (remote) were set at distances of 10-100 feet from each other. The base station was positioned at different locations to test the range of the prototype systems, as shown in Figure 5.1. The station was then moved further and further away from the transmitter station until we reached at a distance that could no longer receive any data. The maximum distance at which XBee Series 1 can transmit data is 100 feet and the maximum distance at which XBee Series 2 can transmit data is 133 feet. As we were approaching this maximum distance, the throughput began to fail. 
Tested result: The results we obtained were as we had anticipated. As soon the XBee Series 2 was positioned at distance of the base station at location 3 and the XBee Series 1 was positioned at distance of the base station at location 5 (as illustrated in Figure 5.1), the prototype system performance degraded. Theoretical range as indicated by the product manufacturer data sheet for the XBee Series 1 is 100 feet and 133 feet for XBee Series 2; however, our experimental result showed that when the distance between transmitter and receiver range was 80 feet for XBee Series 1 and 100 feet for XBee Series 2 the performance of the prototype system dropped dramatically, as illustrated in Figure 5.2.

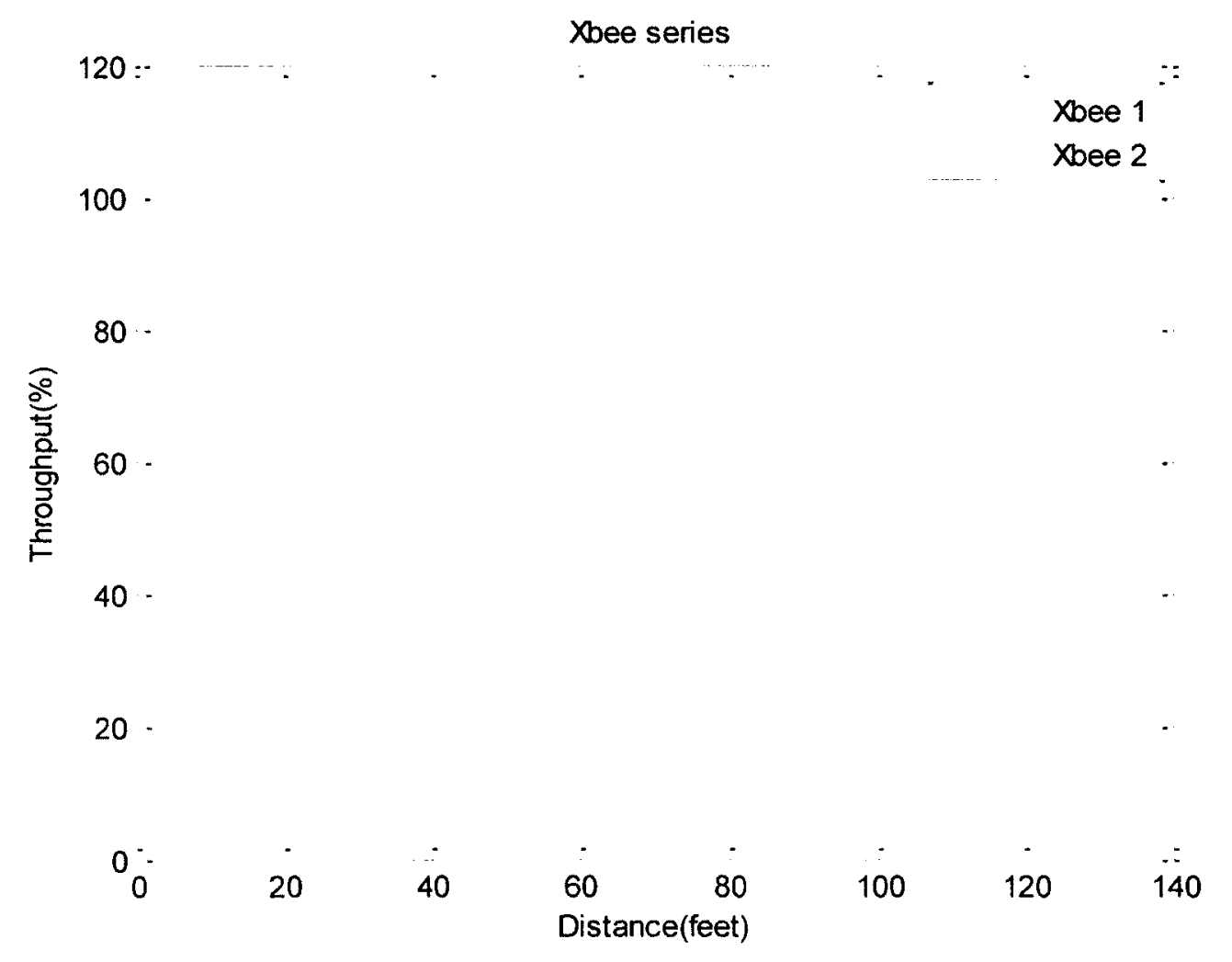

Figure 5.2: Range of XBee Series on 4th floor of the Minto Building 


\subsubsection{Collected Data at the Portable Radio Communication Lab}

The collected data at the Portable Radio Communication Lab $4050 \mathrm{MC}$ consists of the room temperature, uitrasound distance, light, and humidity. The collected data at the Portable Radio Communication Lab is shown in Figure 5.3.

- Light: 4.154 was indicated as the level of light in the room at the time (2:00 PM). The data was collected using the light sensor. A value of 5 is the maximum level of brightness to which the light can be turned on and 0 is the minimum value of darkness when the light is turned off.

- Temperature: 24.780 was the temperature of the lab in Celsius.

- Ultrasound: 63.837 was the distance from the workbench of the research to the ceiling of the lab in inches.

- Humidity: $29.325 \%$ was the humidity of the lab at time that the data was obtained as determined by the humidity sensor. 


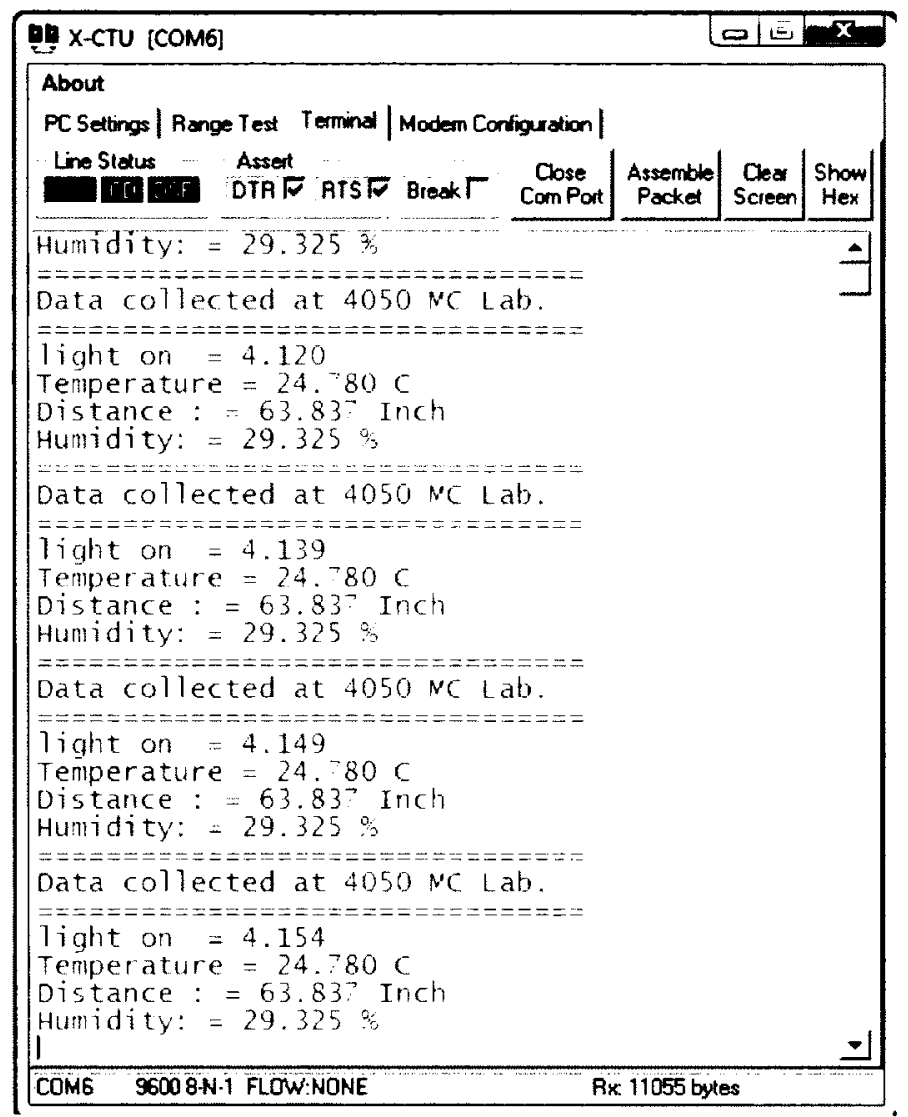

Figure 5.3: $4050 \mathrm{MC}$ laboratory collected data

\subsubsection{Outdoor Test Case}

Test purpose: The purpose of the outdoor test was to determine the accuracy and functionality of the system in different environments.

Expected result: The device was expected to transmit data every two seconds and operate at any condition.

Test procedure: The transmitter station was placed outside of the Minto Building at below zero temperatures for 20-30 minutes to transmit data within the system transmission range to the receiver station. The receiver station was placed inside the Minto Building at a distance of about 30 feet from the transmitter. 
Tested result: The result we received was as we had anticipated. This result is shown in Figure 5.4, which consists of outside temperature and humidity values at the particular time of day that the data was gathered

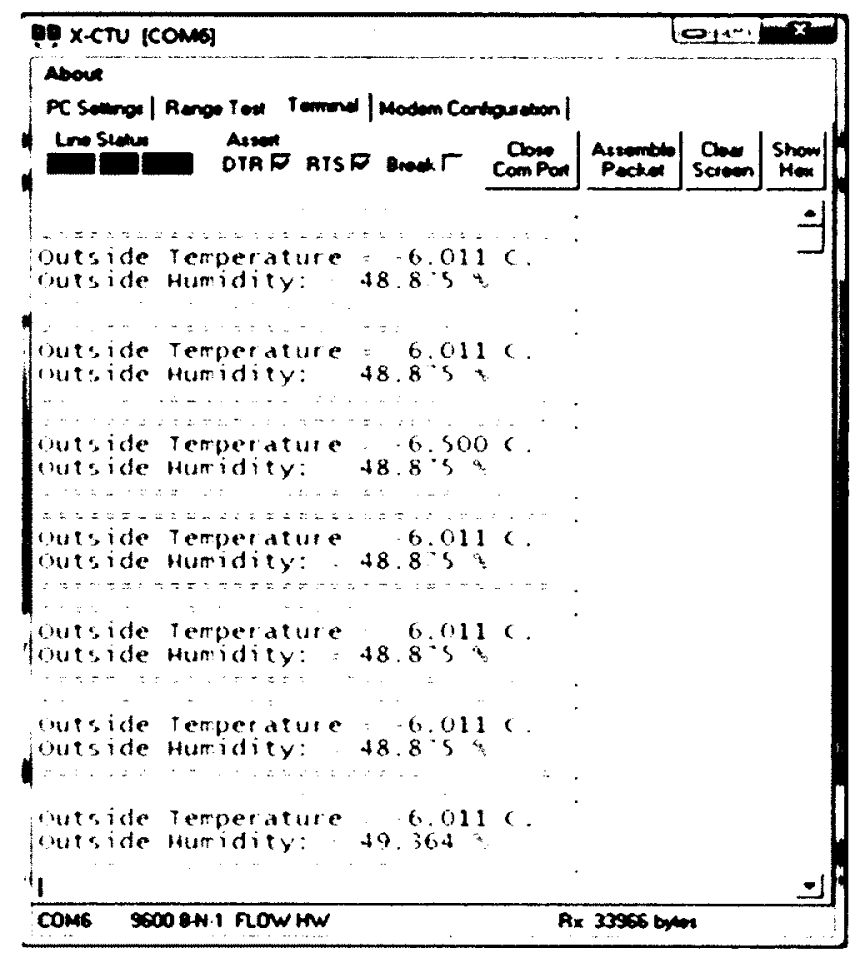

Figure 5.4: Outside temperature and humidity results

- Temperature sensor: -6.011 was the outside temperature in Celsius.

- Humidity sensor: $48.875 \%$ was the outside relative humidity.

\subsubsection{Ottawa Weather Report}

The collected data in Figure 5.4 were compared with the weather report of the city obtained from Google and Yahoo sites in Figure 5.5 to determine the accuracy and functionality of the prototype system. Based on the 
results, we conclude that our results in Figure 5.4 are very close to the results in Figure 5.5 . A comparison of the results is shown in Table 5.1.

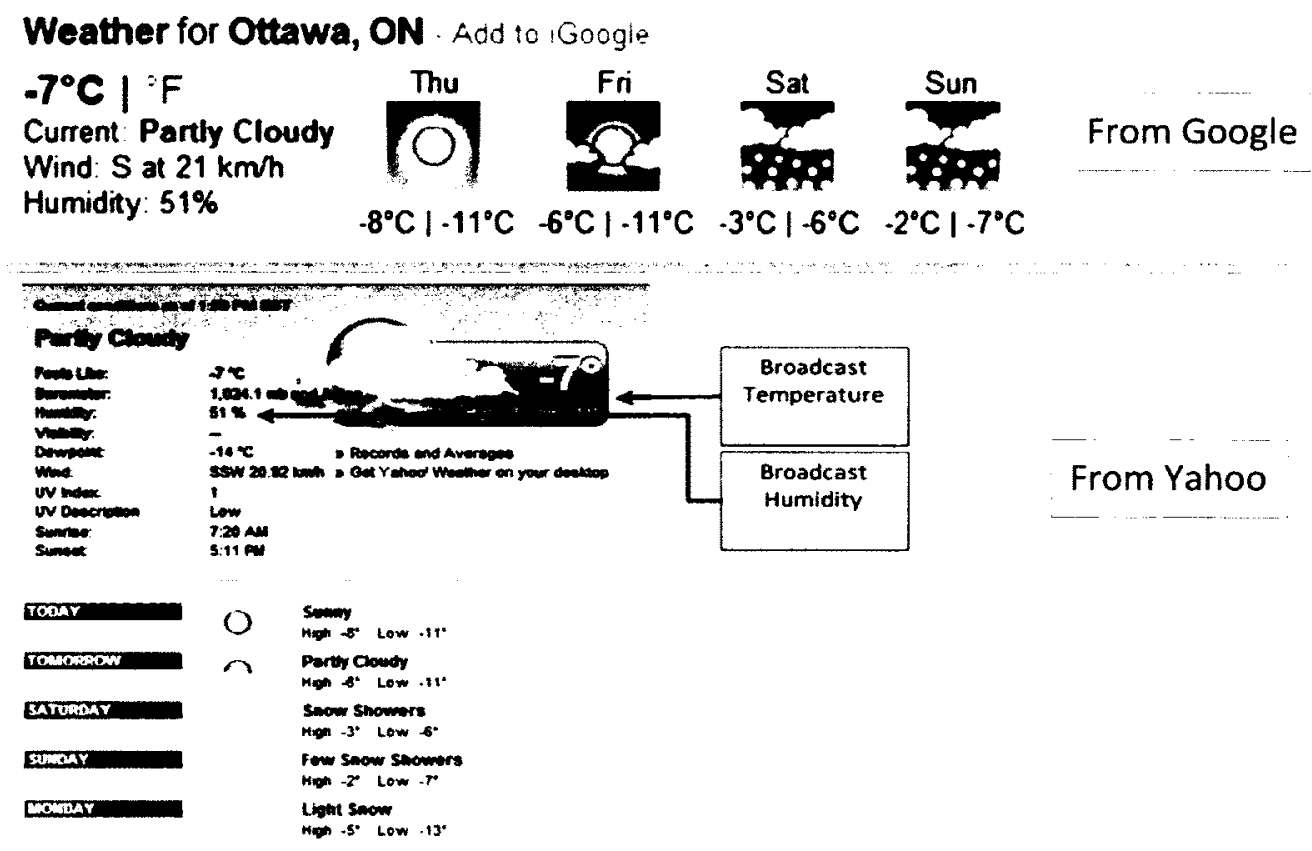

Figure 5.5: Ottawa weather report from Google and Yahoo websites

\begin{tabular}{|c|c|c|c|}
\hline Type & Research Result & Google & Yahoo \\
\hline Temperature $\left({ }^{\circ} \mathrm{C}\right)$ & -6.011 & -7 & -7 \\
\hline Humidity $(\%)$ & 48.875 & 51 & 51 \\
\hline
\end{tabular}

Table 5.1: Comparison of our research values with Google and yahoo websites weather report

\subsection{Prototype Apartment Results}

\subsubsection{Introduction}


The purpose of this section is to analyze our collected data from the different locations of the prototype apartment and various appliances in the apartment. The main goal of the section is to potentially identify an assortment of various Activities of daily living (ADL) such that a user can be alerted of any unacceptable change in behaviour of the occupant in the apartment.

The section is organized into seven subsections. Section 5.4.1 is the introduction; section 5.4.2 provides brief information on the data collection. Section 5.4 .3 provides brief discuss on the locations in the prototype apartment of the collected data. Section 5.4.3 discusses the data analysis. Section 5.4 .3 deliberates on the results of the data collected in the bathroom. Section 5.4 .4 discusses the results of the kitchen collected data. Section 5.4 .5 reflects on results of the refrigerator collected data. Section 5.4 .6 deliberates on the results of the living room collected data, and finally section 5.4 .7 describes the results for the balcony collected data. Section 5.4.8 discusses the Activities of daily living and the actual data interpretations of events of these the activities of daily living.

\subsubsection{Data Collection}

The data collection occurred through a laptop computer and sometimes through a desktop computer. The collection of the data can be divided into two parts: hardware and software.

- Hardware: The hardware consisted of a remote station and the base station, as shown in Figure 5.6.

- Remote station: The remote station consisted of three major components: six non-invasive sensors, LCDs to display data locally, and an XBee module to transmit data to the base station wirelessly. In our case, since line power was available where the remote station was placed in the apartment, the remote 
station was plugged into electrical outlets via a DC adapter (wall wart), as shown in Figure 5.7. Even though the station was not portable in this situation, data transmission still occurred in a wireless manner.

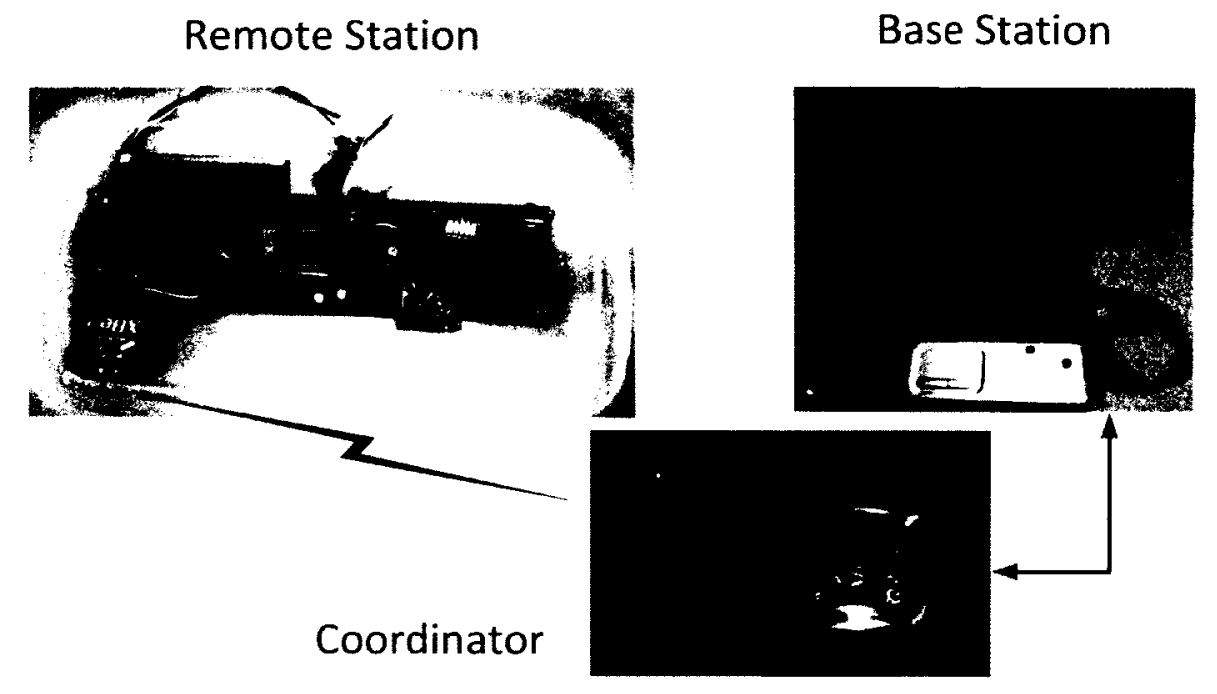

Figure 5.6: The system hardware consisted of a remote station and a base station

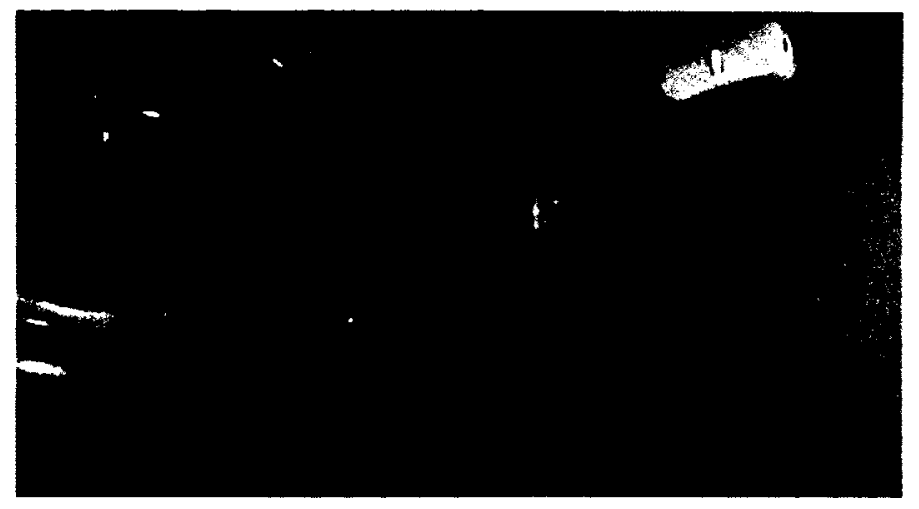

Figure 5.7: The remote station connected to the electrical outlet 
- Base station: The base station consisted of a coordinator connected to a desktop computer or laptop. The coordinator was connected to a computer through a USB connector. The coordinator collected data from the remote station and relayed it to the computer where the data were stored. The sensors data fusion were recorded according to changes in events (such as active or inactive), physical characteristics, moisture content of the environment, or speed or vector of an object or objects in the field of view.

- Sensors: The following six non-invasive sensors were used to collect data for analyses:

- Infrared Sensor

- To target mobility of humans in specific apartment areas, such as kitchen, washroom, living, and bedroom.

Light Sensor

- To detect the switching on/off of lights.

- To detect lightness and darkness.

- To determine use of appliances, such as the refrigerator.

- Temperature Sensor

- To measure ambient temperature.

- To detect use of the shower and toilet.

- To detect use of the electrical appliances.

- HumiditySensor

- To measure ambient humidity.

- To detect use of the shower and toilet.

- To detect use of the electrical appliances. 
- Magnetic Sensor

- To identify the opening and closing of doors.

- To identify use of major appliances, such as the refrigerator.

- Ultrasound

- To measure the distance between objects.

\subsubsection{Data Collection Locations}

This section focuses on the results of the data collected at the following locations of the prototype apartment: kitchen, living room, washroom, and balcony, as shown in Figure 5.8. The data for the apartment appliances, such as the fridge and stove, were also collected and analysed.

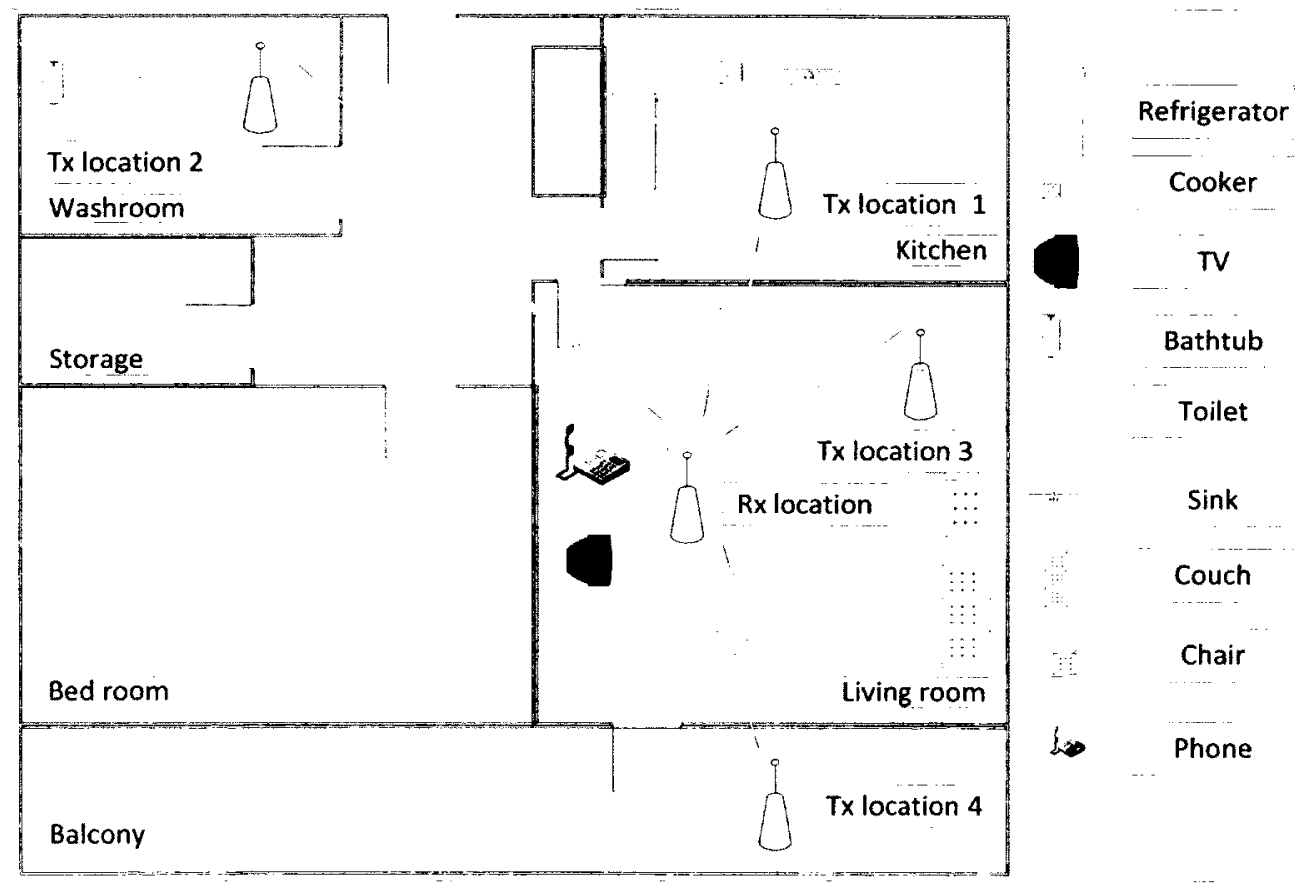

Figure 5.8: The prototype apartment 
Table 5.3 shows the types of sensors used to collect data at different locations in the prototype apartment.

\begin{tabular}{|c|c|c|}
\hline Locations & Sensor & Monitoring \\
\hline Bathroom & \multirow{3}{*}{$\begin{array}{l}\text { thermistor, humidity, } \\
\text { Photocell, PIR motion }\end{array}$} & temperature, humidity, light, bathroom motion \\
\hline Kitchen & & temperature, humidity, light, kitchen motion \\
\hline Living room & & temperature, humidity, light, living room motion \\
\hline Refrigerator & $\begin{array}{l}\text { thermistor, humidity, } \\
\text { Photocell, hall effect }\end{array}$ & temperature, humidity, light, refrigerator door \\
\hline Balcony & $\begin{array}{l}\text { thermistor, humidity, } \\
\text { Photocell }\end{array}$ & temperature, humidity, light \\
\hline
\end{tabular}

Table 5.2: Location and sensor used to collect data in the apartment

\subsubsection{Data Analysis}

This subsection discusses the detection of events from different sensors. Each event was given a symbol to identify it, as shown in Table 5.3. The goal of this subsection is to investigate events that occurred as a result of changes in the environment. The purpose of this subsection is to analyze our collected data of events of daily living of an occupant in the different locations of the prototype apartment that pertain to the usage of various appliances in the apartment. The analysis part is done using MATLAB. Figures $5.9,5.11,5.13,5.15$, and 5.17 illustrate the results of the data analysis. Table 5.3 illustrates the transition states of the sensors.

\begin{tabular}{|c|c|c|}
\hline Sensors & Transition state & Actions \\
\hline Motion & On - Off & A \\
\cline { 2 - 3 } & Off - On & B \\
\hline Light & On - Off & C \\
\cline { 2 - 3 } & Off - On & D \\
\hline Temperature & Rise - Fall & E \\
\hline
\end{tabular}




\begin{tabular}{|l|c|c|}
\hline & Fall - Rise & F \\
\hline Humidity & Rise - Fall & G \\
\cline { 2 - 3 } & Fall - Rise & H \\
\hline Magnetic & On - Off & I \\
\cline { 2 - 3 } & Off - On & J \\
\hline
\end{tabular}

Table 5.3: Transition states of sensors

\subsubsection{Bathroom Results}

Test purpose: The purpose of this section is to collect data about the changing events in the bathroom.

Expected result: The result was expected to indicate that temperature and humidity sensors detected an increase in temperature and humidity in the washroom when the bathtub was filling with hot water. Furthermore, we expected motion sensor to detect human motion in the washroom while the occupant was in the washroom filling the bathtub with the hot water.

Test procedure: The remote station was placed in the bathroom to transmit data every two seconds.

Tested result: The results we received were as we had expected.

Figure 5.9 illustrates the real-time monitoring of the physical activities of a user inside a bathroom.

- Before the user took a shower, Figure 5.9 indicates that:

* The temperature sensor detected that the temperature was at room temperature of approximately 23.8 degrees Celsius.

* The humidity sensor detected that the humidity reading was low; it was at approximately $28 \%$ relative humidity at 23.8 degrees Celsius. 
The light sensor spotted no indication that the light was turned on (event $\left({ }^{\prime} D\right.$ )) and no human motion (event $\left({ }^{\prime} B^{\prime}\right)$ ) or activities in the bathroom prior to the user taking a shower were identified by the motion sensor.

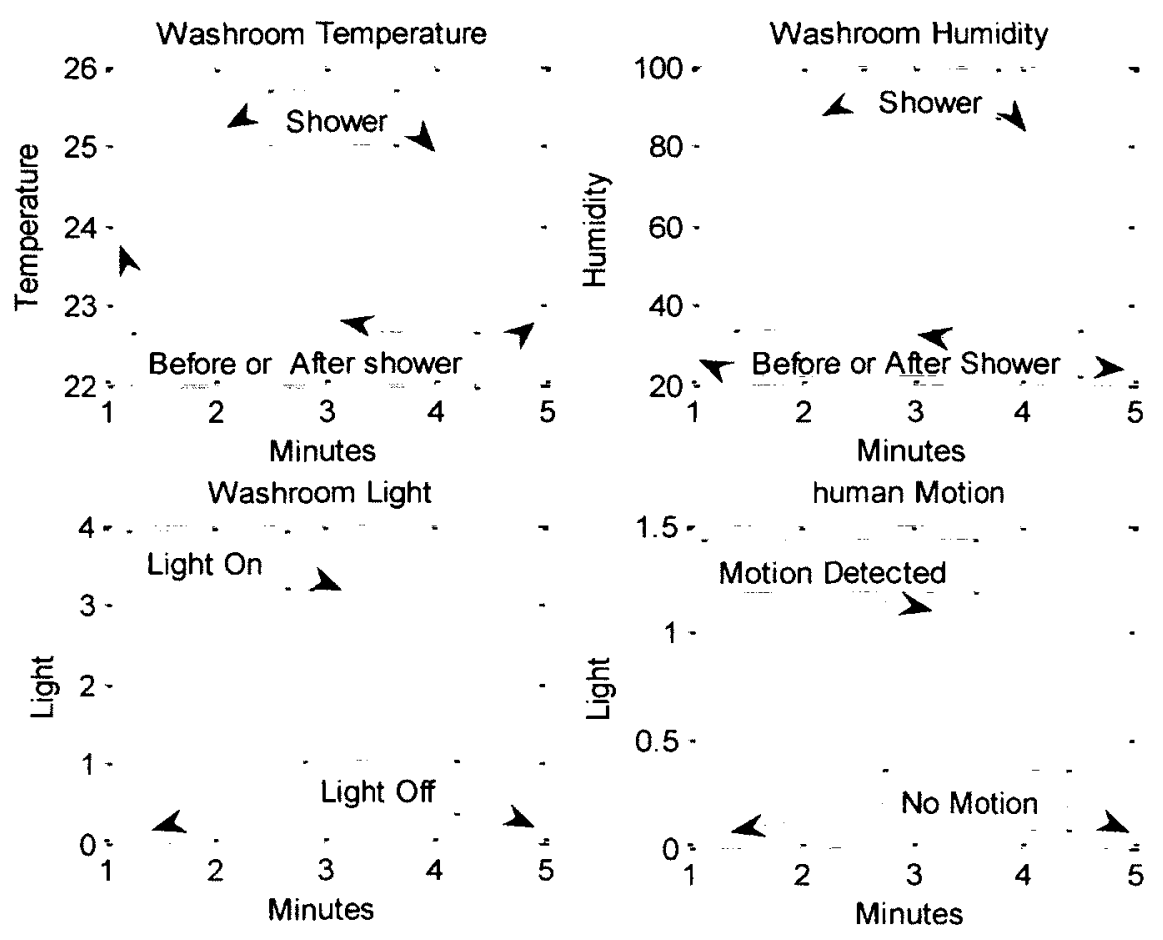

Figure 5.9: Graph of the data collected in the bathroom

- However, during the shower (as shown in the Figure 5.9), the prototype system temperature and humidity readings in the washroom indicated that:

The temperature sensor detected an increase in the washroom temperature to 25.4 degrees Celsius (event ('E')). 
* The humidity sensor noticed the humidity increased to approximately $88 \%$ of relative humidity at 25.4 degrees Celsius (event ('G')).

* The motion sensor also showed that there was an indication of human motion (event (' $B$ ')) in the bathroom during the shower period.

* The light sensor indicated that the bathroom light was turned on (event $\left({ }^{\prime}{ }^{\prime}\right)$ ) during the shower period.

- A few minutes after the user left the washroom, the temperature and humidity sensors showed the temperature and humidity dropped to its original level before the user had entered the washroom to take the shower.

* The temperature sensor detected the washroom temperature dropped to approximately 22.8 degrees Celsius (event ('F')).

- The humidity sensor noticed the washroom humidity also dropped to approximately $30 \%$ relative humidity at 22.8 degrees Celsius (event $\left.\left({ }^{\prime} H^{\prime}\right)\right)$.

* The motion sensor detected no human motion was present in the bathroom after the shower (event ('B')).

* The light sensor spotted no light present in the bathroom after the shower (event (' $D ')$ ).

We noticed that the temperature and humidity of the bathroom increased when the user was taking a shower. The figure also shows that the light in the bathroom was turned on and there was indication of the motion of a person in the washroom. These results indicate that during the collection of the data, there were some events going on in the washroom. 


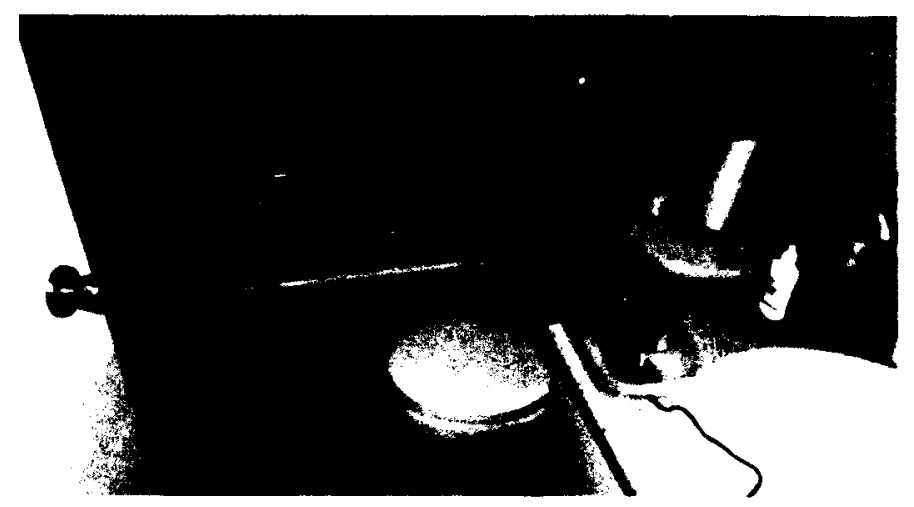

Figure 5.10: Remote station in bathroom collecting data

\subsubsection{Kitchen Result}

The kitchen is an area in house composed of various appliances, such as the refrigerator, dishwasher, oven, cook top, microwave, toaster, etc.

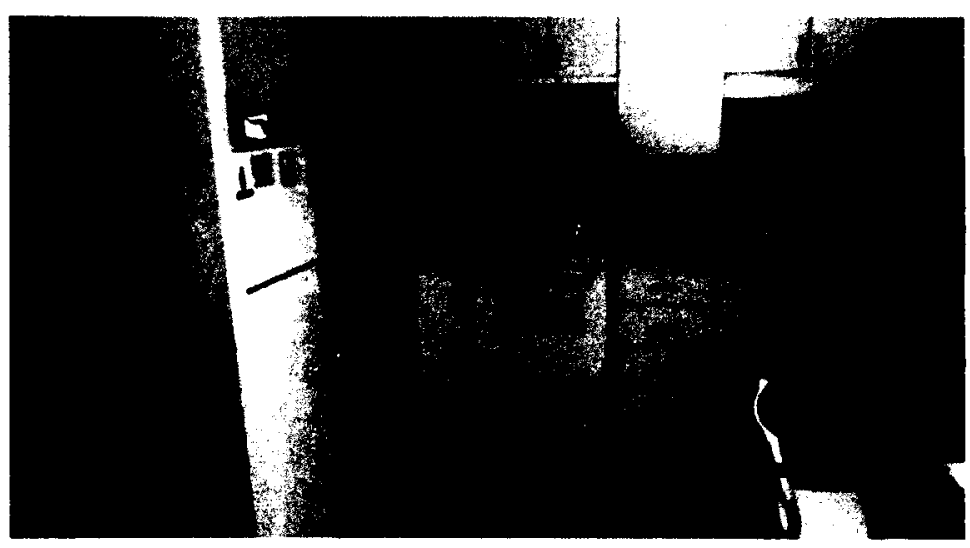

Figure 5.11: Prototype apartment's kitchen

Test purpose: The purpose of this section is to collect data of the events when kitchen appliances were used in the kitchen. 
Expected result: The results were expected to show that the temperature and humidity sensors detected an increase in temperature and humidity when oven was switched on to cook in the kitchen. Furthermore, we also expected that the motion sensor would detect human motion in the kitchen.

Test procedure: The remote station was placed in the kitchen to transmit data every two seconds.

Tested result: The results we received were as we had expected. Figure 5.11 illustrates the data of the events in a kitchen in real time monitoring when a kitchen appliance, such as the stove, was used. Figure 5.11 shows the results of the data in the kitchen before, during, and after the kitchen appliances were used.

- Before any detected of events in kitchen of appliances been used:

* The temperature sensor detected that the kitchen temperature was approximately 22.8 degrees Celsius.

* The humidity sensor identified that the kitchen humidity was at approximately $23.2 \%$ relative humidity at 22.8 degrees Celsius.

* The motion sensor showed no detection of human motion (event ('B')) inside the kitchen before the stove was turned on.

* The light sensor detected that there is no light (event $\left({ }^{\prime} C^{\prime}\right)$ ) present in the kitchen before any activities occurred. 


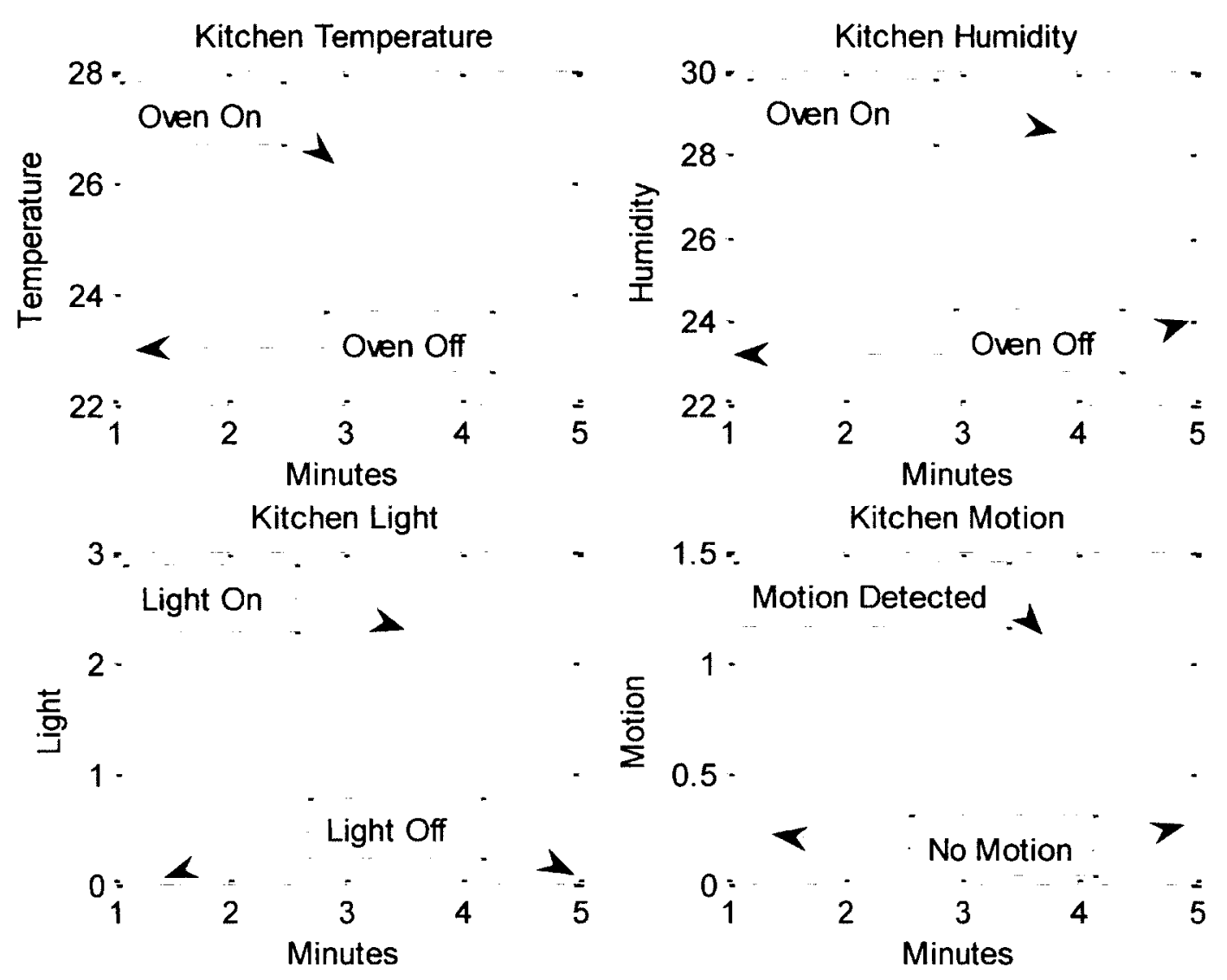

Figure 5.12: Graph of the data collected in the kitchen

- During the events in the kitchen - Figure 5.11 shows that when the stove was turned on to cook or boil water for tea or coffee, the temperature and humidity sensors detected that the kitchen temperature and humidity increased.

* The temperature sensor detected that the kitchen temperature increased to about 26.8 degrees Celsius (event ('E')). 
* The humidity sensor spotted that the kitchen humidity also increased to approximately $30 \%$ relative humidity at 26.8 degrees Celsius (event (' $\left.G^{\prime}\right)$ ).

* The motion sensor detected human motion (event ('A')) inside the kitchen when the stove was turned on.

* The temperature sensor detected that the temperature of the kitchen increased when the stove was switched on (event ('E')).

* The light sensor identified that there was also light present in the kitchen (event (' $C$ ')).

- After the event in the kitchen stopped:

* The temperature sensor detected that the kitchen temperature dropped to approximately 23.8 degrees Celsius (event ('F')).

* The humidity sensor noticed that the kitchen humidity also dropped to approximately $23 \%$ relative humidity at 23.8 degrees Celsius (event (' $\left.\left.\mathrm{H}^{\prime}\right)\right)$.

* The motion sensor detected that no human motion (event (' $B$ ')) was present in the kitchen after the stove was turned off.

* The temperature sensor detected that the temperature of the kitchen dropped after the stove was turned off (event ('F')).

* The light sensor indicated that the kitchen light was turned off (event ('D')). 


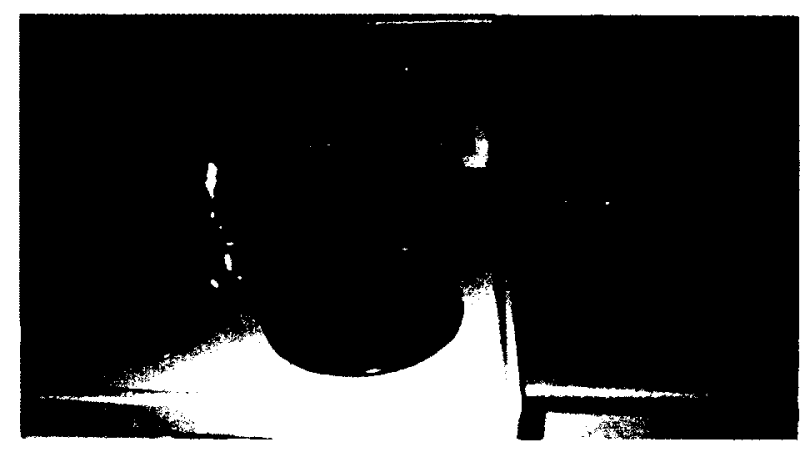

Figure 5.13: Remote station located in the kitchen collecting data

\subsubsection{Refrigerator Result}

Test purpose: The purpose of this subsection is to explain the procedure used to collect the refrigerator data in the kitchen.

Expected result: The results were expected to show that the temperature and humidity sensors would detect a decrease in temperature and humidity when the prototype system was placed inside the refrigerator for a few minutes. Furthermore, we expected that the motion would to show human motion present in the kitchen.

Test procedure: The remote station was placed inside the refrigerator to transmit data every two seconds. Tested result: The results we received were as we had expected.

Figure 5.13 illustrates the results of the collected data from the refrigerator in real time monitoring. 


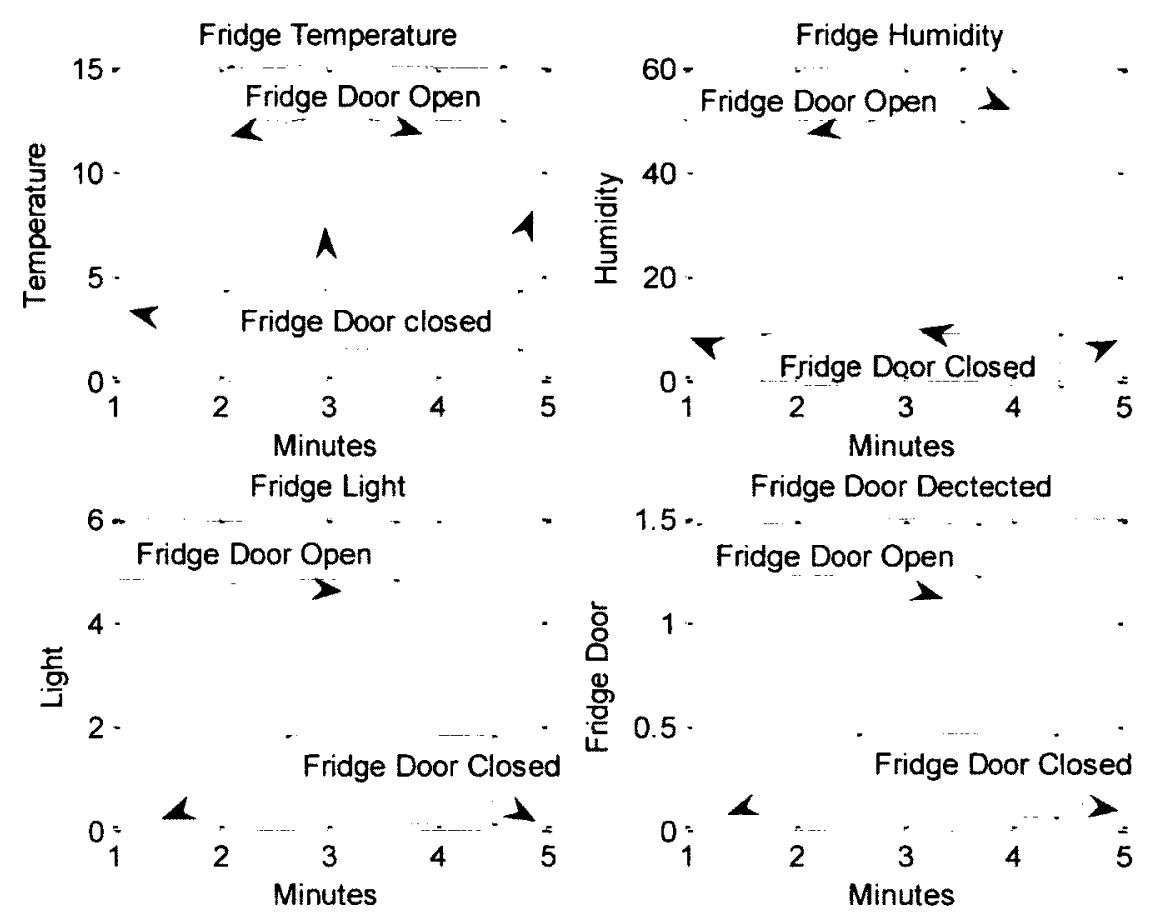

Figure 5.14: Graph of the data collected in the fridge

- When the remote station was positioned inside the fridge for few minutes and the fridge door was closed:

* The temperature sensor showed that the fridge temperature was set to 4 degrees Celsius.

- The humidity sensor showed that the fridge humidity was at $8 \%$ relative humidity at 4 degrees Celsius.

* The light sensor indicated that the fridge light automatically turned off (event ('D')) whenever the fridge door was closed.

- As soon as the fridge door was opened: 
* The figure indicates that the temperature sensor detected that the temperature of the fridge increased to 12 degrees Celsius (event ('E')).

* The humidity sensor also detected that the fridge humidity increased from $8 \%$ when the fridge door was closed to $42 \%$ relative humidity at 12 degrees Celsius (event (' $G$ ')).

* At the same time, the light sensor showed that the fridge light turned on automatically (event (' $\left.C^{\prime}\right)$ ) whenever the fridge door was opened.

* The magnetic switch sensor also showed that the fridge door was opened (event $\left({ }^{\prime}\left(I^{\prime}\right)\right.$ ).
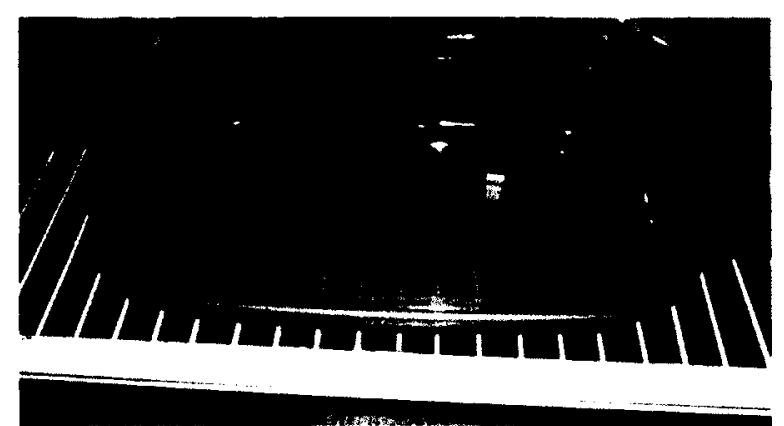

Figure 5.15: Remote station inside fridge collecting data

\subsubsection{Living Room Result}

Test purpose: The purpose of this section is to collect data of events in the living room.

Expected result: The result was expected to indicate events of daily living in the living room.

Test procedure: The remote station was placed in the living room to transmit data every two seconds.

Tested result: The results we received were as we had expected.

Figure 5.15 illustrates the collected data in real time in the living room of the apartment. 

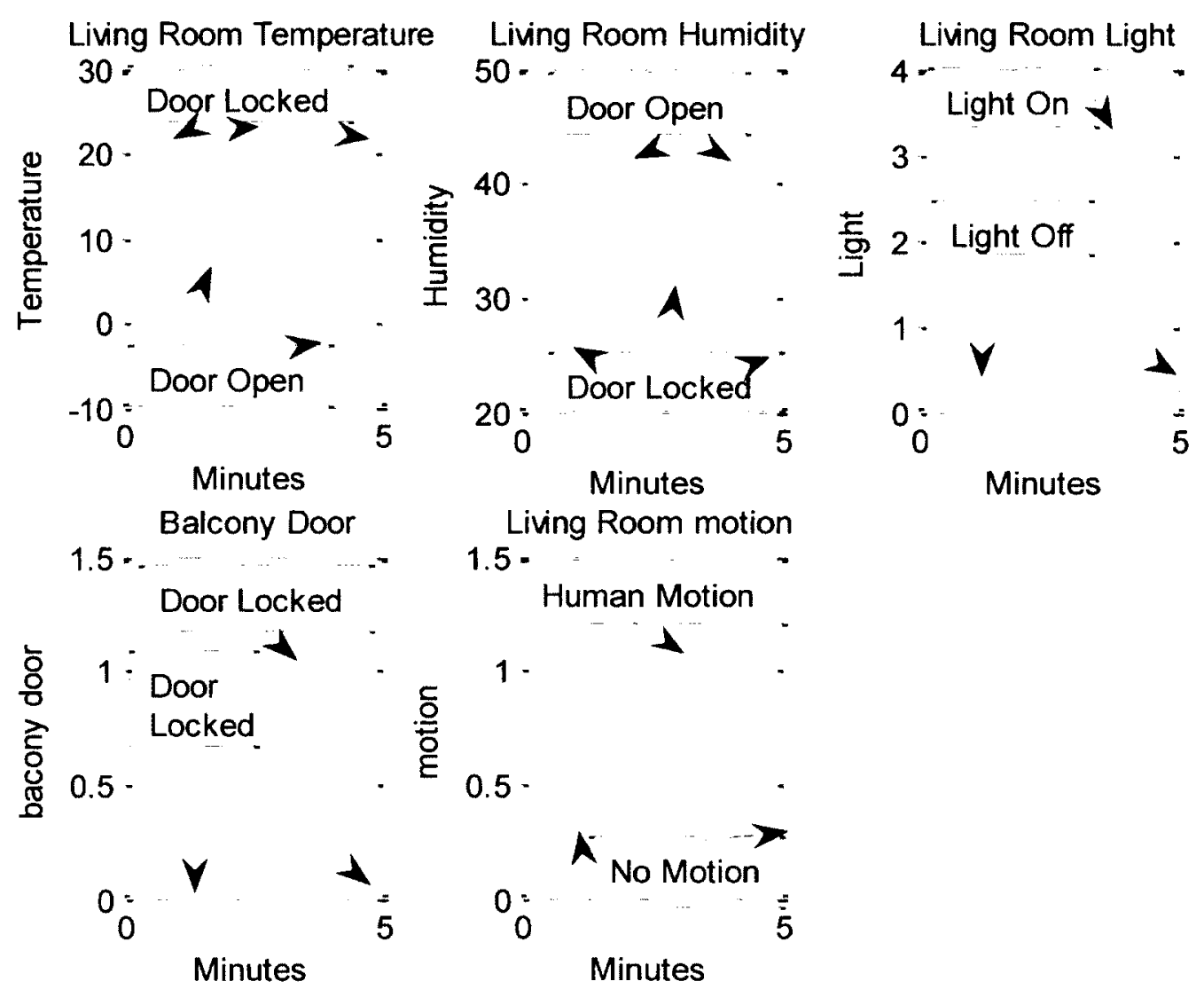

Figure 5.16: Graph of data collected from the living room of the apartment

As can be seen in the Figure 5.15:

* The temperature sensor detected that the living room temperature was at 22 degrees Celsius.

* The humidity sensor detected that the living room humidity was at $25 \%$ relative humidity at 22 degrees Celsius.

* There was also the detection of human motion in the living room (event (' $A$ ')) by the motion sensor. 
* There was also the sensor detection of light present inside the living room (event (' $\left.C^{\prime}\right)$ ).

As soon as the balcony door was opened as shown in Figure 5.16:

* The Figure 5.15 indicates showed the temperature sensor detected the temperature of the living room dropped to 4 degrees Celsius when the balcony door was opened for few minutes (event $\left.\left(F^{\prime}\right)\right)$.

* The humidity sensor detected the living room relative humidityalso increased to $42 \%$ relative humidity at 4 degrees Celsius (event ('G')).

* At the same time, the light sensor indicated that the living room light was turned on, (event $\left.\left({ }^{\prime} C^{\prime}\right)\right)$.

* The magnetic switch sensor also showed that the balcony door was opened (event $\left.\left({ }^{\prime} I\right)\right)$.

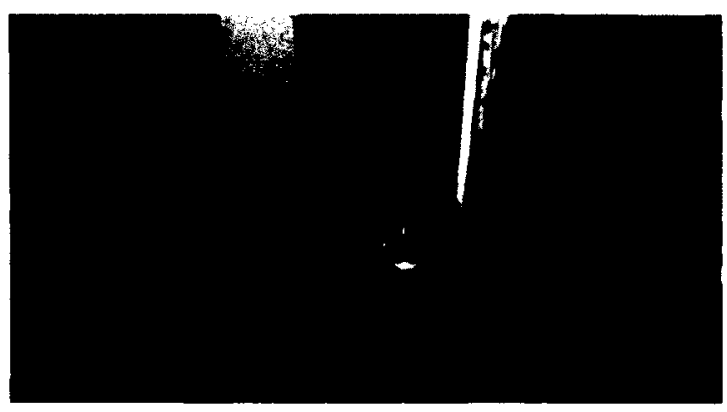

Figure 5.17: Living room when balcony door was opened

\subsubsection{Outside Results}

Test purpose: The purpose of the section is to collect data from the balcony of the apartment.

Expected result: The results were expected to indicate a decrease in temperature and an increase in humidity during the wintertime. 
Test procedure: The transmitter station was placed on the balcony of the apartment to transmit data every two seconds.

Tested result: The results we received were as we had expected, the prototype system indicated a drop in temperature outside and an increase in humidity.

Figure 5.17 illustrates the data collected outside the apartment (balcony) during the wintertime.

- Outside - As can be seen in Figure 5.17, the temperature and humidity sensors detected dropped in temperature and increased in relative humidity when the remote station was placed outside.

* The outside temperature dropped to -4 degrees Celsius when the developed system was placed outside to collect data.

* The outside humidity increased to $77 \%$ relative humidity at -4 degrees Celsius (event ('G')).

* It was pitch black, there was no light (event ('D')) present outside at the time of collecting the data.

- Inside (living room) - As can be seen in Figure 5.17, as soon the remote station was brought inside the apartment, the temperature and humidity sensors showed the temperature increased gradually while the humidity reading decreased simultaneously. Furthermore, there was an indication of the presence of light in the living room.

* The temperature increased to 4 degrees Celsius when the system was placed inside the apartment (event ('E')).

* The humidity sensor detected dropped of $52 \%$ relative humidity at 4 degrees Celsius (event (' $\left.H^{\prime}\right)$ ). 
* There was light (event $\left.\left({ }^{\prime} C^{\prime}\right)\right)$ present inside the apartment at the time of collecting the data for the Figure 5.17.

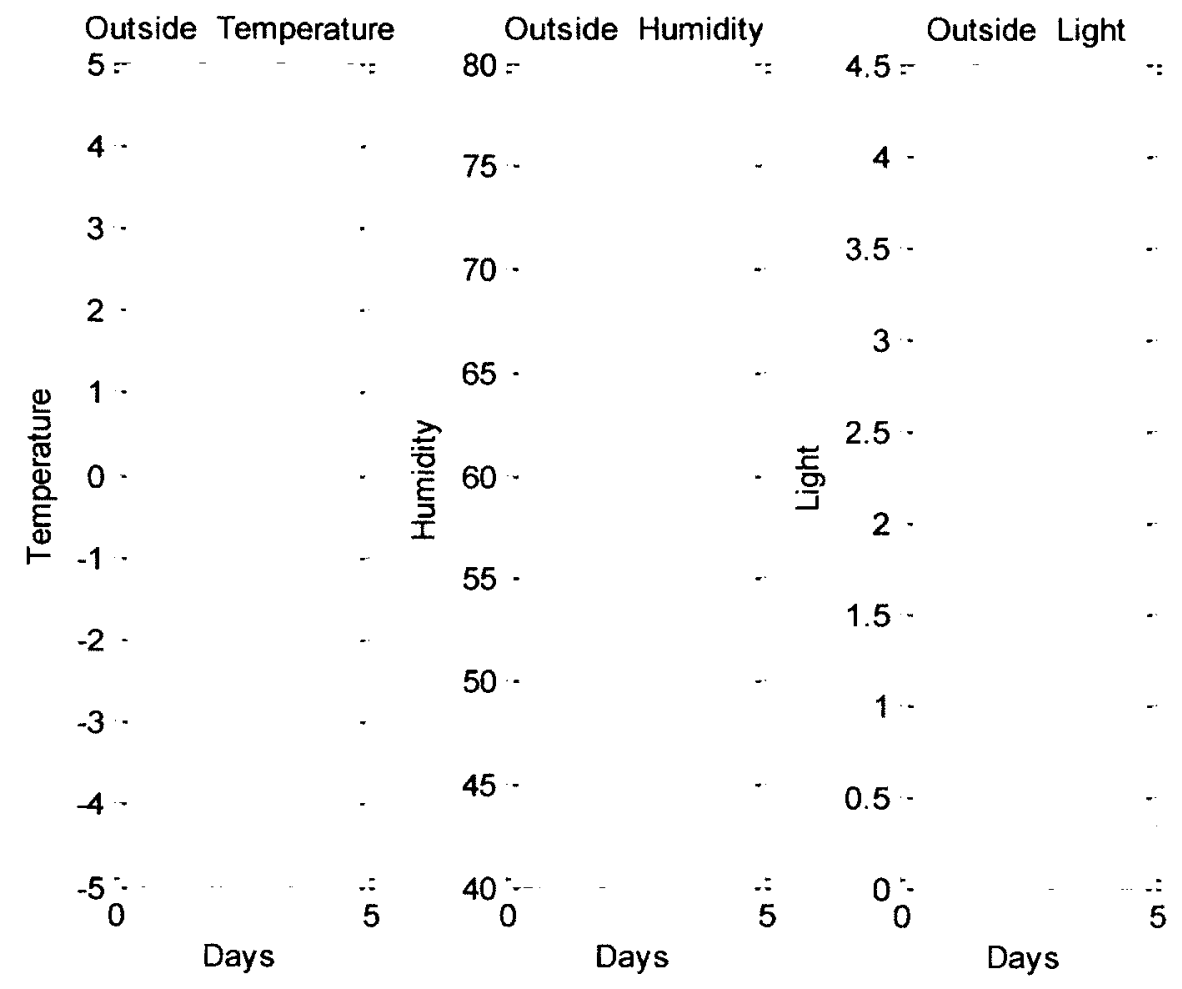

Figure 5.18: Graph of data collected from outside of the apartment

\subsection{Data Interpretations}

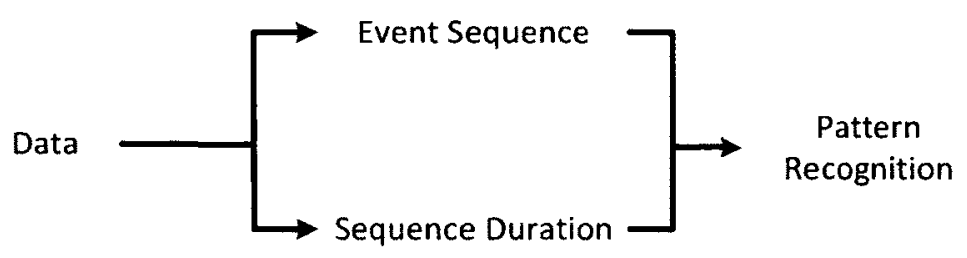

Figure 5.19: Sequence of data interpretations 
Data interpretation is important to identify common patterns such that meaning can be derived. In the data analysis subsection, we assigned a symbol to each event. In this subsection, we will show how to derive meaning from the sequence of these symbols and duration of the events, as shown in Table 5.4. The goal is to potentially identify various Activities of daily living of an occupant from the sequence of the symbols. The Activities of daily living in which we are interested are activities such as bathing, toileting, eating, sleeping, and cooking, as shown in Table 5.4 and Figure 5.19.

\begin{tabular}{|c|c|c|}
\hline $\begin{array}{l}\text { Activities of daily } \\
\text { living (ADL) }\end{array}$ & $\begin{array}{c}\text { Transition state } \\
\text { (sequence) }\end{array}$ & Events \\
\hline Bathing & $A, C, E, G, D, B$ & Motion on, light on, temperature on, humidity on, light off, motion off \\
\hline Toileting & $A, C, B, A, E, G, D, B$ & $\begin{array}{l}\text { Motion on, light on, motion off, motion on, temperature on, humidity } \\
\text { on, light off, motion off }\end{array}$ \\
\hline Cooking & $A, C, I, E, G, J, E, D, B$ & $\begin{array}{c}\text { Motion on, light on, magnetic on, temperature on, humidity on, light } \\
\text { on, magnetic off, temperature on, light off, motion off }\end{array}$ \\
\hline Eating & $D, B, A, C, D, B A, C$ & $\begin{array}{l}\text { Light off, motion off, motion on, light on, light off, motion off, motion } \\
\qquad \text { on, light on }\end{array}$ \\
\hline Sleeping & $A, C, D, B, A, C$ & Motion on, light on, light off, motion off, motion on, light on \\
\hline
\end{tabular}

Table 5.4: Sequence of events of Activities of daily living of an occupant 


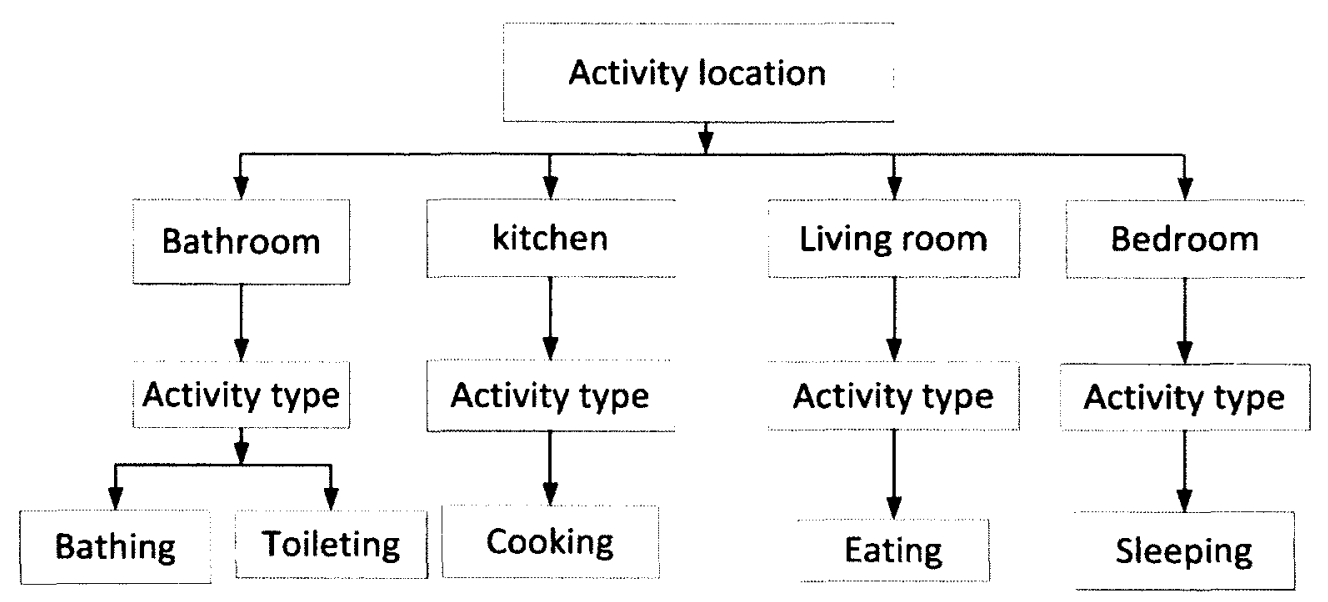

Figure 5.20: Activities of daily living of an occupant

- Possible scenario for bathing activity is as shown in Figure 5.20: An occupant enters the washroom, triggering the motion sensor (event $\left.\left(A^{\prime}\right)\right)$; turns on the light, which activates the light sensor (event $\left.\left({ }^{\prime}\right)\right)$; and proceeds to taking a shower, turning on the hot water tap, which triggers the temperature and humidity to rise (event ('E') and event ('G'), respectively). After the shower, the occupant gets out of the bathtub, takes a towel to rub water off her/his body, puts on some body lotion, dresses, and then moves toward the door to exit the washroom; these activities continue to generate motion (event (' $A^{\prime}$ )). The occupant then turns the light off (event $\left.\left({ }^{\prime} D\right)\right)$ and departs the washroom (event ('B')). The expected duration of the activity is 10-15 minutes as shown in Figure 5.20. 


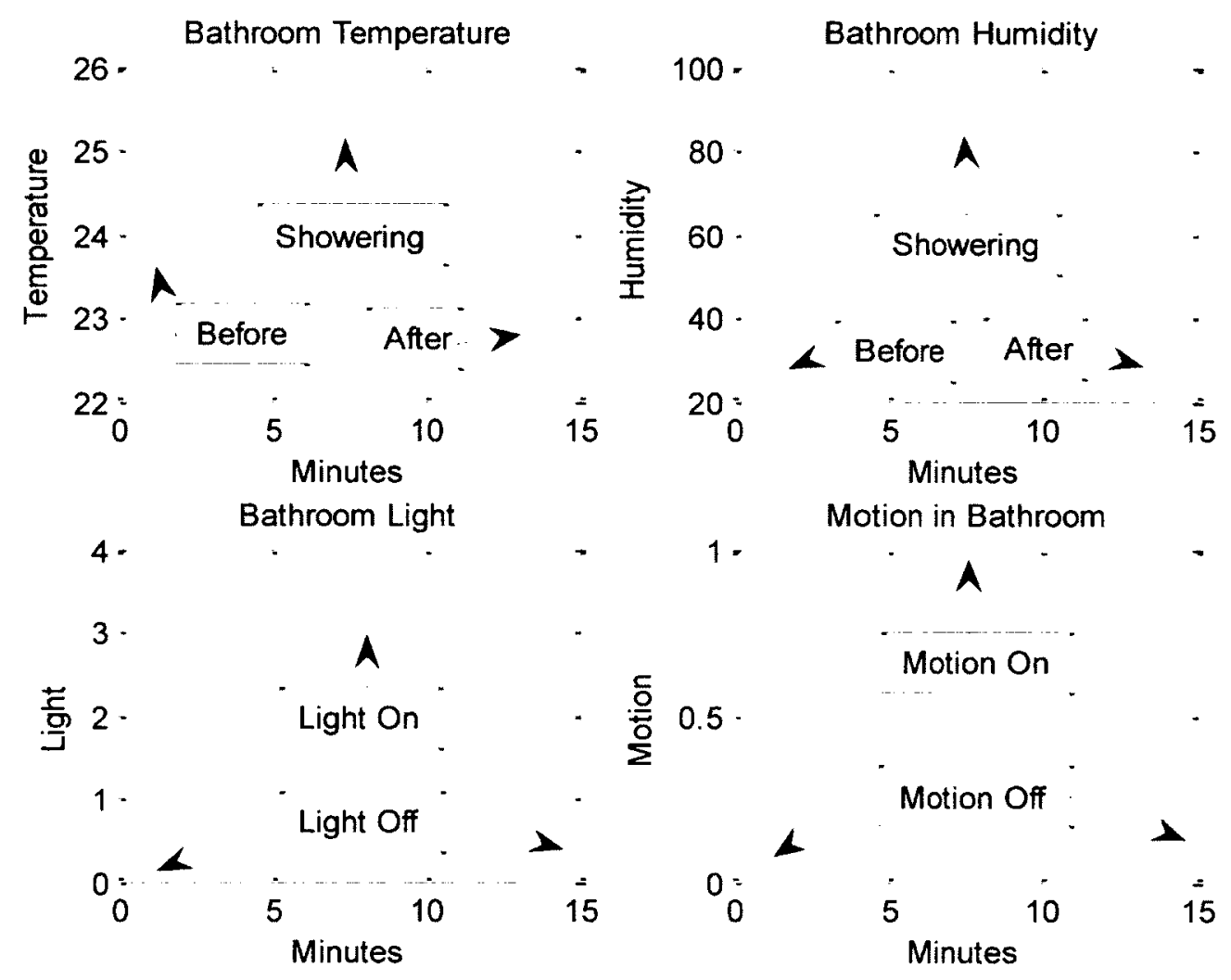

Figure 5.21: Bathroom results

- Possible scenario for toileting activity is as shown in Figure 5.21: : An occupant enters the washroom, triggering the motion sensor (event ('A')); turns on the light (event $\left({ }^{\prime} C^{\prime}\right)$; and sits down on toilet seat, where they make no movement at all and therefore trigger the motion off (event $\left({ }^{\prime} B^{\prime}\right)$ ). The occupant moves to stand up, triggering the motion sensor on (event ('A')); flushes the toilet; and turns on the tap to wash their hands, triggering the temperature and humidity to rise (event ('E') and event ('G'), 
respectively). The occupant then moves toward the door and turns off the light (event $\left.\left({ }^{\prime} D^{\prime}\right)\right)$ and departs (event $\left.\left({ }^{\prime} B^{\prime}\right)\right)$. The expected duration of the event is 5-10 minutes as shown in Figure 5.21.

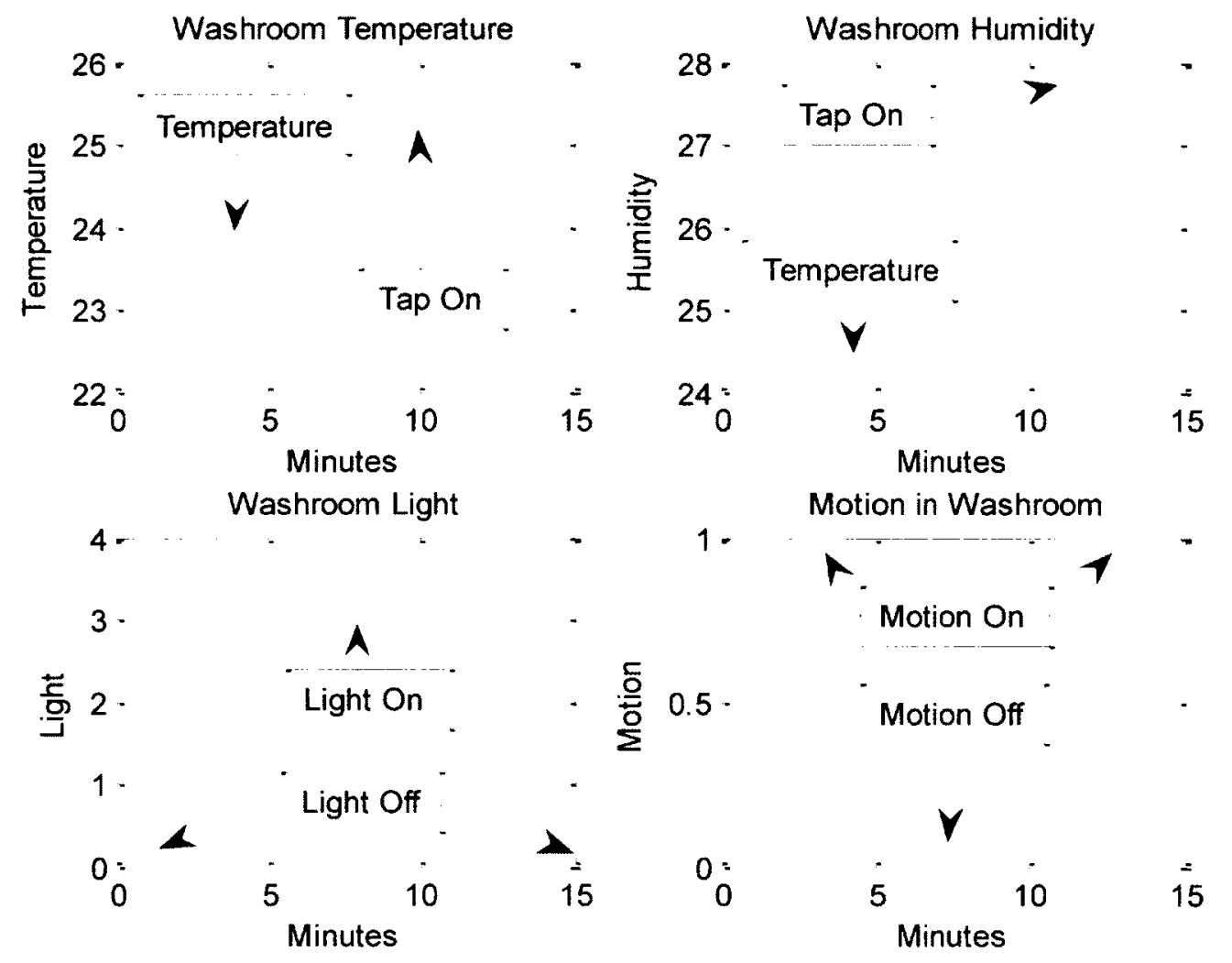

Figure 5.22: Washroom results

- Possible scenario for cooking activity is as shown in Figure 5.22: An occupant enters the kitchen, triggering the motion sensor (event ('A')), and switches on the light (event $\left({ }^{\prime} C^{\prime}\right)$ ). The occupant then moves toward the refrigerator and opens the refrigerator door, triggering the magnetic sensor on (event $\left.\left({ }^{\prime} l^{\prime}\right)\right)$. The refrigerator's light turns on, as it does automatically whenever the refrigerator door is opened (event $\left({ }^{\prime} C\right.$ ')), and the temperature and humidity rise (event (' $E$ ') and event (' $G$ '), respectively) as shown 
in Figure 5.13. The occupant then closes the fridge door, triggering the magnetic sensor off, (event (' $\mathrm{J}$ ')) and refrigerator's light turns off, as it does automatically whenever the refrigerator door is closed (event $\left.\left(' D^{\prime}\right)\right)$. The occupant switches the stove on, prompting the temperature sensor to go up (event ('E')). The occupant's movement and activity around the kitchen continues to trigger the motion sensor on (event $(' A '))$ in the kitchen. Upon completion of food preparation, the occupant turns off the light (event ('D')) and departs from the kitchen, triggering the motion sensor to not detect any motion (event (' $\left.\mathrm{B}^{\prime}\right)$ ) in the kitchen. The expected duration of the cooking event is 10-30 minutes as shown in Figure 5.22.

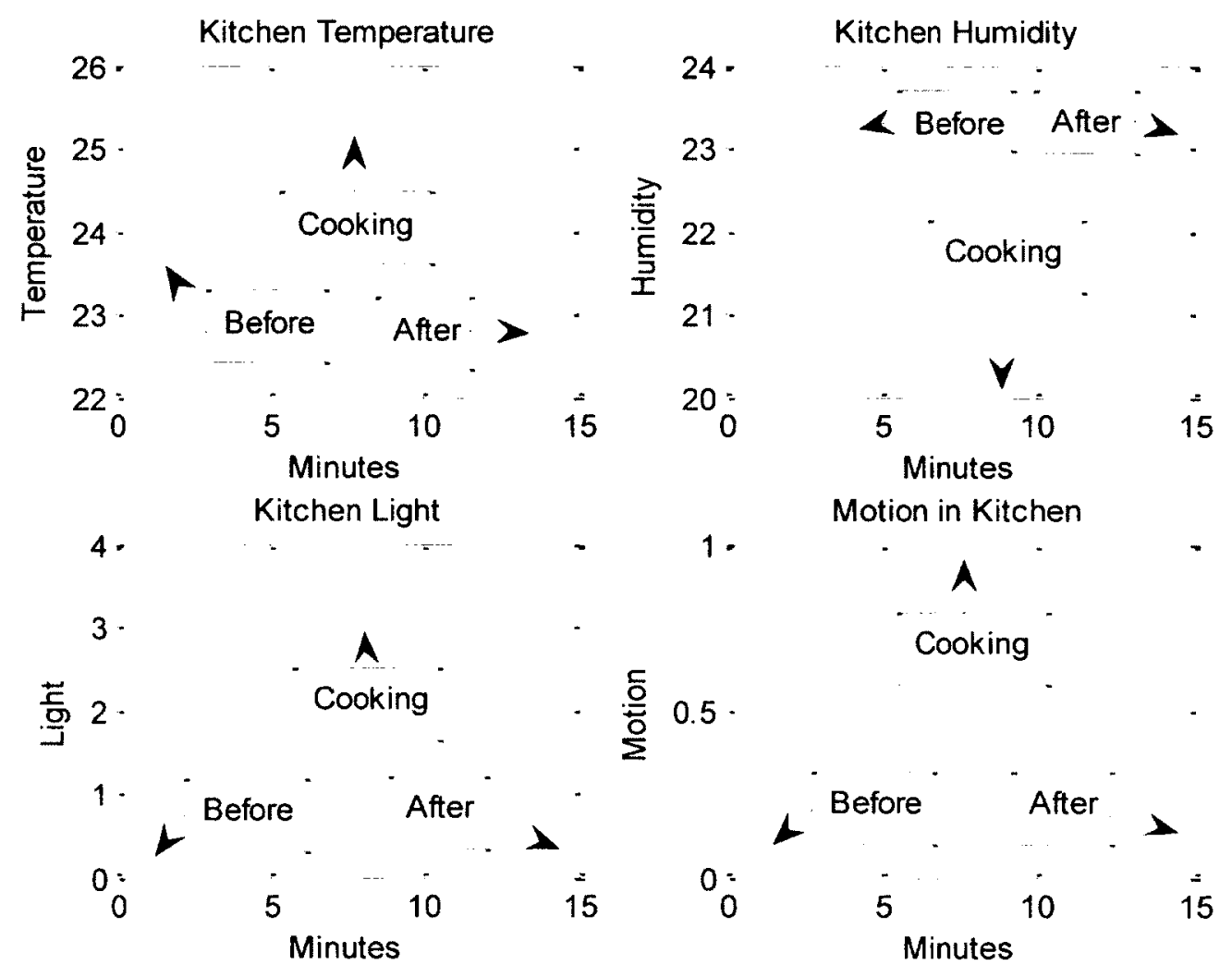

Figure 5.23: Kitchen results 
- Possible scenario eating activity is as shown in Figure 5.23: The activity starts in the kitchen, moves to the living room, and then back to the kitchen again. Upon completion of food preparation, the occupant turns off the kitchen light (event $\left.\left({ }^{\prime} D^{\prime}\right)\right)$ and departs from the kitchen, triggering the motion sensor to off (event $\left.\left({ }^{\prime} B^{\prime}\right)\right)$. The occupant then enters the living room, triggering the motion sensor (event (' $\left.\left.A^{\prime}\right)\right)$, turns on the light, (event $\left({ }^{\prime} C^{\prime}\right)$ ), and sits down to eat. After the meal the occupant goes back to kitchen, triggering the motion sensor on, (event $\left.\left({ }^{\prime} A '\right)\right)$, and switches the kitchen light on again (event (' $C$ ')). The expected duration for the eating events is 10-30 minutes as shown in Figure 5.23.

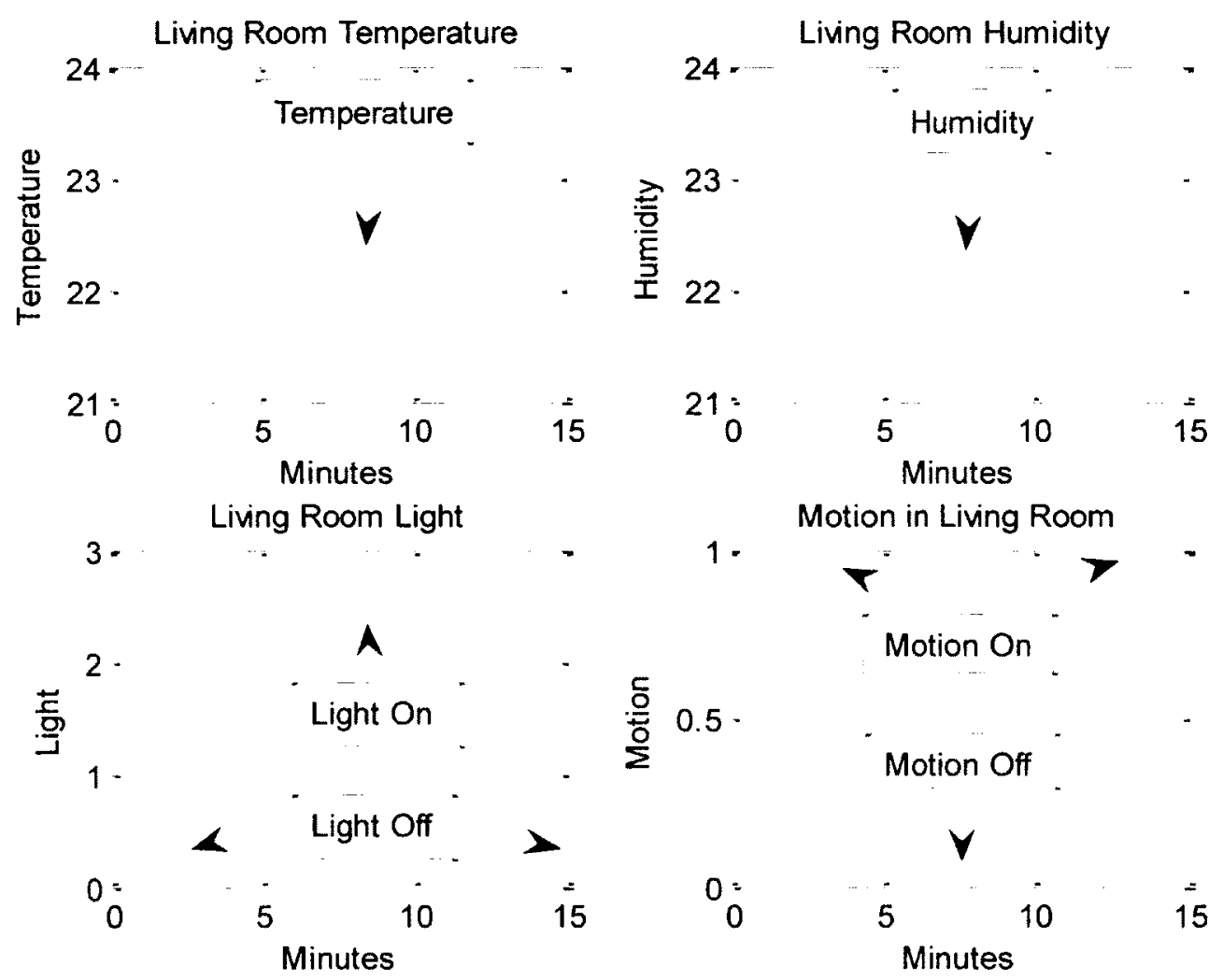

Figure 5.24: Living Room results 
- Possible scenario for sleeping activity is as shown in Figure 5.24: The activity starts at the time that the occupant goes to bed and ends at the time that the occupant wakes up. The occupant enters the bedroom, triggering the motion sensor (event (' $\left.A^{\prime}\right)$ ); turns on the lamp (event (' $C$ ')); and proceeds to the bed to lay down on it. The occupant then turns the lamp off (event $\left.\left({ }^{\prime} D^{\prime}\right)\right)$ and lays down on bed to sleep, triggering the motion sensor off (event $\left.\left({ }^{\prime} B^{\prime}\right)\right)$. The occupant wakes up in the morning, triggering the motion sensor on (event $\left({ }^{\prime} A\right)$ ). The expected duration of sleep is 6-8 hours as shown in Figure 5.24.

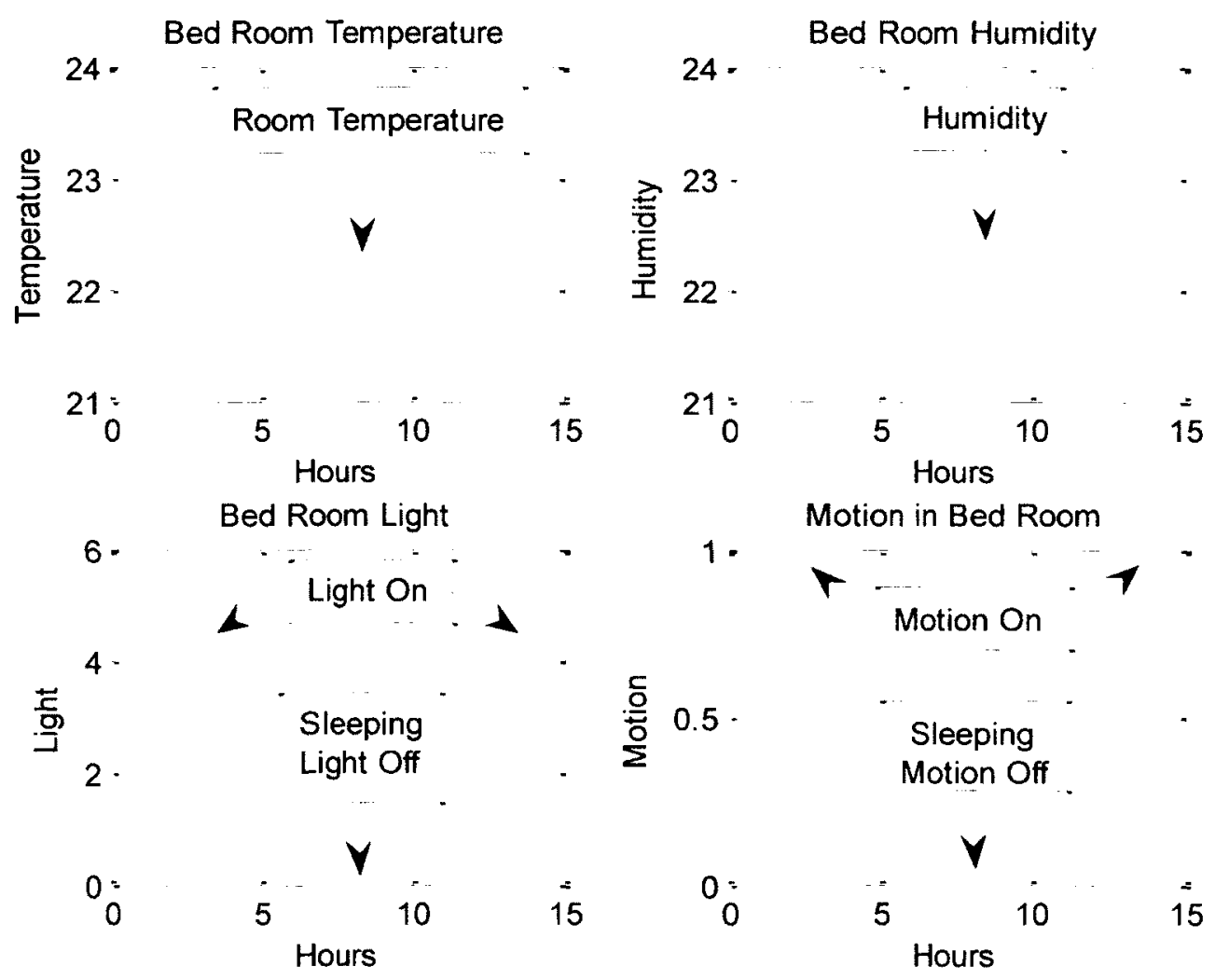

Figure 5.25: Bed Room results 


\subsection{Potential Benefits of the Research}

As we have seen, the monitoring system can automatically detect the Activities of daily living (ADL) of elderly populations in their home to improve the occupant's health and well-being, and the safety of their living conditions. This section will describe some of the potential benefits of our developed system.

- Health and well-being: Health and well-being can be determined through the level of mobility and the performance of ADL of the occupant. Early signs of health problems of the occupant can be identified from abnormal patterns in their ADL. Moreover, knowing if sleeping, eating, or bathing times change day after day is also a good indication of health problem. The objective here is early recognition of abnormal conditions and prompt, appropriate, and cost-effective interventions of medical and welfare professionals to help reduce morbidity and maintain independent lifestyle of the elderly in their own home.

- Food spoilage: The refrigerator is one of most important appliances in the kitchen in regards to keeping food safe. It helps protect food from spoiling by slowing bacterial growth. Bacteria grow rapidly at temperatures between $4^{\circ} \mathrm{C}$ and $60^{\circ} \mathrm{C}$. This temperature range is commonly referred to as the "Danger Zone." The refrigerator temperature should be $4^{\circ} \mathrm{C}$ or lower. This temperature level and refrigerator door should always be monitored to prevent food from spoiling.

- Well-cooked food: It is important to cook food thoroughly to kill harmful bacteria that might contaminate food, especially meat. Sometimes meats are contaminated with pathogens ${ }^{17}$; to kill these germs, meat must be cooked thoroughly at a temperature of at least $77^{\circ} \mathrm{C}$. However, high temperatures destroy certain vitamins and minerals in food, so food must be cooked at the temperature range that is

\footnotetext{
${ }^{17}$ Pathogens are germs that cause serious illness.
} 
suitable for each type of food. Our designed prototype system is suitable for monitoring food temperature levels to ensure safety.

- Negligence: Caregiver negligence is one of the problems facing the elderly population. One example of negligence is the well-known story in June 2010 of the elderly person in Toronto who was neglected for several months in a makeshift bedroom setup inside an uninsulated and inadequately heated garage. She was left there with only a bucket of water, very little food, and a port-a-potty that was not properly maintained [63]. She was found unconscious, starving, and suffering from frostbite. Monitoring systems such as ours that can automatically detect the daily living activities of elderly populations in their home can lead to the improvement of their health and well-being, and the safety of their living conditions and could have made a difference in this situation. Another story of neglect is that of an elderly couple in the UK, Mr. Randall, 76, and his wife Mrs. Jean, 79 [64]. The couple was left to die in their freezing home after neighbours' pleas for help to the authorities were ignored. The system that can monitor room temperature and make sure it does not drop below ambient temperature, especially in the winter, could have saved the couple.

- Intruders: Another issue facing elderly people is that of intruders. There are frequently reports of breakins into the homes of the elderly and assaults of elderly people that could be avoided. One way of preventing these crimes is by continuously monitoring windows and main entrances of buildings to ensure they are locked at all times as well as putting in place a system with a buzzer that would send out an alert if doors and windows are open for a long period.

- Water leakage: There are so many headline news stories of water leakage problem facing elderly. The stories such as "Water heater's monoxide leak kills elderly couple." Taipei City police found the bodies of 
an elderly couple living in the Shizhi District who had reportedly died from carbon monoxide poisoning resulting from an overheated water heater in a tightly sealed home [65]. A continually monitoring of home temperature and humidity levels to avoid water leakage or water damage restoration.

- Energy consumption: Energy consumption is one of the most pressing issues facing the elderly population. Sometime elderly people forget to switch off the stove or turn off the lights, faucets, or tap after usage, which leads to heat loss and energy waste. One way of controlling this is through continuously monitoring the major domestic equipment: the hot water heater, stove, refrigerator, and light switches. This can detect the usage pattern and the impact of seasonal difference on energy usage and help identify when a particular appliance is $\mathrm{ON}$ and the length of time that it has been $\mathrm{ON}$.

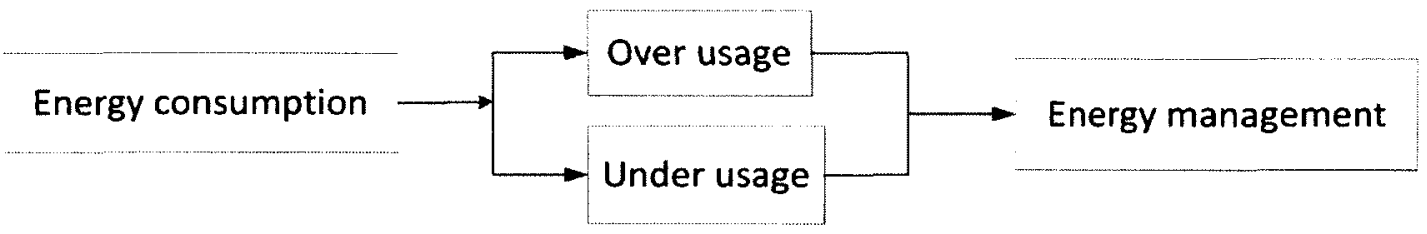

Figure 5.26: Energy consumption due to activities of daily living of an occupant

\subsection{Potential Benefits of Sensors}

The experimental results for our collected data at different locations and environments clearly show that our prototype system can achieve high levels of accuracy. Each sensor in the prototype system has the potential of benefitting the occupant in the smart apartment. The potential goals are discussed as follows.

- Temperature sensor: The goal of the temperature sensor is to monitor environmental temperature to ensure appropriate heating and cooling of the apartment. Also, the aim of the temperature sensor is to monitor the condition of appliances in the apartment, such as the refrigerator or stove, to ensure they 
are operating at an acceptable temperature range. The designed system also includes a buzzer to alert the user of any change in the normally required range of temperature in the apartment or appliances.

- Photocell sensor: The goal of the photocell sensor is to monitor the presence of light in the apartment. This information could potential help an occupant turn the light off when light is not supposed to be on. Another goal of the light sensor is to monitor the condition of appliances, such as the fridge. From the experimental results of the fridge, we have learned that whenever the fridge door is open, the fridge light turns on automatically. As soon as the door is closed, the fridge light turns off. With this information, it can be easily recognized when the fridge door is open or closed; this can lead to ensuring that the fridge door is closed to prevent food from spoiling.

- Pyroelectric Infrared (PIR) Motion Sensor: The goal of the sensor is to monitor the presence and motion of the resident throughout the apartment. Information about the occupant's health, hygiene, and eating patterns can be determined from the motion sensor. For example,

(1) Concerns about the occupant's hygiene can be determined from motion in the bathroom.

(2) Concerns about the eating behaviour of the occupant can be determined from motion in the kitchen and living room.

(3) Health concerns about the occupant can be determined from prolonged presence of motion in one place or room and no motion throughout the entire apartment. The information here can indicate that occupant might be too ill to move around the apartment. Also, irrational 
wondering or random changes of motion in the apartment could indicate mental anxiety or confusion.

- Humidity sensor: The aim of the humidity sensor is to monitor environmental relative humidity of the apartment or appliances to enable the observer to determine the Activities of daily living of the user and the condition of their appliances in the apartment. From the experimental results of the fridge, we have detected that when the fridge door is open, the relative humidity of the fridge increases. We also observed an increase in relative humidity when the bathtub is filled with water and when windows or the balcony door is open. With this information, the following information can be determined:

(1) The occupant taking a shower can be determined from changing levels of relative humidity in the bathroom.

(2) The fridge functionality can be determined from the relative humidity of the fridge.

(3) An open balcony door or window can be determined by changes in the relative humidity of the apartment.

- Ultrasonic sensor: The objective of the ultrasonic sensor is to detect an object and measure the discrete distances between objects in the apartment. For example, it can be used to measure a distance between an object in the kitchen or bathroom of the apartment; this information can identify the exact location of an occupant in the apartment.

- Hall Effect (magnetic) sensor: The purpose of the Hall Effect sensor is to detect whether a door is open or closed. It is convenient for the monitoring of the entry and exit from various rooms or the apartment. 
It is also useful for monitoring windows or kitchen appliances, such as the refrigerator, dishwasher, or stove.

- Piezoelectronic buzzers: There are also buzzers that will alert the users of any unacceptable changes in behaviour. An example of an unacceptable change in behaviour would be when the temperature of the apartment or appliances, such as fridge, is lower or higher than the acceptable range.

The primary goal of the research is the early recognition of abnormal conditions such that automatic, prompt, appropriate, and cost-effective interventions can be implemented by medical and welfare professionals to help reduce the morbidity of elderly people and maintain independent lifestyles of this population in their own homes. The target intervention will potentially help reduce requests for expensive medical services and reduce elderly demands in emergency. 


\section{CHAPTER 6}

\section{CONCLUSION, LIMITATIONS, AND SUGGESTIONS FOR FUTURE RESEARCH}

This chapter is organized into two sections: section 6.1 focuses on the conclusions of the research and section 6.2 emphasizes limitations and suggestions for future research.

\subsection{Conclusions}

The objective of the research was achieved. We designed, developed, and implemented an ultra-safe and reliable wireless sensor network system with multiple functions that functions trouble-free in different environments and require minimal maintenance. The system is cost-effective, easy to use, portable, and consumes less power than other wireless sensor networks, such as Wi-Fi, Bluetooth, wireless USB, Ultra wideband (UWB), or IR wireless. Nowadays, with busy lifestyles, a wireless sensor network is an excellent device to monitor vulnerable, isolated, or neglected people in society, such as the many senior citizens who are left alone in their homes with little or no supervision. With this contribution, this research could potentially lead to major cost savings in regards to the monitoring of the elderly population in that they can maintain an independent, healthy lifestyle in their own home rather than relocate to more expensive and isolated care facilities. However, it is up to the users to decide whether to accept this wireless device as an integral part of their life. 


\subsection{Limitations and Future Research}

\subsubsection{Limitations}

Although the research has reached its objectives, there were some limitations to the research. Some of these limitations will be discussed in this section.

Data retrieval: The developed system only collects data and does not store it for future research. We suggest that future research further develop this system to include storage features that store data for future studies.

Design and implement control features: The developed system we proposed monitors the activities of daily living but does not control the activities. For future research, we recommend that the design system include control features.

Multiple remote stations: Although the developed system we proposed can be used for collecting data from multiple remote stations, we were unable to receive data from multiple remote stations simultaneously, instead we used pair form. Pair form is the simplest network with just two radios, a coordinator, and one remote station. To further the impact of this research, we suggest future research look into these issues.

Algorithm and implementations: The developed system we proposed for monitoring does not include algorithms for pattern recognition; we suggest this be included in future research. Some of the most common machine learning algorithmic models used in this area are Bayesian Network, Logic Models, Decision Tree, Artificial Networks, and Dynamic Bayesian models. 


\subsubsection{Suggestion for Future Research}

Although we have reached our goals, there are ways that the research can be improved. Some recommendations that we suggest for future research are described in this section.

Extend the range of data transmissions: The developed system we proposed transmits data up to the range of 133 feet ( 40 meters) indoors and 400 feet ( 120 meters) outdoors at Line-of-sight. We suggest the range of transmission should be extended for future research such that data can be collected at as great a distance as possible.

Power consumption: We used electric outlets as the power source for our system since it was readily available at the area of data collection, as such, we do not know the exact life expectancy of the system when using batteries. For future research, if the system is going to be used with batteries as the power source to collect data indoors or outdoors, then the life expectancy of the battery should be determined.

Incorporate new type of sensors in the environment: New type of sensors may help to recognize sets of more complex activities, or activities not detected before. For example, a sensor which is able to monitor current consumption of electric/electronic appliances is likely to provide powerful hints about device usability [66]. Other sensors that can be considered such as a water-flow sensor to monitor water flow and a force sensor which can be installed in the bed, chair, couch, or mat to pinpoint the exact location of the occupant when the occupant is cooking, eating, or sleeping.

Publication of the research: One of the most important parts of the research that is not yet completed is publication of the results of the research. It will be ideal to consider the research for publication in future. 
131

Cellular, WiMAX, WiFi, or Ethernet connection: For future research, we recommend the use of ConnectPort $X$ shown in Figure 6.1. ConnectPort $X$ is a gateway that provides PC or IP Network connectivity for end point devices in ABe WPANs, allowing data to be collected anywhere in the world. Gateways collect end node data, aggregate it, and send it to a parent application using cellular, WiMAX, WiFi, or Ethernet connections. ConnectPort $X$ gateway can extend wireless connectivity to other devices such as Smart mobile phones.
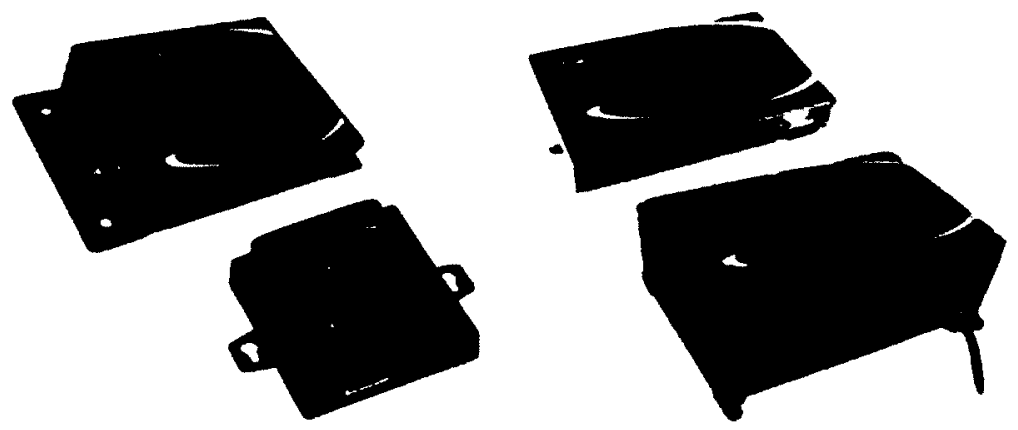

Figure 6.1: Different types of ConnectPort $X$ gateway 


\section{REFERENCES}

[1] A. Tinker and C. McCreedie in "The Application of Research in Technology and Design to Improve the Quality of Life of Older People" Ageing: Scientific Aspects $1^{\text {st }}$ ed., Stationery Office Books, Vol. 2, Jan. 2005, pp. 119 - 120.

[2] L. Jiang, D.Y. Liu, and B. Yang, "Smart Home Research," Proc. The International Conference on Machine Learning and Cybernetics, Shanghai, China. Aug. 2004, pp. $659-663$.

[3] J. Choi; D. Shin and D. Shin, "Research on Design and Implementation of the Artificial Intelligent Agent for Smart Home Based on Support Vector Machine," First International Conference, ICNC 2005, Changsha, China, August 27-29, 2005, pp.1185-1188.

[4] M. Chan, D. Esteve, C. Escriba, and E. Campo, "A Reviews of Smart home-Present state and future challenges," Computer Methods and Programs in Biomedicine, vol. 91, pp.55-81, Feb. 2008.

[5] B. Seung-Ho, P. Jae-Han, J. Koh, P. Kyung-Wook, and B. Moon-Hong, "Building a Smart Home Environment for service Robots Based on RFID and sensor Networks" Proc. The International Conference on control, Automation and System 2007, Seoul, Korea, Oct. 17-20, 2007, pp. 1078 1082.

[6] P. Rashidi, and D. Cook, "Keeping the resident in the loop: adapting the smart home to the user," IEEE trans. Syst. Man, Cybern. A, Syst., Humans, vol.39, no. 5, pp. 949-959, Sep. 2009.

[7] S. Junnila, H. Kailanto, J. Merilahti. A. Vainio, A. Vehkaoja, M. Zakrzewki, and J. Hyttinen, "Wireless, multipurpose in-home health platform: two case trials, "IEEE trans. Inf. Technol. Biomed., vol. 14, no. 2, pp. 447-455, Mar. 2010.

[8] P. Carner. "Project Domus: Designing Effective Smart Home System." Academic Project, School of Computing, Faculty of Science, Dublin Institute of Technology, Dublin, Ireland, 2009.

[9] J. Cheng and T. Kunz "Smart home networking: Combining wireless and powerline networking" Proc. 2011 7th International Conference on Wireless Communications and Mobile Computing (IWCMC), July 4-8, 2011, pp.1276- 1281 .

[10] J. Cheng and T. Kunz, "A Survey on Smart Home Networking," Dept. Sys.Comp., Eng., Carleton Univ., Ottawa, Ont., Canada, Tech. Rep. SCE-09-10, Sep. 2009. 
[11] D. Townsend, M. Holzman, R. A. Goubran, M. Frize, and F. Knoefel, "Relative Thresholding with Under Mattress Pressure Sensors to Detect Central Apnoea", IEEE Transactions on Instrumentation and Measurement, vol. 60, no. 10, Oct. 2011, pp. 3281-3289.

[12] D. Townsend, R. A. Goubran, and F. Knoefel, "Amplitude-Based Central Apnea Screening," in proc. IEEE International Symposium on Medical Measurements and Applications proceesing (MeMeA), Bari, Italy, May 30-31, 2011, pp. 395-398

[13] D. Townsend, M. Holtzman, R. A. Goubran, M. Frize, and F. Knoefel, "Effect of Windowing on Central Apnea Detection", proc. IEEE International Workshop on Medical Measurements and Applications (MeMeA), Ottawa, Canada, April 30 2010-May 1 2010, pp. 117 - 120.

[14] D. Townsend, R.A. Goubran, M. Frize, and F. Knoefel, "Effect of Sensor Position on Unobtrusive Rollover Detection for Long-Term Sleep Monitoring in Smart Homes", Proc. 31st Annual International Conference on the IEEE Engineering in Medicine and Biology Society, (EMBS'O9), Minneapolis, Minnesota, USA, September 2-6, 2009, pp. 6135-6138.

[15] F. Knoefel, R.A. Goubran, and M. H. Jones, "Using a Bed Sensor Array to Monitor Sleep", Annual Scientific Meeting of the Canadian Geriatric Society, Banff, Alberta, Canada, Vol. 10, Issue 1, pp. 3334, April 2007.

[16] M. Holzman, D. Townsend, R. A. Goubran, and F. Knoefel, "Breathing Sensor Selection During Movement", Proc. 33rd Annual International Conference of the IEEE Engineering in Medicine and Biology (EMBC'11), Boston, MA, U.S.A., Aug. 30 2011-Sept. 3 2011, pp. 381 - 384.

[17] D. Townsend, R. A. Goubran, M. Frize, and F. Knoefel, "Measuring Chest Movement with an Array of Unobtrusive Pressure Sensors", proc. IEEE International Instrumentation and Measurement Technology Conference (I2MTC), Austin Texas, May 2010, pp. $1053-1056$. 
[18] A. Arcelus, R. A. Goubran, F. Knoefel, H. Sveistrup, and M. Bilodeau, "Detection of Bouncing During Sit-to-stand Transfers with Sequential Pressure Images," proc. IEEE International Symposium on Medical Measurements and Applications, Bari, Italy, May 2011, pp. 158-161.

[19] A. Arcelus, I. Veledar, R. Goubran, F. Knoefel, H. Sveistrup, and M. Bilodeau, "Measurements of Sit-to-Stand Timing and Symmetry from Bed Pressure Sensors", IEEE Transactions on Instrumentation and Measurement, vol. 60, no. 5, May 2011, pp. 1732-1740.

[20] I. Veledar, A. Arcelus, R. Goubran, F. Knoefel, H. Sveistrup, and M. Bilodeau, "Sit-to-stand Timing Measurements using Pressure Sensitive Technology", in proc. IEEE International Instrumentation and Measurement Technology Conference (I2MTC), Austin Texas, May 2010, pp. 1337 - 1340.

[21] J. Nikolic-Popovic, and R. A. Goubran, "Measuring Heart Rate, Breathing Rate and Skin Conductance During Exercise," in proc. IEEE International Symposium on Medical Measurements and Applications, Bari, Italy, May 2011, pp. 507-511.

[22] M. Holtzman, R. A. Goubran, and F. Knoefel, "Maximal Ratio Combining for Respiratory Effort Extraction from Pressure Sensor Arrays", in proc. IEEE International Symposium on Medical Measurements and Applications, Bari, Italy, May 2011, pp. 88-92.

[23] X. He, R. A. Goubran, and P. Liu, "Signal Enhancement of Wearable ECG Monitoring Sensors Based on Ensemble Empirical Mode Decomposition," in proc. IEEE International Symposium on Medical Measurements and Applications, Bari, Italy, May 2011, pp. 433-436.

[24] G. Green, A. Chan, and R. A. Goubran, "Monitoring of Food Spoilage with Electronic Nose: Potential Applications for Smart Homes", in Proc. 3rd International Conference on Pervasive Computing Technologies for Healthcare, Pervasive Health 2009, London, U.K., April 2009, pp. 1-7. on Neural and Fuzzy Processing of Odour Sensor Responses", in Proc. International Conference of 
the IEEE Engineering in Medicine and Biology Society (EMBC'09), Minneapolis, Minnesota, USA, September 2009, pp. 2626-2628.

[26] A. Arcelus, M. H. Jones, R. A. Goubran, Frank Knoefel, "Integration of Smart Home Technologies in a Health Monitoring System for the Elderly", IEEE First International Workshop on Smart Homes for Tele-Health, Smartel 2007, Niagara Falls, Canada, May 2007, pp. $820-825$.

[27] A. Arcelus, R. A. Goubran, H. Sveistrup, M. Bilodeau, and F. Knoefel, "Context-Aware Smart Home Monitoring Through Pressure Measurement Sequences, in proc. IEEE International Workshop on Medical Measurements and Applications (MeMeA), Ottawa, Canada, April 2010, pp. 32 - 37.

[28] M. Holtzman, D. Townsend, R.A. Goubran, and F. Knoefel, "Validation of Pressure Sensors for Physiological Monitoring in Home Environments", in proc. IEEE International Workshop on Medical Measurements and Applications (MeMeA), Ottawa, Canada, April 2010, pp. 38 - 42.

[29] A. Arcelus, M. Holtzman, R. A. Goubran, H. Sveistrup, P. Guitard, and F. Knoefel, "Analysis of Commode Grab Bar Usage for the Monitoring of Older Adults in the Smart Home Environment", in Proc. International Conference of the IEEE Engineering in Medicine and Biology Society, (EMBC'09), Minneapolis, Minnesota, USA, September 2009, pp. 6155-6158

[30] P. Carlson, N. Foubert, F. Knoefel, R. A. Goubran, M. Bilodeau and H. Sveistrup, "Smart Mat Technology: Can it Differentiate Bed Transfers in Young Healthy, Old Healthy and Old Stroke Populations", Canadian Geriatrics Society Annual General Meeting, 2009, Toronto, Canada, April 2009.

[31] A. Arcelus, M. Holtzman, I. Veledar, R.A. Goubran, H. Sveistrup, and P. Guitard, "Contact Location Estimation from a Nonlinear Array of Pressure Sensors," in Proc. of the IEEE International Instrumentation and Measurement Technology Conference, I2MTC 2008, Victoria, B.C., May 2008, pp.1969-1973.

[32] TAFETA. Internet: http://www.tafeta.ca. 
[33] A. Arcelus, "Context- Aware Smart Home Monitoring Through the Analysis of Presure Sequences", Ph.D. dissertation, Dept. Sys. and Comp. Eng., Carleton University, Ottawa, ON, Canada, 2010.

[34] P. L. Divya, R. V. Kulkarni, and R. Manoj, "A Swarm Intelligence based distributed localization technique for Wireless Sensor Network," International Conference on Advances in Computing, Communications and Informatics (ICACCI-2012), pp. 367 - 373, 2012.

[35] G. Vijayalakshmi. and D. Tamilarasi, “Deploying Storage Node Aiming to Minimize the Communication Cost of Sink in Sensor Networks," International Journal of Computer Network and Security(IJCNS), Vol. 4, No. 1, pp. 42-47, Jan-Mar 2012.

[36]

Digi International, "Wireless Sensor Networking", Internet: http://www.digi.com/technology/drop-in-networking/wireless-sensor-networking. 2012, [Dec. 05, 2012].

[37] Digi international, "Wireless Mesh Networking ZigBee ${ }^{\star}$ vs. DigiMesh," white paper, Internet: http://www.digi.com/pdf/wp zigbeevsdigimesh.pdf, 2008, [Dec. 5, 2012].

[38] F. R. Robles and P. Kaminski, "Generating acknowledgement signals in a data communication system," Patent number: 6282172, Aug 28, 2001.

[39] U. Agarwal, "Wireless Sensor Networks - An Emerging Technology" Computers and Technology: Mobile Computing" Avalailable: http://ezinearticles.com/?Wireless-Sensor-Networks---AnEmerging-Technology\&id=1374627, Aug. 6, 2008, [Dec. 5, 2012].

[40] D. Malan, T. F. Jones, M. Welsh, and S. Moulton, (2004) "CodeBlue: An Ad Hoc Sensor Network Infrastructure for Emergency Medical Care" Division of Engineering and Applied Sciences, Harvard 
University, available: http://www.eecs.harvard.edu/ mdw/proj/codeblue/ and Available: http://www.eecs.harvard.edu/ mdw/papers/codeblue-bsn04.pdf, [Oct. 25, 2011].

[41] R. A. Rashid, M. R. Abd Rahim, S. H. Syed Arifin, S.K. Syed Yusof, and N. Fisal "Wireless Biomedical Sensor Network for Home-based ECG Monitoring," Int. J. Biomedical Engineering and Technology, pp. , 2008.

[42] C.R. Baker; K.Armijo; S. Belka; M. Benhabib; V. Bhargava; N. Burkhart; A. Der Minassians; G. Dervisoglu; L. Gutnik; M.B. Haick; C. Ho; M. Koplow; J. Mangold; S. Robinson; M. Rosa; M. Schwartz; C. Sims; H. Stoffregen; A. Waterbury; E.S. Leland; T. Pering; and P.K. Wright; "Wireless Sensor Networks for Home Health Care" IEEE 21st International Conference on Advanced Information Networking and Applications Workshops (AINAW'07), Aug. 2007, PP. 832 - 837.

[43] V. Boonsawat, J. Ekchamanonta, K. Bumrungkhet, and S.Kittipiyakul , "XBee Wireless Sensor Networks for Temperature Monitoring", Comp. and Com. Tech., Thammasat University, PathumThani, Thailand, Avalaible: http://wenku.baidu.com/view/0dbade10cc7931b765ce15c1.html,

[44] S.Veerasingam, S. Karodi, S.Shukla and M.C.Yeleti; "Design of Wireless Sensor Network Node on ZigBee for Temperature Monitoring" IEEE ACT '09. International Conference on Advances in Computing, Control, \& Telecommunication Technologies, Jan. 2010, PP. $20-23$.

[45] Y.Yussoff; H.Z. Abidin; R.A Rahman; and F.H.Yahaya; "Development of a PIC-based wireless sensor node utilizing XBee technology" IEEE 2nd International Conference on Information Management and Engineering (ICIME), June 2010, PP. 116- 120.

Koh B. K-P and Kong P-Y, "Performance Study on ZigBee-Based Wireless Personal Area Networks for Real-Time Health Monitoring" ETRI Journal, vol. 28, August, 2006. 
[47] V. K. Sehgal, Nitin, D. S. Chauhan, and R. Sharma, "Smart Wireless Temperature Data Logger Using IEEE 802.15.4/ZigBee Protocol," 2008 IEEE Region 10 Conference, 2008, pp. 1 - 6. Available Internet: http://ieeexplore.ieee.org/stamp/stamp.jsp?tp=\&arnumber $=4766744$ [Dec. 05, 2012$].$

[48] K. Manabe, T. Matsui, M. Yamaya, T. Sato-Nakagawa, N. Okamura, H. Ari, and H.Sasaki, Sleeping patterns and mortality among elderly patients in geriatric hospitals, Gerontology, vol. 46 pp. 318$322,2000$.

[49] Y. Sahaf, "Comparing Sensor Modalities for Activity Recognition," M. S., Dept., Elec. Eng. \& Comp. Sci., Washington State University, Washington State, USA, 2011.

[50] I. F. Akyildiz, W. Su, and Y. Sankarasubramaniam, "Wireless sensor networks: a survey," Computer Networks, vol. 38 pp. 393-422, Dec. 2002.

[51] I. F. Akyildiz, W. Su, Y. Sankarasubramaniam, and E. Cayici," A survey on Sensor Networks," IEEE Communications Magazine, pp. 102-114, Aig. 2002.

[52] C. Intanagonwiwat, R. Govindan, and D. Estrin, "Directed diffusion: a scalable and robust communication paradigm for sensor networks," Proceedings of the ACM Mobi-Com'00, Boston, MA, 2000, pp. 56-67.

[53] G.J. Pottie and W.J. Kaiser, "Wireless integrated network sensors," Communications of the ACM vol. 43 Issue 5, May 2000, pp. 51-58.

[54] S. Meguerdichian, F. Koushanfar, G. Qu, M. Potkonjak, "Exposure in wireless ad-hoc sensor networks," Proceedings of ACM MobiCom'01, Rome, Italy, 2001, pp. 139-150. 
[55] A. Elahi and A. Gschwender, "ZigBee Security," in Wireless Sensor and Control Network, Prentice Hall, October 29, 2009, pp. 288.

$[56$
M. Rouse,
"IR wireless
(infrared
wireless),"
Internet:

http://searchmobilecomputing.techtarget.com/definition/IR-wireless, Sep. 2005, [Dec. 2012].

[57] "ZigBee Wireless Standard - Technology - Digi International," Digi International website, Internet: http://www.digi.com/technology/rf-articles/wireless-zigbee, 2011 [ Dec. 5, 2012].

[58] S. Farahani, "ZigBee Basics" in Designing ZigBee Networks and Transceivers: The Complete Guide for Rf/Wireless Engineers, Elvsevier Ltd, 2008, pp. 15.

[59] M. A. Qureshi, "Teach yourself PIC Microcontrollers", Microtronics Pakistan, Pakistan Available: http://electronicspk.com/xcart/files/amer/teachyourselfpic.pdf, Nov.17, 2011 [Dec. 05, 2012]

[60] "USART", Microchip Internet: http://ww1.microchip.com/downloads/en/devicedoc/30292c.pdf, 2001 [Dec, 2012].

[61] "XBee ${ }^{\text {TM }}$ ZNet 2.5/XBee-PRO ${ }^{\mathrm{rm}}$ ZNet 2.5 OEM RF Modules" http://ftp1.digi.com/support/documentation/90000866 C.pdf, Feb. 2008 [Dec., 2012].

[62] "X-CTU Configuration and Test Utility Software user's Guide" Internet: http://www.scribd.com/doc/71254192/Config , [Dec., 2012].

[63] "Couple charged with elder abuse remains in custody" Internet: http://toronto.ctvnews.ca/couple-charged-with-elder-abuse-remains-in-custody-1.613582, Mar. 1, 2011 [Dec., 2012]. 
[64] M. Wardrop, "'Neglect' fears after elderly couple die in freezing home," Telegraph avaialble: http://www.telegraph.co.uk/topics/weather/6970522/Neglect-fears-after-elderly-couple-die-infreezing-home.html, Jan. 12, 2010 [2012].

[65] "Water heater's monoxide leak kills elderly couple," The China Post/Asia News Network, Tuesday, internet: http:/www.asiaone.com/News/Latest\%2BNews/Asia/Story/A1Story20111213315944.html, Dec 13, 2011, [Dec. 31, 2012]

[66] E. M. Tapia, "Activity Recognition in the Home Setting Using Simple and Ubiquitous Sensors," Dept. Media Arts and Sciences, Masschusetts Institute of Technology(MIT), Cambridge, Massachusetts, United States, 2003.

[67] "How does ZigBee compare with other wireless standards?" Software Technologies Group, Inc, Internet: http://www.stg.com/wireless/ZigBee comp.html, Oct. 25, 2011 [Dec.2012].

Serial Adapter Baud Rate" Available: http://www.digi.com/wiki/developer/index.php/Setting Serial Adapter Baud Rate, Sept. 2009 [Dec., 2012].

[69] V. Milan, "Introduction: World of microcontrollers", in PIC Microcontrollers, 1st ed., mikroElektronika, 2008, pp. 394.

[70] "Maxbotix Ultrasonic Rangefinder - LV-EZ1 - EZ-1" Available: http://www.maxbotix.com/documents/MB1010 Datasheet.pdf, Oct.28, 2011, [Dec. 05, 2012].

[71] S. Nathan, "Beginning Embedded Electronics - 1" Internet: http://www.sparkfun.com/tutorials/57, June 19, 2008, [Dec. 05, 2012]. 
[72] K. Manabe, T. Matsui, M. Yamaya, T. Sato-Nakagawa, N. Okamura, H. Ari, and H.Sasaki. "Sleeping patterns and mortality among elderly patients in geriatric hospitals". Gerontology, vol. 46: pp 318-322, 2000.

[73] T. Anneliis, "Remote Medical Decision Support System and Use Interface Usability" M.S. Thesis, Dept., Comp. and Sys., Carleton University, Ottawa, ON, Canada, 2010.

[74] I. Veledar, "Patient Bed Transfer Analysis using Pressure Sensor Arrays," M. S., Dept., Comp. and Sys., Carleton University, Ottawa, ON, Canada, 2009.

[75]

N. Foubert, "Posture Recognition and Postural Transition Detection Using Bed-Based Pressure Sensor Arrays," M. S., Dept., Comp. and Sys., Carleton University, Ottawa, ON, Canada, 2010.

[76] E. Hanna, "Sterescopic Imaging for Obstacle Detection Onboard Low-Flying Unmanned Aerial Vehicles," M. S., Dept., Comp. and Sys., Carleton University, Ottawa, ON, Canada, 2010.

[77] Gaddam, S.C. Mukhopadhyay, and G.S. Gupta, "Smart home for elderly care using optimized number of wireless sensors" 4th International Conference on Computers and Devices for Communication, Dec. 14-16, 2009, pp. 1-4

[78] S.S.Riaz Ahamed, "THE ROLE OF ZIGBEE TECHNOLOGY IN FUTURE DATA COMMUNICATION SYSTEM", proc. Journal of Theoretical and Applied Information Technology, vol. 5, 2009, pp 129-135.

[79] Werner-Allen, G., P. Swieskowski, and M. Welsh, "Motelab: A Wireless Sensor Network Testbed," IPSN 2005 Fourth International Symposium in Information Processing in Sensor Networks, 2005, pp. 483- 488.

[80] C.S. Raghavendra, K.M. Sivalingam, and T. Znati, "Wireless Sensor Networks," ERCOFTAC Series, Springer, 2004 
[81] S. Ohta, H. Nakamoto, Y. Shinagawa, and T. Tanikawa, "A health monitoring system for elderly people living alone", Journal of Telemedicine and Telecare, vol 8, 2002,PP. 151-156.

[82] G. LeBellego, N. Noury, G. Virone, M. Mousseau, and J Demongeot, "A model for the measurement of patient activity in a hospital suite", IEEE Transactions on Information Technology in Biomedicine, vol. 10, PP. $92-99$, Jan. 2006

[83] R. Faludi, Building Wireless Sensor Networks with ZigBee, XBee, Arduino, and Processing, Sebastopol, Ca, 1st ed., O'Reilly Media, Inc., 2010, pp. 322.

[84] A.R Kaushik, and B.G Celler, "Characterization of Passive Infrared Sensors for Monitoring Occupancy Pattern" in proc. IEEE 28th Annual International Conference of the Engineering in Medicine and Biology Society (MBS '06), New York City, USA, Aug 30-Sept 3, 2006 PP. 5257 - 5260.

[85] T.C.Manjunath, A. Kusagur , S. Sanjay, S. Sindushree, C. Ardil "Design, Development \& Implementation of a Temperature Sensor using Zigbee Concepts" proc. International Journal of Electrical and Computer Engineering (IECE), 2008, pp $736-748$.

[86] Tran, S.P.M. and T.A. Yang. "Evaluations of target tracking in wireless sensor networks", in Proc. of the 37th SIGCSE technical symposium on Computer science education SIGCSE '06, Vol. 38, Issue 1, Mar. 2006, pp. 97-101.

[87] X. H. B. Le, M. Di Mascolo, A. Gouin, and N. Noury, "Health Smart Home - Towards an assistant tool for automatic assessment of the dependence of elders", in proc. 29th IEEE-EMBS2007, Lyon, France, 2007, pp. 3806-09

[88] X. H. B. Le, M. Di Mascolo, A. Gouin, and N. Noury, "Health Smart Home for elders - A tool for automatic recognition of activities of daily living", in proc. 30th Annual international IEEE EMBS Conference Vancouver, Canada, 2008, PP. 3316-3319. 
[89] A. Gaddam, K.Kaur, S. C. Mukhopadhyay and G. Sen Gupta "Selection and Optimization of Wireless Sensors in a Smart Digital Home for the Elderly" proc IEEE SENSORS, Oct. 25-28, 2009, pp 1383-1386.

[90] E. M. Tapia, S. I. Stephen, and K. Larson, "Activity Recognition in the Home Using Simple and Ubiquitous Sensors" Lecture Notes in Computer Science, Massachusetts Institute of Technology 1 Cambridge Center, 4FL Cambridge, MA 02142 USA, Vol. 3001/2004, pp 158-175, 2004.

[91] T. S. Barger, D. E. Brown, and M. Alwan, "Health-status monitoring through analysis of behavioral patterns" Proc. IEEE Transactions on Systems, Man and Cybernetics, Part A: Systems and Humans, vol. 35, no. 1, pp. 22-27, Jan. 2005.

[92] B. Seung-Ho, P. Jae-Han, J. Koh, K.-W. Park, M.-H. Baeg; "Building a smart home environment for service robots based on RFID and sensor networks "proc. International Conference on Control, Automation and Systems, ICCAS '07," Oct. 17-20, 2007, pp 1078-1082.

[93] X. Ming; M. Longhua ; X. Feng; Y. Tengkai ; Q. Jixin ; and S. Meng; "Design and Implementation of a Wireless Sensor Network for Smart Homes" 2010 7th International Conference on Ubiquitous Intelligence \& Computing and 7th International Conference on Autonomic \& Trusted Computing (UIC/ATC), 26-29 Oct Dec. 2010, PP. $39-243$.

[94] F. G. Serrano Moya and J. L. Zamorano Flores "Wireless Sensor Network for Monitoring Temperature" $1^{\text {st }}$ International Congress on Instrumentation and Applied Sciences, pp. 1-7, Oct. 2010.

[95] Report on the Demographic Situation in Canada," Satisitics Canda, Internet: http://dsppsd.pwgsc.gc.ca/collection 2008/statcan/91-209-X/91-209-XIE2004000.pdf, July, 2008, [Dec. 05, 2012

[96] Public Health Agency of Canada. Available: http://www.phac-aspc.gc.ca, Oct. 25, 2011 [Dec. 05, 2012]. 
[97] S. Hussain and S. Dar, "Architecture for smart sensors system for tele-health," in IEEE International Workshop on Health Pervasive Systems, Available: http://cs.acadiau.ca/ shussain/wsn/publications/06-icps-hps-hussain.pdf, June 29, 2006, [Dec. 05, 2012].

[98] N. Noury, "AILISA: experimental platforms to evaluate remote care and assistive technologies in gerontology,"Proc. the 7Th International Workshop on Enterprise Networking and Computing in Healthcare Industry, June 23-25, 2005, pp. 67-72.

[99] K. JeongGil; L. Chenyang; M.B. Srivastava; J.A. Stankovic; A.Terzis; M. Welsh; "Wireless Sensor Networks for Healthcare" Proc. of the IEEE, Vol. 98, Issue:11, PP: 1947-1960, Oct. 2010.

[100] H. Hongwei, X. Youzhi, Y. Hairong, S. Mubeen, and Z. Hongke, "An Elderly Health Care System Using Wireless Sensor Networks at Home", Third International Conference on Sensor Technologies and Applications (SENSORCOMM '09), 2009, PP. 158 - 163.

[101] D. Niu, M. Yang, Y. Zhao, and Y. Zhang, "The Networking Technology within Smart Home System - ZigBee Technology," International Forum on Compter Science-Technology and Applications (IFCSTA '09), 2009, PP. $29-33$.

[102] S. Baoye, T. Guohui, L. Guodong, Z. Fengyu, and L. Dongxu, "ZigBee based wireless sensor networks for service robot intelligent space", 2011 International Conference on Information Science and Technology (ICIST), 2011, PP. $834-838$.

[103] L. Shizhuang, L. Jingyu, F. Yanjun, "ZigBee Based Wireless Sensor Networks and Its Applications in Industrial", 2007 IEEE International Conference on Automation and Logistics, 2007, PP. 1979 1983. 
[104] H. Dae-Man and L. Jae-Hyun, "Design and implementation of smart home energy management systems based on zigbee", IEEE Transactions on Consumer Electronics, Vol. 56, Issue: 3, pp. 1417 1425, Oct. 2010.

[105] W. S. Jang, W.M. Healy, and M.J. Skibniewski, "Wirelesless sensor networks as part of a webbased building environmental monitoring system, " Automation in construction vol. 17, pp. 729-736, Feb. 2008.

[106] N. K. Suryadevara and S.C. Mukhopadhyay, "Wireless sensor network based home monitoring system for wellness determination of elderly," IEEE sensor journal, vol. 12, No. 6, pp. 1965-1972, Jan. 2012.

[107] W. M. Healy. "Lessons learned in wireless monitoring." ASHRAE journal, vol. 47, pp.54-60, Oct. 2005

[108] A.C. Wendel and S. E. Freitag, "Wireless system for detecting and stopping water leaks," U.S. Patent 5655 561, Aug. 12, 1997.

[109] Z. Rasin and M.R. Abdullah, "Water quality monitoring system using ZigBee based wireless sensor netowrk" International Journal of Engineering and Technology IJET, vol. 9, No: 10, pp. 24-28

[110] V. C. Gungor andd G. P. Hancke, "Industrial wireless sensor networks: challenges, design priciples, and technical approaches, "IEEE Transactions on Industrial Electronics, vol. 56, No. 10, pp. 4258-4265, Oct. 2009.

[111] A. Gaddam, S.C. Mukhopadhyay, G. S. Gupta, and H. Guesgen, "Wireless Sensors Networks Based Monitoring Review, Challenges and Implenetation," $3^{\text {rd }}$ International Conference on Sensing Technology, Tainan, Taiwan, Nov. 30 - Dec. 3, 2008, pp. 533-538. 
[112] B. Dong and B. Adrews, " Sensor-Based Occupancy Behavioral Pattern Recognition for Energy and Comfort management in Intelligent Building," Eleventh International IBPSA conference, Glasgow, Scotland, July 27-30, 2009, pp. 1444-1451.

[113] T. Arampatzis, J. Lygeros, and S. Manesis, " A survey of Applications of Wireless Sensors and Wireless Sensor Networks," proc. 2005 Control and Automation Mediterranean Conf., Jun. 27-29, 2005, pp. 719-724.

[114] B.G. Celler et al. "Remote monitoring of health status of the elderly at home. A multidisciplinary project on aging at the Univeristy of New South Wales," International Journal of Bio-Medical computing, vol. 40 pp. 147-155, 1995.

[115] L. Farinaccio and R. Zmeureanu, "Using a Pattern recognition approach to disaggregate the total electricity consumption in a house into the major end-uses," Energy and Building, vol. 30 pp. 245259, Feb. 1999.

[116] R. V. Sakhare, and B.T. Deshmukh, “Electric Power Management Using ZigBee Wireless Sesor Network," International Journal ofAdvances in Engineering and Technology IJAET, vol. 4, Issue 1, pp. 492-500, July, 2012.

[117] D.-M. Han and J.-H. Lim, " Smart home Energy Management System using IEEE 802.15.4 and ZigBee,"

[118] L. Ruiz-Garcia et al." A Review of Wireless Sensor Technologies and Applications in Agriculture and Food : State of the Art and Current Trends,"Journal of Sensors, vol. 9, pp. 4728-4750, 2009.

[119] M. Kintner-Meyer and M. R. Brambley, "Pros \& Cons of Wireless," ASHRAE journal, pp.54-59, Nov. 2002. 
[120] B. Krose, T. V. Kasteren, C. Gibson, and T. V. Dool, "CARE: Context Awareness in Residences for Elderly," The $6^{\text {th }}$ International Conference of the Society for Gerontechnology, Pisa2008, pp. 101-105.

[121] J. Torresen, E. Renton and A. R. Jensenius, "Wireless Sensor Data Collection based on ZigBee Communication," proc. 2010 Conference on Interfaces for Musical Expression (NIME 2010), Sydney, Austrilia, 2010, pp. 368-371.

[122] A. Milenkovic, C. Otto, and E. Jovanov, "Wireless sensor networks for personal health monitoring: Issues and an implementation,"Computer Communications, vol. 29, pp. 2521-2533,Feb. 2006.

[123] M. Chan, E. Campo, D. Esteve, and J.-Y. Fourniols, "Smart homes - Current features and future perspectives," Mauritas, vol. 64, pp. 90-97, July, 2009.

[124] W.-T. Sung and M.-H. Tsai, "Multi-Sensor Wireless Signal Aggregation for Environmental monitoring System System via Mutli-bit Data Fusion," An International Journal of Applied Mathematics and Information Sciences, vol. 5, pp. 589-603, 2011.

[125] R. Zhang, Z. Jia, and D. Yuan, "Analysis of lifetime of Large Wireless Sensor Networks Based on Mutliple Levels," International Journal of Communications, Network and System Sciences, vol. 2, pp. 105-206, 2008.

[126] R. Suzuki, M. Ogawa, S. Otake, T. Izutsu, Y. Tobimatsu, S.-I. Izumi and T. Iwaya, "Analysis of Activities of Daily Living in Elderly People Living Alone: Single-Subject Feasibility Study,"Telemedicine journal and e-Health, vol. 10, No. 2, pp.260-276,2004.

[127] H. S. Mathews, L. Soibelman, M. Berges, and E. Goldman, "Automatically Disaggregating the Total Electrical Load in Residential Buildings: a Profile of the Required Solution," Intelligent Computing in Engineering - ICEO8, pp.381-389, 2008. 
[128] K. F. Tsang, K.L. Lam, and H.Y. Tung, "Energy management Using ZigBee," 2009 Third International Conference on Next Generation Mobile Applications, Service and Technologies, pp. 412 415, Sept. 15-18 2009.

[129] A. O. Fapojuwo, C. K. Tse, and F. C. M. Lau, "Energy Consumption in Wireless Sensor Networks under Varying Sensor Node Traffic," Wireless Communications and Networking Conference (WCNC), Hong Kong, China, pp. 106, April 18-21, 2010.

[130] G. Wittenburg, N. Dziengel, C. Wartenburger, and J. Schiller,"A System for Distributed Event Detection in Wireless Sensor Networks," the 9th ACM/IEEE International Conference on Information Processing in Sensor Networks, Stockholm, Sweden, pp. 94-104, April 12 - 15, 2010.

[131] A. Wood, J. Stankovic, G. Virone, L. Selavo, Z. He, Q. Cao, T. Doan, Y. Wu, L. Fang, and R. Stoleru, "Context-Aware Wireless Sensor Networks for Assisted Living and Residential Monitoring" IEEE Network, vol.22, pp. 26-33, Aug. 2008.

[132] S.-W. Luan, J.-H. Teng, S.-Y. Chan, and L.-C. Hwang "Development of a Smart Power Meter for AMI Based on ZigBee Communication" International Conference on Power Electronics and Drive Systems (PEDS) pp. 661-665, Nov. 2-5, 2009.

[133] "Towards 100\% Reliability in Wireless Monitoring Networks" the 3rd ACM international workshop on Performance evaluation of wireless ad hoc, sensor and ubiquitous networks, New York, NY, USA, 2006, pp. 132-135.

[134] R. Srinivasan, C. Chen and D. J. Cook, "Activity Recognition using Actigraph Sensor," available: http://eecs.wsu.edu/ cook/pubs/kdd10p3.pdf, July 25, 2010 [Dec. 05, 2012].

[135] K. V. Laerhoven and H.-W. Gellersen, "Multi Sensor Context Awareness," avaialable: http://ubimon.doc.ic.ac.uk/bsn/public/Kristof van Laerhoven - abstract.pdf, [Dec. 05, 2012]. 
[136] N. Javaid, A. Sharif, A. Mahmood, S. Ahmed, U. Qasim, and Z. A. Khan, "Monitoring and Controlling Power using ZigBee Communications," 2012 7th IEEE International Conference on BWCCA, Victoria, Canada, http://arxiv.org/abs/1208.2331] Aug. 11, 2012, [Dec. 05, 2012].

[137] G. Pekhteryev, and Z. Sahinoglu, "Multi-stage Real TimeHealth Monitoring via ZigBee in Smart Homes," 21st International Conference on Advanced Information Networking and Applications Workshops-Cover, vol.2, May 21-23, 2007, pp. 782 - 786.

[138] Z. Hu and B. Li, "Fundamental Performance Limits of Wireless Sensor Networks," available: http://iqua.ece.toronto.edu/ bli/papers/limits.pdf, 2004,[Dec. 05, 2012].

[139] S. Jung, A. Chang and M. Gerla, "Comparisons of ZigBee Personal Area Network (PAN) Interconnection Methods," 4th International Symposium on Wireless Communication Systems (ISWCS), pp. $337-341,2007$.

[140] O. Landsiedel, K. Wehrle, and S. Gotz, "Accurate Prediction of Power Consumption in Sensor Network," The Second IEEE Workshop on Embedded Networked Sensors, pp. 37 - 44, 2005.

[141] E. M. Tapia, S. S. Intille, and K. Larson, "Activity Recognition in the Home Using Simple and Ubiquitous Sensors," http://web.media.mit.edu/ intille/papers-files/TapialntilleLarson04.pdf, 2004, [Dec.05, 2012].

[142] I. Khemapech, I. Duncan and A. Miller, "A survey of Wireless Sensor Networks Technology" http://www.cs.st-andrews.ac.uk/ ik/index.files/IKPGNET2005.pdf, 2005, [Dec. 05, 2012].

[143] C. Pham and P. Olivier, "Slice\&Dice: Recognition Food Preparation Activities Using Embedded Accelerometers," Proc. of the European Conference on Ambient Intelligence, Vol. 5859, (2009), pp. 34-43. 
[144] E. O. Heierman, G. M. Youngblood, and D. J. Cook "Mining Temporal Sequences to Discover Interesting Patterns," August 22-25, 2004.

[145] S. Vurgun, M. Philipose, and M. Pavel "A Statistical Reasoning System for Medication Prompting," proc. the 9th international conference on Ubiquitous computing, 2007, pp. 1-18.

[146] M. D. FRANCESCO, S. K. DAS, and G. ANASTASI, "Data Collection in Wireless Sensor Networks with Mobile Elements: A Survey," ACM Transactions on Sensor Networks, Vol. 8, No. 1, Issue 1, Aug. 2011.

[147] A. Dunkels, F. Osterlind, and Zhitao He, "An Adaptive Communication Architecture for Wireless Sensor Networks,"proc. the 5th international conference on Embedded networked sensor systems, Sydney, Austrilia, ACM New York, NY, USA, pp. 335-349, Nov. 6-9, 2007.

[148] O. Chipara1, C. Lu, T. C. Bailey, and G.-C. Roman "Reliable Clinical Monitoring using Wireless Sensor Networks: Experiences in a Step-down Hospital Unit, " proc. the 8th ACM Conference on Embedded Networked Sensor Systems, Zurich, Switzerland, Nov. 3-5, 2010,pp. 155-168.

[149] P. P. Jayaraman, A. Zaslavsky, and J. Delsing, "Sensor Data Collection Using Heterogeneous Mobile Devices," IEEE International Conference on Pervasive Services, July 15-20, 2007, pp. 161 164.

[150] A. Wood, J. Stankovic, G. Virone, L. Selavo, Z. He, Q. Cao, T. Doan, Y. Wu, L. Fang, and R. Stoleru, "Context-aware wireless sensor networks for assisted living and residential monitoring", IEEE Network, vol. 22, no. 4, pp. 26-33, Aug. 2008.

[151] L. Insup, J. Y-T. Leung, H. S. Sang, "Introduction" in Handbook of real-time and embedded systems, New York: Tayler and Francis Group, 2008, pp. 20. 
[152] B. Zhou, A. Marshall, W. Zhou and T.-H. Lee, "Novel Wire- less Mesh Networking Architectures for Future Smart Homes," Future Generation Communication and Net-working (FGCN 2007), Vol. 2, December 2007, pp. 43-48.

[153] "Wireless Sensor Network (WSN)" Internet: http://www.techopedia.com/definition/25651/wireless-sensor-network-wsn, Oct. 25, 2011 [Dec. 05, 2012]. 


\section{APPENDIX}

\section{Appendix A: Wireless Technologies}

The advantages and disadvantages of the potential wireless technologies are following:

\section{Advantages of Wi-Fi are summarized below:}

- Good for moving large amounts of data $(1 \mathrm{Mb}+)$;

- Employs unlicensed radio spectrum and does not need regulatory approval for individual launch;

- Has a set of global standards, which means it can operate in different countries around the world at all times; and

- Supports roaming: it allows mobile users with devices such as laptop computers to be able to move from one access point to another.

\section{Disadvantages of Wi-Fi are summarized below:}

- Consumes larger amounts of power compared to some other standards such as XBee, Wireless USB, or Bluetooth. Wi-Fi requires approximately 100mA to handle 802.11 stacks, making the battery life and heat a major concern to some users; and

- Wi-Fi networks have limited range depending on the physics of the radio wave propagation

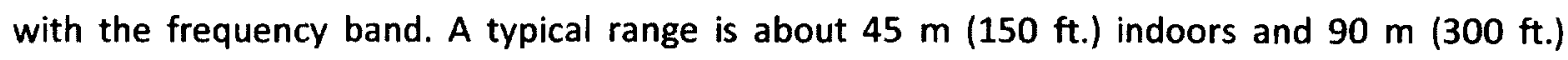
outdoors.

The advantages of Bluetooth are summarized as follows: 
- Can be used in both fixed devices, such as telephones, computers, printers and other devices, in mobile devices, such as cell phones, laptops, and other devices, and also for creating personal area network (PANs);

- Is easy to use;

- Certified interoperability: it has the capability for diverse systems and organizations to work together;

- The range is between 50-100 meters, suitable for Bluetooth applications; and

- Also supports audio links.

The disadvantages of Bluetooth are the following:

- Uses the same frequency as the WaveLAN ${ }^{18}$ standard, which may cause interference; and

- Is omni-directional; it encounters problems in determining the intended recipient device.

\section{Ultra wide band (UWB)}

These applications are summarized below. Ultra wide band applications can be divided into two forms: communications and radars.

(1) Ultra wide band can be used for the following applications in communications:

- High-speed, multi-user wireless networks;

- Wireless personal area networks (PAN)/local area networks (LAN);

${ }^{18}$ WaveLAN is a trade name that describes two completely different families of wireless network solutions:

- Pre-IEEE 802.11 WaveLAN, also called Classic WaveLAN

- IEEE 802.11-compliant WaveLAN, also known as WaveLAN IEEE or ORINOCO

Source: en.wikipedia.org/wiki/WaveLAN 
- Indoor video/data/voice distribution; and

- Military applications.

(2) Ultra wide band can be used for the following applications in radars:

- Through-wall imaging and motion sensing radar;

- Security systems for alarming and tracking movement;

- Underground imaging;

- Vehicular radar: collision avoidance/detection, road conditions sensing; and

- Military applications (intrusion detection radars, proximity fuses, unmanned ground and aerial vehicles).

Advantages of the Ultra wide band (UWB) are summarized below:

- One of the most significant of these advantages is its capability to relatively easily share spectrum space with other devices. Since their frequencies are so adaptive, the devices can easily change to locate an open range in which to transmit;

- UWB communications transmit in a way that does not interfere largely with other more traditional narrowband and continuous carrier wave uses in the same frequency band;

- High capacity for frequency band;

- Multipath robustness;

- Position location capability;

- Low transmission power;

- Low implementation cost; and

- Multi-access capability. 
Disadvantages of the Ultra wide band (UWB) are summarized below:

- Power limitation; and

- Synchronization.

Advantages of the Infrared (IR) are summarized below:

- Consumes low power: ideal for devices such as laptops, telephones, personal digital assistants'

- Inexpensive: costs around \$2-5 for the entire coding/decoding circuitry;

- IR circuitry is very simple. No special or proprietary hardware is necessary and it can be incorporated into the integrated circuit of a product;

- XBee has higher security compared to other wireless technologies because of the directionality of the beam, which helps ensure that data is not leaked or spilled to nearby devices as it is transmitted;

- It is portable;

- No restrictions by international regulations for devices to be functional, and can be used by anyone especially by international travelers, no matter where they may are with no restrictions; and

- No interference from signals from other devices.

\section{Disadvantages of the Infrared (IR) are summarized below:}

- The technology is not like radio-frequency (RF) wireless links in that IR wireless cannot penetrate through walls. IR wireless communications or control is generally not possible between different rooms in a house or between different houses in a neighbourhood except when they are facing 
windows. Any blockage by common objects, such as people, animal, walls, plants, can obstruct the transmission of data;

- It must be almost directly in the line of sight for devices to transmit and receive data;

- Performs well for short range; however, the range drops off with longer distance;

- Transmission can be affected by elements such as light, weather, sunlight, rain, fog, dust, or pollution; and

- Data rate transmission is slower than typical wired transmission.

\section{Radio Frequency Identification (RFID)}

Advantages of the Radio frequency identification (RIFD) are summarized below:

- Non-line-of-sight operation;

- Higher inventory rates; and

- Rewritable product IDs.

Table 7.1 provides a summary of the potential wireless technologies, including Wi-Fi, Bluetooth, Ultra wide band (UWB), Wireless Universal Serial Bus (USB), Infrared (IR) wireless, and ZigBee. 


\begin{tabular}{|c|c|c|c|c|c|c|}
\hline & ZigBee & $\begin{array}{l}802.11 \\
(W i-F i)\end{array}$ & Bluetooth & $\begin{array}{l}\text { UWB (UItra } \\
\text { Wide Band) }\end{array}$ & Wireless USB & IR Wireless \\
\hline Data Rate & $\begin{array}{c}20,40, \text { and } 250 \\
\mathrm{Kbits} / \mathrm{s}\end{array}$ & $11 \& 54 \mathrm{mbits} / \mathrm{sec}$ & 1 Mbits/s & 100-500 Mbits/s & $62.5 \mathrm{Kbits} / \mathrm{s}$ & $\begin{array}{c}20-40 \mathrm{Kbits} / \mathrm{s} \\
115 \mathrm{Kbits} / \mathrm{s} \\
4 \& 16 \mathrm{Mbits} / \mathrm{s}\end{array}$ \\
\hline Range & $10-100$ meters & 50 - 100 meters & 10 meters & $<10$ meters & 10 meters & $\begin{array}{l}<10 \text { meters (line } \\
\text { of sight) }\end{array}$ \\
\hline $\begin{array}{l}\text { Networking } \\
\text { Topology }\end{array}$ & $\begin{array}{c}\text { Ad-hoc, peer to } \\
\text { peer, star, or } \\
\text { mesh }\end{array}$ & Point to hub & $\begin{array}{c}\text { Ad-hoc, very } \\
\text { small networks }\end{array}$ & Point to point & Point to point & Point to point \\
\hline $\begin{array}{l}\text { Operating } \\
\text { Frequency }\end{array}$ & $\begin{array}{c}868 \mathrm{MHz} \text { (Europe) } \\
900-928 \mathrm{MHz} \\
\text { (NA), } 2.4 \mathrm{GHz} \\
\text { (worldwide) }\end{array}$ & 2.4 and $5 \mathrm{GHz}$ & $2.4 \mathrm{GHz}$ & $3.1-10.6 \mathrm{GHz}$ & $2.4 \mathrm{GHz}$ & $800-900 \mathrm{~nm}$ \\
\hline $\begin{array}{l}\text { Complexity } \\
\text { (Device and } \\
\text { application } \\
\text { impact) }\end{array}$ & Low & High & High & Medium & Low & Low \\
\hline $\begin{array}{l}\text { Power } \\
\text { Consumption } \\
\text { (Battery option } \\
\text { and life) }\end{array}$ & $\begin{array}{c}\text { Very low (low } \\
\text { power is a design } \\
\text { goal) }\end{array}$ & High & Medium & Low & Low & Low \\
\hline Security & $\begin{array}{c}128 \text { AES plus } \\
\text { application layer } \\
\text { security }\end{array}$ & & $\begin{array}{l}64 \text { and } 128 \text { bit } \\
\text { encyption }\end{array}$ & & & \\
\hline $\begin{array}{l}\text { Other } \\
\text { Information }\end{array}$ & $\begin{array}{l}\text { Devices can join } \\
\text { an existing } \\
\text { network in under } \\
\text { 30ms }\end{array}$ & $\begin{array}{l}\text { Device connection } \\
\text { requires 3-5 } \\
\text { seconds }\end{array}$ & $\begin{array}{l}\text { Device connection } \\
\text { requires up to } 10 \\
\text { seconds }\end{array}$ & & & \\
\hline $\begin{array}{l}\text { Typical } \\
\text { Applications }\end{array}$ & $\begin{array}{c}\text { Industrial control } \\
\text { and monitoring, } \\
\text { sensor networks, } \\
\text { building } \\
\text { automation, } \\
\text { home control and } \\
\text { automation, toys, } \\
\text { games }\end{array}$ & $\begin{array}{l}\text { Wireless LAN } \\
\text { connectivity, } \\
\text { broadband } \\
\text { Internet access }\end{array}$ & $\begin{array}{c}\text { Wireless } \\
\text { connectivity } \\
\text { between devices } \\
\text { such as phones, } \\
\text { PDA, laptops, } \\
\text { headsets }\end{array}$ & $\begin{array}{l}\text { Streaming video, } \\
\text { home } \\
\text { entertainment } \\
\text { applications }\end{array}$ & $\begin{array}{l}\text { PC peripheral } \\
\text { connections }\end{array}$ & $\begin{array}{l}\text { Remote controls, } \\
\text { PC, PDA, phone, } \\
\text { laptop links }\end{array}$ \\
\hline
\end{tabular}

Table 7.1: Comparison of ZigBee with other wireless technologies [67] 


\section{Appendix B: XBee Module}

Communication is easy to establish with the XBee module because the interface used for sending and receiving data is UART (TTL). All it needs is to connect the UART communication pins of the XBee module directly to the microcontroller's UART. The XBee module is a half-duplex (HDX) module that provides information in both directions, but only one direction at a time (not simultaneously). Usually, when an XBee module begins receiving a signal, it must wait for the transmitter to stop transmitting before replying to the transmitter. Table 7.2 illustrates the basic feature of XBee modules.

\begin{tabular}{|c|c|}
\hline Wireless Type & ZigBee (XBee) \\
\hline Wireless Size & $0.962^{\prime \prime} \times 1.2972^{\prime \prime}(2.438 \mathrm{~cm} \times 3.294 \mathrm{~cm})$ \\
\hline Transmission & $250 \mathrm{kbps}$ \\
\hline Frequency Band & $2.4 \mathrm{GHz}$ \\
\hline Communication distance & $\begin{array}{l}\text { Indoor Urban: up to } 100^{\circ}(30 \mathrm{~m}) \\
\text { Out door line-of-sight: up to } 300^{\prime} \\
(100 \mathrm{~m})\end{array}$ \\
\hline Network Topology & $\begin{array}{l}\text { - Point to point } \\
\text { - Peer to peer } \\
\text { - Point to multipoint }\end{array}$ \\
\hline Supply Voltage & $2.8-3.3 \mathrm{~V}$ \\
\hline Manufacturer & Digi Intemational \\
\hline
\end{tabular}

Table 7.2: Features of XBee

\section{XBee and XBee-PRO RF Modules}

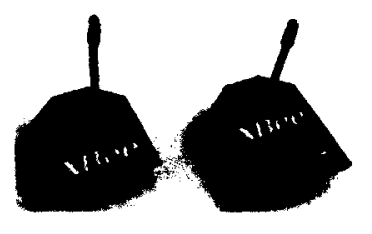

The component is available in both a low-power XBee and XBee-PRO RF version. The XBee-PRO RF has an extra amplifier for higher ranges; nevertheless, the power required to run The $\mathrm{XBee-PRO} R F$ is also a lot higher. Choosing the best or most suitable modules depends on the application (e.g., the purpose, or situation, of the research) and the users' request. Both the XBee and XBee-PRO RF modules are bidirectional bands and are pin-for-pin compatible with each other. The one to the right is of XBee Series 2 and the one to the left is XBee Pro Series 2; both modules are equipped with whip antennae. Table 7.3 is the 
comparison of the specifications of XBee and XBee Pro, including brief descriptions of their ranges, transmitter and receiver power, and sequence channels.

\begin{tabular}{|c|c|c|}
\hline Item & XBee & XBee Pro \\
\hline Range, Indoor Urban & 100 Feet (30 meters) & $\begin{array}{c}300 \text { feet ( } 90 \text { meters) } 200 \\
\text { feet ( } 60 \text { meters) for } \\
\text { International variant }\end{array}$ \\
\hline $\begin{array}{c}\text { Range, Outdoor line-of- } \\
\text { sight }\end{array}$ & 300 feet ( 90 meters) & $\begin{array}{c}1 \text { mile (1600 meters), } 2500 \\
\text { feet }(750 \text { meters }) \text { for } \\
\text { International variant }\end{array}$ \\
\hline Transmit Power & $1 \mathrm{~mW}(0 \mathrm{dBm})$ & $\begin{array}{c}63 \mathrm{~mW}(18 \mathrm{dBm}), 10 \mathrm{~mW} \\
(10 \mathrm{dBm}) \text { for Intermational } \\
\text { variant }\end{array}$ \\
\hline Receiver Sensitivity & $-92 \mathrm{dBm}$ & $-100 \mathrm{dBm}$ \\
\hline TX Peak Current & $45 \mathrm{~mA}(@ 3.3 \mathrm{~V})$ & $\begin{array}{l}250 \mathrm{~mA} \text { ( } 150 \mathrm{~mA} \text { for } \\
\text { intemational variant) } \\
340 \mathrm{~mA} \text { ( } 180 \mathrm{~mA} \text { for } \\
\text { international variant }\end{array}$ \\
\hline RX Current & $50 \mathrm{~mA}(@ 3.3 \mathrm{~V})$ & $55 \mathrm{~mA}(@ 3.3 \mathrm{~V})$ \\
\hline Power-down Current & $<10 \mu \mathrm{A}$ & $<10 \mu \mathrm{A}$ \\
\hline RF Data Rate & & $250,000 \mathrm{bps}$ \\
\hline Sequence Channels & 16 & 12 \\
\hline
\end{tabular}

Table 7.3: Different between XBee and XBee Pro [61]

\section{Types of Antennae}

There are the following different types of antenna choices available for the XBee modules that allow for connection to a dipole or other external antennae.

(1) Wire

(2) Chip

(3) UFL

(4) PCB 
(5) RP-SMA

The applications of XBee with these types of antennae depend on the users' application, for example:

\section{(1) The integrated whip or wire antenna and the chip antenna}

These kinds of antennae work well with any application but they are especially useful in embedded applications. They have no problem transmitting or receiving signals through plastic cases or housings; they can, therefore, be completely enclosed.

(i) Integrated whip or wire antenna uses Omni-directional radiation. This means that the

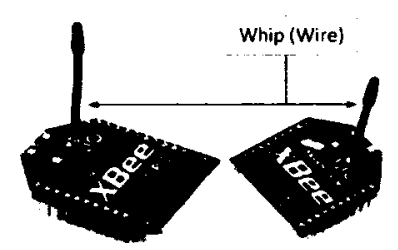
antenna is capable of transmitting or receiving signals in all directions, especially when the whip or wire is straight and perpendicular to the module as shown to the right.

(ii) Chip antennae have a triangle-shaped radiation pattern that allows the signal to attenuate

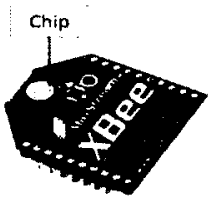
in many directions. The chip antenna is suitable for a device where mechanical stress to the wire antenna might break it or the module needs to be put in a very small space, such as anything wearable. The figure to the right shows the type of this chip antenna.

(2) The U.FL connector is used in conjunction with an adapter cable that can allow connection to a

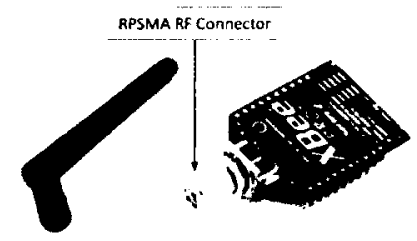
dipole or gain antenna as shown at Figure 7.1. The antenna is suitable for an XBee module that is embedded in metal or a solution that is more desirable mechanically. However, the antenna is to be left outside if the XBee module is going to be left on the inside of a metal box to avoid 
transmitting or receiving signals that should not be attenuated by the enclosure. In addition, it is sometimes beneficial to orient an external antenna differently than the XBee itself or to use a special-purpose antenna with a precise radiation pattern, such as a high-gain antenna that passes signals in a single direction over a broader distance. The U.FL connector is small, fairly fragile, and most of time used with a short connecting cable that carries the signal from a remotely mounted antenna.

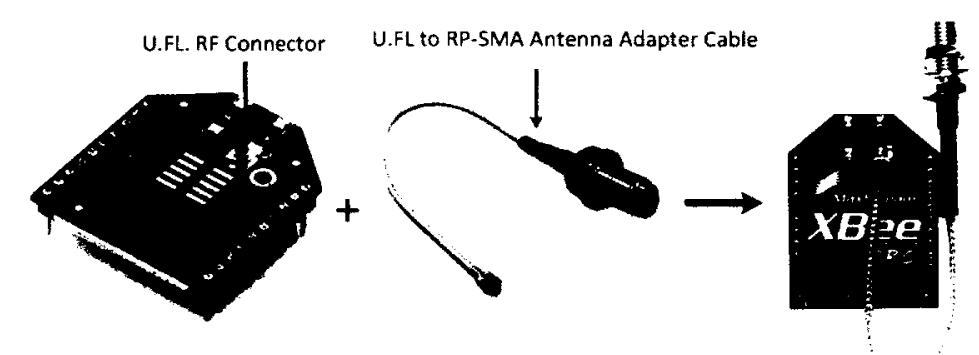

Figure 7.1: XBee module with type U.FL connector

(3) PCB antenna, which was established with the XBee-PRO $\$ 2 B$, is printed directly on the circuit board of the XBee. It is composed of a series of conducting traces set out in a fractal pattern. The PCB antenna offers many of the same benefits as the chip antenna and has a much lower manufacturing cost.

(4) RPSMA connector is just a different type of socket from the U.FL connector. It's known for its typically inconvenient size and for taking up a lot of space. It is large and unwieldy, but it can be used with an external antenna mounted directly to the XBee without a connecting cable. For most studies, it is still better off with the simple wire antenna that is smaller, cheaper, and generally just as good. That is why we used wire antennae for our study.

Although the $2.4 \mathrm{GHz}$ XBee module has a maximum over-the-air date rate of 250000 bps due to the overhead of the protocol, the actual theoretical maximum data rate is approximately half of that at 
15200 bps. The possible values are shown in Table 7.4. The Baud rate is managed by the BD setting, with standard Baud rates defined as the codes 0 to 7 [68]. 3(9600 bps) is set by factory default. The XBee module has low baud rates of between 1,200 and 115,200 bps.

\begin{tabular}{|c|c|}
\hline BD & Baud Rates \\
\hline 0 & 1200 bps \\
\hline 1 & 2400 bps \\
\hline 2 & 4800 bps \\
\hline 3 & 9600 bps \\
\hline 4 & 19200 bps \\
\hline 5 & 38400 bps \\
\hline 6 & 57600 bps \\
\hline 7 & 115200 bps \\
\hline
\end{tabular}

Table 7.4: Baud rates and parameter $(\mathrm{BD})$ defined as the codes 0 to 7 .

\section{Digi Development Board}

The Digi development board was also considered for the research. The board was included with a starter kit that we bought from Digi International for our research; however, for reasons of cost, size, and flexibility, we decided not to use it. The two types of these boards, USB and RS 232 Digi Development Boards, are shown at Figure 7.2. The boards are composed of a power supply source, USB or RS 232 connector, switch button, reset button, and lights. Although the boards are suitable for our base station, we decided to use the XBee adapter from SparkFun for our base station and Adafruit XBee adapter for our remote stations instead.
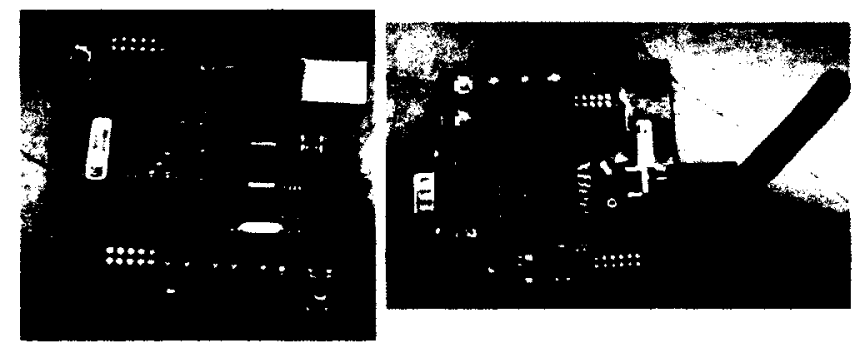

Figure 7.2: Digi International Development Boards for XBee 


\begin{tabular}{|c|c|c|c|}
\hline Component & Component limage & Manufacturer & $\begin{array}{l}\text { Cost (us } \\
\text { Dollar) }\end{array}$ \\
\hline $\begin{array}{l}\text { Zigbee/ } \\
\text { 802.13.4 } \\
\text { Modules RS- } \\
232 \text { XBene-PRO } \\
\text { pro onal } \\
\text { interface } \\
\text { board }\end{array}$ & 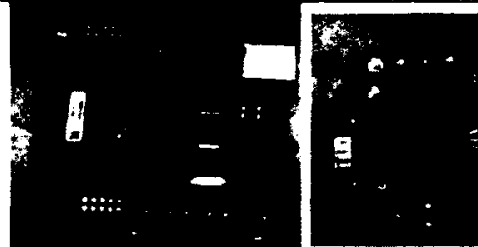 & $\begin{array}{c}\text { Digi } \\
\text { International }\end{array}$ & 79.90 \\
\hline $\begin{array}{l}\text { USB Mini-B } \\
\text { Cable- } 6 \text { Foot }\end{array}$ & & $\begin{array}{c}\text { Sparkfun } \\
\text { Electronics }\end{array}$ & 4.95 \\
\hline \multicolumn{3}{|c|}{ Total } & 84.85 \\
\hline
\end{tabular}

Table 7.5: USB and RS 232 XBee Development Board 


\section{Appendix C: Microcontroller}

The main purposes of the microcontroller in our research are as follows:

- Obtain raw samples of sensor inputs either in analogue or digital form;

- Convert these samples to digital binary;

- Convert these binary samples to binary-coded decimal (BCD); and

- Get ASCII code and compile information to be displayed at X - CTU Terminal.

\section{PIC 16 F876}

The brand of microcontroller that we used in our research is a series of microcontrollers called Peripheral Interface Controller (PIC) from Microchip manufacturer. Microcontroller PIC comes in different varieties, some with a basic low memory type, going all the way through analog-todigital converters (ADC) and to the even pulse-width modulation (PWM) built in.

The type of Microcontroller PIC that we used to capture the sensor's collected data is PIC 16F876, which is a microcontroller with 28 pins. It has an 8-Bit processor with a high-performance FLASH microcontroller, which means it can be erased and reprogrammed without having to use a UV light source. This allows the same device to be used for prototype development as well as production. The chip has $8 k$ of code space and $22 \mathrm{l} / 0$ lines (5 of which are a 10 bit analogy to the digital converter capable), 368 bytes of RAM, and 256 bytes of EEPROM. This microcontroller can run up to $20 \mathrm{MHz}$ with external crystal, and a package can be programmed in a circuit. It has a universal asynchronous serial transport (UART) for asynchronous serial communication, and a microchip family that is the synchronous serial port (MSSP) for Serial Peripheral Interface (SPIM) and Inter-Integrated Circuit $\left(I^{2} \mathrm{C}^{\mathrm{TM}}\right)$. 
The synchronous serial port can be configured as either 3 -wire $\mathrm{SPI}^{\mathrm{TM}}$ or the 2-wire $\mathrm{I}^{2} \mathrm{C}^{\mathrm{TM}}$ bus. Table 7.6 and Figure 7.3 illustrate the key features of this microcontroller.

\begin{tabular}{|c|c|}
\hline Parameter Name & Value \\
\hline Program Memory Type & Flash \\
\hline Program Memory (KB) & 14 \\
\hline CPU Speed (MIPS) & 5 \\
\hline RAM Bytes & 368 \\
\hline Data EEPROM (bytes) & 256 bytes \\
\hline Digital Communication Peripherals & 1-A/E/USART, 1-MSSP(SPI/12C) \\
\hline Capture/Compare/PWM Peripherals & 2 CCP \\
\hline Timers & $2 \times 8$-bit, $1 \times 16$-bit \\
\hline ADC & 5 channels, 10-bit \\
\hline Temperature Range & -40 to 85 \\
\hline Operating Voltage Range (V) & 2 to 5.5 V \\
\hline Pin Count & 28 pin \\
\hline Manufacturer & Microchip \\
\hline
\end{tabular}

Table 7.6: Characteristics of microcontroller PIC 16 F876

Before we can begin discussing the other components, we would like to describe asynchronous serial communication.

- RA4/TOCK1, pin 6 can be used as input or output (RA4), or can be used for a clock (TOCK1) input that operates an internal timer. It operates in isolation to the main clock. The same was also true in this thesis; once the pin was selected for one function, it was not available for any other function. For example, if the pin is used at input or output (RA4) then it is unavailable for the interruption monitoring (TOCK1).

- RBO/INT, pin 21 can be used for an input or output (RBO), or it can be used for interruption (INT) monitoring. For example, if the pin goes high, it can cause the program to restart, stop, or any other single function desired. Once the pin is selected for one function, it is not available for any other function. In other words, if the pin is used at input or output (RBO) then the pin is unavailable for the interruption monitoring (INT). 


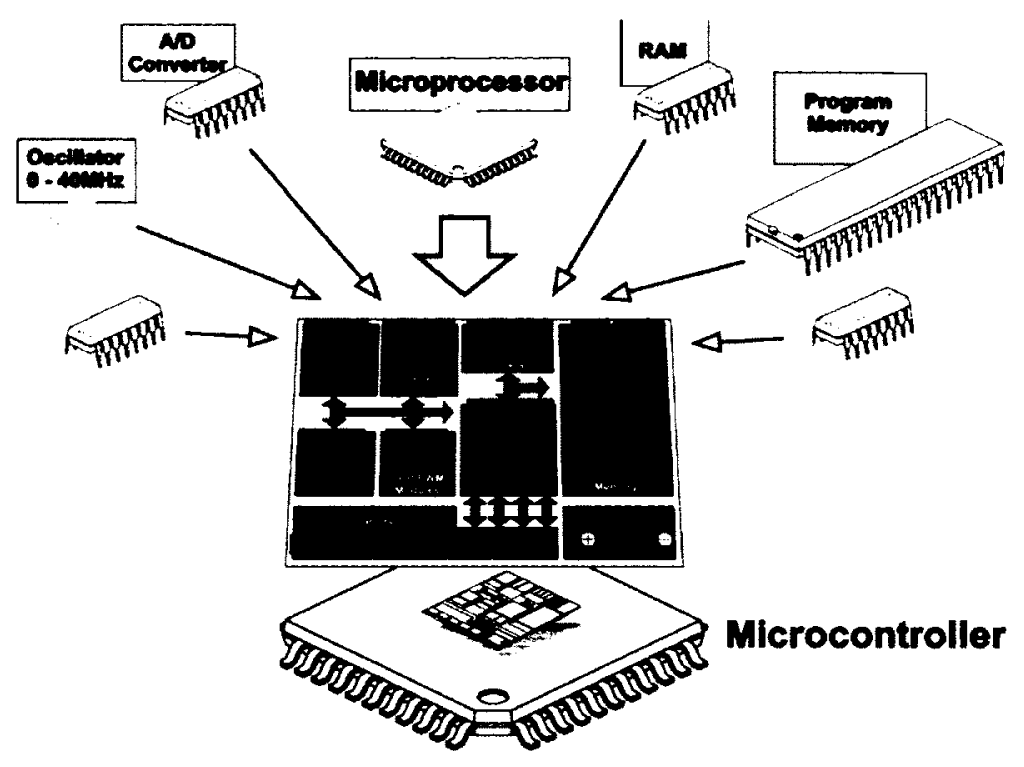

Figure 7.3: Microcontroller [69].

The brand of microcontroller that we used in this thesis is a series of microcontrollers called Peripheral Interface Controller (PIC) from Microchip manufacturer. This PIC microcontroller chip comes in different varieties, some with a basic low memory type, going all the way through analogue-to-digital converters and to even pulse-width modulation (PWM) built in. In this study, we started working with different PICs including: PIC 16F628, PIC 18F2520, PIC 16F876, and PIC 16F877, as illustrated in Figure 7.4.

- PIC 18F2520 is the newest part of the series of PICs we used. PIC 18F2520 is the chip with prefix 18 and is for 16-bit core chips. It has an improved instruction set, peripherals, and twice the code and the speed compared to 14-bit (16F) PICs. However, the price is slightly higher.

- PIC 16F628 is quite cheaper compared to the old 16F84; however, it has twice the code size, much more RAM, a UART and some more instruction sets. The chip is suitable to simple applications and learning. 
- PIC 16F876 is the microcontroller we chose for the study because of its size, memory, features of the chip, ease of use, quality, and cost. However, the chip has less code size, RAM, and I/O compared to the $16 \mathrm{~F} 877$. The price is the same as the PIC $16 \mathrm{~F} 877$.

- PIC 16F877 is the largest chip of the 16F87X family and a little bit expensive compared to PIC 16 F628 or 18 F2520; however, it has eight times the code size, much more RAM and much more I/O pin, a UART, A/D converter, and a lot more.

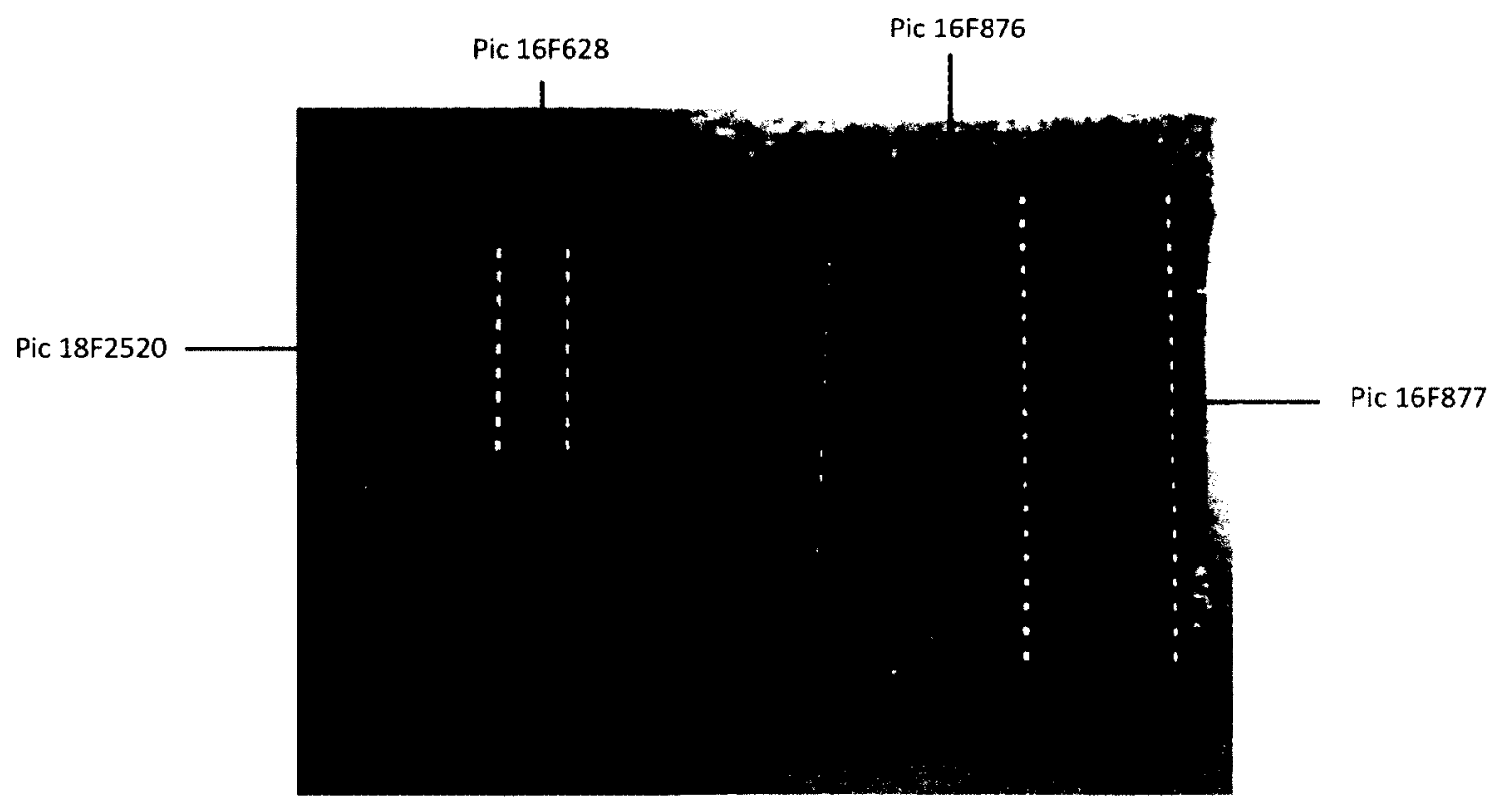

Figure 7.4: PIC 18F2520, PIC 16F628, PIC 16F876, and PIC 16 F877 


\section{Appendix D: Components}

This section provides a description of each component in detail. These components include sensors, $5 \mathrm{~V}$ regulator, and a buzzer. The sensors non-invasive consist of temperature, humidity, photocell, ultrasonic, pyroelectric ("passive"), Infrared (PIR), and Hall Effect. A more detailed explanation of each component is in the following subsections.

\section{V DC voltage regulator}

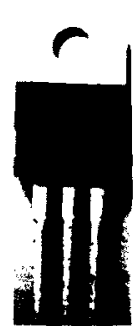

The most common 5V DC voltage regulator is called LM 7805. LM 7805 provides circuit designers with a simple way to regulate $D C$ voltages to $5 \mathrm{~V}$. Summarized in a single chip/package (IC), the LM 7805 is a positive voltage DC regulator that has only 3 terminals: Input voltage, Ground, and Output Voltage. LM 7805 has a power supply of 1 AMP and tolerates $+/-5 \%$, which means that the voltage of $5 \mathrm{~V}$ with a toleration of $+/-5 \%$ can yield result of between $5.25 \mathrm{~V}$ to $4.75 \mathrm{~V}$; this is a major concern for the accuracy of output for the sensors.

\begin{tabular}{|c|c|}
\hline Type & LM 7805CT-ND \\
\hline Voltage output & $5 \mathrm{~V} @ 1 \mathrm{~A}$ \\
\hline Operating Temperature & $-40^{\circ} \mathrm{C} \sim 125^{\circ} \mathrm{C}$ \\
\hline Manufacturer & Fairchild Semiconductor \\
\hline
\end{tabular}

Table 7.7: Characteristics of LM7805CT

\section{Piezoelectronic Buzzer}

The type of buzzer that was chosen for the research is called the piezoelectronic buzzer. The characteristics of the piezoelectronic buzzer are shown in Table 7.8. The device is used for many applications, including for making beeps, tones, and alerts. A piezoelectronic buzzer is small but loud. It operates with 3-30V peak-to-peak square waves. It has two pins; one pin can be connected as a ground and the other pin to a square wave out from a timer or microcontroller. The 
loudest tones can be achieved at a frequency around $4 \mathrm{KHz}$; however, a piezoelectronic buzzer operates quite well from $2 \mathrm{KHz}$ to $10 \mathrm{KHz}$. To get extra volume, both pins can be connected to a microcontroller and swapped to determine which of the pins is high or low for double the volume.

\begin{tabular}{|c|c|}
\hline Component type & PS1240P02BT \\
\hline Component size & $12.2 \mathrm{~mm} \times 6.5 \mathrm{~mm} \times 5 \mathrm{~mm}$ \\
\hline Sound pressure & $70 \mathrm{dBA}$ \\
\hline Operating temperature range & -10 to $70^{\circ} \mathrm{C}$ \\
\hline Storage condition & 5 to $40^{\circ} \mathrm{C}$ \\
\hline Maximum input voltage & $30 \mathrm{~V}$ \\
\hline Minimum input voltage & $3 \mathrm{~V}$ \\
\hline Manufacturer & TDK \\
\hline
\end{tabular}

Table 7.8: Characteristics of the piezoelectronic buzzer

\section{TMP36 Temperature Sensor}

The TMP36 temperature sensor is the type of sensor that was used for our research. The reason for choosing this temperature sensor is that this sensor has a very wide range. It produces temperature readings in Celsius and does not require a negative voltage to read sub-zero temperatures. It is inexpensive, small, and can take up very little space in our circuit board. The characteristics of the TMP36 temperature sensor are shown in Table 7.9. The temperature sensor is of an analog type. The sensor is an integrated circuit (IC) and does not require external calibration or trimming. Temperature is a low-voltage operation between $2.7 \mathrm{~V}$ to $5.5 \mathrm{~V}$ that calibrates directly in ${ }^{\circ} \mathrm{C}$ and $10 \mathrm{mV} /{ }^{\circ} \mathrm{C}$ scale factor. It has a $\pm 2^{\circ} \mathrm{C}$ accuracy over temperature and $\pm 0.5^{\circ} \mathrm{C}$ linearity. The sensor is stable with large capacitive loads, specified at $-40^{\circ} \mathrm{C}$ to $+125^{\circ} \mathrm{C}$, and operating to up $+150^{\circ} \mathrm{C}$.

\begin{tabular}{|c|c|}
\hline Sensor type & Analog Devices TMP36 \\
\hline Sensor size & $0.2^{\prime \prime} \times 0.2^{\prime \prime} \times 0.2^{\prime \prime}$ \\
\hline Temperature range & $-40^{\circ} \mathrm{C}$ to $150^{\circ} \mathrm{C} /-40^{\circ} \mathrm{F}$ to $302^{\circ} \mathrm{F}$ \\
\hline Output voltage range & $0.1 \mathrm{~V}\left(-40^{\circ} \mathrm{C}\right)$ to $2.0 \mathrm{~V}\left(150^{\circ} \mathrm{C}\right)$ \\
\hline Power supply & $2.7 \mathrm{~V}$ to $5.5 \mathrm{~V}$ only, $0.05 \mathrm{~mA}$ current draw \\
\hline Manufacturer & Analog Devices \\
\hline
\end{tabular}

Table 7.9: Characteristics of TMP36 temperature sensor 


\section{Humidity Sensor}

The humidity sensor that we used for the research is the CHS-GSS brand. The relative humidity

$(\mathrm{RH})$ for the sensor can be read directly with a voltmeter; output DC of $1 \mathrm{~V}$ can produce $100(\%)$

$\mathrm{RH}$. The sensor is capable of measuring humidity in the relative humidity range of $5 \%$ to $95 \%$.

It operates at a temperature range of $0^{\circ} \mathrm{C}$ to $50^{\circ} \mathrm{C}$, and requires a $5 \mathrm{~V}$ power supply. In addition, the sensor has low current consumption, only $0.6 \mathrm{~mA}$. Table 7.10 provides a brief description of the features for the humidity sensor that was used for the research.

\begin{tabular}{|c|c|}
\hline Sensor type & CHS-series \\
\hline Sensor size & $27 \mathrm{~mm} \times 11.5 \mathrm{~mm}$ \\
\hline Measuring range & $5 \%$ to $95 \%(\mathrm{RH})$ \\
\hline Operating condition & 0 to $50^{\circ} \mathrm{C}$ \\
\hline Storage condition & -20 to $60^{\circ} \mathrm{C}$ \\
\hline Power supply & $5 \mathrm{~V}$ \\
\hline Manufacturer & TDK \\
\hline
\end{tabular}

Table 7.10: Characteristics of humidity sensor

\section{Photocell sensor - light sensor}

The photocell sensor that we used for our research is also known as a cadmium-sulfide (Cds) cell.

The sensor is made of cadmium-sulfide; it is small, inexpensive, low-power, easy to use, and does not wear easily. It can be found in toys, gadgets, and appliances. However, in our research, we used the sensor to detect lightness and darkness in a room. Table 7.11 provides specifications of the photocell (light) sensor that was used for the purpose of this research.

\begin{tabular}{|c|c|}
\hline Sensor type & PDV-P8001 \\
\hline Sensor size & $5 \mathrm{~mm}\left(0.2^{\prime \prime}\right)$ diameter. \\
\hline $\begin{array}{c}\text { Operation } \\
\text { temperature range }\end{array}$ & $-20^{\circ} \mathrm{C}$ to $75^{\circ} \mathrm{C}$ \\
\hline Resistance range & 200K $\Omega$ (dark) to $10 \mathrm{~K} \Omega$ (10 lux brightness) \\
\hline Sensitivity range & $\begin{array}{c}\text { Cds cells respond to light between } 400 \mathrm{~nm} \text { (violet) and 600nm } \\
\text { (orange) wavelengths, peaking at about 520nm (green) }\end{array}$ \\
\hline Power supply & $\begin{array}{c}\text { Almost anything up to } 100 \mathrm{~V}, \text { uses less than 1mA of current on average } \\
\text { (depends on power supply voltage) }\end{array}$ \\
\hline
\end{tabular}




\begin{tabular}{l|l} 
Manufacturer & Advanced Photonix, Inc. \\
\hline
\end{tabular}

Table 7.11: Characteristics of the photocell sensor

\section{Hall Effect Sensor - US5881LUA}

The type of magnetic sensor that we used for the research is the Hall Effect. The sensor has discs with an approximate $1 / 2^{\prime \prime}$ diameter and $3 / 16^{\prime \prime}$ thickness. This type of sensor is useful for noncontact/waterproof type switches, position sensors, and rotary/shaft encoders. The sensor operates at $3.5 \mathrm{~V}$ to $24 \mathrm{~V}$ and it has three terminals: pin 1 is the input voltage pin (left pin), which is used to connect to the power; pin 2 is the ground pin (the middle pin); and pin 3 is the output voltage pin, which requires a $10 \mathrm{~K}$ pull up resistor and is connected to power. The Hall Effect (magnetic) sensor was used to detect whether a door was open or closed. The characteristics of Hall Effect sensor are shown in Table 7.12.

\begin{tabular}{|c|c|}
\hline Component type & Unipolar switch-US5881LUA \\
\hline Component size & $\frac{1}{2}$ diameterX3 $/ 16^{\prime \prime}$ thick discs \\
\hline Operating temperature range & -50 to $150^{\circ} \mathrm{C}$ \\
\hline Maximum input voltage & $24 \mathrm{~V}$ \\
\hline Minimum input voltage & $3.5 \mathrm{~V}$ \\
\hline Output type & Digital \\
\hline Manufacturer & Melexis Inc. \\
\hline
\end{tabular}

Table 7.12: Characteristics of the Hall Effect sensor

\section{Ultrasonic Sensor}

The LV-EZ1 Maxbotix Ultrasonic Rangefinder was the type of ultrasonic sensor that we used for the

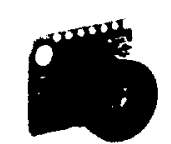
purpose of our research. The sensor provides very short- to long-range detection. It can detect objects from 0 -inches to 254 -inches (6.45-meters) and provides sonar range information from 6 -inches out to 254 -inches with 1 -inch resolution. The interface output formats included pulse width output (PWM), analog voltage output ( $\mathrm{Ccc} / 512$ volts per inch), and serial digital output (9600 baud) [48]. The analog output is used to determine the distance of the object. An output 
analog voltage with a scaling factor of $(\mathrm{Vcc} / 512)$ per inch and a supply of $5 \mathrm{~V}$ yields $\sim 9.8 \mathrm{mV} / \mathrm{in}$. The output is buffered and corresponds to the most-recent range data.

The benefits of the LV-EZ1 Maxbotix Ultrasonic Rangefinder include, but are not limited to, the following [70]:

- Acoustic and electrical noise resistance;

- Reliable and stable range data;

- Sensor dead zone virtually gone;

- Low cost;

- Quality controlled beam characteristics;

- Very low power range, excellent for multiple sensor or battery based systems;

- Can be triggered externally or internally;

- Sensor reports the range reading directly, frees up user processor;

- Fast measurement cycle;

- User can choose any of the sensor outputs;

- No calibration requirement, which is perfect for when objects may be directly in front of the sensor during power up; and

- Small size allows for easy mounting.

Table 7.13 provides a brief description of specifications for the ultrasonic sensor used for this research.

\begin{tabular}{|c|c|}
\hline Sensor type & LV - MaxBotix - EZO \\
\hline Sensor size & $0.645^{\prime \prime} \times 0.610^{\prime \prime}$ \\
\hline $\begin{array}{c}\text { RS232 serial } \\
\text { output }\end{array}$ & $9600 \mathrm{pbs}$ \\
\hline Analog output & $10 \mathrm{mV} /$ inch \\
\hline PWM output & $147 \mu \mathrm{s} /$ inch \\
\hline Power supply & $2.5 \mathrm{~V}-5.5 \mathrm{~V}$ \\
\hline Manufacturer & MaxBotix \\
\hline
\end{tabular}

Table 7.13: Characteristics of ultrasonic sensor 


\section{Appendix E: Power Supply}

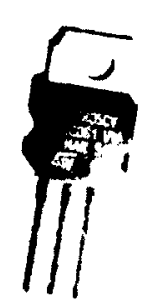

In many countries, the house outlet operates at a power of $220 \mathrm{~V} \mathrm{AC} \mathrm{(Alternating} \mathrm{Current),}$ while in North America or in Canada the house outlet operates at a power of $110 \mathrm{~V} \mathrm{AC}$. Alternating current is very bad for $5 \mathrm{~V} \mathrm{DC}$ components. Therefore, the $110 \mathrm{~V} A C$ or $220 \mathrm{AC}$ must be converted from a house outlet to a useable $5 \mathrm{~V} \mathrm{DC}$. In order to convert the alternating current from the house outlet to a useable $5 \mathrm{~V} \mathrm{DC}$, voltage regulators are required. The most common $5 \mathrm{~V}$ DC voltage regulators are called LM 7805 with a power supply of 1 AMP with a toleration level of $+/-5 \%$, which means the voltage of $5 \mathrm{~V}$ with a toleration level of $+/-5 \%$ can yield results between $5.25 \mathrm{~V}$ to 4.75 $V$, which is a major concern for the accuracy of the output for the sensors. LM 8705 is illustrated in Figure 7.8. LM 7805 provides circuit designers with a simple way to regulate DC voltages to $5 \mathrm{~V}$. Summarized in a single chip/package (IC), the $L M 7805$ is a positive voltage DC regulator that has only 3 terminals: input voltage, ground, and output voltage.

Even though the LM 7805s were mainly designed for a fixed-voltage output (5V), it is in fact possible to use external components in order to obtain DC output voltages of: $5 \mathrm{~V}, 6 \mathrm{~V}, 8 \mathrm{~V}, 9 \mathrm{~V}, 10 \mathrm{~V}, 12 \mathrm{~V}, 15 \mathrm{~V}, 18 \mathrm{~V}$, $20 \mathrm{~V}$, and $24 \mathrm{~V}$. Note that the input voltage must, of course, be greater than the mandatory output voltage, so that it can be regulated downwards.

\section{General Features:}

- Output current up to $1 \mathrm{~A}$

- Output voltages of $5 \mathrm{~V}$ or higher

- Thermal overload protection

- Short circuit protection

- Output transistor safe operating area protection 
For the accuracy of the output voltage, the noise coming in the input pin must be reduced. The easiest way to help smooth out ripples is by using filtering capacitors. Electrolytic capacitors and ceramic capacitors are the two types of capacitors we used to build the voltage regulator circuit.

Electrolytic capacitors: The two examples of electrolytic capacitors are illustrated in Figure 7.5. The capacitors have storage of $10 \mu \mathrm{F} / 50 \mathrm{~V}$, which means it can withstand up to $50 \mathrm{~V}$. The capacitors with storage of $100 \mu \mathrm{F} / 16$ can withstand up to $16 \mathrm{~V}$. They are polarized with a positive pin and a negative pin. The positive pin is a little bit longer than the negative pin. The positive pin is connected to the input and $10 \mu \mathrm{F} / 16 \mathrm{~V}$ is connected to the output. The negative pin is connected to ground.

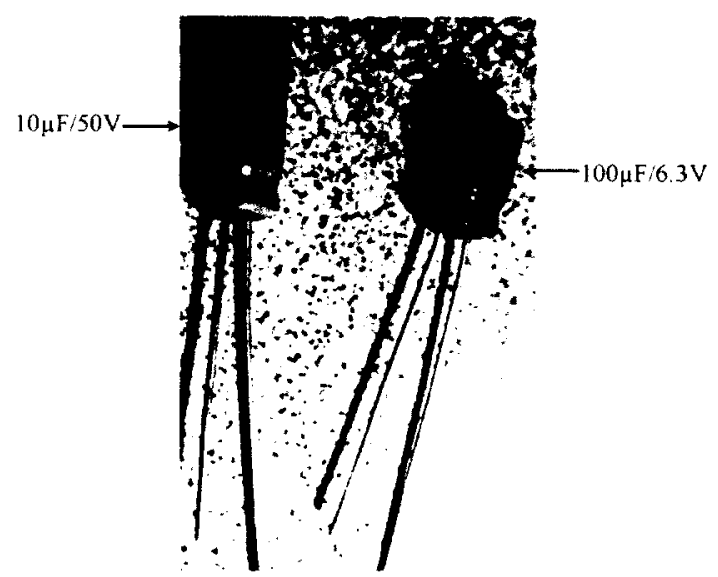

Figure 7.5: Electrolytic capacitors $(10 \mu \mathrm{F} / 50 \mathrm{~V})$ and ceramic capacitor $(100 \mu \mathrm{F} / 6.3 \mathrm{~V})$

Ceramic capacitors: are non-polarized capacitors operating at a value of $100 \mu \mathrm{F} / 6.3 \mathrm{~V}$. It has 2 pins and is not positive or negative. Therefore, the pins can be connected in the breadboard in any way.

Both capacitors are required to build the $5 \mathrm{~V}$ power supply because electrolytic capacitors are larger capacitors and are very slow in releasing their stored energy, while ceramic capacitors are small and very fast in the delivery of their stored energy. So electrolytic capacitors can help maintain a declining voltage while ceramic capacitors can help contain higher frequency noise and shorter power dips. 
Capacitors cannot deliver their stored energy instantaneously. Larger caps (10uF and 100uF) store more energy, but they react more slowly. The smaller the capacitor, the faster it can deliver its stored energy. If you have a large power outage (power dips for $10-100 \mathrm{~ms}$ ), a big cap (100uF to 1000uF) will help 'hold up' the falling voltage. A smaller cap (0.1uF) will help suppress higher frequency noise and shorter power dips (noise in the 1us to 100us range). Therefore, 0.1 uF caps are located near the microcontroller to help with short bursts, where 100uF and 10uF caps are used on the power rails [71].

\section{Battery}

The battery supplies power to the remote station. It is a 5 voltage power source for the circuit. The power can be supplied to the remote system through $9 \mathrm{~V}$ regulated DC wall-power adapter or $9 \mathrm{~V}$ battery.

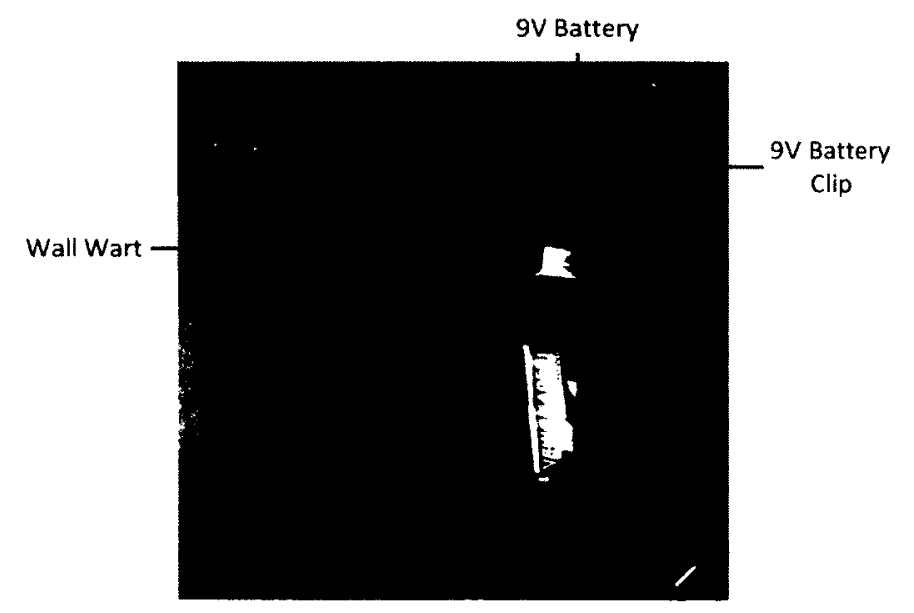

Figure 7.6: Battery with $9 \mathrm{~V}$ regulated $\mathrm{DC}$ wall-power adapter and $9 \mathrm{~V}$ battery clip 


\section{Appendix F: Testing}

The testing sensors steps are as follows:

- Testing Temperature Sensor: The temperature sensor that we used for the study comes in a "TO-92" package, which means that the chip is housed in a plastic hemi-cylinder with three pin terminals: input, output, and ground. The pins can be bent easily to allow the sensor to be plugged into a breadboard or can also be soldered to the pins to connect long wires. Figure 7.7 (Figure 8.3 A) illustrates the temperature sensor that was used for the study.

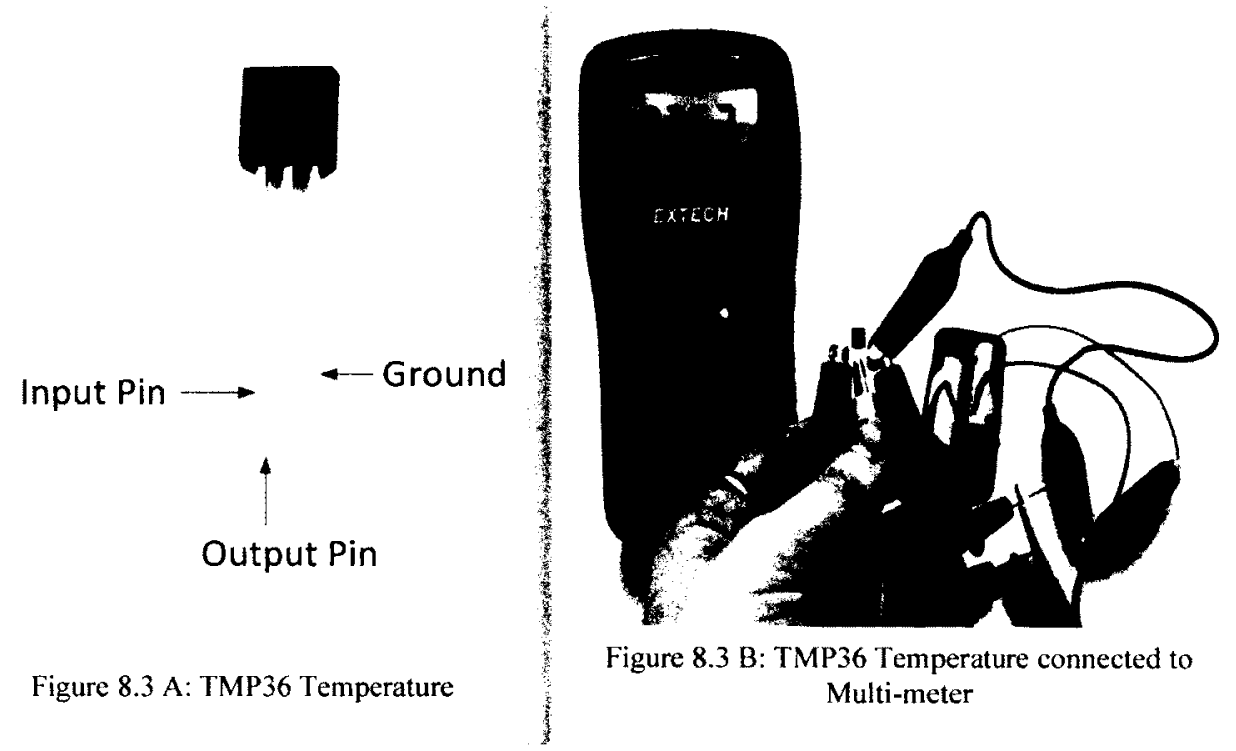

Figure 7.7: TMP36 Temperature ${ }^{19}$

We tested the temperature sensor using different techniques and tools such as a soldering iron, air conditioner, and an ice cube.

\footnotetext{
${ }^{19}$ Figure $8.3 \mathrm{~B}$ is from Adafruit website, source: http://www.ladyada.net/learn/sensors/tmp36.html
} 
- Testing Temperature sensor using soldering iron: The soldering iron that we used to test

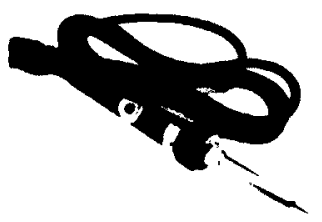
temperature level of the sensor is a "pen-style" soldering iron; this soldering iron is shown to the right of the text. The plug for the iron is 110 US-style and can only be used in $110 \mathrm{~V}$ countries, such as USA and Canada. The iron also comes with a very simple flip-up stand, which is only marginally useful.

- Testing the functionality of Temperature sensor: Once the soldering iron was plugged in and heated up, it was placed close to but not as close to touch the sensor, so that it could not cause the sensor to melt. The heating soldering iron allows the temperature to increase gradually; through multi-meter and LCD we observed that the temperature reading was increasing gradually. However, we stopped raising the temperature when it reached 30 degrees Celsius because we set a buzzer to go off at that temperature.

- In addition to using a soldering iron to test the functionality of the sensor, we also held the sensor using our fingers as shown on figure 7.8 (Figure $8.5 \mathrm{~A}$ ) to observe any change in the temperature.

- We also used a piezo buzzer set to go off when the temperature reading reached 30 degrees Celsius or higher and also when the temperature reading dropped below 15 degrees Celsius.

- Testing Temperature sensor using an ice cube: The other testing we used was an ice cube, as illustrated in figure 7.8 (Figure 8.5B). We pressed the ice cube against the sensor. The ice cube was carefully covered by a plastic bag to prevent water from getting on the circuits. We observed over the multi-meter that the temperature was dropping gradually. 


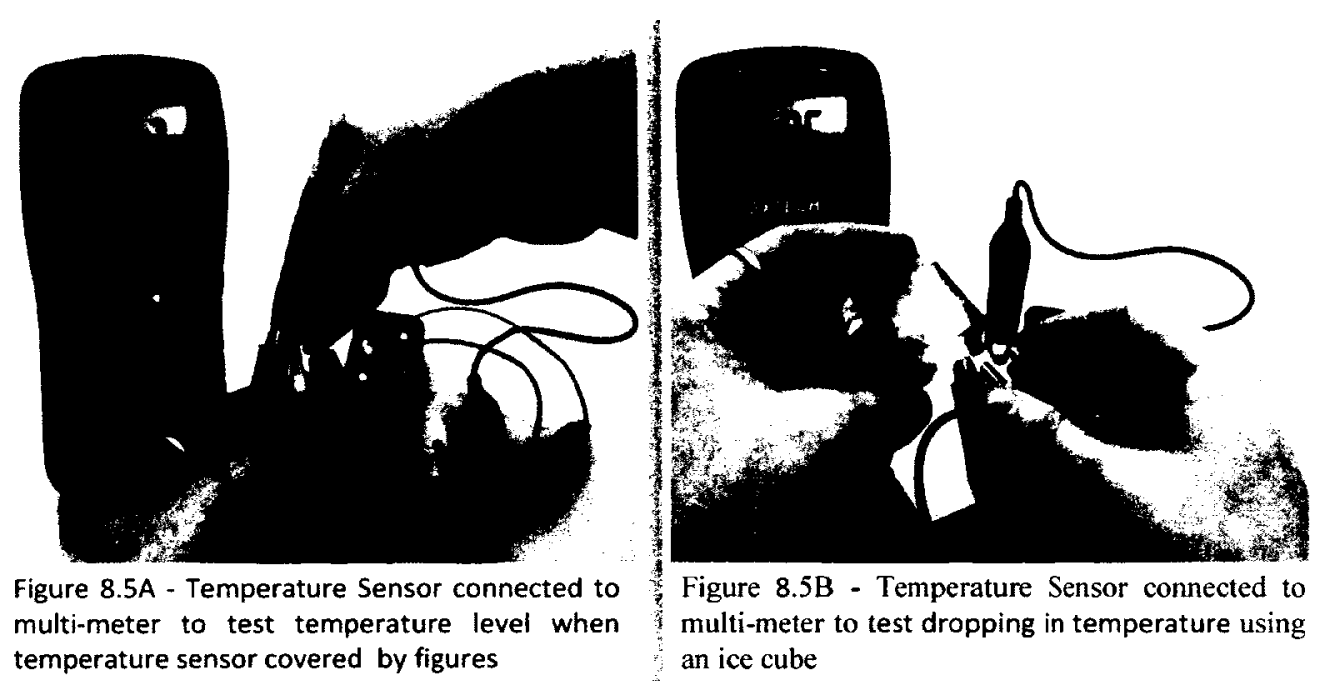

Figure 7.8: Testing Temperature Sensor using an ice cube 20

- Testing temperature sensor after connection: After connecting the component, we applied the same above testing to the sensor to determine its functionality. Not only were we able gather data using the multi-meter, but we were also able to use LCD and X-CTU software to test the sensor this time.

- Testing Photocell (Light) Sensor: The sensor we used for the research is non-polarized just like basic resistors. It has a two-pin terminal that can be connected in measurement mode to the two leads up in "either way" of pins to a multi-meter in resistance, as illustrated in Figure 7.9 (Figure 8.7A). We then observed on the multi-meter how the resistance changes when shading the sensor with our hand or turning on/off lights of the room. This light sensor test indicated the functionality level of the sensor.

- Testing Photocell (black object) Sensor:

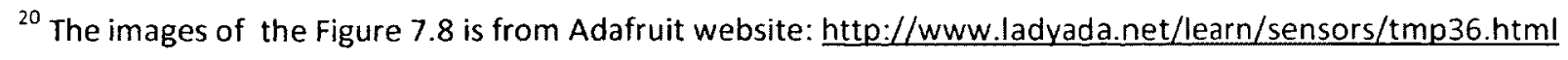


- The test was also done by covering the sensor with a black object, such as shown in Figure 7.9 (Figure 8.7B). Since the multi-meter resistance changes a lot, we used an auto-ranging meter. It worked well here. Different ranges of resistances between $1 \mathrm{M} \Omega$ and $1 \mathrm{~K} \Omega$ were also used to test the functionality of the sensor.

- Testing Photo cell (Light) sensor after connection

- The rest of the sensors: The remaining sensors are Ultrasound, Hall Effect, (Magnetic) motion, and humidity sensor and were tested by the connection of the System. Once connected, the same testing was done to the sensor to determine its functionality.

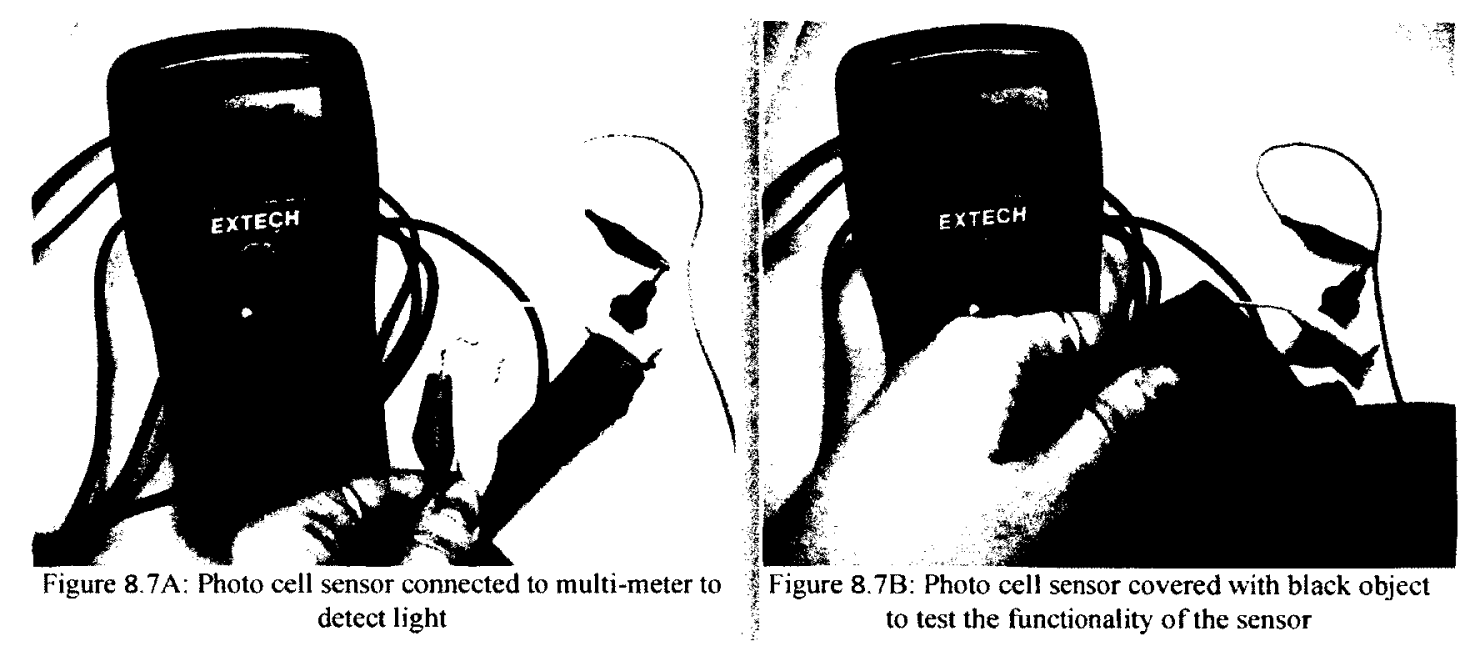

Figure 7.9: Detecting light using multi-meter ${ }^{21}$

${ }^{21}$ Source of the Figure 7.9 is from Adafruit website: http://www.ladyada.net/learn/sensors/tmp36.html 


\section{Appendix G: Proton IDE}

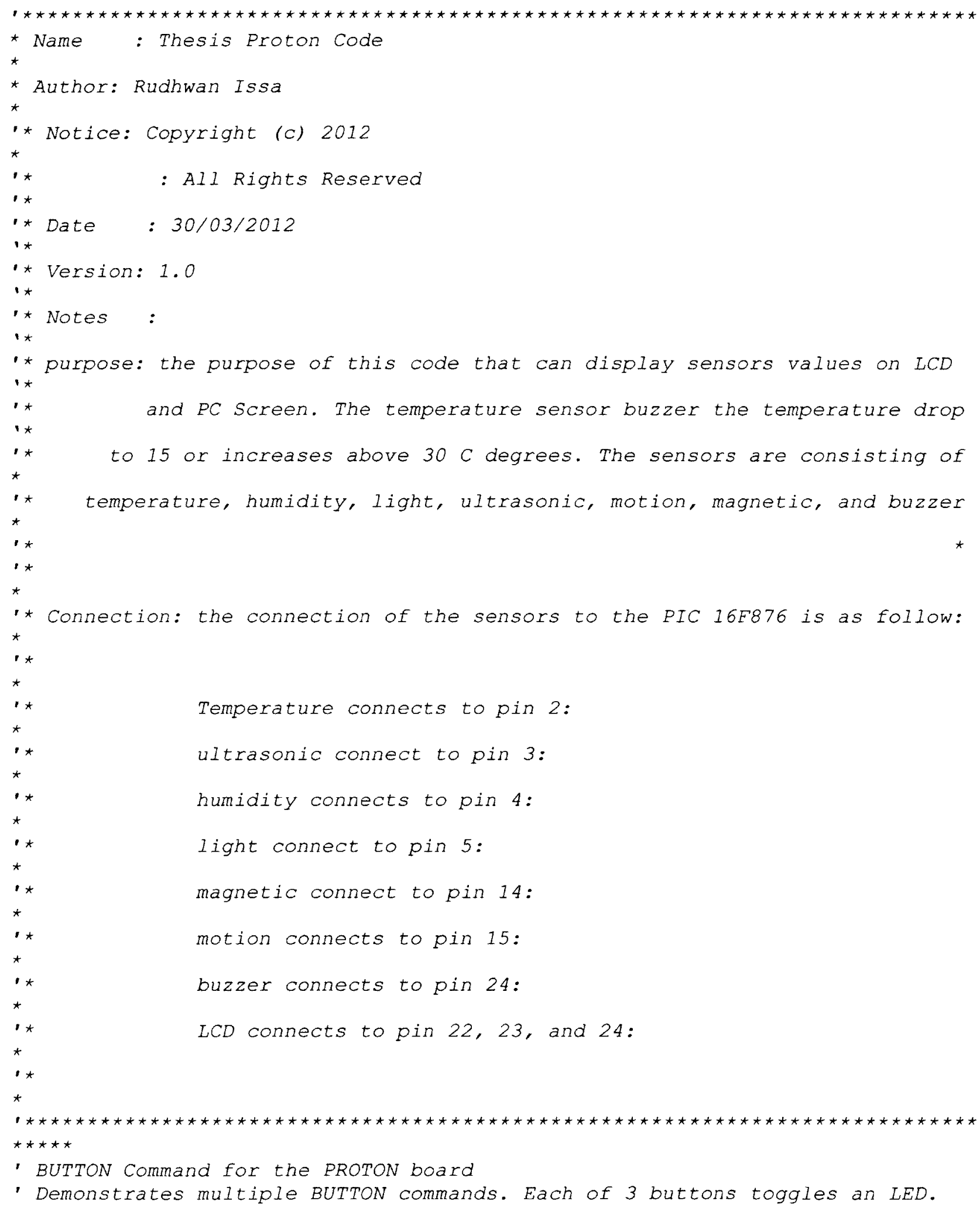


- Hold a button for 1 second and the LED will flicker (auto-repeat).

Device $=16$ F876

$\mathrm{XTAL}=20$

ALI_DIGITAL $=$ TRUE

TRISC $=\frac{8}{8} 11111110$

TRISB $=\frac{8}{8} 00001000$

TRISA $=\frac{8}{2} 11111111$

$A D C O N 1=\frac{8}{8} 10000010$

ADIN RES 10

ADIN TAD FRC

ADIN STIME 100

Print $\mathrm{Cls}$
'Select Microcontroller

'Select Clock frequency $20 \mathrm{MHz}$

'Make All lines digital as we are not

'going to use Analog

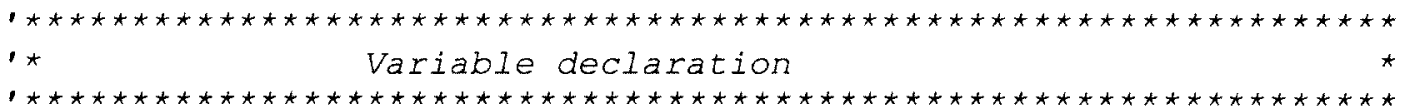

Symbol led $=$ PORTC.0

Dim raw As Word

- create an 32-bit unsigned variable

Dim raw1 As Word

'(0 to 65535)

Dim raw2 As Word

- (0 to 65535)

Dim raw4 As Word

- create an 32-bit unsigned variable ' (0 to 65535)

- create an 32-bit unsigned variable ' (o to 65535)

Dim $v$ As Float

- create an 32-bit floating point variable

Dim light As Float

- create an 32-bit floating point variable

Dim RH As Float

- create an 32-bit floating point variable

Dim distance As Float

- create an 32-bit floating point variable

PORTB_PULLUPS $=$ Off

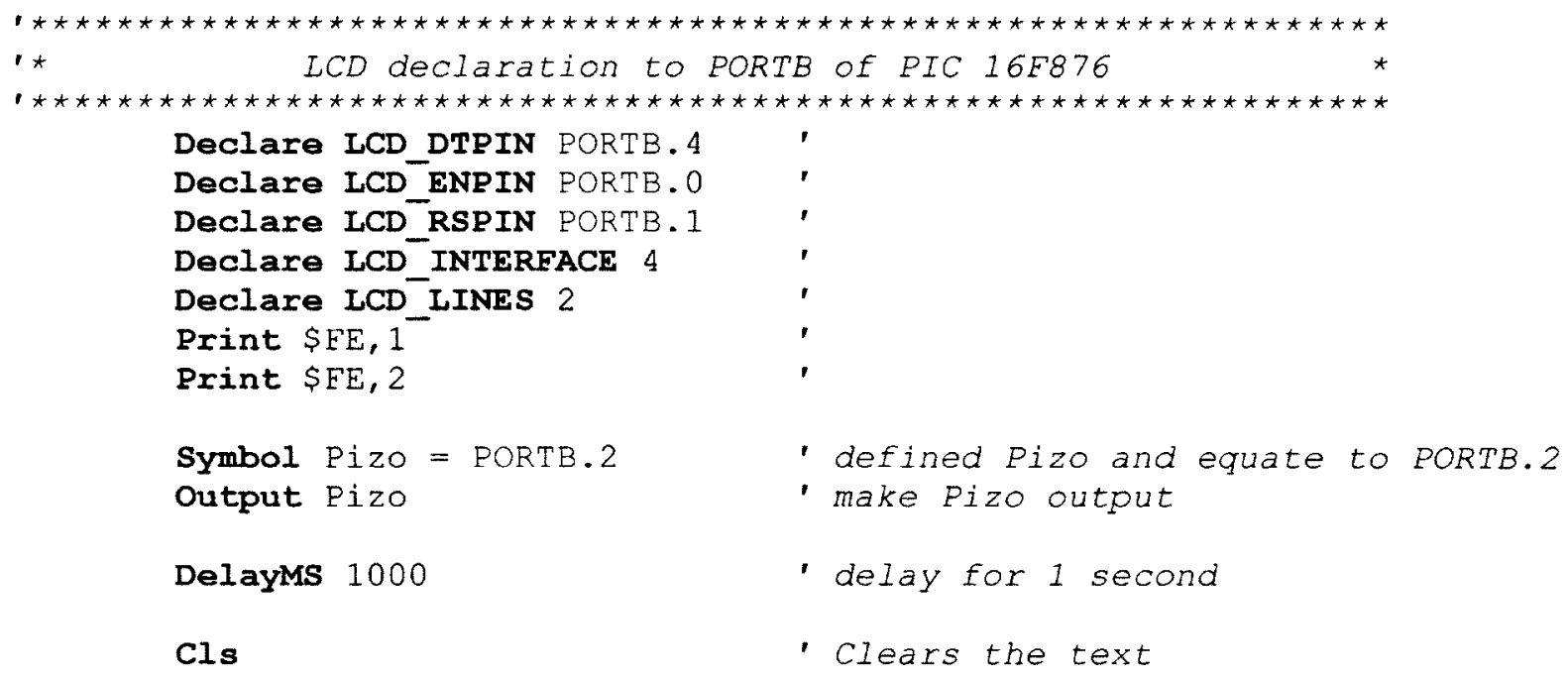




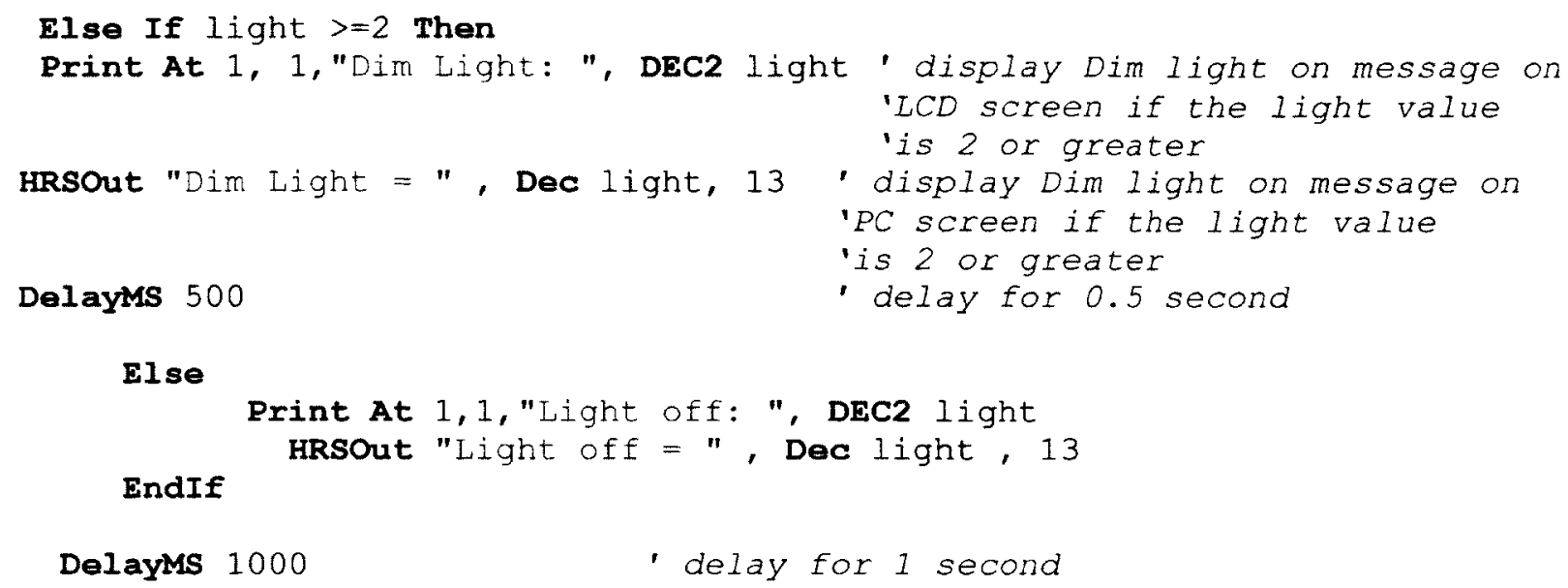

Else

Print At 1,1,"Light off: ", DEC2 light

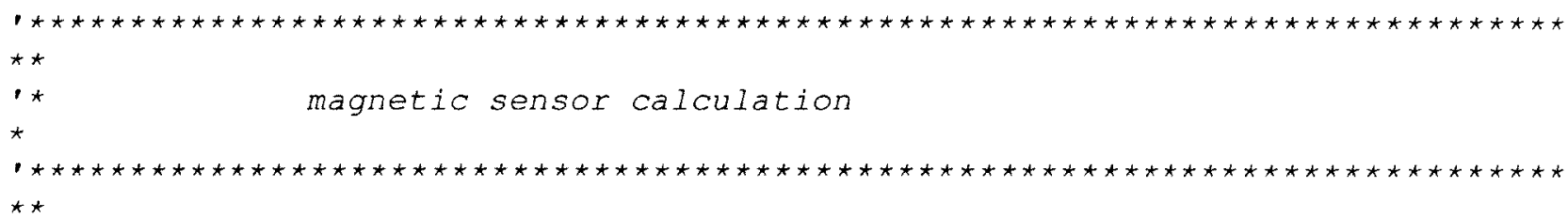

DelayMs 1000

'delay for 1 second

If PORTC. $3=0$ Then

Print At 1, 1," Door is Locked.." 'display "door is locked"

HRsout "Door is Locked..", 13

'message on LCD screen

- display "Door is locked

DelayMs 500

'message on PC screen

Else

Print At 1,1," Door is unlocked.."

'display "door is unlocked" 'message on LCD screen

HRSOut "Door is unlocked.. ", 13

- display "Door is unlocked"

Delayms 500

'message on PC screen

'delay for 0.5 second

Print $\$ F E, 1$

EndIf

Print $\$ F E, 2$

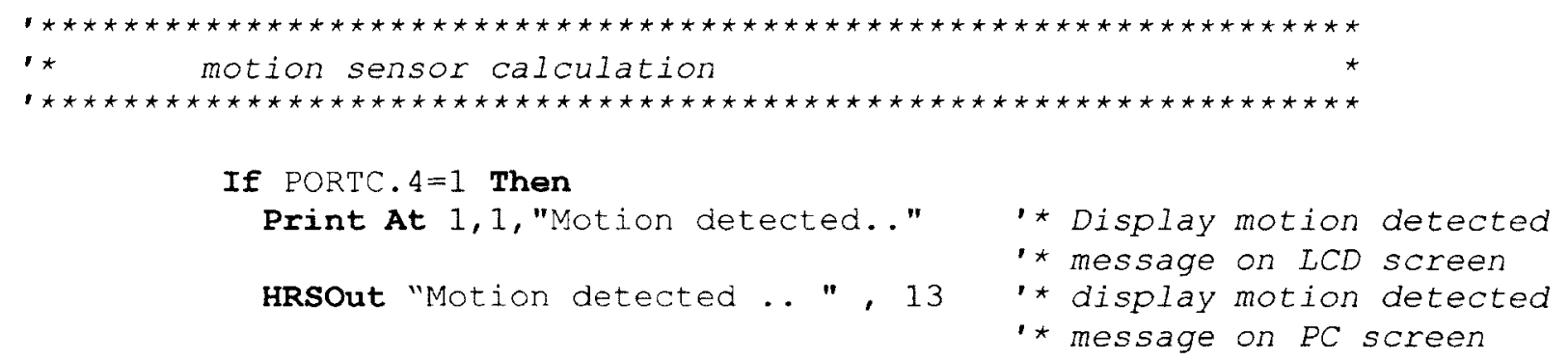


Print $S E E, 1$

Print $\$ F E, 2$

\section{EndIf}

GoTo loop End 


\section{Appendix H: Matlab}

\begin{tabular}{|c|c|c|c|}
\hline 差 & Title & : & Thesis Matlab Code to analyses collected data at \\
\hline 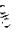 & & : & prototype apartment \\
\hline 实 & & : & \\
\hline E & Author & : & Rudhwan Issa \\
\hline$f$ & & : & \\
\hline ? & University & : & Carleton University \\
\hline 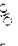 & & : & \\
\hline 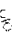 & Notice & : & Copyright (c) 2012 Rudhwan \\
\hline 8 & & : & All right reserved \\
\hline E & & : & \\
\hline 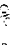 & Date & : & 30 February 2012 \\
\hline 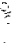 & & : & \\
\hline 5 & Notes & : & The goal of the Thesis Matlab code is to analyses our \\
\hline 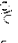 & & $:$ & collected at different locations (washroom, kitchen, \\
\hline$\frac{5}{5}$ & & : & living room, and balcony) and appliances (fridge, \\
\hline & & : & stove, and other appliances) of our prototype apartmer \\
\hline
\end{tabular}

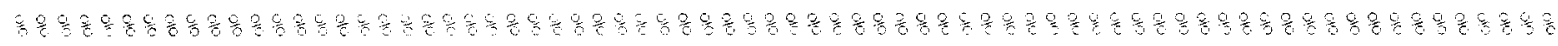
Deration for the collecting the data

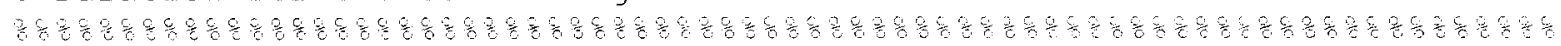
है을

Number $=[1: 1: 5]$;

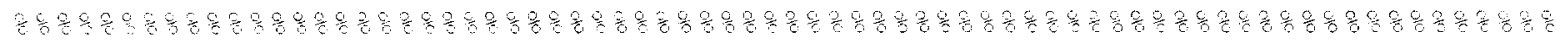
t Temperature Data for washroom, Eridge, kitchen, living room, and ts s outside at balcony of the apartment

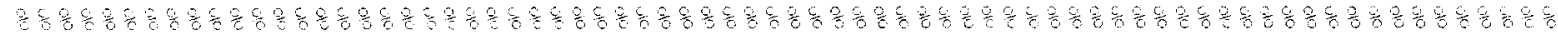
Temperature $=[23.802,25.268,22.825,24.780,22.825]$; washroom Temp Temperature $1=[3.274,11.584,7.673,11.584,8.651] ;$ : fridge Temp Temperature2 $=[22.825,24.780,26.246,25.757,26.409] ; \xi$ kitchen Temp Temperature3 $=[23.313,6.695,23.802,-3.567,22.825] ;$; living room Temperature $4=[-2.101,-4.545,4.740,4.578,-4.545] ;$ s outside Temp

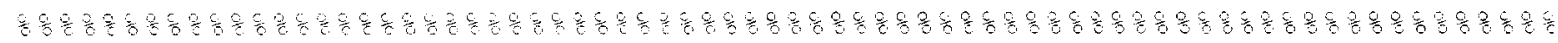
Humidity Data for washroom, fridge, kitchen, living room, and outsideg at balcony of the apartment

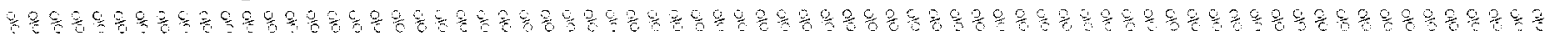
Humidity $=[26.392,86.532,32.258,80.156,22.482]$; , shower Humidity Humidity $1=[8.797,46.432,10.263,52.7858 .388] ; \quad$ S fridge Humidity Humidity2 $=[23.460,23.949,25.904,28.572,23.949]$; ? kitchen Humidity Humidity $3=[25.904,43.010,31.280,41.544,24.926] ;$ g living room Humidity $4=[76.246,51.808,67.937,44.477,43.988] ; \quad$ outside Humid

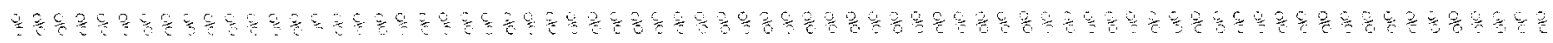

Light Data for washroom, fridge, kitchen, living room, and outside ats balcony of the apartment

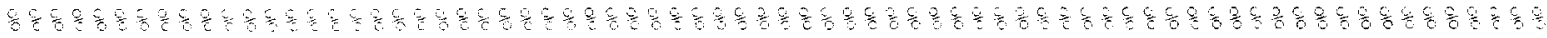
Light $=[0.03,0.03,3.07,3.07,0.02] ;$ sight data at washroom Light $1=[0.06,0.06,4.46,4.48,0.06] ; \quad$ Light data at fridge
} 


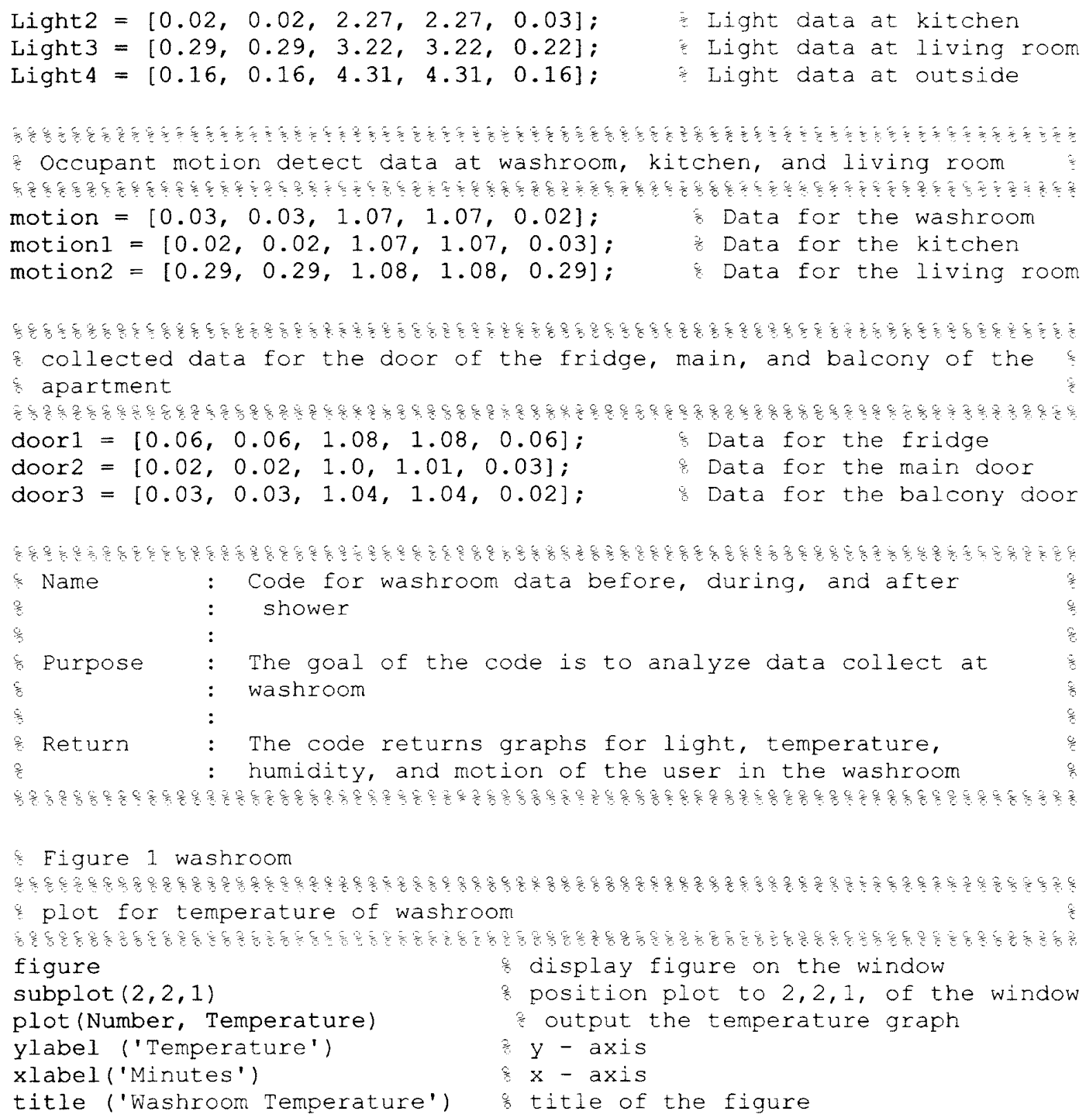
hold on

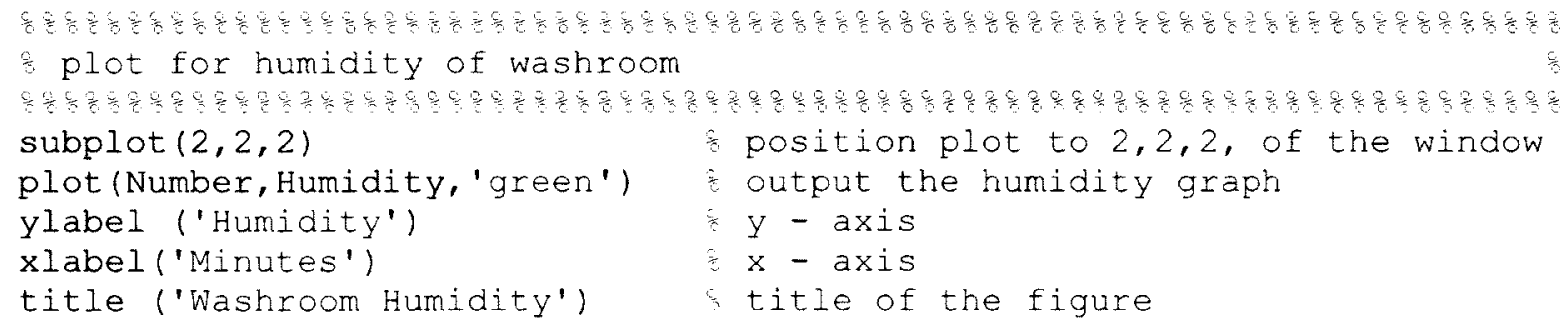
hold on 


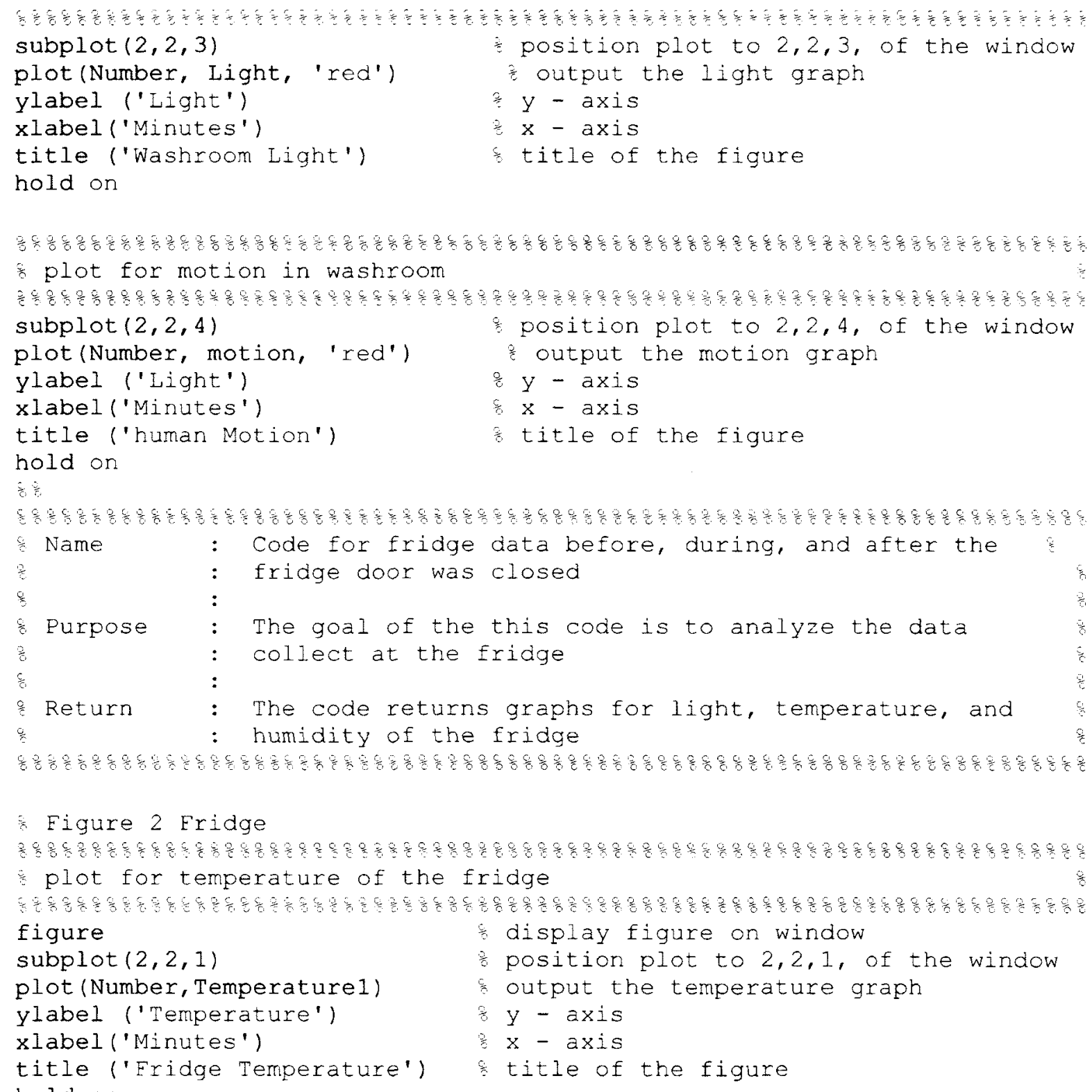
hold on

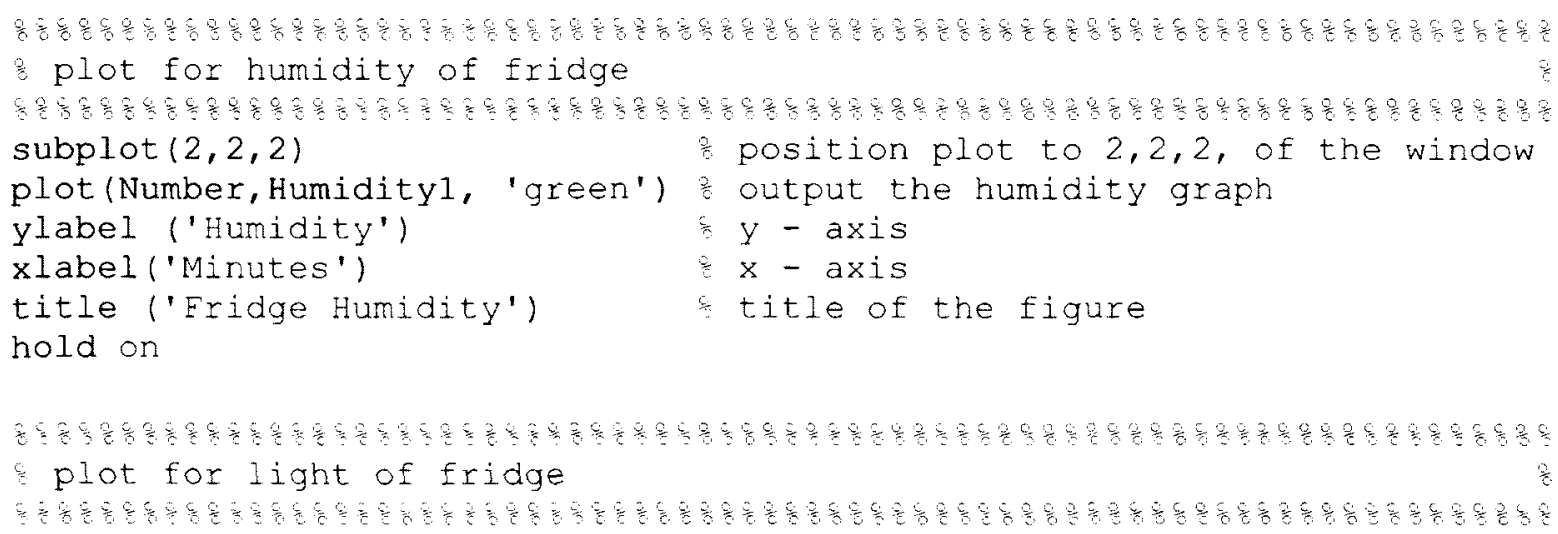




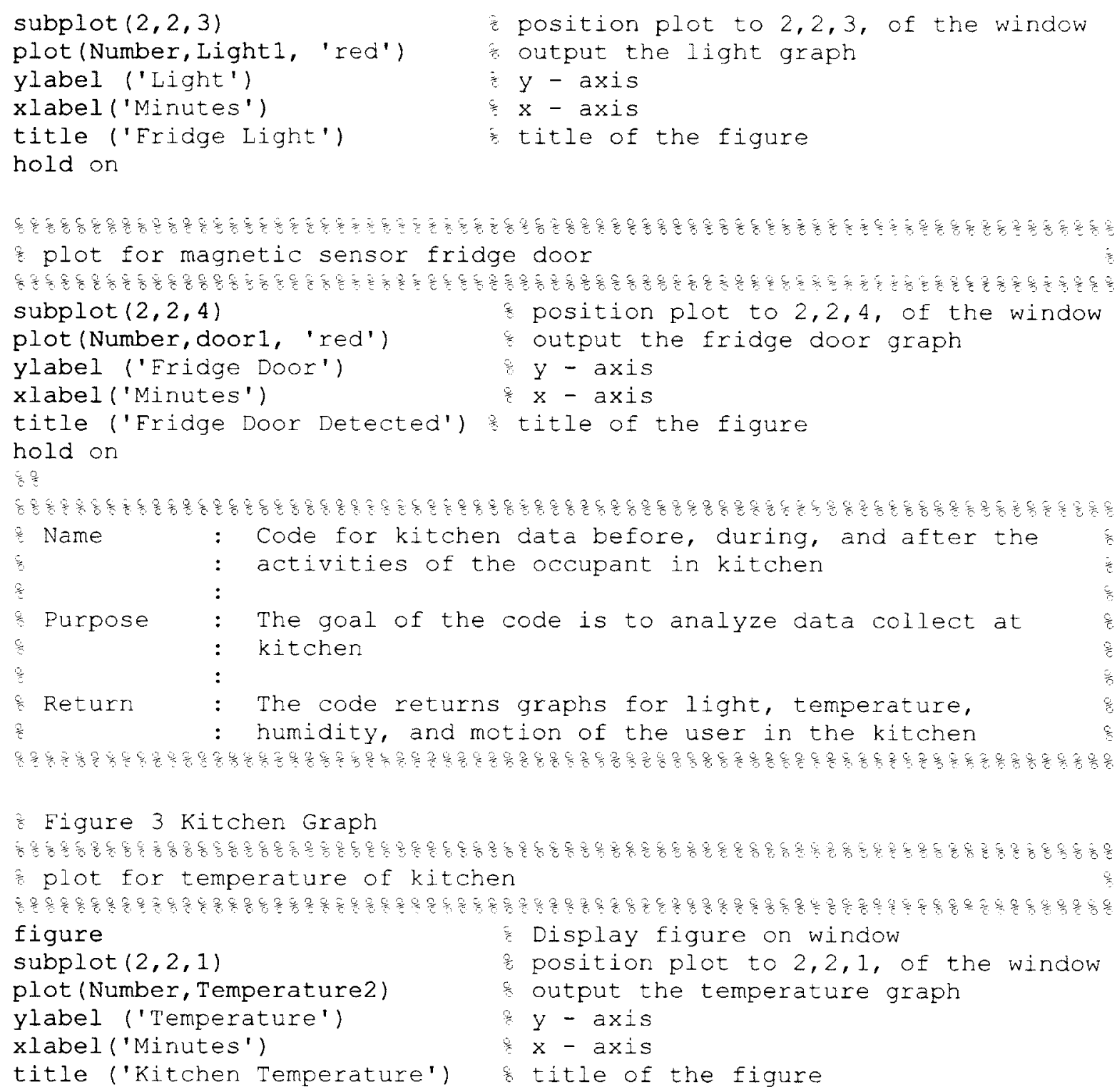
hold on

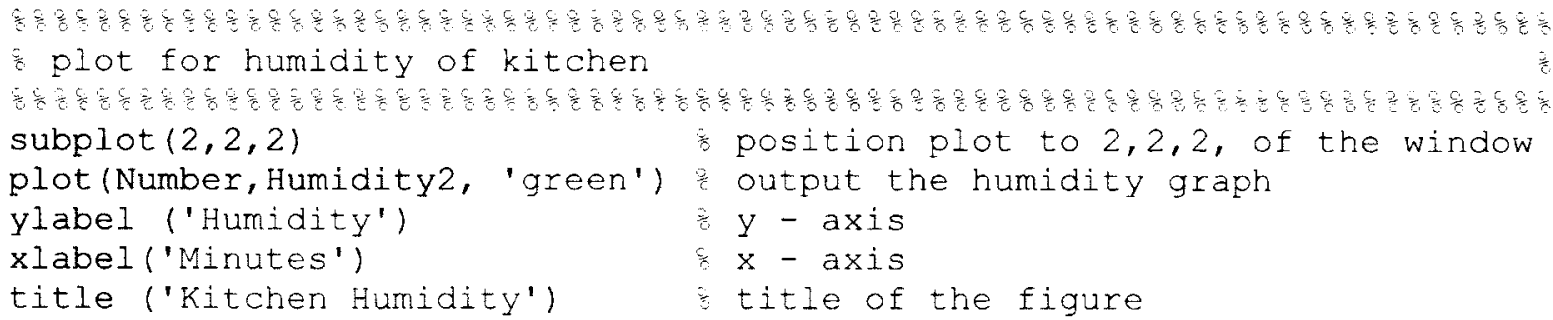
hold on

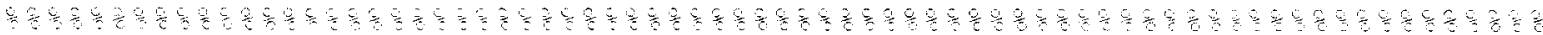
plot for light of kitchen

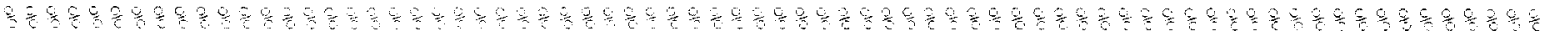
subplot $(2,2,3)$

position plot to $2,2,3$, of the window 


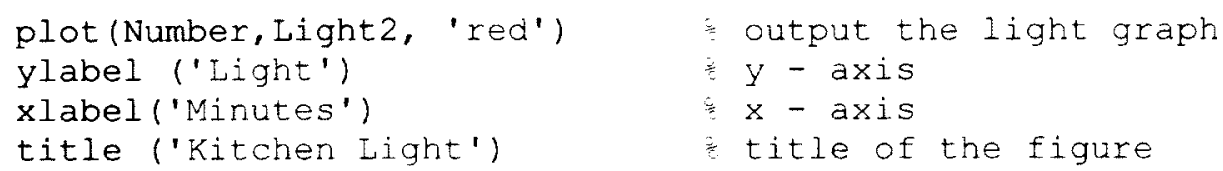

hold on

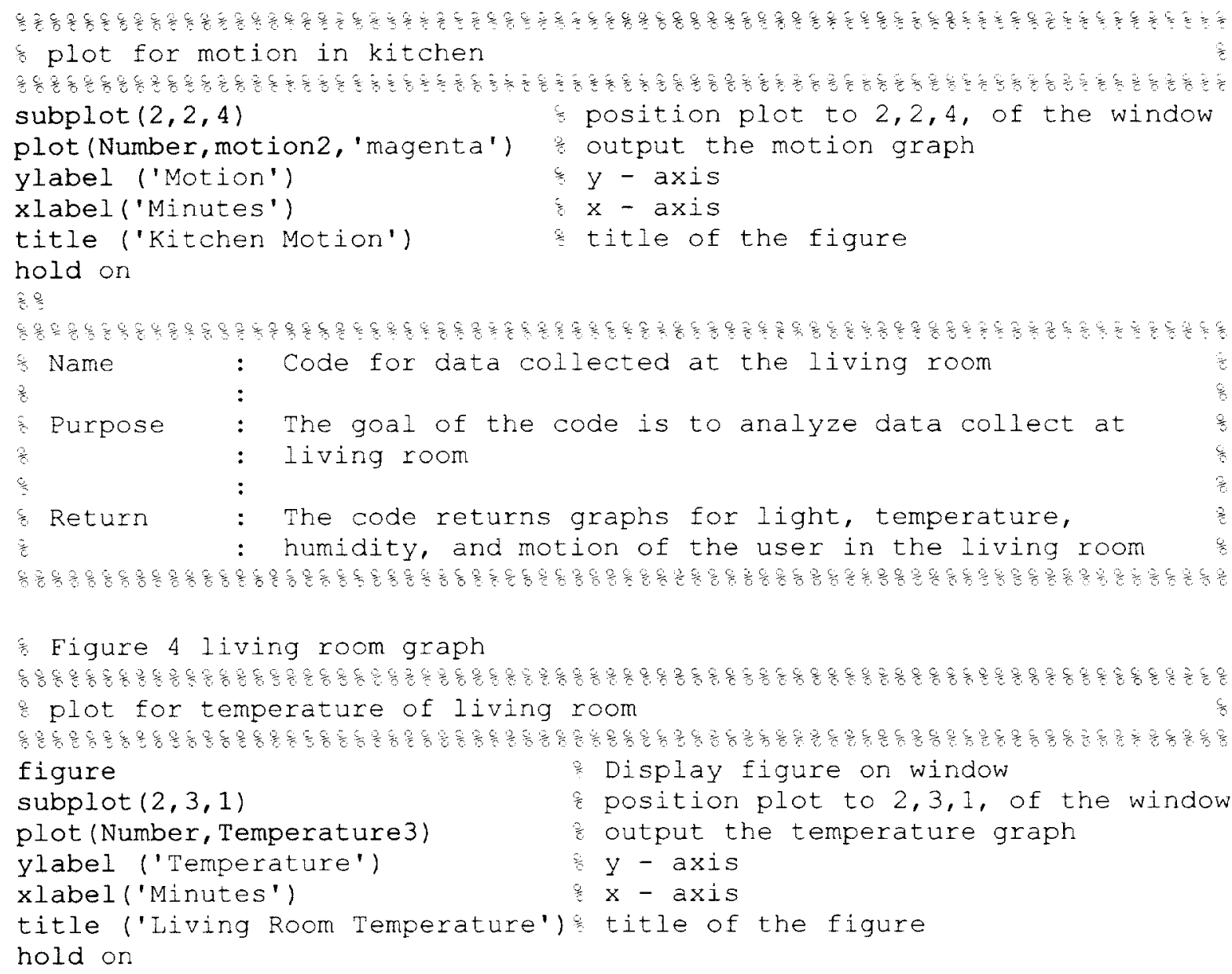




xlabel ('Minutes')
title ('Living Room Light') $\quad$ title of the figure
hold on

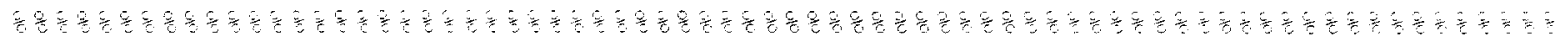

plot for magnetic sensor data balcony was open

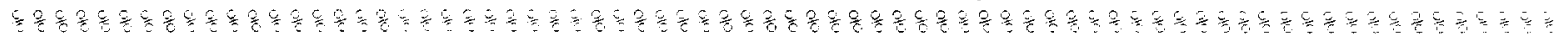

subplot $(2,3,4) \quad$ position plot to $2,3,4$, of the window

plot (Number, door2, 'red') output the balcony door graph

ylabel ('balcony door') $y$ - axis

xlabel ('Minutes')

$x-$ axis

title ('Living Room Balcony') y title of the figure

hold on

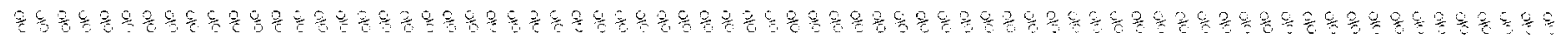

plot for motion in living room

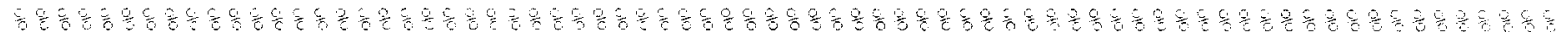

subplot $(2,3,5)$

plot (Number, motion2, 'red')

ylabel ('motion')

xlabel ('Minutes')

position plot to $2,3,5$, of the window

title ('Living Room motion')

hold on

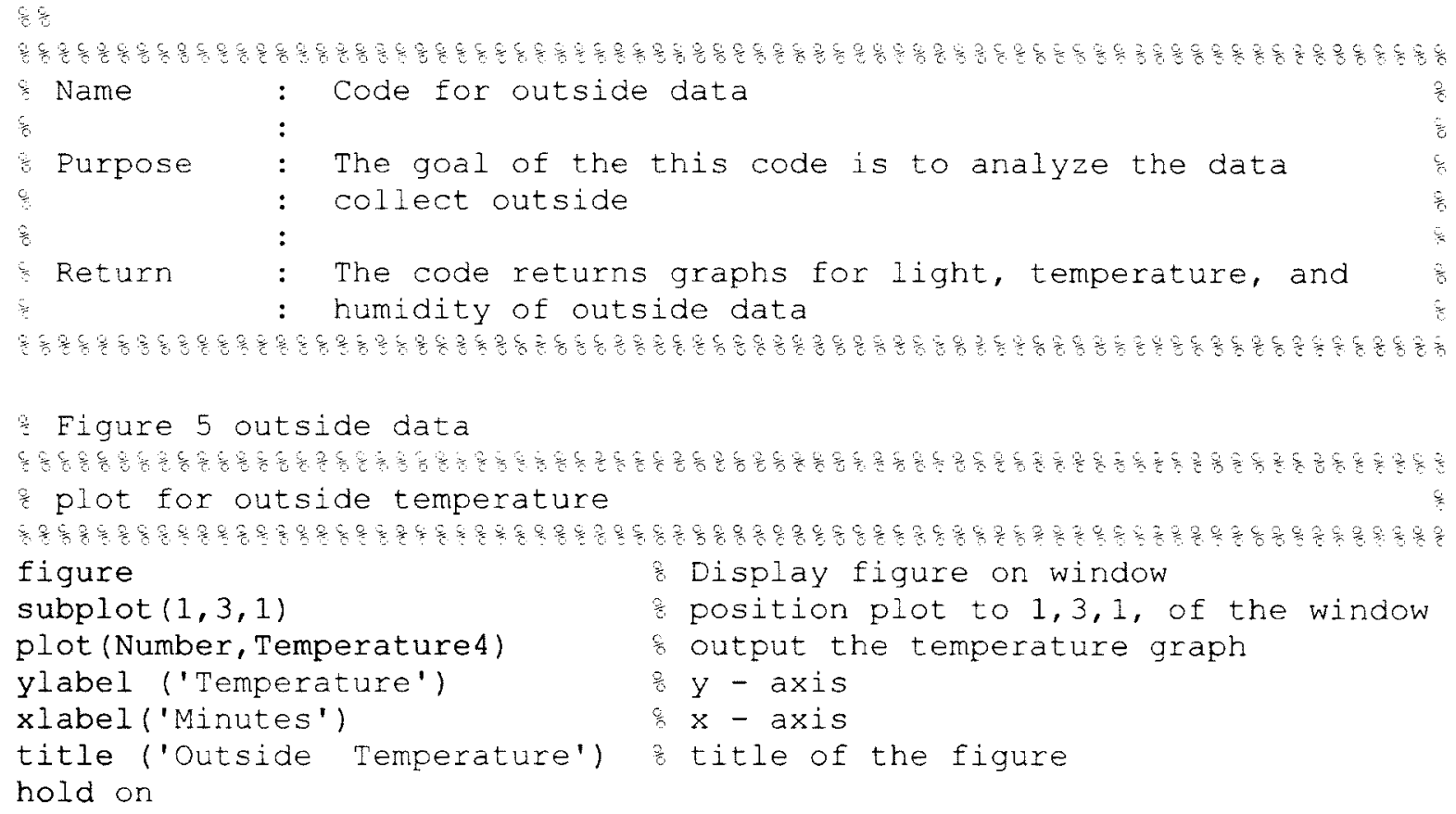




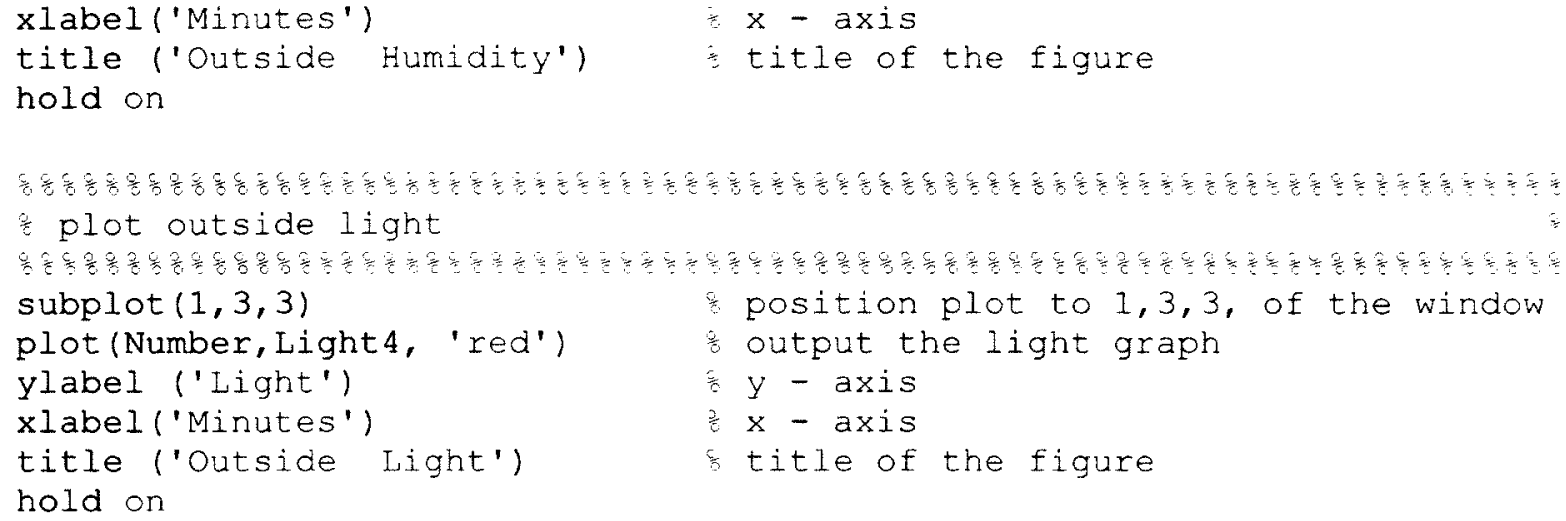




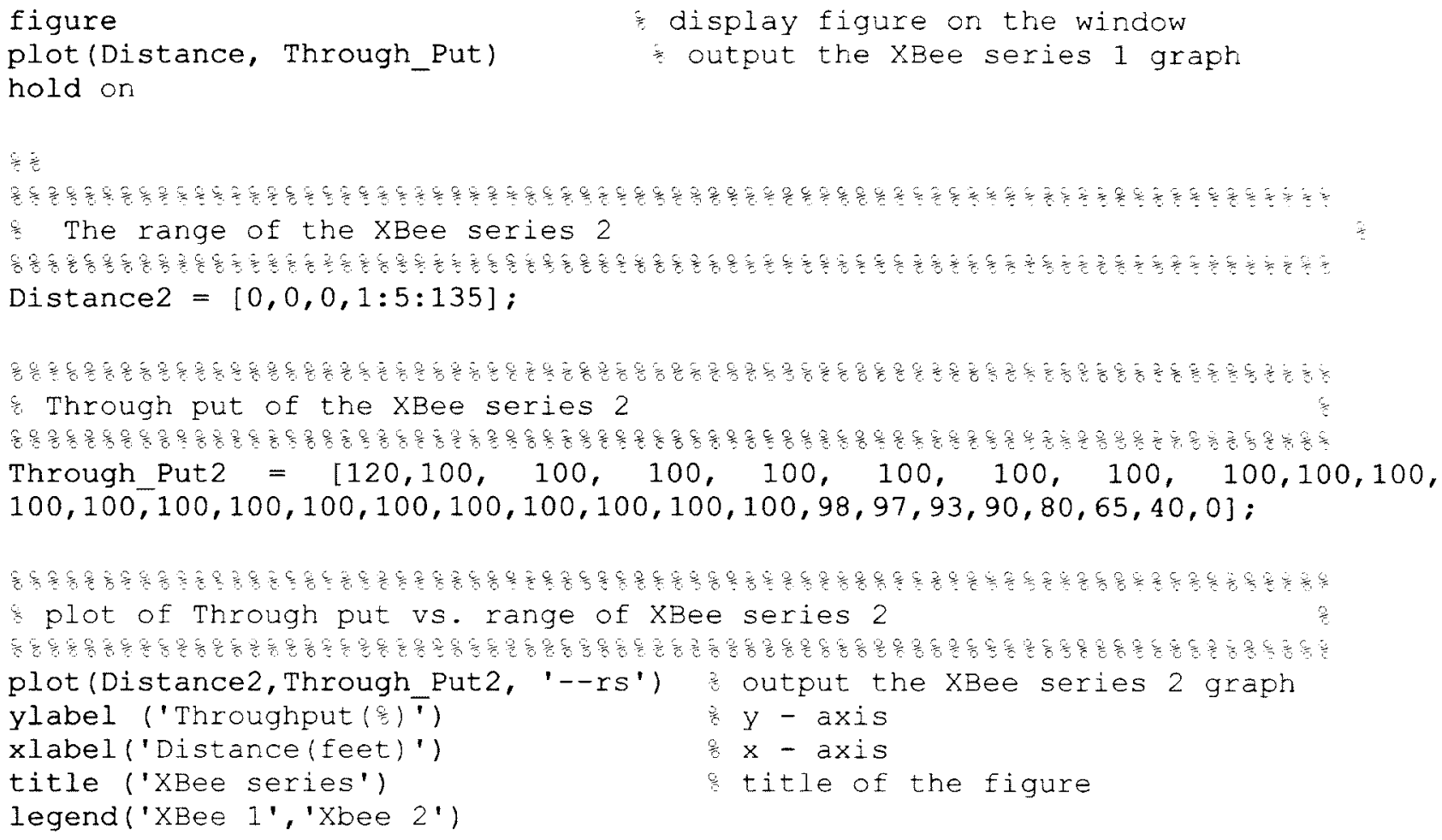




\section{Appendix I: Prototype Apartment Sample Data}

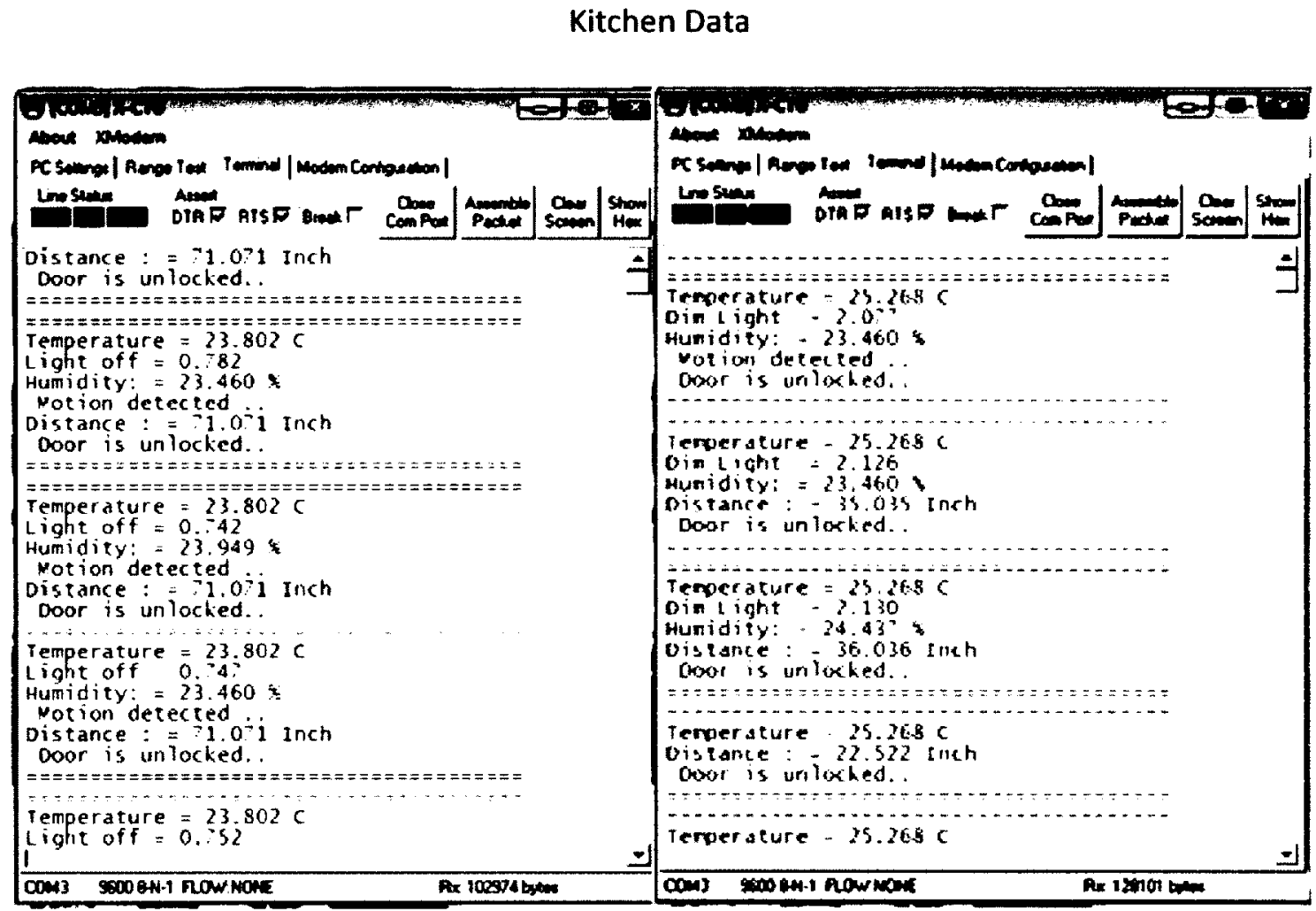

(a)

(b)

Figure 7.10: Kitchen Data Figure (a) before cooking and Figure (b) during cooking 
Living Room

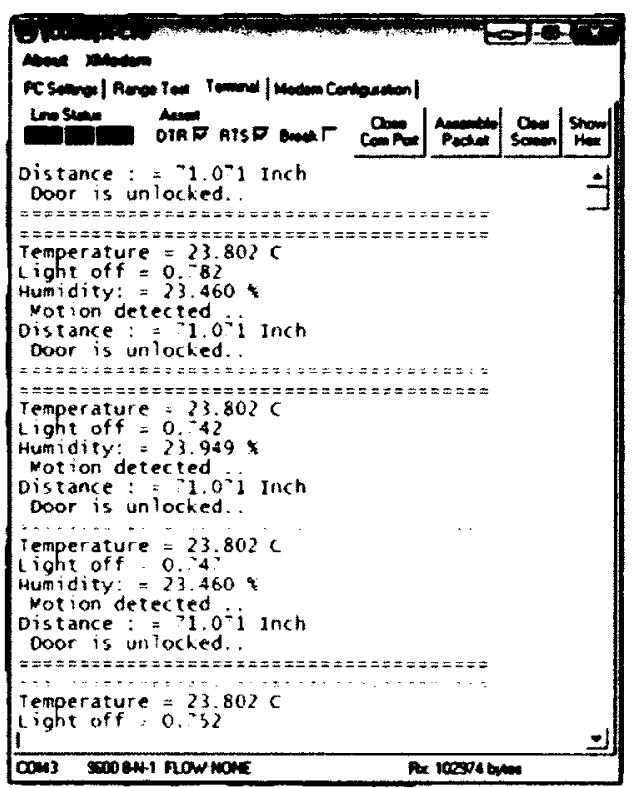

Figure 7.11: Living room Data

Toileting data

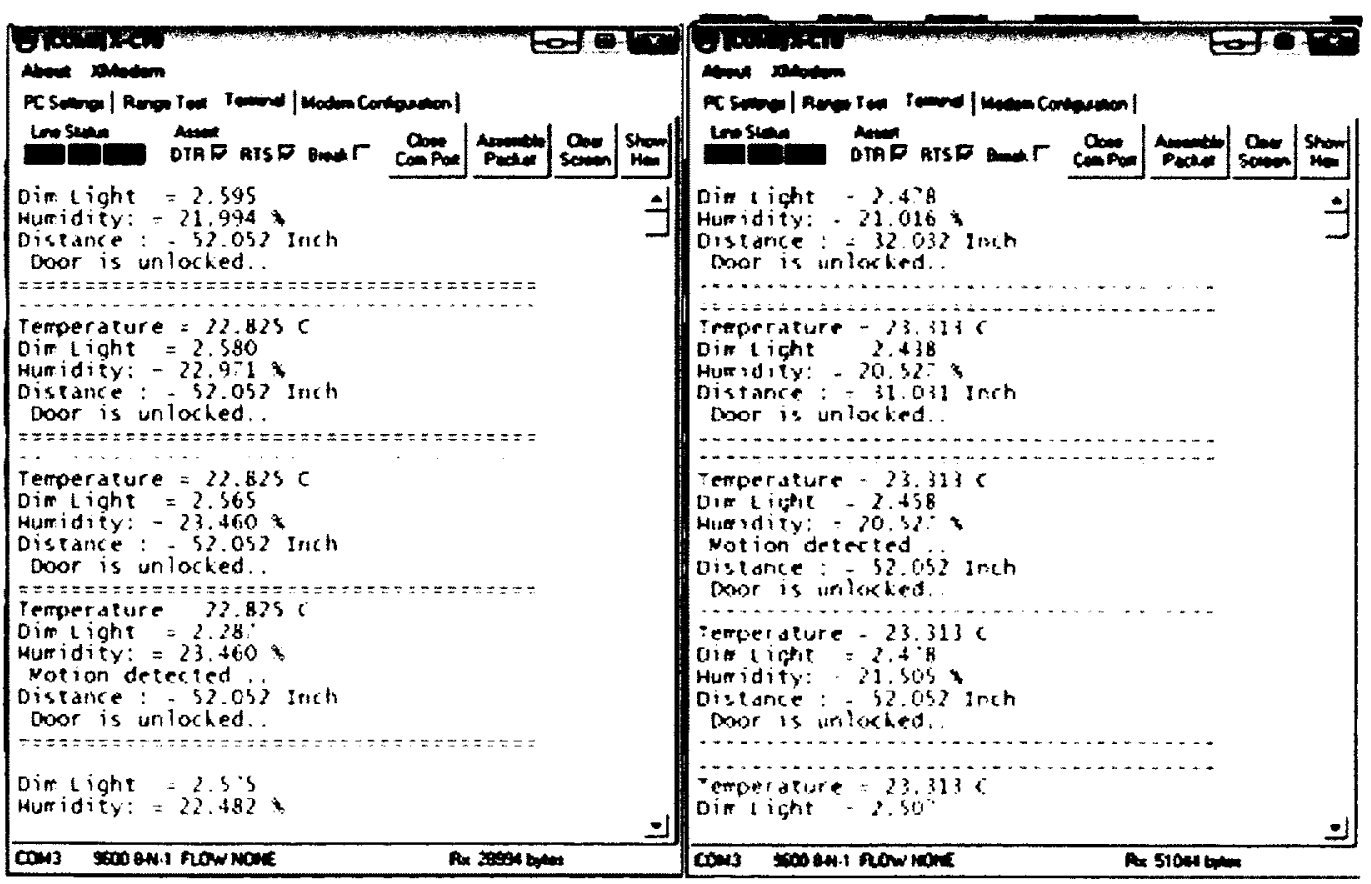

(a)

(b)

Figure 7.12: Washroom data, Figure (a) shows data before toilet and Figure (b) after toilet 


\section{Bathroom data}

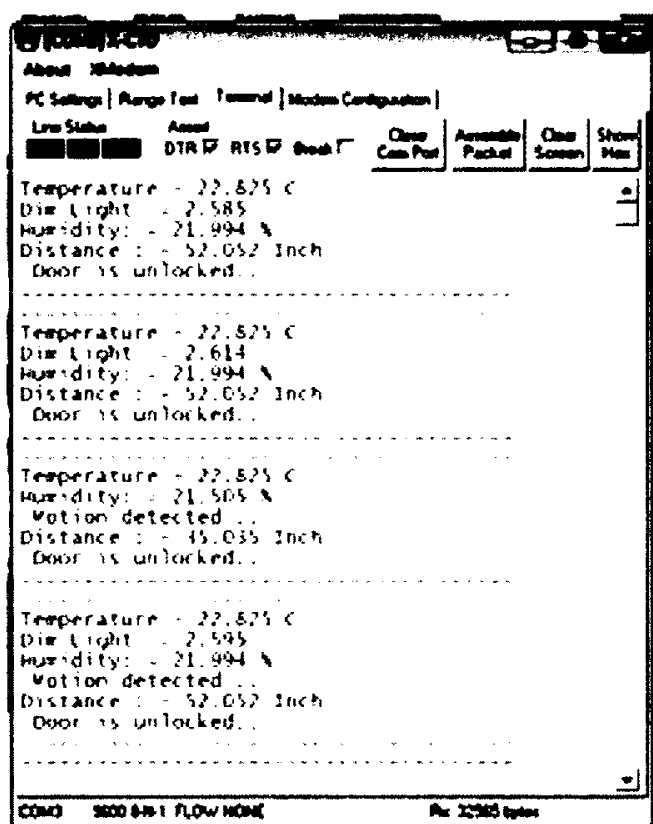

(a)

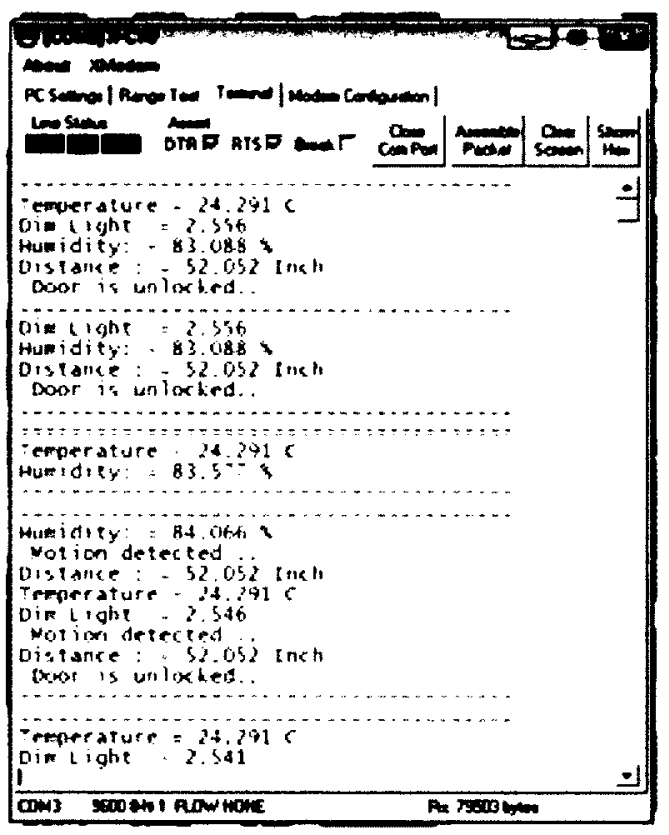

(b)

Figure 7.13: Bathroom data, Figure (a) data before shower and Figure (b) data during 